\title{
POLARIZATION-INDUCED TRANSPORT IN FERROELELCTRIC ORGANIC FIELD-EFFECT TRANSISTORS
}

\author{
A Dissertation \\ presented to \\ the Faculty of the Graduate School \\ at the University of Missouri-Columbia \\ In Partial Fulfillment \\ of the Requirements for the Degree \\ Doctor of Philosophy \\ by \\ AMRIT PRASAD LAUDARI \\ Dr. Suchi Guha, Dissertation Supervisor \\ MAY 2019
}


The undersigned, appointed by the dean of the Graduate School, have examined the dissertation entitled

\section{POLARIZATION-INDUCED TRANSPORT IN FERROELECTRIC ORGANIC FIELD- EFFECT TRANSTISTORS}

presented by Amrit Prasad Laudari,

a candidate for the degree of Doctor of Philosophy,

and hereby certify that, in their opinion, it is worthy of acceptance.

\begin{tabular}{c}
\hline Professor Suchi Guha \\
\hline Professor Shubhra Gangopadhaya \\
\hline Professor Carsten Ullrich \\
\hline
\end{tabular}

Professor Guang Bian 
This work is dedicated to its foundation; my country and my family. 


\section{Acknowledgements}

I express my sincere gratitude to my research adviser, Professor Suchi Guha. This accomplishment is possible because of her exceptional motivation, support, and guidance. Throughout the research, I found her as a shining example of what an adviser should be: supportive, helpful, patient, generous, and always available. I am indebted to her for all she has done for me. I am very grateful for her invaluable guidance to my success.

I am greatly thankful for the invaluable input from the members of my committee, Professor Shubhra Gangopadhaya, Prof. Carsten Ullrich, and Professor Guang Bian. Their excitement for research and insightful questions were tremendously helpful. I remain grateful to Prof. Giovanni Vignale, Prof. Ping Yu and Prof. Paul Miceli for their time and inputs.

My immeasurable gratitude goes to Prof. Kartik Ghosh from Missouri State University, Springfield. His instruction has given me a fantastic foundation and his mentoring and encouragement has always been far beyond what any one person deserves. I further want to thank my collaborators Prof. John E Anthony at University of Kentucky, Prof. Shadi Shahedipour-Sandvik at SUNY Polytechnic Institute, and Prof. Christopher Arendse and Dr. Theophillus Muller at University of the Western Cape, South Africa.

I appreciate the generous funding of the National Science Foundation who made this work possible. I also appreciate the wonderful support of my senior colleagues Danish and Grant, and group members Soma and Alec, who were ever-ready to assist and share useful information. 


\section{Table of Contents}

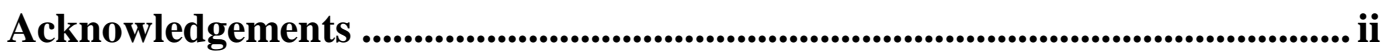

List of figures.......................................................................................................................... vii

List of tables...................................................................................................... xix

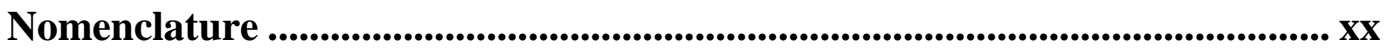

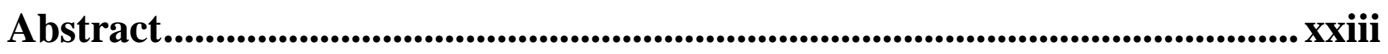

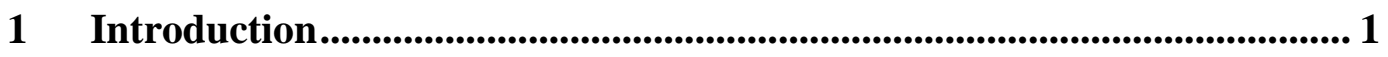

2 Organic FETs: materials, charge transport and working principle ........ 4

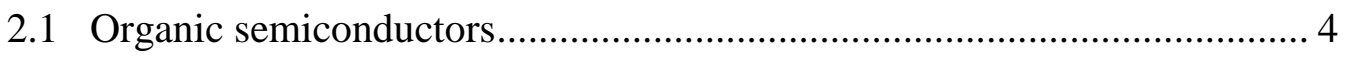

2.2 Charge transport in organic semiconductors..................................... 8

2.3 Dielectrics used in organic FETs ............................................... 11

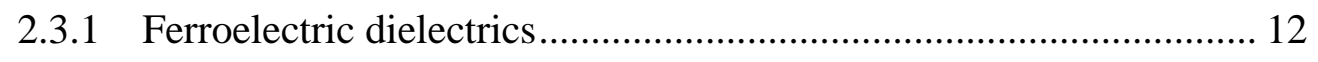

2.3.2 Non-polar (non-ferroelectric) dielectrics ................................. 20

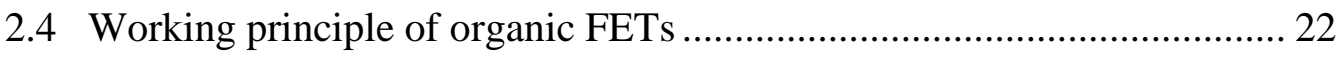

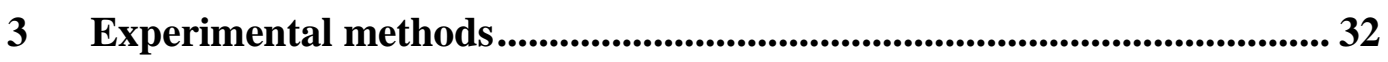

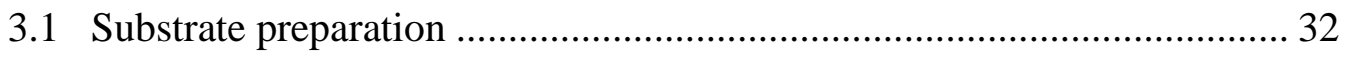

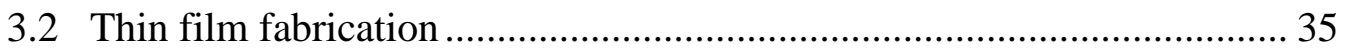




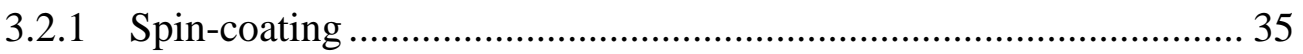

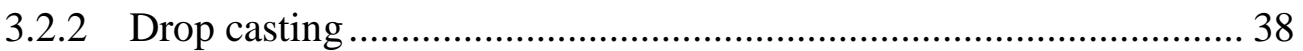

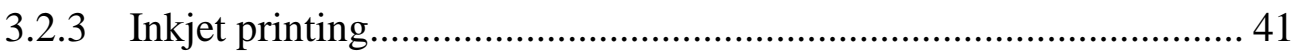

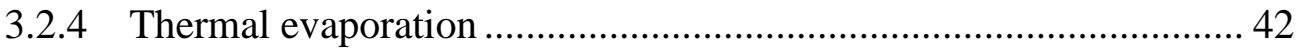

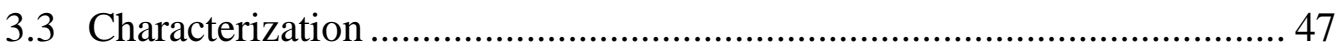

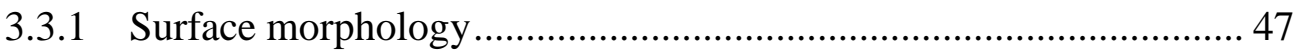

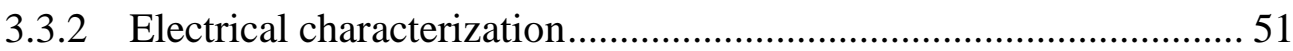

4 The role of Fröhlich polarons in pentacene FETs ........................................ 56

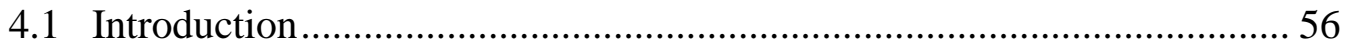

4.2 Comparison of polar versus non-polar dielectric .................................... 58

4.3 Thickness-dependence of the ferroelectric layer …………………........... 62

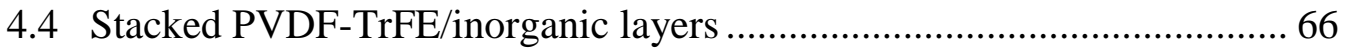

4.5 Raman scattering from pentacene on PVDF-TrFE ................................... 69

5 Transport properties in TIPS-pentacene FETs and diodes ...................... 72

5.1 Charge transport models in disordered systems........................................ 72

5.1.1 Space-charge-limited-conduction (SCLC) and shallow traps........... 76

5.2 Transport in two terminal devices............................................................ 77

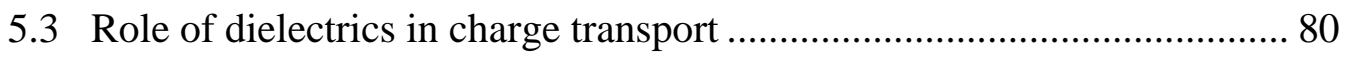

5.4 Carrier transport in TIPS-pentacene FETs ............................................... 82 
5.4.1 Temperature-dependent FET characteristics with non-ferroelectric dielectrics 84

5.4.2 Temperature-dependent FET characteristics with PVDF-TrFE ....... 85

\section{Dipole engineering of ferroelectric dielectrics- enhancement in FET}

performance.......................................................................................................................... 92

6.1 Polarization rotation in the dielectric PVDF-TrFE ................................... 93

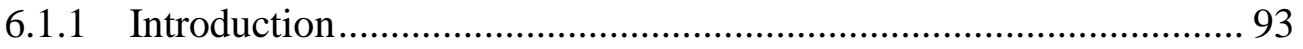

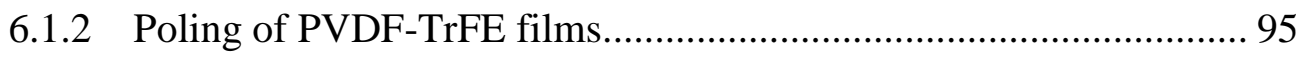

6.1.3 Small-molecule FETs with poled and unpoled PVDF-TrFE ............ 97

6.1.4 Capacitance-voltage characteristics of PVDF-TrFE metal-insulatormetal capacitors 105

6.1.5 Structure and morphology comparison at different poling conditions . 108

6.2 Textured poling of dielectric PVDF-TrFE 116

6.2.1 Technique used for textured poling of PVDF-TrFE and FET performance 116

6.2.2 Poling field optimization, microscopy and simulation 122

7 Summary and suggested future works

7.1 Summary 131

7.2 Suggested future works 133 
7.2.1 Fast switching organic FETs by dipole engineering of PVDF-TrFE ...

7.2.2 Carrier transport mechanism and FET mobility using non-linear

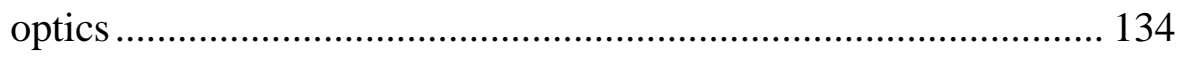

7.2.3 Low operating organic FETs for sensing applications ................ 136

References ................................................................................................................................ 138

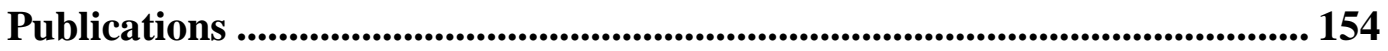

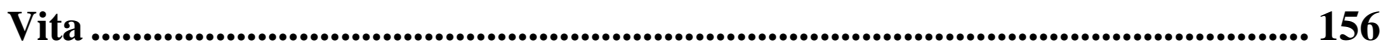




\section{List of figures}

Figure 2.1 (a) Conjugated backbone and $2 \mathrm{P}_{\mathrm{z}}$ orbitals pointing out of the molecular plane (b) highest occupied molecular orbitals and lowest unoccupied molecular orbital. The red

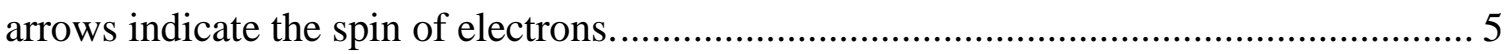

Figure 2.2 (a) Molecular structure of pentacene (b) $3 \times 3 \mathrm{~nm}^{2}$ STM images of pentacene molecules adsorbed on a $\mathrm{Ni}(111)$ substrate at RT (1.49 nA and $1.8 \mathrm{mV}$. A molecular model was also superimposed (adapted from reference [7]) .................................... 6

Figure 2.3 Molecular structure of (a) dinaphtho[2,3-b:2',3'- f]thieno[3,2-b]thiophene (DNTT), and (b)6,13-bis(triisopropylsilylethynyl)pentacene (TIPS-pentacene).

Figure 2.4 (a) Optical image of thermally evaporated pentacene on top of patterned Aluminum on a glass substrate. (b) Optical image of drop casted TIPS-pentacene on a

$\mathrm{Si}++/ \mathrm{SiO}_{2}$ substrate 8

Figure 2.5 (a) Variation of charge with voltage in polar dielectric. (b) Dipoles in polar dielectric, arrow shows the direction of polarization. (c) Variation of polarization with an

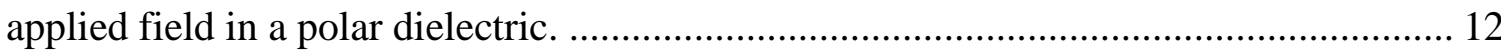

Figure 2.6 Molecular structure of PVDF-TrFE . .................................................... 13

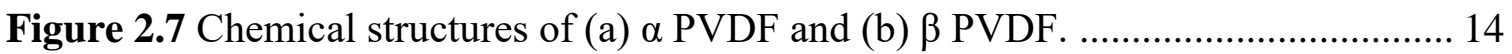

Figure 2.8 (a) Chemical structure of $\beta$ PVDF-TrFE. (b) Raman spectrum of a PVDF-

TrFE film. (c) FTIR spectrum of a PVDF-TrFE films. ............................................. 15

Figure 2.9 (a) Polarization versus voltage measurement of PVDF-TrFE film using a

Sawyer-Tower circuit. (b) SEM image of as casted thin film of PVDF-TrFE................ 15

Figure 2.10 Schematics for SHG experimental set up transmission geometry............... 17 
Figure 2.11 (a)Unpoled PVDF-TrFE, a relaxaor polymer has crystalline domains with directed dipole moments in an amorphous matrix. Application of a vertical electric field, while heating the film just above the ferroelectric-paraelectric transition temperatures, results in an alignment of the net dipole moment. (b) Experimental SHG polarimetric response of the p-polarization component from poled PVDF-TrFE film. (c) Filled circles denote the experimental p-polarization SHG data and the black dotted line is a fit to Eq.

Figure 2.12 (a) Molecular structure of PVP (b) Optical image of printed PVP (c)

Molecular structure of CYTOP

Figure 2.13 Schematics of different FET geometries used in this work. (a) bottom-gate, top-contact; (b) bottom gate, bottom-contact; (c) top-gate, bottom-contact (d) dual gate.

Figure 2.14 Energy-level diagrams across the semiconductor/dielectric interface of organic transistors and along the carrier channel. (a) Accumulation of positive charge carriers for negative gate-source voltages ( $\mathrm{p}$-channel transistor). (b) The accumulation of negative carriers for positive gate-source voltages (n-channel transistor). (c) The transport of positive carriers from the source through the semiconductor to the drain in the case of a p-channel transistor. (d) The transport of negative carriers from the source through the semiconductor to the drain in the case of an n-channel transistor.

Figure 2.15 Schematic of the operating regimes of an FET. (a) Linear regime. (b) Pinch off. (c) Saturation regime. 27

Figure 2.16 Sample (a) output and (b) transfer characteristics of an organic FET. In (a) four output curves corresponding to four gate voltages are shown. The saturation of IDS is viii 
clearly seen in each of the curves. In (b) both the $\mathrm{I}_{\mathrm{DS}}$ and the square root of $\mathrm{I}_{\mathrm{DS}}$ are plotted. IDS is plotted on a logarithm scale to better show the switching behavior of the FET. The linear behavior of the square root of $\mathrm{I}_{\mathrm{DS}}$ is also clearly seen.

Figure 3.1 (a) Schematics of drop casting method (b) drop casted thin film of TIPSpentacene

Figure3.2 (a) SEM image of spin coated TIPS-pentacene. (b) Transfer from spin coated TIPS-pentacene FET. (c) SEM image of drop cased TIPS-pentacene. (d) Transfer from drop casted TIPS-pentacene FET. Channel length of both FETs was $50 \mu \mathrm{m}$.

Figure 3.3 (a) The jetting waveform used for printing PVP. (b) Drop watcher image of ejection of PVP solution.

Figure 3.4 Picture of (a) inside thermal evaporation chamber (b) MBraun glove box with thermal evaporator in left box and spin coater in right box.

Figure 3.5 Sample mask design used for patterning top contacts for organic FETs and capacitors. (a) Gate electrode. (b) Source drain electrodes. (c) Optical images of FETs and capacitor devices.

Figure 3.6 (a) AFM images from drop casted TIPS-pentacene (b) Spin-coated PVDF$\operatorname{TrFE}$

Figure 3.7 SEM images from spin coated PVDF-TrFE on aluminum coated glass with (a) $1 \mathrm{kV}$ (b) $5 \mathrm{kV}$ accelerating voltage. 50

Figure 3.8 Three test liquid drops on the surface of printed PVP (a) water (b) formamide (c) diiodomethane. 
Figure 3.9 (a) MIS Capacitance-Voltage from Al/PVDF-TrFE/pentacene/Au structure (b) MIM Capacitance-frequency characteristics from Al/PVDF-TrFE/Al structure (c) dissipation factor from Al/PVDF-TrFE/Al structure............................................ 53

Figure 3.10 Photograph of (a) sample on the stage inside the cryostat (b) Modified closed-cycle helium cryostat (APD Cryogenics).

Figure 4.1 Schematic of a ferroelectric-based FET where the dynamic coupling of charge carriers to the electronic polarization at the semiconductor-dielectric interface is illustrated. In addition to the bulk disorder within the semiconductor, there is trapping of charges at the interface 58

Figure 4.2 (a) Transfer curves at different temperatures from $100 \mathrm{~K}$ to $350 \mathrm{~K}$ for a pentacene FET with PMMA as the dielectric layer. (b) Transfer curves at different temperatures from $200 \mathrm{~K}$ to $440 \mathrm{~K}$ for a pentacene FET with PVDF-TrFE as the dielectric layer. In both cases, $\mathrm{V}_{\mathrm{DS}}=-4 \mathrm{~V}$ 59 Figure 4.3 Variation of threshold voltage with temperature (a) PMMA FET and (b) PVDF-TrFE TET.

Figure 4.4 Comparison of the charge carrier mobility as a function of temperature in pentacene FETs using PMMA and PVDF-TrFE as dielectric layers. The dielectric constant of PVDF-TrFE as a function of temperature shown in the figure is from Ref. 42.

Figure 4.5 Arrhenius plots of FET mobilities in the ferroelectric phase for varying thicknesses of the PVDF-TrFE layer. 
Figure 4.6 (a) Comparison of the trapped charge density of the PMMA-based pentacene OFET with a PVDF-TrFE-based OFET. (b) The trapped charge density as a function of temperature for the 20nm, 90nm, and 130nm thick PVDF-TrFE devices.

Figure 4.7 Comparison of single layer PVDF-TrFE and stacked PVDF-TrFE/ SiO2

OFETs with (a) 20nm, (b) 90nm, and (c) 500nm, of the PVDF-TrFE thickness. 66

Figure 4.8 Temperature-dependent mobility of Fröhlich polarons for pentacene FETs with three thicknesses of the PVDF-TrFE layer. The bold line is a fit to the model discussed in the text. 68

Figure 4.9 Raman spectra of pentacene on PVDF-TrFE as a function of temperature. .. 70 Figure 5.1 (a) Schematic of DOS for a crystalline and an amorphous semiconductor. The mobility edges in an amorphous semiconductor play a similar role as the band edges in a crystal. (b) Schematic of trap states. (c) Schematic of discrete trap space-charge-limited conduction in a log-log plot of current density $(\mathrm{J})$ versus voltage. 73 Figure 5.2 (a) J-V characteristics (log-log plot) of pentacene at $300 \mathrm{~K}$. The inset shows the J-V behavior at different temperatures. The chemical structure of a pentacene molecule is shown in the inset. (b) Product of the trapping fraction and carrier mobility as a function of temperature for a pentacene diode. (Inset) Schematic of the energy levels. 78 Figure 5.3 (a) Chemical structure of TIPS-pentacene and an optical image from a TIPSpentacene device. (b) and (c) J-V curves from a TIPS-pentacene two-terminal device as a function of temperature. (d) J-V curve (log-log plot) at $300 \mathrm{~K}$ showing all four regions, characteristics of discrete trap SCLC. Inset shows the bulk carrier mobilities as a function of temperature. 79 
Figure 5.4 Schematic of FET structures. (a) Bottom gate PVDF-TrFE (chemical structure shown) or PVP (b) dual FET with PVDF-TrFE as bottom gate and CYTOP as top gate (c) bottom gate $\mathrm{SiO}_{2}$ (d) Average carrier mobility of TIPS-pentacene for different gate dielectrics. 81

Figure 5.5 (a)-(c) Transconductance curves as a function of temperature from TIPSpentacene FETs with CYTOP, $\mathrm{SiO}_{2}$ and $\mathrm{Al}_{2} \mathrm{O}_{3}$ dielectrics. (d) $\mu_{\mathrm{FET}}$ mobilities as a function of temperature for $\mathrm{SiO}_{2}, \mathrm{Al}_{2} \mathrm{O}_{3}, \mathrm{PVP}$ and CYTOP dielectrics. (e) The maximum trap density obtained from subthreshold swing values in CYTOP and $\mathrm{Al}_{2} \mathrm{O}_{3}$ based TIPSpentacene FETs. 85

Figure 5.6 (a) Transconductance curves as a function of temperature from TIPSpentacene FET with PVDF-TrFE as the dielectric layer. The inset magnifies the transfer curves upwards of $160 \mathrm{~K}$. (b) The carrier mobilities for PVDF-TrFE based TIPSpentacene FET extracted from transconductance curves swept at $V_{D S}=-10 \mathrm{~V}$ and $-20 \mathrm{~V}$. The dashed oval highlights the decreasing trend in $\mu$ beyond $240 \mathrm{~K}$. 86

Figure 5.7 (a) The carrier mobility of TIPS-pentacene FET with CYTOP as the dielectric (left axis) and PVDF-TrFE dielectric (right axis) for the dual geometry device shown in the inset. (b) Transfer sweeps at $200 \mathrm{~K}$ and $300 \mathrm{~K}$ for TIPS-pentacene FET (with PVDFTrFE) showing both drain and the leakage current. (c) The carrier mobility of TIPSpentacene FET with PVDF-TrFE and SiO2/PVDF-TrFE bilayer as the dielectric.

Figure 5.8 Fits to the experimental TIPS-pentance $\mu$ data using unpoled PVDF-TrFE as the dielectric. The temperature range between 100- $220 \mathrm{~K}$ is fit with Eq. 5.4. The high temperature range for the sweep at $\mathrm{V}_{\mathrm{DS}}$ at $-20 \mathrm{~V}$ has been fit with $\mu \propto \mathrm{T}^{-\mathrm{n}}$ 89 
Figure 5.9 (a) TIPS-pentacene FET mobility as a function of temperature at two different VDS values for unpoled PVDF-TrFE. The blue triangles are the FET mobility from a TIPS-pentacene FET where the PVDF-TrFE dielectric layer was vertically poled. (b)The trapped charge density as a function of temperature for TIPS-pentacene FETs using unpoled and vertically poled PVDF-TrFE.

Figure 6.1 Poling of PVDF-TrFE films. (a) Schematic of vertical and (b) Lateral poling of PVDF-TrFE, respectively. Arrows denote the polarization direction in the crystalline phases of the ferroelectric slab after poling. 96 Figure 6.2 Output and Transfer characteristics of DNTT-based FETs. (a-c) Output characteristics of DNTT FET with unpoled, V-poled, and L-poled PVDF-TrFE (d) Transfer characteristics of DNTT FETs with V-poled, unpoled, and L-poled PVDF-TrFE. The black line depicts a linear fit to the subthreshold region. (e) Transfer characteristics of a vertically poled FET by applying various magnitudes of a lateral voltage. Green and blue curves depict devices where the lateral field is applied such that the polarization direction of the dielectric is oriented parallel to the external source-drain electric field. (f) Transfer curves of the same FET device shown in (e) after reversing the polarity of the lateral field, such that the polarization direction is oriented antiparallel to the source-drain electric field. 97

Figure 6.3 (a) Transfer characteristics of pentacene FETs with V-poled, unpoled, and Lpoled PVDF-TrFE (b) Transfer curve from V-poled TIPS-pentacene based FET. All curves were swept in the saturation region regime where the drain-source voltage $\left(V_{D S}\right)$ was maintained at $-2 \mathrm{~V}$, and the gate voltage $\left(\mathrm{V}_{\mathrm{GS}}\right)$ was varied from 0 to $-2 \mathrm{~V}$. 100 
Figure 6.4 Typical capacitance versus voltage characteristics of (a) DNTT MIS (b) pentacene MIS devices.

Figure 6.5 Interface potential and total hole density. (a) The interface potential versus $U_{g}$, the gate voltage above the flat band voltage. The black curve is for a V-poled PVDFTrFE DNTT FET. The red and blue curves are after L-poling the dielectric layer by applying a $-50 \mathrm{~V}$ and $-100 \mathrm{~V}$ to the dielectric as explained in the text. (b) The hole density as a function of the interface potential for a V-poled and L-poling (by applying $50 \mathrm{~V}$ ) of the V-poled DNTT FET. 103

Figure 6.6 Schematic transfer curves of PVDF-TrFE-based FETs for different poling conditions. Vth may be positive or negative. A slightly positive Vth for the L-poled FET results in a higher current at zero gate bias condition depicted by the circle). The dipole orientation in PVDF-TrFE with poling conditions are also shown. 104

Figure 6.7 Capacitance - voltage characteristics of PVDF-TrFE MIM capacitors. (a) Capacitance versus voltage curves for V-poled, L-poled, and unpoled PVDF-TrFE films. The arrows show the direction of the applied voltage. (b) Corresponding tan $\delta$ values from the three films. (c) Capacitance versus voltage curves after L-poling of a V-poled film.

Figure 6.8 Typical FET characteristics of DNTT based devices with $30 \mathrm{~nm}$ PVDF-TrFE layer. (a) The voltage between source and drain was swept from 0 to $-1.6 \mathrm{~V}$ to obtain the output characteristics at various gate voltages varying from 0 to $-1.6 \mathrm{~V}$. (b) The transfer curve was swept in the saturation region where the drain-source voltage $\left(\mathrm{V}_{\mathrm{DS}}\right)$ was maintained at $-1.6 \mathrm{~V}$, and the gate voltage $\left(\mathrm{V}_{\mathrm{GS}}\right)$ was varied from 0 to $-1.6 \mathrm{~V}$. Both output and transfer curves were from a V-poled PVDF-TrFE based FET. (c) Transfer 
characteristics of a V-poled FET after applying two different lateral electric fields. The lateral field was applied such that the polarization direction of the dielectric is oriented parallel to the external source-drain electric field 107

Figure 6.9 Structure and morphology of PVDF-TrFE as a function of poling. (a)-(c) Scanning electron micrographs from unpoled, V-poled, and laterally L-poled films. (d) Grazing-incidence XRD from unpoled, V-poled, and Lpoled PVDF-TrFE films on Si substrate. The (hkl) indexing is based on the ferroelectric phase as discussed in the text. (e) The XRD from the V-poled film and after L-poling the same V-poled film. Deconvolution of two peaks at $2 \theta(\mathrm{deg})(\beta$ phase $)$ and at $18^{\circ}$ for the paraelectric phase (PE) are shown for the laterally poled film. 110

Figure 6.10 DFT optimization of a PVDF-TrFE molecule in a solvent atmosphere. The charge distribution with no external field (top) and in the presence of an external field applied perpendicular to the chain axis (bottom). One unit of PVDF-TrFE (3:1) molecule was optimized in the presence of DMF solvent with no external field. The optimized molecule was again optimized with the applied electric field.

Figure 6.11 (a) DFT calculations of the structural changes on a PVDF-TrFE oligomer enclosed in a dielectric medium upon the application of an external electric field. Blue arrows indicate the direction of the dipole moment. (b-d) Structural changes of PVDFTrFE after the application of a lateral electric field with various magnitude and directions. The application of a lateral field buckles the molecule (representative of a combination of the $\alpha$ and $\delta$ phase), shown in the middle 114

Figure 6.12 Textured poling in PVDF-TrFE films for application in TIPS-pentacene FETs. (a) Schematic representation of the FET geometry with three electrodes at the 
bottom. The two lateral Al strips serve as electrodes for applying the lateral voltage after the PVDF-TrFE film was vertically poled. Schematics of vertical and textured poling are represented. (b) Optical image of a bottom-gate top-contact TIPS pentacene FET. (c) Transfer characteristics from a vertically poled PVDF-TrFE/TIPS-pentacene device. The gate leakage current is shown in red. (d) Transfer characteristics from a textured poled PVDF-TrFE/TIPS-pentacene device. The gate leakage current is shown in red 117 Figure 6.13 Typical transfer and square root of current plots to show how the saturation mobility and subthreshold swing (SS) parameters were extracted. (a) Transfer characteristics and square root of IDS of a vertically poled PVDF-TrFE/TIPS-pentacene FET. (b) Transfer characteristics and square root of IDS of a textured poled PVDFTrFE/TIPS-pentacene FET. 119

Figure 6.14 TIPS-pentacene FET characteristics for V poled and textured poled PVDFTrFE films. (a) Output characteristics of a TIPS-pentacene FET with V poled PVDFTrFE (black squares). The same device was then L poled from the bottom for various magnitudes of electric field; output characteristics of the same FET for two such L poling conditions. (b) Transfer characteristics from the V poled PVDF-TrFE/TIPS-pentacene device and the same device after $\mathrm{L}$ poling from the bottom (with a field of $9 \times 10^{4} \mathrm{~V} / \mathrm{m}$ ). Both IDS and the square root of IDS as a function of the gate voltage are shown. The drainsource voltage was held at $-4 \mathrm{~V}$. (c) The product of the total resistance and the channel width (W) versus channel length (L) for three different values of W/L for V poled (black square) and textured poled (red circle) TIPS-pentacene FETs. (d) Optical image of a substrate containing the TIPS-pentacene FETs along with the lateral electrodes that are placed below the PVDF-TrFE layer. 
Figure 6.15 Transfer characteristics of TIPS-pentacene FETs with different poling conditions of the PVDF-TrFE layer and surface morphology. (a) Transfer characteristics of a V-poled PVDF-TrFE layer (black) and the transfer curve for the same device after Lpoling (from the bottom) using an electric field of $10^{5} \mathrm{~V} / \mathrm{m}$ (red). (b) Transfer curve of a V-poled FET (black) and after L-poling the same device from the top with an electric field of $6 \times 10^{3} \mathrm{~V} / \mathrm{m}$ (red). (c) SEM image of a V-poled PVDF-TrFE film. (d) AFM image of a V poled PVDF-TrFE film. (e) AFM image of a V-poled PVDF-TrFE film, which

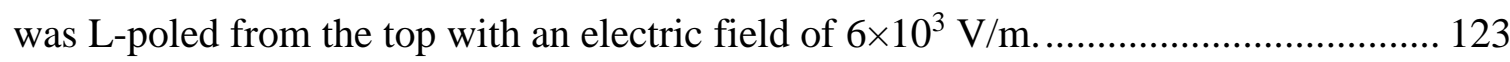
Figure 6.16 Cross-sectional TEM images of poled and unpoled PVDF-TrFE layer. (a) and (b) Cross-sectional TEM images from a textured poled PVDF-TrFE film. The PVDFTrFE films of thickness $~ 300 \mathrm{~nm}$ was deposited on Al coated glass. The image in (a) shows a slightly different shading near Al due to lateral poling. In (b), this region near the electrode is seen to grow due to additional polarization during the measurements. (c) and (d) Cross-sectional TEM images from an unpoled PVDF-TrFE film. The PVDF-TrFE films looks smooth and no different shading is seen near the Al electrode at the onset in (c). Due to additional polarization, a different shading occurs for $\sim 20 \mathrm{~nm}$ of the film near the $\mathrm{Al}$ electrode after a few measurements 126

Figure 6.17 Meshing scheme for the simulated device stack and field distribution in insulating slab. (a) A refinement of $100 \mathrm{~nm} \times 100 \mathrm{~nm}$ was made surrounding the dielectric and source/drain Al contacts. (b)The central Al electrode had meshing of $10 \mathrm{~nm}$ x $10 \mathrm{~nm}$. The global mesh (glass substrate below, and air above) was set to $10 \mu \mathrm{m}$ by 10 $\mu \mathrm{m}$. (c) The schematics of the device setup for the simulations. (d)The plot shows the simulated electric field distribution within the dielectric layer. The blue curve shows the Xvii 
field distribution as seen within the dielectric slab. The inset shows the region of interest, namely, the field distribution in the dielectric layer just above the middle (gate) electrode.

Figure 7.1 (a) FET design as a sensor and (b) a photograph of such device based on

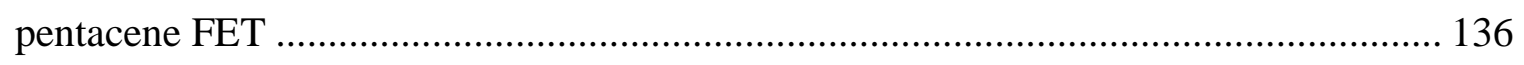




\section{List of tables}

Table 2-I Dielectric constant of dielectrics used in this research. ............................... 22

Table 3-I Recipes used for spin coating of major polymers used in this work............... 37

Table 4-I The polaron hopping barrier and hopping lengths obtained by fits to Eq. (4.5).

Table 6-I The FET parameters extracted from unpoled V-poled, and L-poled PVDF-

TrFE from DNTT and pentacene FETs.

Table 6-II FET parameters for a vertically poled PVDF-TrFE film using DNTT as the organic semiconductor. Lateral electric field is applied in the direction of $\mathbf{E}_{\mathrm{DS}}$, and then the polarity is reversed. 102

Table 6-III Dipole moment of a PVDF-TrFE (in a solvent bath) upon application of an external electric field in the lateral direction. 112

Table 6-IV Dipole moment of a PVDF-TrFE (in a solvent bath) upon application of an external electric field in the perpendicular direction. 113 


\section{Nomenclature}

FET - field-effect transistor

MIS - metal-insulator-semiconductor

MIM - metal-insulator-metal

AFM - atomic force microscope

SEM - scanning electron microscope

XRD - x-ray diffraction

C-V - capacitance-voltage

G-V - conductance-voltage

C-f - capacitance-frequency

I-V - current-voltage

$\mathrm{V}_{\mathrm{GS}}$ - gate voltage

$\mathrm{V}_{\mathrm{DS}}$ - drain-source voltage

$\mathrm{V}_{\mathrm{Th}}$ - threshold voltage

$\Delta \mathrm{V}_{\mathrm{Th}}$ - threshold voltage shift

$\mathrm{I}_{\mathrm{DS}}{ }^{-}$drain-source current 


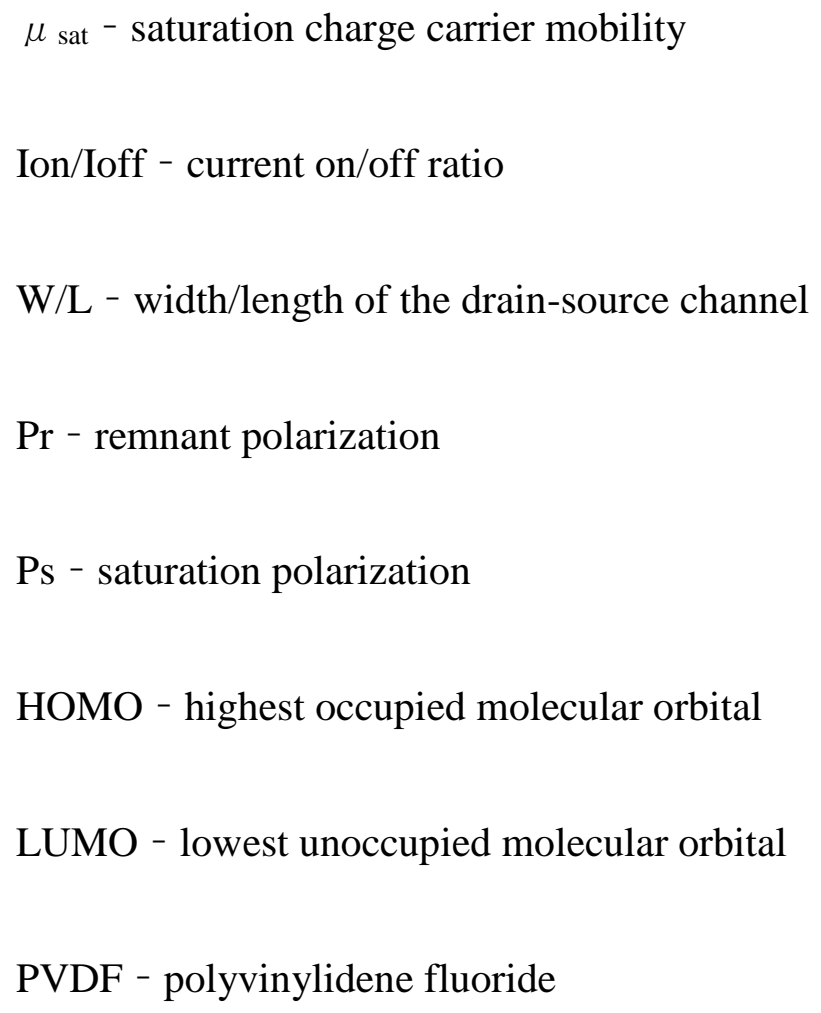

PVDF-TrFE - polyvinylidene fluoride-trifluoroethylene

PVP - poly-4-vinyl phenol

PGMEA - propylene glycol monomethyl ether acetate

PMMA - polymethyl methacrylate

$\mathrm{SiO}_{2}$ - silicon dioxide

DNTT - dinaphtho[2,3-b:2',3'-f]thieno[3,2-b]thiophene

TIPS-pentacene - 6,13-bis(triisopropylsilylethynyl)pentacene

DMSO - dimethyl sulfoxide 
DMF - dimethyl formamide

wt\% - weight percent (measure of solution concentration)

CCD - charge-coupled device

TRM-SHG - time-resolved microscopic optical second harmonic generation

EFISHG - Electric field-induced second-harmonic generation 


\begin{abstract}
In this research we study the role of ferroelectric dielectrics in organic field-effect transistors (FETs) to understand the mechanism of charge transport in organic semiconductors. The ferroelectric nature of the polymer, poly(vinylidene fluoride) (PVDF)), has been known for over 45 years. However, its role in interfacial transport in organic/polymeric FETs is not that well understood. PVDF and its copolymer, polyvinylidene fluoride-trifluoroethylene (PVDF-TrFE), as a dielectric in organic FETs is a perfect test-bed for conducting transport studies where a systematic tuning of the dielectric constant with temperature may be achieved. By choosing small molecule organic semiconductors - pentacene and 6,13 bis(triisopropylsilylethynyl)pentacene (TIPSpentacene) - along with a copolymer PVDF-TrFE as the dielectric layer, the FET characteristics are monitored as a function of temperature. Pentacene FETs show a weak temperature dependence of the charge carrier mobility in the ferroelectric phase of PVDFTrFE, which is attributed to polarization fluctuation driven transport resulting from a coupling of the charge carriers to the surface phonons of the dielectric layer. A negative coefficient of carrier mobility is observed in TIPS-pentacene upwards of $200 \mathrm{~K}$ with the ferroelectric dielectric, while an activated transport is observed with non-ferroelectric dielectrics. We show that this behavior is correlated with the nature of the trap states in TIPS-pentacene.
\end{abstract}

We also developed the method of dipole engineering of the PVDF-TrFE films to enhance the properties of organic FETs. PVDF-TrFE, despite its applications in a vast range of work (including as a gate dielectric in organic FET and sensing applications) poses 
concerns such as higher conductivity compared to other polymer non-ferroelectric dielectrics. We have come up with new methods of electrical poling the dielectric layer to enhance FET performance as well as reduce gate leakage issues. We demonstrate the effect of polarization rotation in PVDF-TrFE on the performance of small-molecule-based organic FETs. The subthreshold swing and other transistor parameters in organic FETs can be controlled in a reversible fashion by switching the polarization direction in the PVDFTrFE layer. We further demonstrate a novel method of selective poling of the dielectric layer. By using solution processed TIPS-pentacene as the organic semiconductor, it is shown that textured poling of the PVDF-TrFE layer dramatically improves FET properties compared to unpoled or uniformly poled ferroelectric films. The texturing is achieved by first vertically poling the PVDF-TrFE film and then laterally poling the dielectric layer close to the gate electrode. TIPS-pentacene FETs show on/off ratios of $10^{5}$ and hole mobilities of $1 \mathrm{~cm}^{2} / \mathrm{Vs}$ under ambient conditions with operating voltages well below $-4 \mathrm{~V}$. This research opens prospects of achieving low-operating FETs without any expensive patterning techniques.

Keywords: Organic semiconductor, organic field-effect transistor, polymer ferroelectric, charge transport, band-like transport, Fröhlich polaron, polarization rotation, poling 


\section{Introduction}

Organic electronics is a field of electronics which utilizes conducting polymers and molecules. The field of organic electronics has grown significantly after the discovery of the first highly conductive polymer - polyacetylene -in 1977, by A. J. Heeger, H. Shirakawa, and Alan G. MacDiamid (Nobel Prize in Chemistry in 2000). ${ }^{1}$ One of the most significant milestones in organic semiconductors was the realization of organic field effect transistors (FETs) by a polythiophene transistor by Tsumora et al. in $1986 .^{2}$ The building blocks of organic semiconductors are mainly carbon and hydrogen atoms. Some orbitals hybridize (forming covalent bonds between carbon atoms) while other orbitals remain unhybridized. ${ }^{3}$ Overlapping of adjacent unhybridized orbitals results in shared molecular orbitals and this is responsible for carrier transport and semiconducting properties in these materials. Some examples of organic electronic devices are organic FETs, light emitting diodes (LEDs), solar cells and photodetectors.

The research activities in organic devices are flourishing due to their promise in large-area electronics fabricated on flexible substrates using low-cost and unconventional means such as spin coating, room temperature printing, and roll-to-roll processing making them attractive for an ever-growing range of technological applications. Interestingly, some organic FETs have shown performances better than amorphous silicon thin film transistors and are already utilized in the market of displays such as in OLED TVs by LG.Inc. While much work has been done in the field of organic semiconductors, the field is still new compared to the research and understanding of traditional electronics based on inorganic semiconductors. Thus, further work is required before realizing the full potential 
of organic electronics and their applications. Many fundamental aspects of charge carrier transport in an organic semiconductor are not fully understood yet.

In this research, we fabricate and characterize organic FETs using several organic semiconductors and polymer dielectrics. We also use conventional oxide-based dielectrics for a comparative study. One main objective of this research is to study the role of polar dielectrics in organic FETs to understand the mechanism of charge transport in organic semiconductors. The second objective of this research is to develop dipole engineering techniques in polar dielectrics to enhance the FET properties.

In chapter 2 , we begin with a brief introduction of organic semiconductors and polymer dielectrics including ferroelectric co-polymer poly(vinylidene fluoride-cotrifluoroethylene) (PVDF-TrFE), the main dielectric used in this work. This chapter also contains a brief description of the mechanism of ferroelectricity in PVDF-TrFE and some results for its phase conformation which has been employed in this report. Chapter 2 also introduces organic FETs and the geometry of organic FETs used in this work. Chapter 3 explores the experimental methods employed in this work which contains three sections; substrate preparation, device fabrication, and characterization. The device fabrication part gives an overview of thin film deposition techniques for organic devices whereas the characterization section discusses concise descriptions of employed device characterization techniques. Chapter 4 marks the beginning of experimental results. In that chapter we discuss the role of Fröhlich polarons in pentacene based organic FETs. It includes a detailed temperature dependent electrical characterization of organic FETs with various thicknesses of PVDF-TrFE dielectric and stacked dielectric layers to decouple the 
role of Fröhlich polarons from the short-range Holstein polarons. Chapter 5 is a discussion on the general mechanism of transport involving traps using solution-processed organic semiconductor, 6,13 bis(triisopropylsilylethynyl)pentacene (TIPS-pentacene). This chapter focuses on the role of traps FETs and bulk transport. Here, a comparative study of non-ferroelectric based organic FETs with PVDFTrFE based organic FETs is presented to demonstrate the role of the ferroelectric dielectric in de-trapping of the shallow-trap states into more mobile states in TIPS-pentacene. Chapter 6 discusses new techniques we have developed to enhance the properties of organic FETs using an external electric field to modify the ferroelectric layer. The first part of this chapter explains the effect of polarization rotation by $90^{\circ}$ in ferroelectric organic FETs, the second part presents the results of textured poling of the ferroelectric dielectric layer resulting in a dramatic improvement of organic FETs. This report ends with concluding remarks and suggestions for future work in chapter 7. 


\section{Organic FETs: materials, charge transport and working principle}

\subsection{Organic semiconductors}

Organic semiconductors are typically carbon and hydrogen-based materials that can transport charges i.e., conduct an electrical current through them. A common structural property of all organic semiconductors is the alternation of single and double bonds between carbon atoms called "conjugation". Covalent bonds between carbon atoms of such a backbone are formed by three $\mathrm{sp}^{2}$ hybridized orbitals, meaning the $2 \mathrm{~s}, 2 \mathrm{p}_{\mathrm{x}}$ and $2 \mathrm{p}_{\mathrm{y}}$ orbitals combine to form three new orbitals, while the $2 p_{z}$ orbital remains unhybridized. ${ }^{3}$ One can picture a molecular plane, as shown in figure 2.1 ; unhybridized $2 \mathrm{p}_{z}$ orbitals provide electron clouds above and below the plane. The adjacent $2 p_{z}$ orbitals overlap each other. This overlapping results in shared molecular orbitals which are often referred as an extended $\pi$-system. Electrons in these orbitals belong to the whole $\pi$-system, but not to specific carbon atoms, resulting in delocalization. A $\pi$-system can be extended over a conjugated segment or even over the entire organic molecule. The in-phase combination of the $2 p_{z}$ orbitals results in electrons from neighboring atoms lying in the highest occupied molecular orbital (HOMO) and is labeled as the $\pi$ orbital. The next highest molecular orbital is empty and is called the lowest unoccupied molecular orbital (LUMO). It is formed by the out-of-phase combination of the $2 \mathrm{p}_{\mathrm{z}}$ orbitals and is labeled the $\pi^{*}$ orbital. In the ground state, following the Pauli Exclusion Principle, two electrons of opposite spins per each orbital fill orbitals of the lowest energies. The HOMO and the LUMO are important for electrical conductivity and optical properties of organic semiconductors. The 
energy difference between the HOMO and LUMO level is crucial from a device perspective, and typically has values between 1.5 and $3.5 \mathrm{eV}$ for organic semiconductors. The typical HOMO-LUMO gap of semiconductors we used in this research is about $2 \mathrm{eV}$.

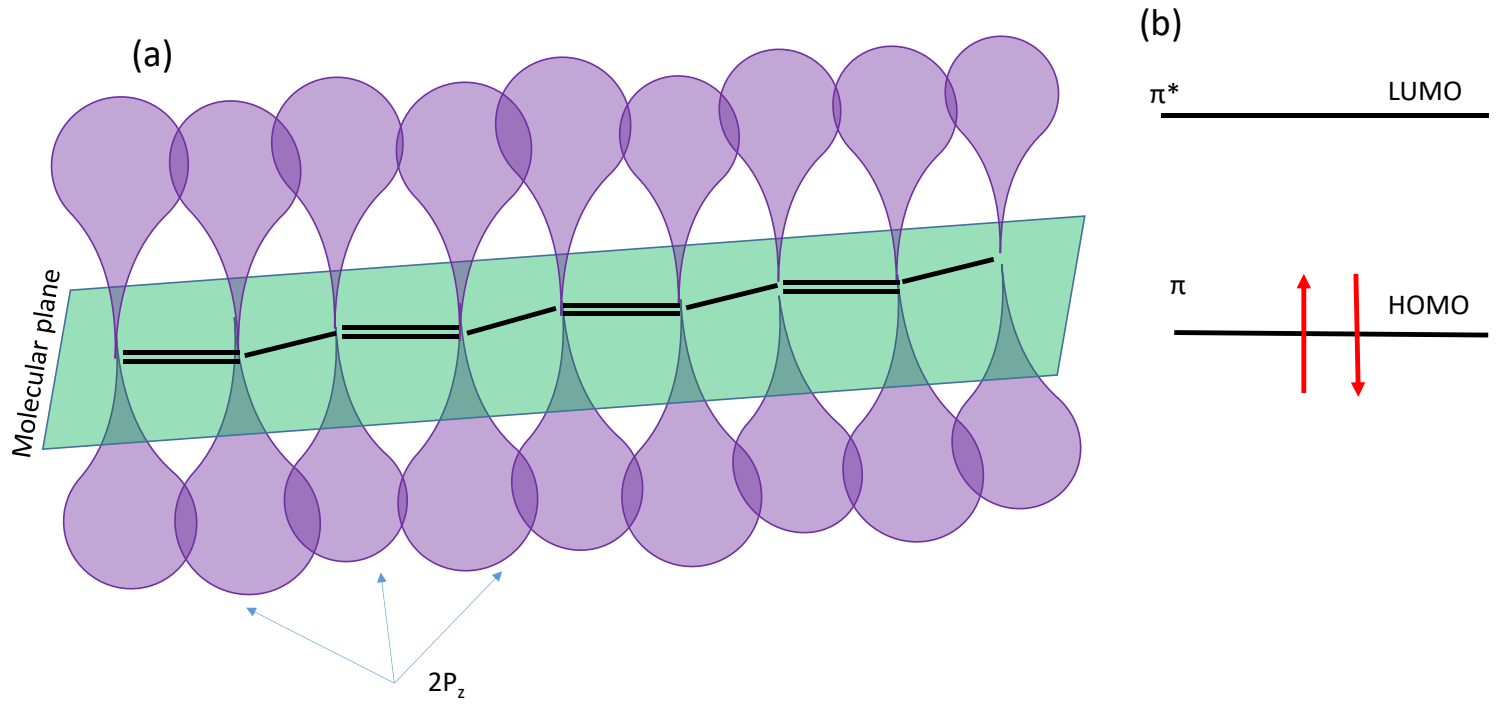

Figure 2.1 (a) Conjugated backbone and $2 \mathrm{P}_{\mathrm{z}}$ orbitals pointing out of the molecular plane (b) highest occupied molecular orbitals and lowest unoccupied molecular orbital. The red arrows indicate the spin of electrons.

In this work we use small molecule organic semiconductors: pentacene, dinaphtho[2,3-b:2',3'- $\quad$ f]thieno[3,2-b]thiophene $\quad($ DNTT), $\quad$ and $\quad 6,13-$ bis(triisopropylsilylethynyl)pentacene (TIPS-pentacene). Pentacene is an aromatic hydrocarbon comprised of five fused benzene rings and has been extensively studied because of its ability to provide comparatively high charge carrier mobility for an organic semiconductor. ${ }^{4}, 5$ Pentacene forms polycrystalline films in a herringbone packing structure. Thermally evaporated pentacene films form single crystal islands on the surface of the dielectric, generally dendritic in shape. ${ }^{6}$ Like most of the small molecule semiconductors, it is generally deposited using thermal evaporation. Figure 2.2 (a) shows 
the molecular structure of pentacene and figure 2.2 (b) shows an AFM images along with a molecular model superimposed. This image was adapted from reference. ${ }^{7}$

(a)

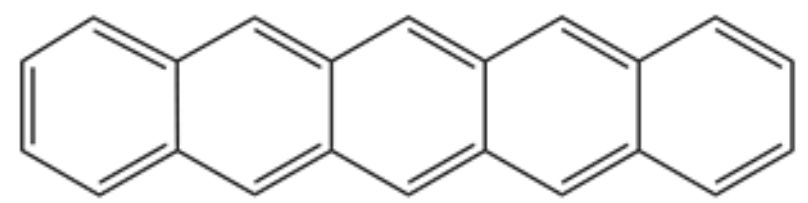

(b)

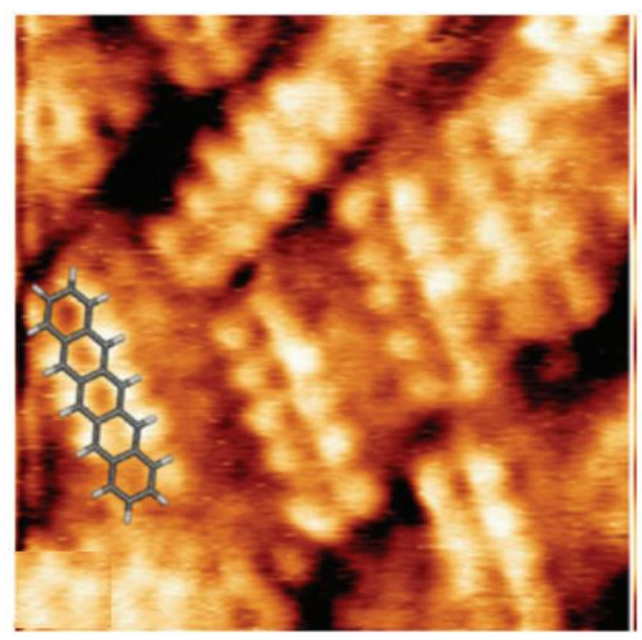

Figure 2.2 (a) Molecular structure of pentacene (b) $3 \times 3 \mathrm{~nm}^{2}$ STM images of pentacene molecules adsorbed on a $\mathrm{Ni}(111)$ substrate at $\mathrm{RT}(1.49 \mathrm{nA}$ and $1.8 \mathrm{mV}$. A molecular model was also superimposed (adapted from reference [7])

DNTT, another small molecule semiconductor, has been used in FET devices for more than a decade. ${ }^{8}$ DNTT is a highly $\pi$-extended heteroarene with six fused aromatic rings. It has a relatively low-lying HOMO level and a large HOMO-LUMO gap. It forms good FET devices that are stable in air and have high charge carrier mobility close to 2 $\mathrm{cm}^{2} / \mathrm{Vs}$ with the right choice of the dielectric layer. ${ }^{9}$ Its structure is shown in figure 2.3(a).

TIPS-pentacene is a derivative of pentacene in which two triisopropylsilylethynyl acetylene groups have been added to the center benzene ring on pentacene as shown in figure 2.3 (b). This addition of the isopropyl groups in TIPS-pentacene allows pentacene to be dissolved in many organic solvents while also increasing the carrier mobility in FETs. ${ }^{10}$ This increase in mobility in TIPS-pentacene comes from the steric hindrance 
introduced by the long sidechains. The packing of TIPS-pentacene molecules is restricted resulting in high $\pi-\pi$ overlap. The increase in $\pi-\pi$ overlap allows charges to be easily transferred between TIPS-pentacene molecules. Among the solution processable small molecule organic semiconductors, TIPS-pentacene has received a lot of attention in FETs. ${ }^{10-12}$

(a)

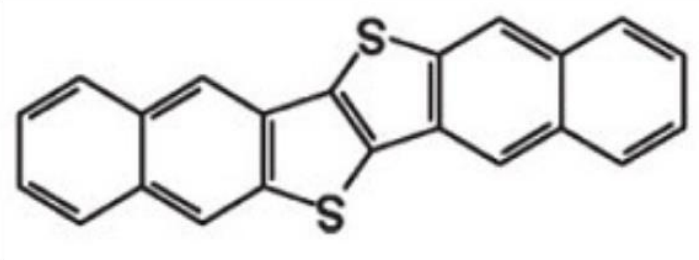

(b)

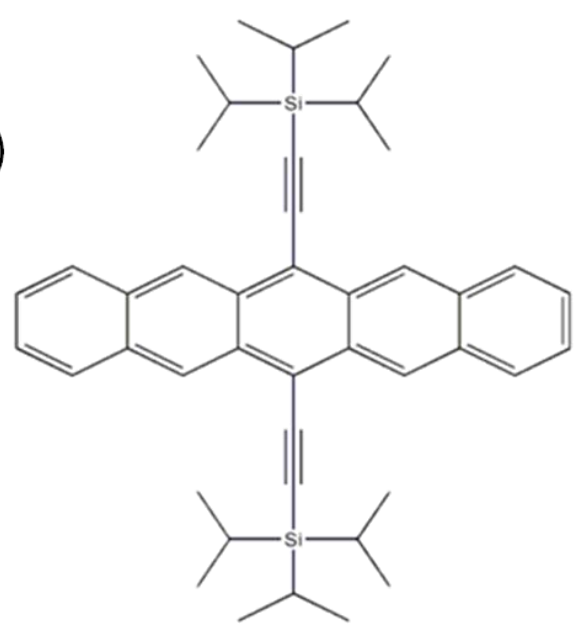

Figure 2.3 Molecular structure of (a) dinaphtho[2,3-b:2',3'- f]thieno[3,2-b]thiophene (DNTT), and (b)6,13bis(triisopropylsilylethynyl)pentacene (TIPS-pentacene).

Also, the presence of grain boundaries is greatly reduced in solution processable thin films of TIPS-pentacene as compared to thermally evaporated small molecule pentacene. Increasing the solubility of TIPS-pentacene allows for much simpler device fabrication, as spin coating or drop casting can now be employed rather than thermal evaporation, which requires equipment that is vastly more complex. This low-cost, facile fabrication is one of the most attractive aspects of the current organic FET technology today. Figure 2.4 (a) is an optical image of thermally evaporated pentacene on top of patterned aluminum on a glass substrate, and figure 2.4 (b) is optical image of drop casted TIPS-pentacene on a $\mathrm{Si}^{++} / \mathrm{SiO}_{2}$ substrate. Using the solvent trap method, as discussed in 
section 3.2.2 in detail, we get TIPS-pentacene crystals with few $\mathrm{cm}$ in length and several microns in width.
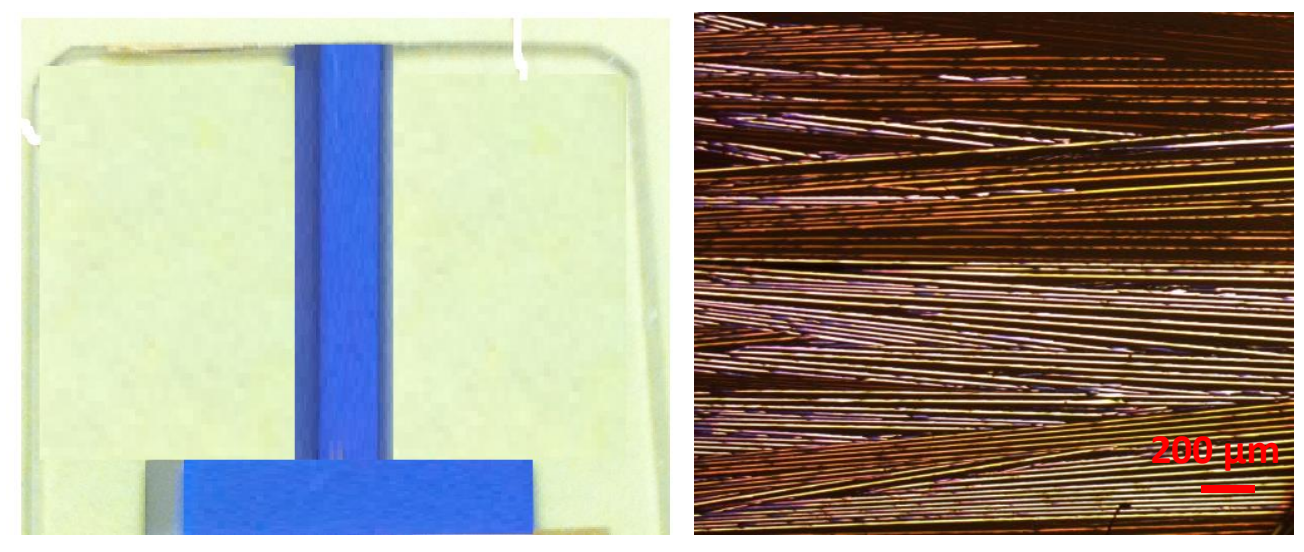

Figure 2.4 (a) Optical image of thermally evaporated pentacene on top of patterned Aluminum on a glass substrate. (b) Optical image of drop casted TIPS-pentacene on a $\mathrm{Si+}+/ \mathrm{SiO}_{2}$ substrate.

\subsection{Charge transport in organic semiconductors}

The exact nature of charge transport in organic semiconductors and devices is still open for discussion. However, tremendous efforts have been carried out in this field and have clearly shown a distinction in charge transport in disordered organic semiconductors such as amorphous polymer and highly ordered single crystal semiconductors. As discussed in section 2.1, the fundamental property that allows organic semiconductors to conduct electronic charge is molecular conjugation which causes the delocalization of one of the four valence electrons of each carbon atom. In organic solids the intermolecular bonds are due to relatively weak van der Waals interactions; the electronic wave functions usually do not extend over the entire volume of the organic solid. These wave functions are either localized to a finite number of molecules, or even to an individual molecule. The transport of charge carriers travelling through the organic semiconductor is therefore 
determined by the ease with which electrons are transported from one molecule to the next under the influence of an applied electric field. Since the carriers are not transported in traditional electronic bands, carrier mobility in organic semiconductors is expected to be thermally activated. Thermally activated charge transport, in general, is much smaller than the charge transport in silicon based and other inorganic semiconductor crystals. ${ }^{13-15}$

The transport of charges in organic molecules rely on a thermally assisted hopping mechanism via a Gaussian distribution of electronic states (discussed in detail in chapter 5). Charge transport in organic semiconductors, therefore, depends on the packing of semiconductor polymer chains or small molecules and the density of structural defects. Band to band transfer is much faster due to the available energy sites all having the same energy, while hopping transport requires charge carriers to change levels to move across the material. The fastest hopping transport occurs when there is a very tight distribution of available energy states and defects in the semiconductor are minimized. Because no single transport model can account for this wide range of observed carrier mobilities, several models have been proposed to describe charge transport in organic materials: variable range hopping ${ }^{16}$ delocalized charge transport, ${ }^{17}$ multiple trapping and release, ${ }^{18}$ and other hybrid models. ${ }^{19}$ The model of variable-range hopping (VRH) assumes that charge carriers hop between localized electronic states by quantum-mechanical tunneling through energy barriers and that the probability of a hopping event is determined by the hopping distance along with the energy distribution of the localized states. Specifically, carriers can hop over short distances or over long distances depending upon large activation energies or small activation energies, respectively. The variable-range hopping model is usually discussed in the context of amorphous semiconductor films with room-temperature 
mobilities below about $10^{-2} \mathrm{~cm}^{2} /$ Vs. Many small-molecule organic semiconductors have, however, a strong tendency to form polycrystalline films. Because of the regular molecular arrangement, the delocalized orbitals of neighboring molecules partially overlap, thereby facilitating efficient intermolecular charge-carrier transfer and carrier mobilities that are much larger than in amorphous films, usually well above $10^{-2} \mathrm{~cm}^{2} /$ Vs. Such large mobilities are not easily explained with the variable-range hopping model.

In contrast to the variable-range hopping model, the multiple trapping and release (MTR) model adapted for organic transistors by Gilles Horowitz ${ }^{20}$ and co-workers assumes that most of the charge carriers in the channel are trapped in localized states (that are associated with structural or chemical defects), and that carriers cannot move directly from one state to another. Instead, carriers are temporarily promoted to an extended-state band in which charge transport occurs.

Furthermore, temperature-dependent time-of-flight mobility measurements on highly purified pentacene crystals have shown clear evidence for charge transport in extended states with a hole mobility that is within an order of magnitude of the hole mobility in single-crystalline silicon and is limited by phonon scattering, rather than thermal activation. ${ }^{21}$

Charge transport in organic semiconductors also depend upon the device they are used for. Our research is focused on charge transport in organic thin film transistors. The dimensions of organic transistors usually far exceed the dimensions of an individual molecule. Therefore, organic transistors typically utilize a thin film in which many 
conjugated molecules are arranged in an ordered fashion. Charge transport in organic thin film transistors and diodes is discussed in more detail in chapter 5.

\subsection{Dielectrics used in organic FETs}

Dielectrics can transfer electric effects without conducting electricity and can be polar or non-polar. Polar dielectrics are those in which the molecules have a permanent dipole moment as their centers of the positive and negative charges do not coincide. The molecules are asymmetric in shape. In the case of non-polar dielectrics, the centers of both positive as well as negative charges coincide. The dipole moment of each molecule in a non-polar system is thus zero. Dielectrics can also be categorized as ferroelectric or nonferroelectric. The name "ferroelectricity" comes from an analogy to ferromagnetism (the presence of a permanent magnetic moment), which was discovered prior to ferroelectricity. Ferroelectricity is a phenomenon occurring in certain materials that arises from separation of charges. ${ }^{22}$ It refers to a spontaneous electric polarization that can be manipulated upon the application of an electric field. Dielectrics are capacitors that accumulate charges upon application of an electric field. In non-polar dielectrics, charge accumulation varies linearly with applied field and saturates at some field. Upon reversing the field, the curve follows the same path back. Figure 2.5 (a) shows variation of charge accumulation with voltage in non-polar dielectric. In polar dielectrics, polarization curve does not follow back the curve once it achieves saturation $\left(\mathrm{P}_{\mathrm{s}}\right)$. There will be some remnant polarization $\left(\mathrm{P}_{\mathrm{r}}\right)$ even after the removal of an external field. The remnant polarization signifies that a high number of dipoles remain aligned and can be flipped only when a minimum electric field required to reorient the dipoles, defined as the coercive field, of the opposite polarity is applied. Figure 
2.5 (b) shows dipoles in a polar dielectric where arrows indicate the direction of polarization, and figure 2.5 (c) illustrates the variation of polarization with an applied field in a polar dielectric.

(a)

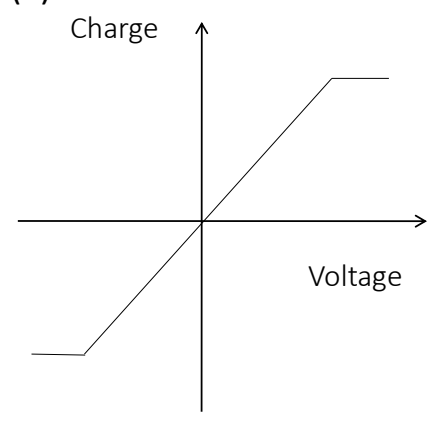

(b)

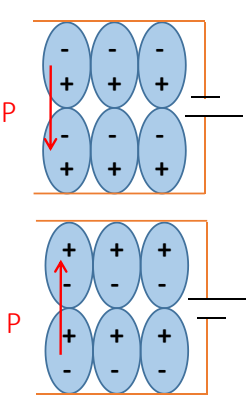

(c)

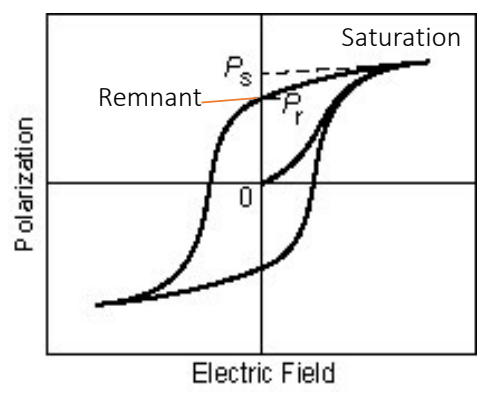

Figure 2.5 (a) Variation of charge with voltage in polar dielectric. (b) Dipoles in polar dielectric, arrow shows the direction of polarization. (c) Variation of polarization with an applied field in a polar dielectric.

\subsubsection{Ferroelectric dielectrics}

Ferroelectrics have the property of possessing macroscopic spontaneous polarization that can be reoriented through the application of an external electric field. Crystallinity is key to materials being ferroelectric. The ferroelectric phase of materials can be changed with the transition temperature called the Curie temperature (polar ferroelectrics phase to nonpolar paraelectric phase). Polymer ferroelectrics are very much governed through their semicrystallinity. The organic polymer polyvinylidene fluoride (PVDF) and its copolymers are ferroelectric materials widely used by researchers and inventors. The morphology of PVDF consists of crystalline components (with dipolar domains) in an amorphous matrix. PVDF and its copolymers are relaxor ferroelectrics, which are materials that exhibit high electrostriction. PVDF-based polymers organize into crystalline lamellae with folded chains interwoven within amorphous layers. It is important 
that the packing of PVDF chains is such that the dipoles point in the same direction. Figure 2.6 shows the molecular structure of the copolymer PVDF-TrFE. This is the ferroelectric dielectric used in our research.

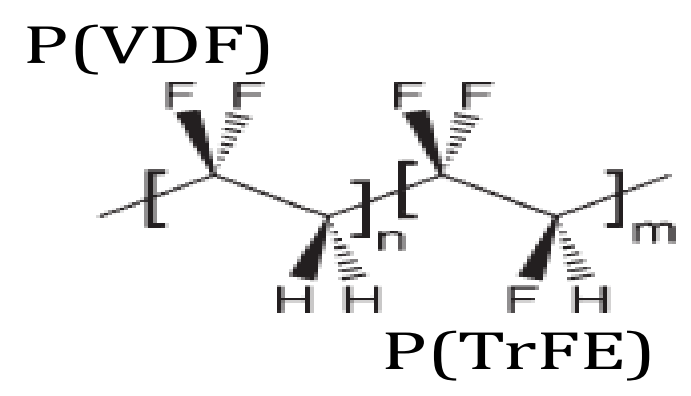

Figure 2.6 Molecular structure of PVDF-TrFE .

PVDF exists at least in four different phases: $\alpha, \beta, \gamma$, and $\delta$, of which the $\alpha$ and $\beta$ phases are the most prominent. ${ }^{23}$ The $\alpha$ phase is the paraelectric phase with a conformation of trans-gauche $\left(\mathrm{TG}^{+} \mathrm{TG}^{-}\right)$and $\beta$ PVDF is the all trans configuration (TTTT) in which the bonds on each successive carbon are $180^{\circ}$ away from the previous ones, as shown in figure 2.7 (a-b). The arrows denote the dipole moment. The $\beta$ phase of PVDF-TrFE is ferroelectric and also has nonlinear optical properties. The ferroelectric properties of $\beta$-PVDF come from the differing electronegativity of hydrogen and fluorine, which gives rise to a dipole moment for each molecule oriented perpendicular to the polymer chain. PVDF films are not ferroelectric upon spin casting; they require additional measures such as stretching of the polymer or controlled heating of the film to ensure the all-trans configuration. 
(a)

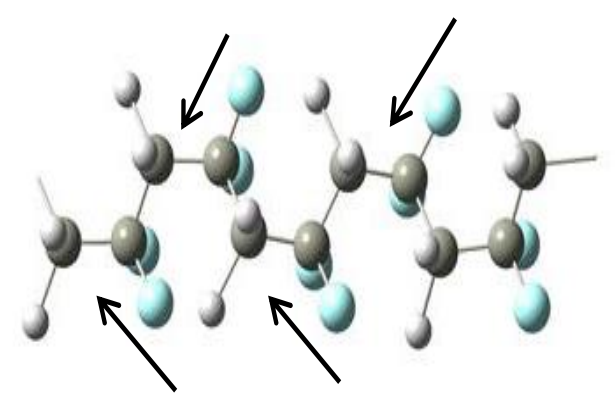

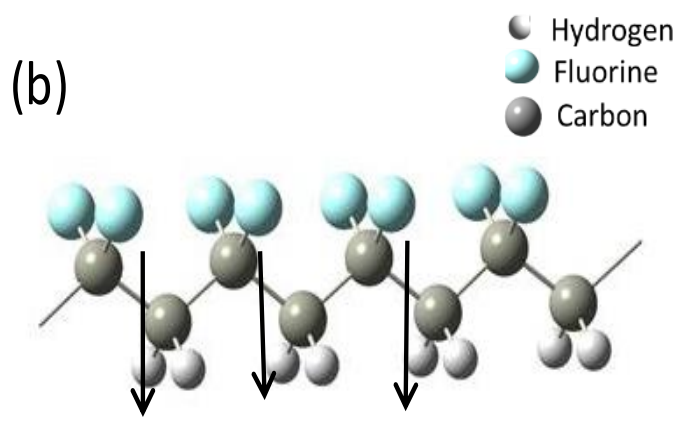

Figure 2.7 Chemical structures of (a) $\alpha$ PVDF and (b) $\beta$ PVDF.

The additional fluorine atom in TrFE monomer, as shown in figure 2.8 (a), prevents the polymer from forming the trans-gauche $\alpha$ phase as long as the molecule is above $11 \%$ TrFE. $^{24}$ The advantage of the copolymer, PVDF-TrFE, is that it is ferroelectric after directly processing the film. ${ }^{25,} 26$ It also shows a piezoelectric effect which has been investigated for more than 40 years. ${ }^{27}$

Annealing PVDF-TrFE films at $135^{\circ} \mathrm{C}$ further enhances the $\beta$ phase of the copolymer, as evident from Raman scattering and FTIR spectra [figure 2.8(b-c)]. The 840 $\mathrm{cm}^{-1}$ vibrational peak is assigned to the $\mathrm{CF}_{2}$ group in the all-trans conformation. The Raman band at $1285 \mathrm{~cm}^{-1}$ is indicative of the coupling of the $\mathrm{CF}_{2}$ stretching and skeletal CC stretching modes, a signature of the all-trans phase. The $1400 \mathrm{~cm}^{-1}$ band in the FTIR spectrum is assigned to the $\mathrm{CF}_{2}$ bending modes in all-trans segment. 
(a)

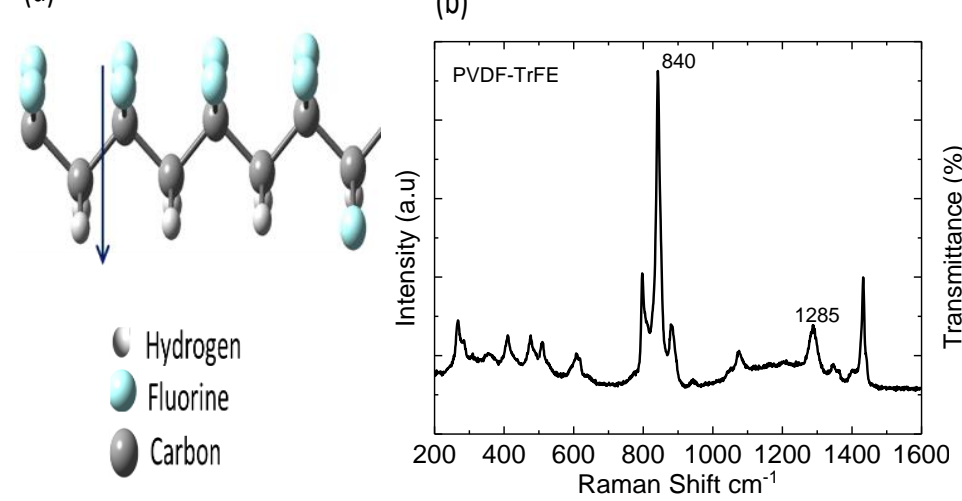

(b)

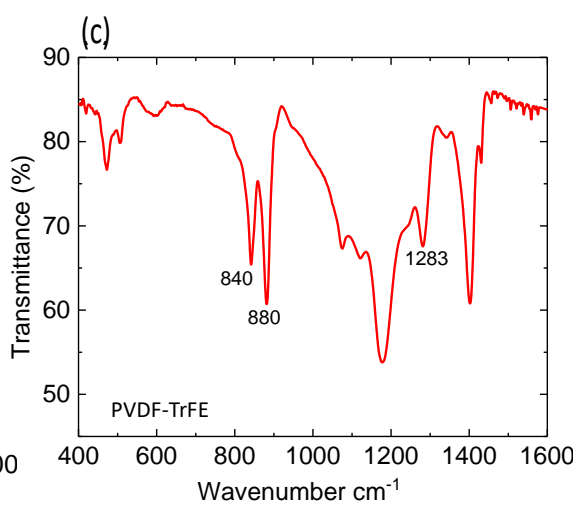

Figure 2.8 (a) Chemical structure of $\beta$ PVDF-TrFE. (b) Raman spectrum of a PVDF-TrFE film. (c) FTIR spectrum of a PVDF-TrFE films.

The ferroelectric nature of a PVDF-TrFE film can be seen in the polarization data (figure 2.9) from a metal/PVDF-TrFE/metal capacitor. These measurements were performed at $1 \mathrm{kHz}$ using a Sawyer-Tower circuit and the details can be found in reference ${ }^{28}$ The polarization versus voltage $(\mathrm{P}-\mathrm{V})$ loops are characterized by the saturation polarization. The sample remains polarized even after removal of the field. The SEM image of a PVDF-TrFE layer is shown figure 2.9 (b). PVDF-TrFE forms elongated crystalline structures in the hundreds of nm length scale.
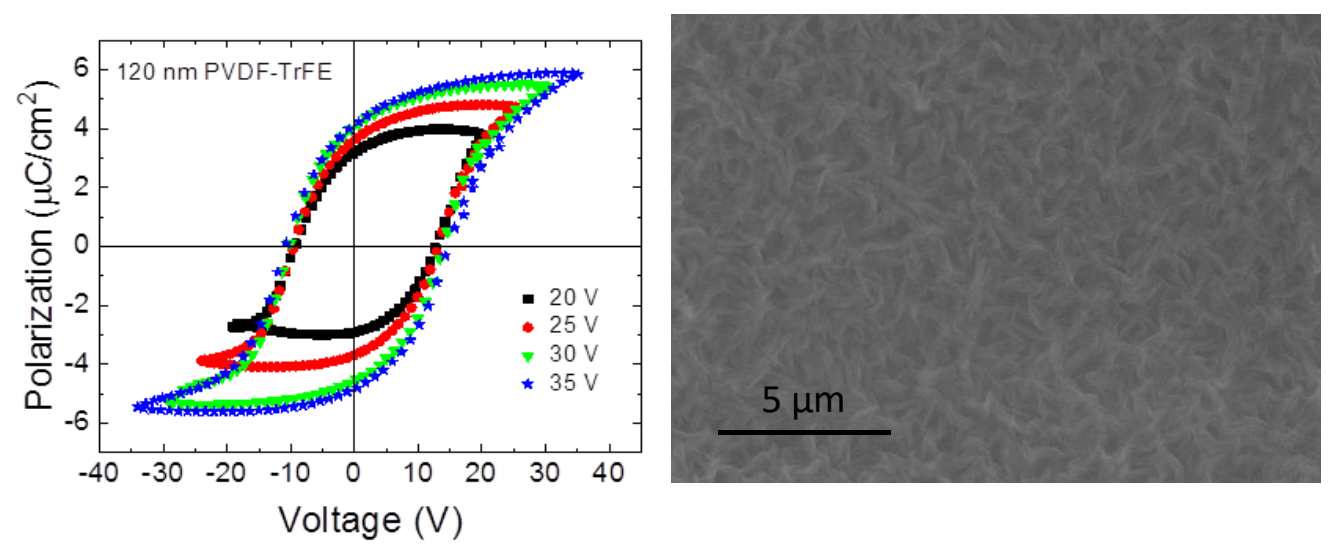

Figure 2.9 (a) Polarization versus voltage measurement of PVDF-TrFE film using a Sawyer-Tower circuit.

(b) SEM image of as casted thin film of PVDF-TrFE. 


\subsubsection{Second harmonic generation}

The $\beta$ phase of PVDF and its copolymers lacks inversion symmetry and thus results in second harmonic generation (SHG). SHG constitutes the process of doubling the frequency of the incident light. The SHG interaction of the dominant electric-dipoles involves three photons; two "input" photons of frequency $\omega$, and one "output" photon of frequency $2 \omega$, and occurs in materials that do not possess inversion symmetry. Using a classical nonlinear electron spring model, we know that light-matter interaction results in an electronic polarization $(P)$, which is not just linearly proportional to the electric field (E), but also has contributions from higher powers of $E$ :

$$
\vec{P}(\omega)=\overrightarrow{P_{0}}+\varepsilon_{0} \chi_{e} \vec{E}(\omega)+\chi^{(2)} \vec{E}(\omega)^{2}+\chi^{(3)} \vec{E}(\omega)^{3}+\cdots,
$$

where the second order susceptibility, $\chi^{(2)}$, a third-rank tensor $\left(\chi_{i j k}\right)$, is responsible for the SHG phenomenon of doubling the frequency $(\omega)$. Schematics for our SHG experimental setup in the transmission geometry is shown in figure 2.10. 


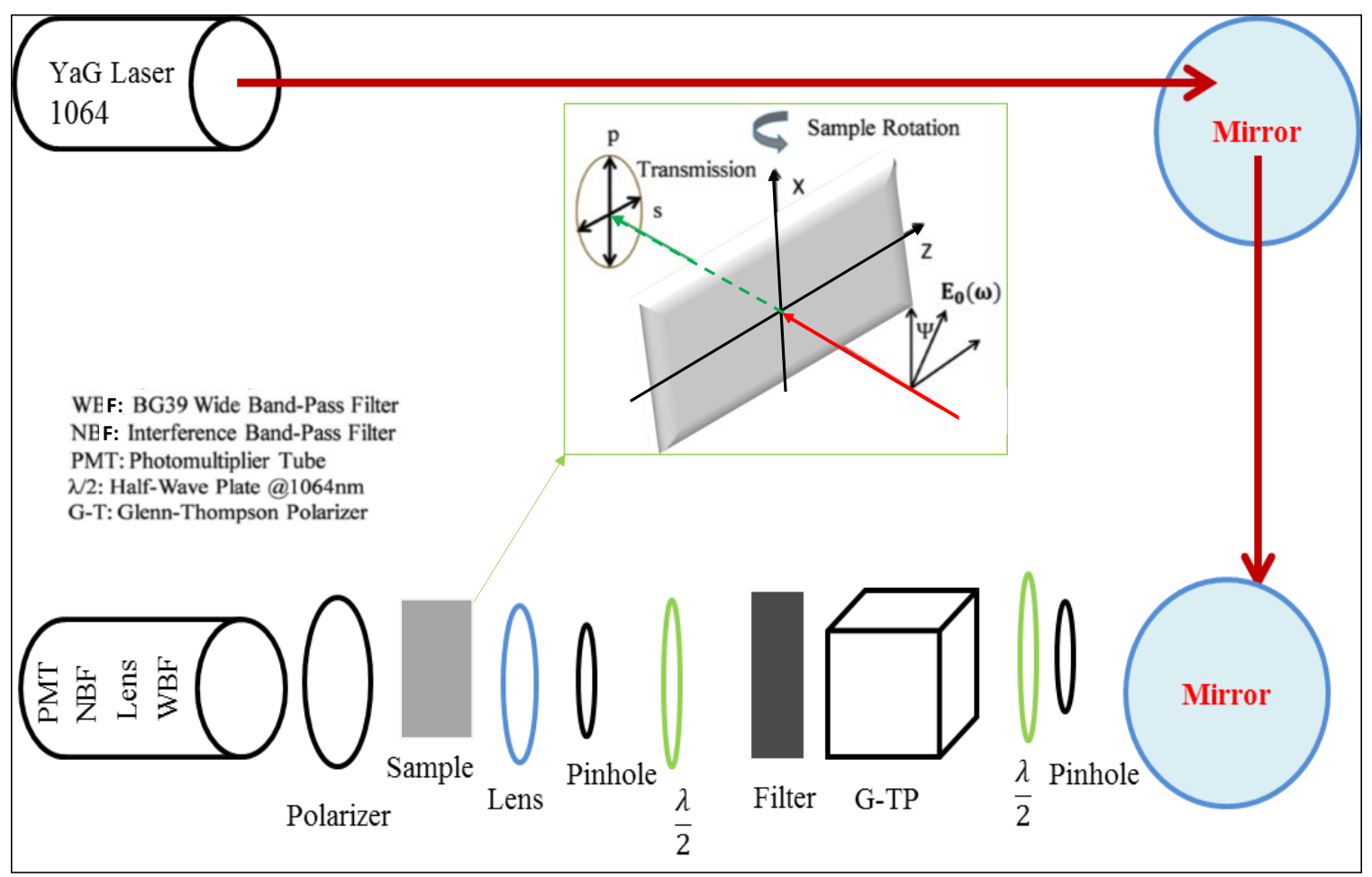

Figure 2.10 Schematics for SHG experimental set up transmission geometry.

Although the ferroelectric property is clearly seen in as-casted PVDF-TrFE films (figure. 2.8 (b-c)), which is indeed a property of the $\beta$ phase, the SHG efficiency in PVDFTrFE is weak. Hence an alignment of the $\beta$ phase by electrical poling (details of poling of PVDF-TrFE are discussed in chapter 6), as schematically shown in figure 2.11 , is required for the observation of SHG for thicker films (approx. $500 \mathrm{~nm}$ films). By monitoring the SHG signal, one can thus estimate the extent of the alignment of the $\beta$ phase in PVDFTrFE. For convenience, PVDF-TrFE polymer is represented with uniaxial $6 \mathrm{~mm}$ symmetry. ${ }^{29}$ The relationship between the induced SHG polarization and the applied fundamental field is given by: 


$$
\left(\begin{array}{c}
P_{x^{\prime}}(2 \omega) \\
P_{y^{\prime}}(2 \omega) \\
P_{z^{\prime}}(2 \omega)
\end{array}\right)=\left(\begin{array}{cccccc}
0 & 0 & 0 & 0 & d_{15} & 0 \\
0 & 0 & 0 & d_{15} & 0 & 0 \\
d_{15} & d_{15} & d_{33} & 0 & 0 & 0
\end{array}\right)\left(\begin{array}{c}
E_{x^{\prime}}^{2}(\omega) \\
E_{y \prime}^{2}(\omega) \\
E_{z \prime}^{2}(\omega) \\
2 E_{y \prime}(\omega) E_{z \prime}(\omega) \\
2 E_{x^{\prime}}(\omega) E_{z^{\prime}}(\omega) \\
2 E_{x \prime}(\omega) E_{y \prime}(\omega)
\end{array}\right)
$$

where $\left(x^{\prime}, y^{\prime}, z^{\prime}\right)$ are the Cartesian coordinates of the sample frame, and $E_{x} \cdot(\omega), E_{y^{\prime}}(\omega)$, and $E_{z^{\prime}}(\omega)$ are the incident electric field components oscillating at the incident frequency $\omega$. By expressing the electric field of the sample frame in terms of the laboratory reference and using Eq. (2.2), one can deduce the $p$ and $s$ components of the SHG polarimetry. $p$ polarization is the polarization of the electric field parallel to the plane of incidence and spolarization is the polarization of the electric field perpendicular to the plane of incidence. By using another coordinate axis for the laboratory reference frame and expressing the electric fields and the polarization of the sample reference frame in terms of the laboratory reference frame with $\Psi$ being the angle of incident polarization and $E_{0}$ the magnitude of the incident electric field, the $p$ and the $s$ component of the polarization can be determined ${ }^{30}$ Given that in PVDF-TrFE: $\mathrm{d}_{15}=\mathrm{d}_{31},{ }^{31}$ the intensity of the $p$-component and $s$-component are given by:

$$
\begin{aligned}
& I_{p}=p^{2}=\frac{1}{2}\left|d_{15} E_{0}^{2}\left(\frac{3}{2} \cos ^{2} \Psi+\sin ^{2} \Psi\right)+\frac{1}{2} d_{33} E_{0}^{2} \cos ^{2} \Psi\right|^{2}, \\
& I_{s}=s^{2}=\frac{1}{2}\left(d_{15} E_{0}^{2} \sin 2 \Psi\right)^{2},
\end{aligned}
$$




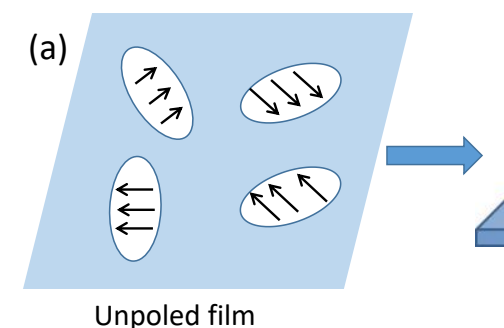

Unpoled film
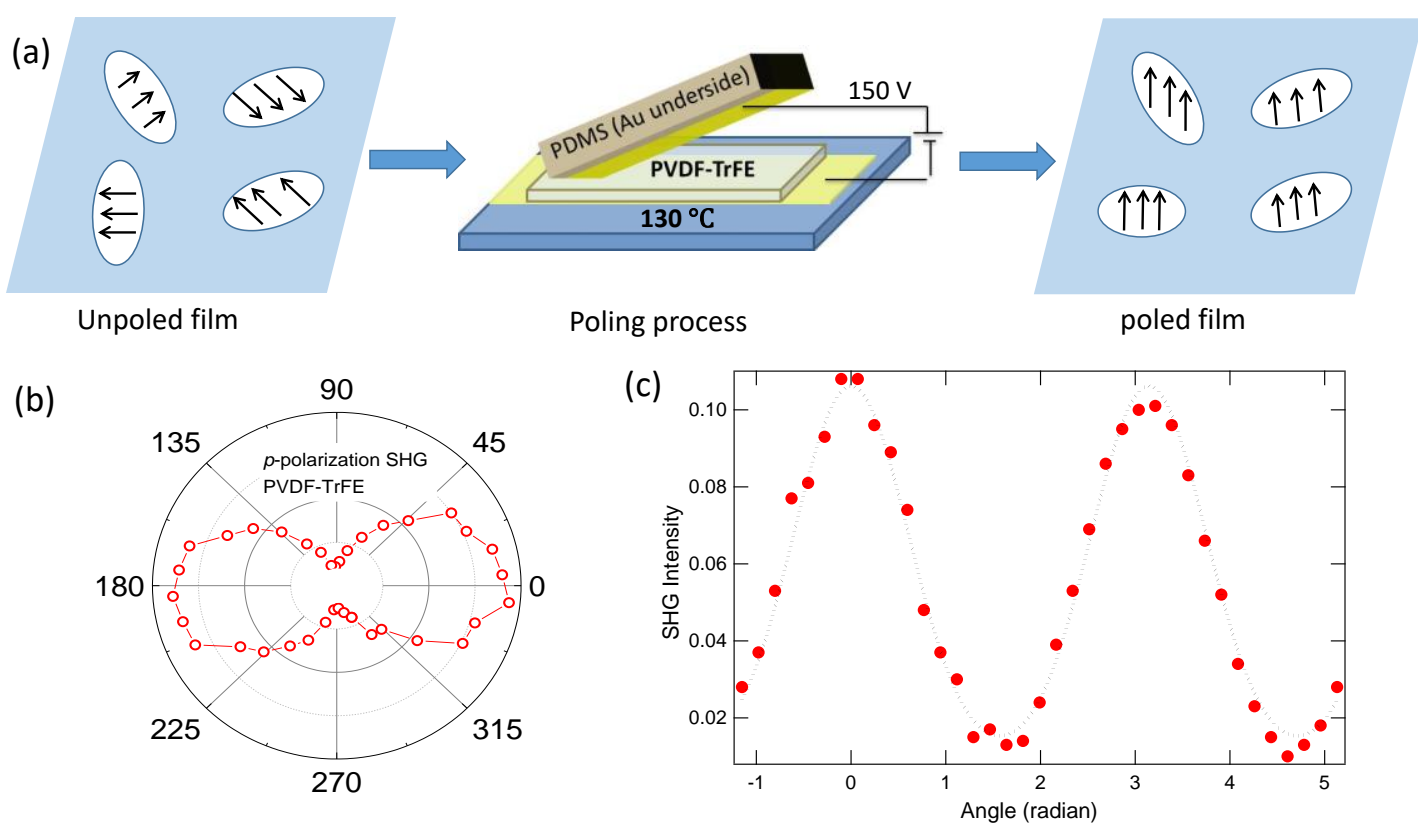

Figure 2.11 (a)Unpoled PVDF-TrFE, a relaxaor polymer has crystalline domains with directed dipole moments in an amorphous matrix. Application of a vertical electric field, while heating the film just above the ferroelectric-paraelectric transition temperatures, results in an alignment of the net dipole moment. (b) Experimental SHG polarimetric response of the p-polarization component from poled PVDF-TrFE film. (c) Filled circles denote the experimental p-polarization SHG data and the black dotted line is a fit to Eq. (2.3).

After electrical poling the PVDF-TrFE layer, the SHG was monitored in transmission geometry. The $p$-polarization is much stronger than the s-polarization, and the intensity was measured as a function of the rotation of the angle of incident polarization, shown in figures 2.10 (b) and (c). By fitting the experimental data with Eq. (2.3) for the $p$ polarization component, we obtain a ratio of the SHG coefficients $d_{33} / d_{15}=2.3$. The experimental data thus confirms the assumed symmetry of PVDF-TrFE. By recording the Maker fringes, Berge et al. determined the SHG coefficients of PVDF-TrFE (70:30) as $d_{33}$ $=0.22 \times 10^{-12} \mathrm{~m} / \mathrm{V}$ and $d_{15}=0.05 \times 10^{-12} \mathrm{~m} / \mathrm{V} .^{31}$ This yields a ratio about two times what we observe. The discrepancy may rise from a different morphology of the film. The thickness 
of the film in our experiment is much lower compared to Ref. ${ }^{31}$, which may yield differences in the ferroelectric domains. The SHG experiments therefore confirm the structure of PVDF-TrFE as well as indicate the extent of poling. The poled films are further utilized in FETs for monitoring transport, as discussed in chapter 5.

\subsubsection{Non-polar (non-ferroelectric) dielectrics}

Devices discussed in this work also make use of other organic and organicinorganic stacked dielectrics. The other two organic insulators used in this research are poly-4-vinylphenol (PVP) and CYTOP. PVP is one of the most widely used insulators for organic FET devices because of its ability to provide high carrier mobility and stable devices. ${ }^{32}$ The monomer is an ethylene with one of the carbons containing a 4-phenol group. The "4" in 4-phenol refers to the position of the $\mathrm{OH}$ group on the phenyl group. The " 1 " position is the carbon connected to the ethylene group and the carbons are numbered around the 6-carbon ring in order to give the lowest number to the other attached groups. " 4 " is directly opposite the "1" position. The backbone of the polymer chain is formed by these ethylene groups. Both printed and spin-casted PVP thin films have been used in this work. The other dielectric used in this study is CYTOP. It is an amorphous fluoropolymer with high optical transparency and excellent electrical and surface properties. It has good solubility in certain fluorinated solvents due to its amorphous nature. The advantage of this fluorinated solvent is it does not interact with the organic materials allowing the ability to fabricate CYTOP dielectric films on top of the organic semiconductor. This allows us to fabricate dual gate FETs. Figure 2.12 (a) and (c) show molecular structure of PVP and CYTOP, respectively. Figure 2.12 (b) shows optical image of printed PVP film on glass. 
(a)

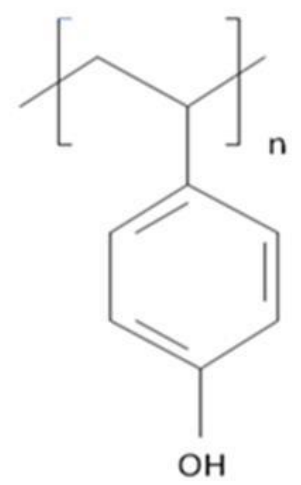

(b)

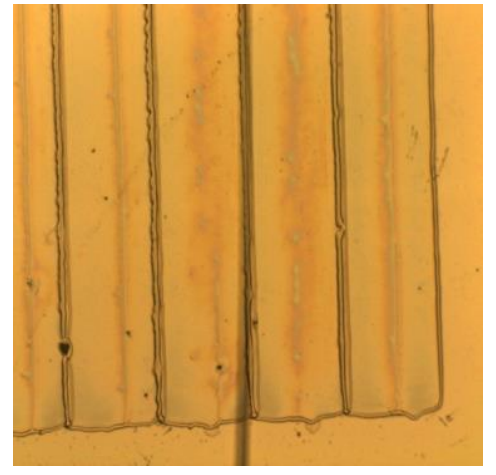

(c)

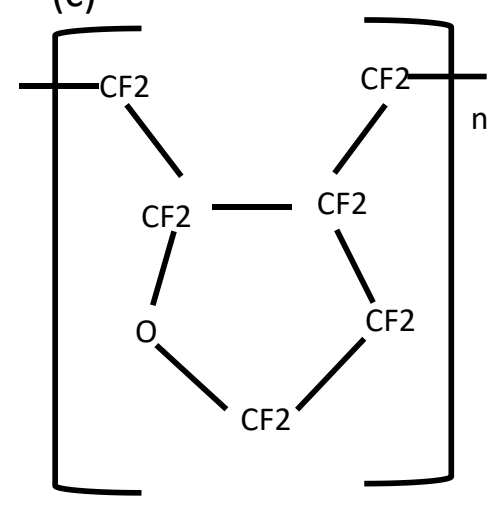

Figure 2.12 (a) Molecular structure of PVP (b) Optical image of printed PVP (c) Molecular structure of CYTOP.

Inorganic dielectrics used in this work are $\mathrm{SiO}_{2}$ and $\mathrm{Al}_{2} \mathrm{O}_{3} \cdot \mathrm{Al}_{2} \mathrm{O}_{3}$ has higher dielectric constants that that of $\mathrm{SiO}_{2}$, which is useful for FET device applications because a high dielectric constant means an increased capacitance, which translates to a larger magnitude of charge accumulated in the conduction channel at lower applied gate voltages. It is important to have low operating voltages in order to reduce the power consumption of transistors. It has been shown that the use of $\mathrm{SiO}_{2}$ as the insulating layer in pentacene FETs leads to an increase in voltage required to form a conducting channel in the semiconductor layer, a decrease in mobility, and an increase in operating voltage. ${ }^{33}$ In this work the use of inorganic and non-ferroelectric dielectrics are mainly for a comparative study with the polymer ferroelectric dielectric PVDF-TrFE. Table 2-I lists of dielectrics used along with their corresponding dielectric constant. 
Table 2-I Dielectric constant of dielectrics used in this research.

\begin{tabular}{cc}
\hline Dielectrics used & Dielectric constant \\
\hline $\mathrm{SiO}_{2}$ & 3.9 \\
$\mathrm{Al}_{2} \mathrm{O}_{3}$ & 10 \\
$\mathrm{PVP}$ & 3.9 \\
PMMA & 3.5 \\
CYTOP & 2 \\
PVDF-TrFE & 8.3 (at $300 \mathrm{~K})$ \\
\hline
\end{tabular}

\subsection{Working principle of organic FETs}

An FET is a three terminal electronic device where a field is applied to one terminal, called the "gate", resulting in the formation of a conductive channel between the other two terminals known as the "source" and "drain". FETs are comprised of multiple thin films. Two basic components are the semiconductor and the dielectric layer. When a gate potential is applied, it induces, via an electric field through a dielectric film, an accumulation of charge carriers (electrons or holes) in the semiconducting layer. These accumulated mobile charges then act as the conductive channel between the source and drain electrodes. In an organic FET, the semiconducting layer is a thin film of an organic semiconductor. The separation between source and drain is called "channel length" and the width of the source or drain electrode is called "device width".

Figure 2.13 shows various types of geometries of organic FETs that are used in this work. In an FET, the gate electrode is separated from the semiconducting layer by a dielectric. The source and drain electrodes must be in direct contact with the semiconductor as they will have to inject and extract charge carriers, respectively. The gate electrode 
(along with its dielectric) may be placed above or below the semiconductor film. Based on the placement of the source, drain, and gate electrodes, the FETs are classified as follows: (a) bottom gate top contact (BGTC), (b) bottom gate bottom contact (BGBC), (c) bottom contact top gate (BCTG) and (d) dual gate, which either works as a top gate or a bottom gate device.

(a) BGTC

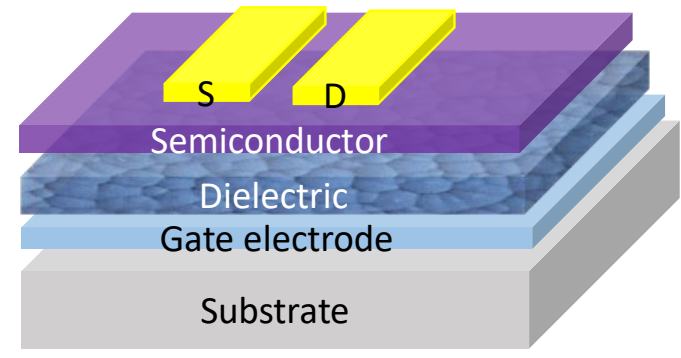

(c) BCTG

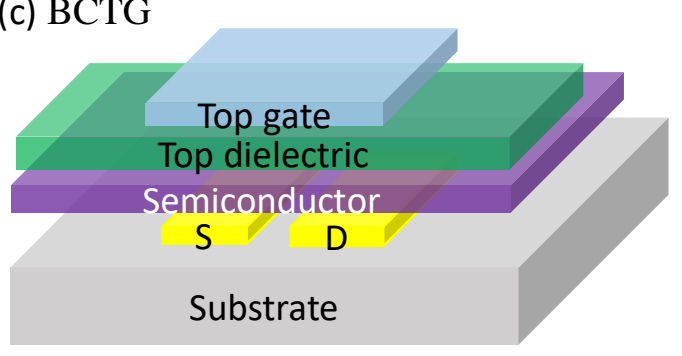

(b) BGBC

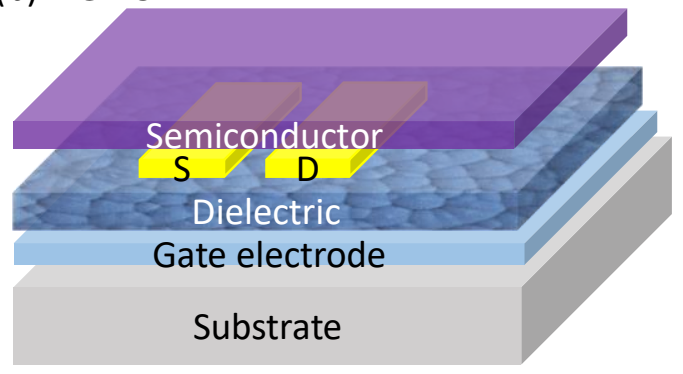

(d) Dual gate

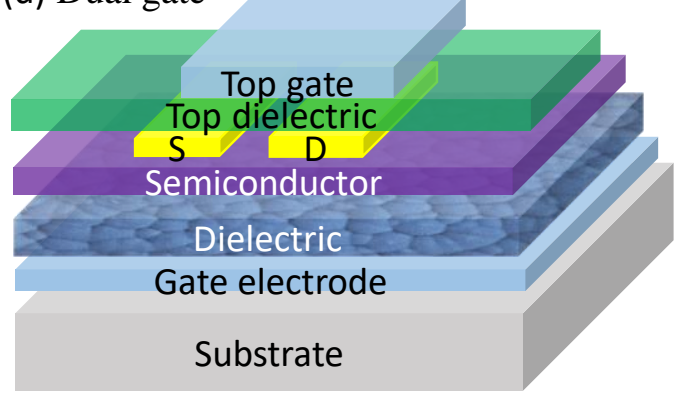

Figure 2.13 Schematics of different FET geometries used in this work. (a) bottom-gate, top-contact; (b) bottom gate, bottom-contact; (c) top-gate, bottom-contact (d) dual gate.

The nature of the metal-semiconductor interface plays a large role in charge transport in organic FETs. A quantity detrimental to transport is the contact resistance which has its origin both at the metal-semiconductor and semiconductor-dielectric interface. An FET operates as a voltage-controlled current source. By applying a voltage at the gate $\left(\mathrm{V}_{\mathrm{GS}}\right)$ across the gate dielectric, and after overcoming the trap states, a sheet of 
mobile charge carriers is induced in the semiconductor that allows the drain current ( $\left.\mathrm{I}_{\mathrm{DS}}\right)$ to flow through the semiconductor when another voltage between the drain-source $\left(\mathrm{V}_{\mathrm{DS}}\right)$ is applied. The source contact is typically grounded.

Unlike the conventional metal oxide silicon FETs (MOSFETs) where the device operates in the inversion mode, organic FETs typically utilize intrinsic semiconductors and therefore operate in the accumulation mode. When a negative gate-source voltage is applied, positively charged carriers are accumulated in the p-type semiconductor near the dielectric interface as shown schematically in figure 2.14 (a). Figure 2.14 (c) shows the energy-level diagrams across the semiconductor/dielectric interface of organic transistors and along the carrier channel with accumulation of positive charge carriers and the transport of positive carriers. Figure 2.14 (b) and (d) are counterpart if carriers are negative. WF in the figure refers to work function which implies the minimum energy required to extract an electron from a metal electrode. IP is ionization potential meaning the minimum energy required to extract electrons available at the HOMO level of the semiconductor and EA is electron affinity which is the minimum energy required to extract electrons available at the LUMO level of the semiconductor, respectively. 
(a)

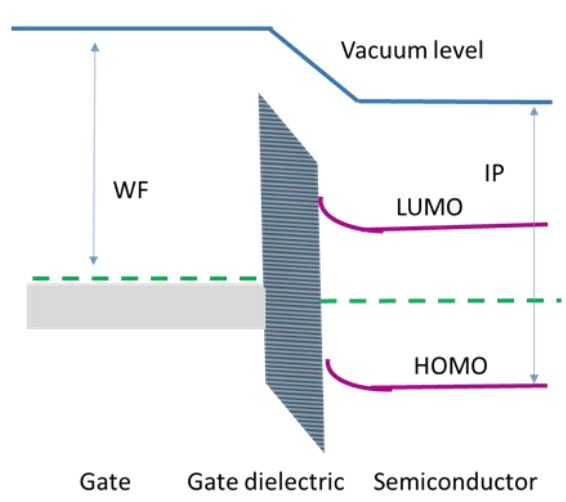

(c)

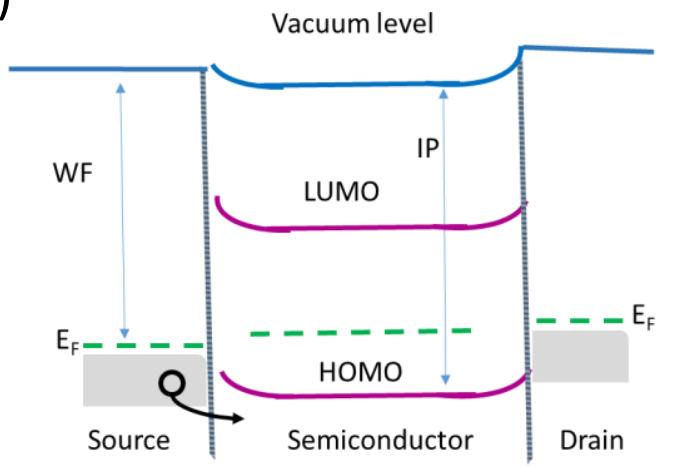

(b)

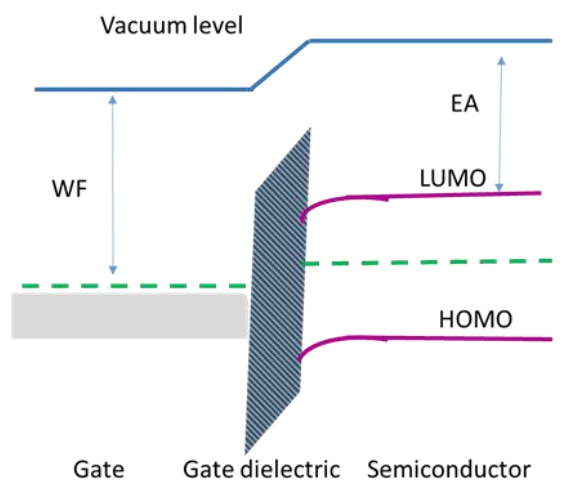

(d)

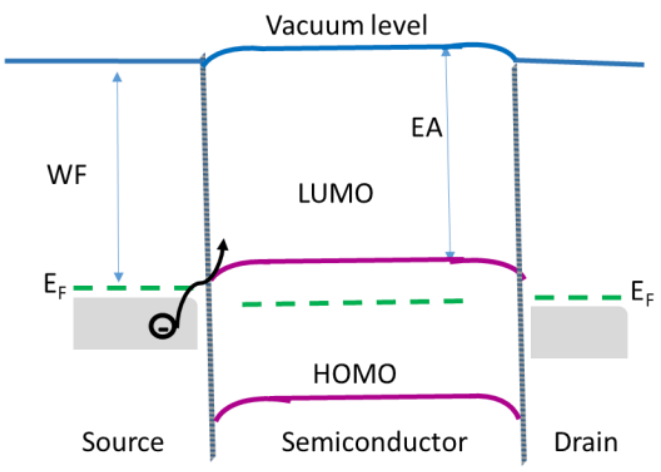

Figure 2.14 Energy-level diagrams across the semiconductor/dielectric interface of organic transistors and along the carrier channel. Diagrams were drawn similar to Ref.14. (a) Accumulation of positive charge carriers for negative gate-source voltages (p-channel transistor). (b) The accumulation of negative carriers for positive gate-source voltages (n-channel transistor). (c) The transport of positive carriers from the source through the semiconductor to the drain in the case of a p-channel transistor. (d) The transport of negative carriers from the source through the semiconductor to the drain in the case of an n-channel transistor.

In p-channel transistors the transfer of negative charges into the semiconductor is blocked due to the large energy difference between the Fermi level of the contact and the LUMO of the semiconductor, and in n-channel transistors the transfer of positive charges is blocked by the energy barrier between the contact and the HOMO of the semiconductor. ${ }^{14,15}$ 
Though the physics of charge transport in organic FETs is different from that in MOSFETs, the current-voltage characteristics can be described with the same formalism. We use the long channel approximation where the horizontal electric field between the drain and the source is very small compared to the vertical electric field induced by the gate. When a potential is applied at the gate, a charge proportional to the capacitance of the dielectric is induced at the dielectric-semiconductor interface. Traps within the semiconducting layer are filled before the mobile charges begin to accumulate and form the conducting channel. The gate voltage at which the conducting channel is formed is referred to as the threshold voltage $\left(\mathrm{V}_{\mathrm{Th}}\right)$ similar to the gate voltage required to induce a strong inversion layer in a MOSFET. ${ }^{34}$ The amount of charge along the channel varies linearly as a function of the position when a drain-source bias is applied.

Provided that $\mathrm{V}_{\mathrm{GS}}>\mathrm{V}_{\mathrm{Th}}$, an increase in $\mathrm{V}_{\mathrm{DS}}$ but less than $\left(\mathrm{V}_{\mathrm{GS}}-\mathrm{V}_{\mathrm{Th}}\right)$ results in charges being accumulated and in an increase in $\mathrm{I}_{\mathrm{DS}}$. The device is said to be in the linear regime. If $\mathrm{V}_{\mathrm{DS}}$ is further increased such that $\mathrm{V}_{\mathrm{DS}}=\mathrm{V}_{\mathrm{GS}}-\mathrm{V}_{\mathrm{Th}}$, the channel is "pinched off" and a further increase in $V_{D S}$ will not result in an increase in IDs. In this case, the vertical electric field in the channel close to the drain is insufficient to cause the accumulation of charge; IDS is said to be saturated. Figure 2.15 shows the schematics for operating regimes of an organic FET along with $\mathrm{I}_{\mathrm{DS}}$ versus $\mathrm{V}_{\mathrm{DS}}$ curves on the right. 
(a)
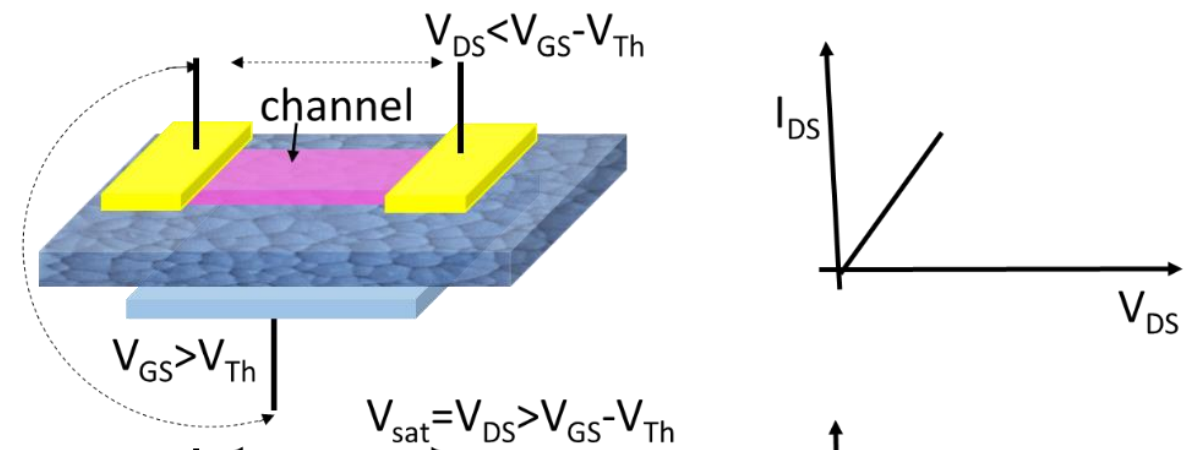

(b)
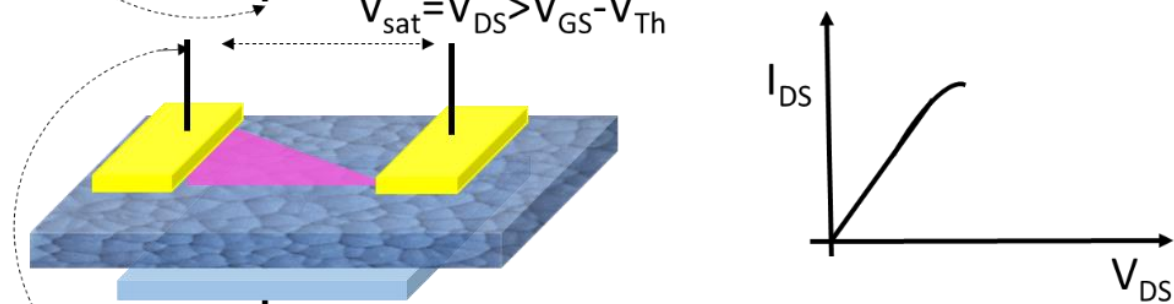

(c)

$$
\mathrm{V}_{\mathrm{GS}}>\mathrm{V}_{\mathrm{Th}}
$$
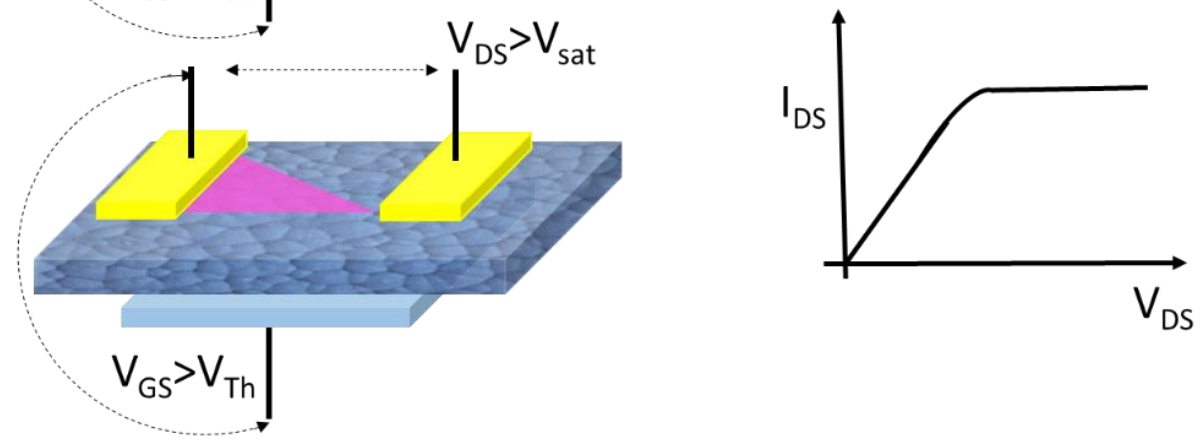

Figure 2.15 Schematic of the operating regimes of an FET. (a) Linear regime. (b) Pinch off. (c) Saturation regime.

The relationship between $\mathrm{IDS}_{\mathrm{DS}} \mathrm{V}_{\mathrm{GS}}$ and $\mathrm{V}_{\mathrm{DS}}$ in the linear and saturation regime are described by the Mott-Shockley equations,

$$
\left.I_{D S}=\frac{\mu_{F E T} W C_{0}}{L}\left(V_{G S}-V_{T h}\right)^{2} V_{D S}-\frac{V_{D S}^{2}}{2}\right),
$$

for $\left|V_{G S}-V_{T h}\right|>V_{D S}$ (linear regime).

$$
I_{D S}=\frac{\mu_{F E T} W C_{0}}{2 L}\left(V_{G}-V_{T h}\right)^{2},
$$


for $\left|V_{G S}-V_{T h}\right|<V_{D S}$ (saturation regime).

In the above equations $C_{o}$ is the gate dielectric capacitance per unit area, $\mu_{\mathrm{FET}}$ is the carrier mobility in the semiconductor, $\mathrm{W}$ is the channel width, and $\mathrm{L}$ is the channel length of the transistor.

Expressions for the carrier field-effect mobility in the linear and saturation regimes can be derived by rearranging equations (2.5) and (2.6) and using the condition $\mathrm{V}_{\mathrm{DS}}=\mathrm{V}_{\mathrm{GS}}$ $-\mathrm{V}_{\mathrm{Th}}$ and $\mathrm{V}_{\mathrm{GS}} \gg \mathrm{V}_{\mathrm{Th}}$ in the saturation regime,

$$
\begin{gathered}
\left(\mu_{F E T}\right)_{\text {Linear }}=\frac{L}{W C_{0} V_{G S}}\left(\frac{\partial I_{D S}}{\partial V_{G S}}\right), \\
\left(\mu_{F E T}\right)_{\text {Saturation }}=\frac{2 L}{W C_{0}}\left(\frac{\partial \sqrt{I_{D S}}}{\partial V_{G S}}\right)^{2},
\end{gathered}
$$

When the $I_{D S}$ (as a function of $V_{G S}$ ) of an organic FET is measured, it can be fit to equations 2.7 or 2.8 allowing for the extraction of device properties such as $\mu_{\mathrm{FET}}$ and $\mathrm{V}_{\text {Th. }}$. The current that flows in alternative paths towards the gate rather than the designated current $\mathrm{I}_{\mathrm{DS}}$ is called the leakage current $\left(\mathrm{I}_{\mathrm{GS}}\right)$. Ideally, it should be zero, but in practice there is a significant amount of leakage current due to the non-ideality of dielectrics. For an optimum FET, leakage current should be several orders of magnitude lower than IDs. There have been recent discussions in the literature whether extracting carrier mobilities using the saturation region from FET transfer curves are appropriate. ${ }^{35,36}$ Several works show that obtaining the mobility from the linear region of the FET transfer curve is a better metric 
for reliable mobilities. However, the linear region is also affected to a greater extent by contact resistance compared to the saturation region, especially for small channel length devices. ${ }^{37}$ A recent molecular-level FET device simulation shows that as long as the energetic disorder of the semiconductor is lower than $2 k_{B} \mathrm{~T}(50 \mathrm{meV})$, the bulk mobility is similar to saturation and linear mobility. ${ }^{38}$

Analysis of current-voltage characteristics of an FET gives the critical device parameters. The behavior of $I_{D S}$ as a function of $V_{D S}$ at various $V_{G S}$ is the output characteristics of an FET. Figure 2.16(a) shows a sample set of output characteristics of an FET based on PVDF-TrFE and TIPS-pentacene. As described above, for a given $\mathrm{V}_{\mathrm{GS}}$, the IDS will increase linearly with $V_{D S}$ until it becomes saturated. As $V_{G S}$ is increased to a higher magnitude, the saturation point will move to a higher value and the saturation current will increase as well. Because the charge carrier density in the semiconductor is a function of the gate-source voltage, the drain current can be modulated by adjusting the gate-source voltage. This modulation of the drain current with the gate-source voltage is quantitatively described by the fundamental field-effect transistor parameter of transconductance, which is denoted by $\mathrm{g}_{\mathrm{m}}$,

$$
g_{m}=\left(\frac{\partial I_{D S}}{\partial V_{G S}}\right),
$$

for constant $V_{D S}$.

The behavior of $I_{D S}$ as a function of $V_{G S}$ at a fixed value of $V_{D S}$, known as the operating voltage, is called transfer characteristics of an FET. Figure 2.16 (b) shows typical transfer characteristics of an organic FET based on PVDF-TrFE and TIPS-pentacene. Here 
we can see the switching behavior clearly. $\mathrm{I}_{\mathrm{DS}}$ increases quadratically with $\mathrm{V}_{\mathrm{GS}}$, quickly taking the transistor from an off state (where drain current reaches a minimum) to an on state. It is seen from equation (2.6) that the square root of $\mathrm{I}_{\mathrm{DS}}$ should be linear with $\mathrm{V}_{\mathrm{GS}}$ in the saturation regime. The square root of $\mathrm{IDS}_{\mathrm{DS}}$ is also plotted when plotting transfer characteristics, as shown in figure 2.16 (b). A linear fit to this region allows for the calculation of FET mobility by using equation (2.8). $\mathrm{V}_{\mathrm{Th}}$ is then obtained from this linear fit equation as the $\mathrm{x}$-intercept. The on/off ratio can also be easily obtained from the transfer curve. The on/off ratio is the ratio of the on current, defined to be $\mathrm{I}_{\mathrm{DS}}$ when $\mathrm{V}_{\mathrm{GS}}$ is equal to the device operating voltage, to the off current. The switch-on voltage marks the gatesource voltage at which the drain current reaches a minimum value. ${ }^{34}$

(a)

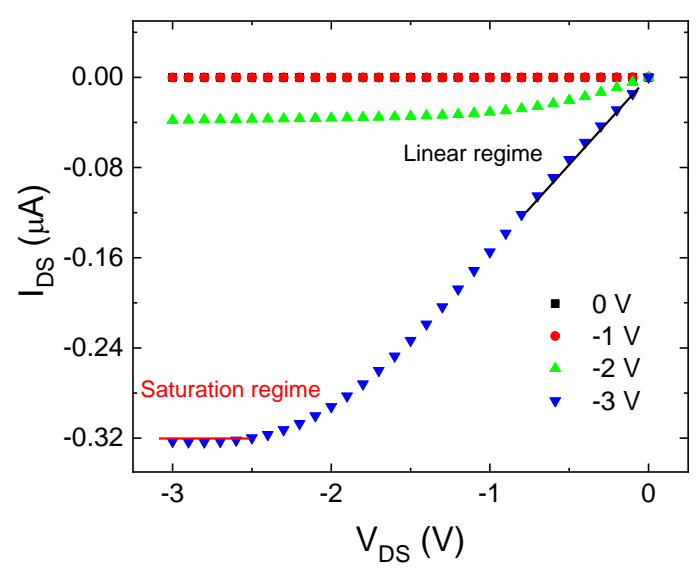

(b)

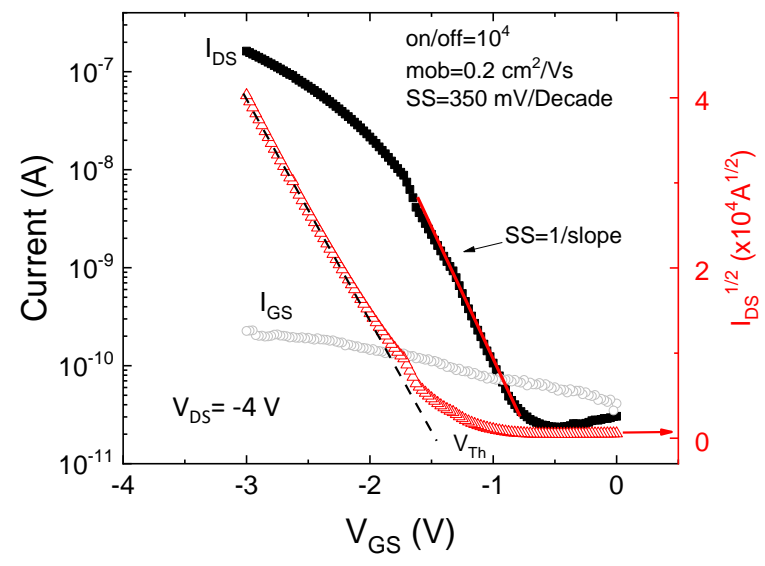

Figure 2.16 Sample (a) output and (b) transfer characteristics of an organic FET. In (a) four output curves corresponding to four gate voltages are shown. The saturation of $I_{D S}$ is clearly seen in each of the curves. In (b) both the $I_{D S}$ and the square root of $I_{D S}$ are plotted. $I_{D S}$ is plotted on a logarithm scale to better show the switching behavior of the FET. The linear behavior of the square root of $\mathrm{I}_{\mathrm{DS}}$ is also clearly seen.

The subthreshold region is described below $V_{T h}$ where IDs depends exponentially on $\mathrm{V}_{\mathrm{GS}}$ in the transfer curve. For the transistor as shown in figure 2.16 the subthreshold 
region extends between the switch-on voltage at $-0.7 \mathrm{~V}$ and the threshold voltage at -1.3 V. In the subthreshold region the drain current is due to carriers that have enough thermal energy to overcome the gate-voltage-controlled energy barrier near the source contact and mainly diffuse, rather than drift, through the semiconductor to the drain contact. ${ }^{34,39}$ The inverse of the slope of the $\log \left(\mathrm{I}_{\mathrm{DS}}\right)$ versus $\mathrm{V}_{\mathrm{GS}}$ curve in the subthreshold region is called subthreshold swing (SS) and is calculated by,

$$
s S=\left(\frac{\partial \log _{10}\left(I_{D S}\right)}{\partial V_{G S}}\right)^{-1}
$$

The subthreshold swing (SS) is determined by the density of trap states at the semiconductor/dielectric interface. Since the dielectric-semiconductor interface for organic semiconductor has a low quality due to noncrystalline nature of semiconductor the SS value is large in organic FETs compared to MOSFETs. 


\section{Experimental methods}

\subsection{Substrate preparation}

The quality of the substrate surface is very critical to any FET performance where the metallic electrodes, dielectric and semiconductor layers are deposited immediately on top of another. A dielectric layer and the nature of the dielectric surface (which depends on the quality of substrate surface) are crucial in determining the ordering of the semiconductor film and controlling the density of charge carrier traps. Much care was given in the surface treatments to improve the surface and interface of thin film deposition. This section contains the substrate preparation recipe that was used for all organic FET fabrication.

Substrates used in this work are glass and $\mathrm{Si}^{++} / \mathrm{SiO}_{2}$, typically of $1 \mathrm{x} 1$-inch size. The glass substrates and $\mathrm{Si}^{++} / \mathrm{SiO}_{2}$ wafers were obtained from Fisher Scientific and Silicon Quest International, respectively. All substrates were first cleaned via an organic cleaning process to remove soluble organic impurities. Generally, 4 pieces of substrates were prepared at one time as we can fit all of them inside the thermal evaporator for semiconductor/electrode deposition. Any loose dust left by the diamond scribe on the substrate surface was quickly washed away by rinsing with isopropyl alcohol. Substrates were then immediately submerged in acetone and ultrasonicated for 20 minutes (Aquasonic model 50HT by VWR scientific products). Exposure to ultrasonic (>20 kHz) waves knocks off and dissolves organic contaminants by causing small bubbles in the acetone to burst. After ultrasonication, the substrates were removed from the acetone bath and quickly rinsed with copious amounts of acetone followed by isopropyl alcohol. All traces of the organic 
solvents were then removed from the substrates by rinsing with deionized water. Glass substrates were then baked in a closed oven (Model $20 \mathrm{GC}$ Lab Oven) at $100{ }^{\circ} \mathrm{C}$ to remove any water residue while $\mathrm{Si}^{++} / \mathrm{SiO}_{2}$ substrates were subjected to further surface treatments.

To remove ionic contaminants and heavier organic residues which could be insoluble in acetone and isopropyl alcohol, $\mathrm{Si}^{++} / \mathrm{SiO}_{2}$ substrates were followed by piranha cleaning. A mixture of 7:3 (by volume) sulfuric acid $\left(\mathrm{H}_{2} \mathrm{SO}_{4}\right)$ to hydrogen peroxide $\left(\mathrm{H}_{2} \mathrm{O}_{2}\right)$ constitutes the piranha. This solution is a highly acidic and a strongly oxidizing agent used to clean organic and ionic impurities off the substrates. Since this reaction is very exothermic, the piranha solution is capable of heating itself to temperatures well above the boiling point of water. While sulfuric acid and hydrogen peroxide are mixed together the acid forces the dissociation of the peroxide via protonation. The resultant atomic oxygen is a highly reactive species capable of aggressively oxidizing carbon-bearing residues. Thus, the piranha aggressively eats away organic residues from the surface of the substrates. Immediately after the organic cleaning and rinsing with water, the substrates were submerged in a heated piranha solution for 5 minutes. To ensure the solution was ready when needed, it was prepared during the organic cleaning step. The prepared solution was then heated on a hot plate (CIMAREC Barnstead Thermolyne) at $125^{\circ} \mathrm{C}$ to maintain the temperature of piranha through the treatment. After five minutes in the heated solution, the substrates were removed using plastic forceps and were thoroughly washed with deionized water. The substrates were then dried with compressed air. The substrate surface is highly hydrophilic in nature, which is a direct result of the strongly protonating piranha solution. It is evident in the way water sheeted across the substrate surface. The substrates were placed in containers and moved to a glovebox for storage. 
After 24 hours the substrate surface was treated with octadecyltrichlorosilane (OTS) solution to produce a self-assembled monolayer (SAM). The substrates that emerge from the cleaning procedure are in a hydrated state, and it is vital to wait long enough (overnight) before beginning the OTS surface modification. Details of the OTS SAM deposition can be found in previous works from our research group. ${ }^{40}$ The OTS solution was prepared in a freshly cleaned and dried glassware. Glassware was cleaned using Liquinox Critical Cleaning Detergent (Alconox Inc.) and dried in a closed oven (Model 20 GC Lab Oven) at $120^{\circ} \mathrm{C}$. The glassware was then allowed to cool to room temperature before use. OTS ( $>90 \%$, Sigma Aldrich) was dissolved in HPLC grade toluene ( $>99.9 \%$, Sigma Aldrich) at a concentration of $0.25 \mathrm{~g} / 10 \mathrm{ml}$ in a small petri dish. The solution was allowed to age 3 minutes, and then each substrate was submerged in the solution for 10 to 30s. The substrate was then submerged in fresh HPLC grade toluene while the remaining substrates were treated in the OTS solution. It should be noted that the insertion of each substrate degrades the OTS solution to the point that it can be only be used for 3-4 substrates. Then all the substrates were subjected to ultrasonication in toluene for $30 \mathrm{~s}$. Finally, the completed substrates were rinsed with isopropyl alcohol and dried with compressed air. After completion of the OTS treatment, the $\mathrm{SiO}_{2}$ surface was clean without any cloudy residue. A successful OTS monolayer formation was confirmed by placing a drop of water on the surface and examining the contact angle the drop forms with the surface. It has been shown that the contact angle should be about $80^{\circ} .{ }^{40}$ A surface with a contact angle less than this is indicative of incomplete OTS coverage. If the above described protocol ever results in incompletely covered substrates during a particular session, it may be modified by dipping the substrates for a longer time (up to one minute). 


\subsection{Thin film fabrication}

\subsubsection{Spin-coating}

Spin-coating is a widely employed technique in the deposition of polymeric thin films onto flexible and non-flexible substrates. This method is used to deposit photoresist in high volume manufacturing companies. The procedure can be summarized as: (a) an amount of a solution is dispensed on the substrate (which is held in place by vacuum chuck) either before substrate rotation, known as static dispatch, or while the substrate is rotating, known as dynamic dispatch; (b) the substrate is rotated at a pre-set speed which may vary between a few hundred to a few thousand rotations per minute (rpm); (c) the solution spreads outwards, resulting in the formation of a thin film. Typically, the substrates, along with the newly formed films, will then be baked at an elevated temperature (often under vacuum) to drive off any remaining solvent left in the film. Some polymeric films are baked at a desired temperature and time to enhance the film qualities like phase and crystallinity. The spin-coating process leaves ample opportunity for customization of different materials, solvents, and substrates. A small change in any of the steps as described above may have drastic implications on film thickness, coverage, and uniformity. The film property depends upon (1) the concentration of the solution, (2) the boiling point of the solvent, (3) spin speed, (4) humidity, and (5) the surface of the substrate. For some polymeric materials, because of their solubility, there may be restrictions on usable solvents and maximum obtainable concentration. For highly hydrophobic substrate surfaces, there may be a limitation to the lowest usable spin-speed, as at low speeds the 
solution may simply form a bead and be flung off the substrate without leaving a film. Heating the substrate just before spin coating was found to be helpful in this case.

Spin-coating, in general, is a non-trivial process, and an individual recipe must be developed for each polymeric material and substrate system by carefully optimizing each of the above described steps. A spin coater inside the nitrogen atmosphere glove box (Specialty coating system version 3.4e Model 6708D) was used for depositing all films in this work. Recipes used for major polymers used in this work are listed in Table 3-I. It has been observed that by following the exact recipe the subsequent films are identical in thickness. As an example, the recipe for a $500 \mathrm{~nm}$ thick PVDF-TrFE film resulted in thickness ranging from $496 \mathrm{~nm}$ to $505 \mathrm{~nm}$. RPM sets how fast the chuck spins during the segment of the cycle, RAMP controls approximately how long the chuck will take to reach the desired speed, and TIME controls approximately how long the chuck will hold that speed. RPM4 sets how long it will take for it to stop. SOLVENT, N2 and COATING were set 0 as these three materials were not dispensed. 
Table 3-I Recipes used for spin coating of major polymers used in this work.

\begin{tabular}{l|lll}
\hline & PVDF-TrFE & CYTOP & PVP/PMMA \\
\hline RECIPE & 0001 & 0002 & 0003 \\
RPM1 & 0300 & 0300 & 0800 \\
RAMP1 & 0002 & 0002 & 0001 \\
TIME1 & 0010 & 0010 & 0005 \\
RPM2 & 0600 & 0600 & 2500 \\
RAMP2 & 0002 & 0002 & 0002 \\
TIME2 & 0003 & 0003 & 0005 \\
RPM3 & 1600 & 2000 & 5000 \\
RAMP3 & 0005 & 0005 & 0005 \\
TIME3 & 0060 & 0060 & 0040 \\
RPM4 & 0001 & 0001 & 0001 \\
SOLVENT & 0000 & 0000 & 0000 \\
N2 & 0000 & 0000 & 0000 \\
COATING & 0000 & 0000 & 0000 \\
\hline
\end{tabular}

The dielectric layers of PVDF-TrFE dissolved in the solvent N,Ndimethylformamide (DMF) were created with different concentrations: 1.8 wt. \%, 2 wt. \% 3.5 wt. $\%, 4$ wt. $\%, 4.5$ wt. $\%$, and 16 wt. $\%$ of PVDF-TrFE. The solution was aged overnight or heated at $80{ }^{\circ} \mathrm{C}$ (typically for higher wt. \%) to completely dissolve the solute. A few drops of the solution were dropped onto Al coated glass slides and spin coated at $1600 \mathrm{rpm}$ for $60 \mathrm{~s}$. The films were vacuum annealed at $135^{\circ} \mathrm{C}$ for an hour and a half to improve the crystallinity. The different concentrations resulted in the thickness of the dielectric film to vary from $20 \mathrm{~nm}$ to $500 \mathrm{~nm}$. The thicknesses of the films were measured 
by a reflectometer (Mprobe, Inc.) and further confirmed by a profilometer and/or by capacitance-voltage measurement.

Poly-4-vinyl phenol (PVP) with average molecular weight (MW) 25,000 g/mol, propylene glycol monomethyl ether acetate (PGMEA) with MW $132.16 \mathrm{~g} / \mathrm{mol}$, and crosslinking agent poly(melamine-coformaldehyde)methylated $84 \%$ solution in 1-butane (PMMF), procured from Sigma Aldrich, were baked at $180{ }^{\circ} \mathrm{C}$ for 1 hour after spin coating or printing. Poly(methyl methacrylate) (PMMA, Mw 996, 000), obtained from Sigma Aldrich, was dissolved in dimethylsulfoxide (DMSO). Typical concentration was 6 wt. \% and the solution was put on a hot plate with a magnetic stirrer for $45 \mathrm{~min}$. This film was baked at $100{ }^{\circ} \mathrm{C}$ for 20 minutes. CYTOP (CTL-809M), bought from Asahi Glass Co. Ltd., Japan was diluted with CT-solv 180 and spin-coated on top of the semiconductor to design top gate FETs. Since the polymer film suffers from cracking at high temperature, the films were baked at $60{ }^{\circ} \mathrm{C}$ for a couple of hours.

\subsubsection{Drop casting}

The drop casting technique is typically employed in the deposition of polymeric thin films onto flexible and non-flexible substrates. It is low cost, consumes little solution, and is an easy method. The procedure could be as simple as dispensing solution onto a substrate and letting the solution spread outwards and dry with a resultant thin film formation. Several techniques and methods have been developed to modify the drop casting method for enhancement of the resultant thin films. Here we discuss the drop casting recipe that was used for solution processable organic semiconductors. 
TIPS-pentacene, procured from Sigma Aldrich, Inc., was used without any purification. We also used TIPS-pentacene provided by Prof. John E Anthony at University of Kentucky particularly for the work reported in chapter 6. It was dissolved in anhydrous toluene and stirred on a hot plate with a magnetic stirrer at $60{ }^{\circ} \mathrm{C}$ for an hour. The TIPSpentacene film is obtained through slow crystallization growth in a solvent-saturated atmosphere. As shown in the schematic (figure 3.1 (a)) the substrate was put inside a Petri dish and positioned at a $2^{\circ}$ angle with respect to the horizontal to achieve the orientation of the crystal axes.

(a)

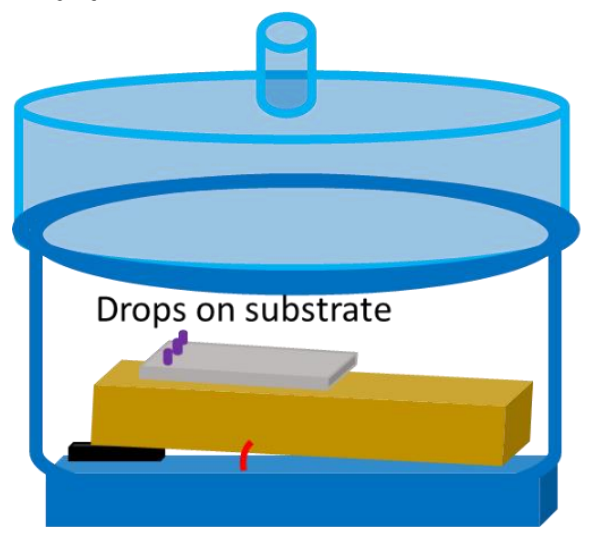

(b)

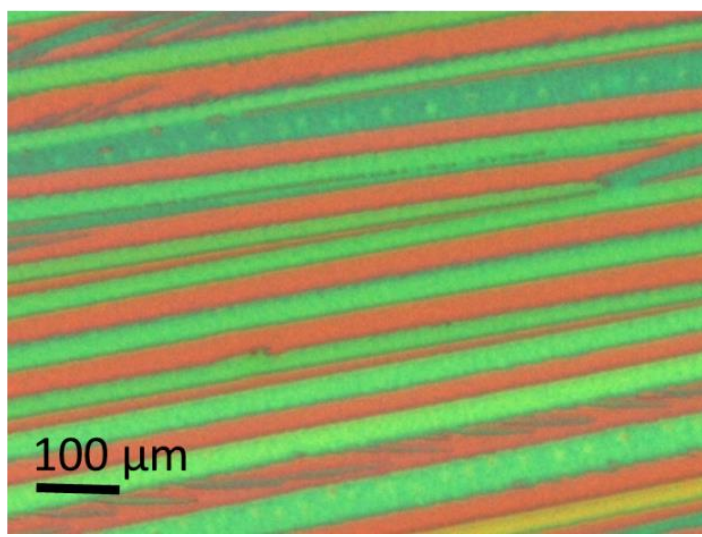

Figure 3.1 (a) Schematics of drop casting method. (b) Drop casted thin film of TIPS-pentacene.

Substrates were placed inside the Petri dish in a manner such that the length of the channel, once the FET device is complete, is parallel to the descending direction of the drying solution. Drop casting is carried out using a pipette with the substrate kept at $50{ }^{\circ} \mathrm{C}$. The lid is put back immediately afterwards to trap the solvent vapor and to create a vaporsaturated condition inside the Petri dish. The lid is removed after 5-10 minutes and excess TIPS-pentacene film was removed from the substrate with just the solvent used for making 
the solution. Particularly for crystalline polymers like TIPS-pentacene, the drop casting method is found very beneficial in device performance over the spin casting method. As shown in figure 3.2 (a), spin casted TIPS-pentacene film has several boundaries with fragmented crystals, while drop casting results in centimeter long strips of thin TIPSpentacene film (figure 3.2 (c)). The FET devices based on such ordered films show a much better device performance than the FETs based on spin casted films, with several order of magnitude higher FET mobility and an on/off current ratio.
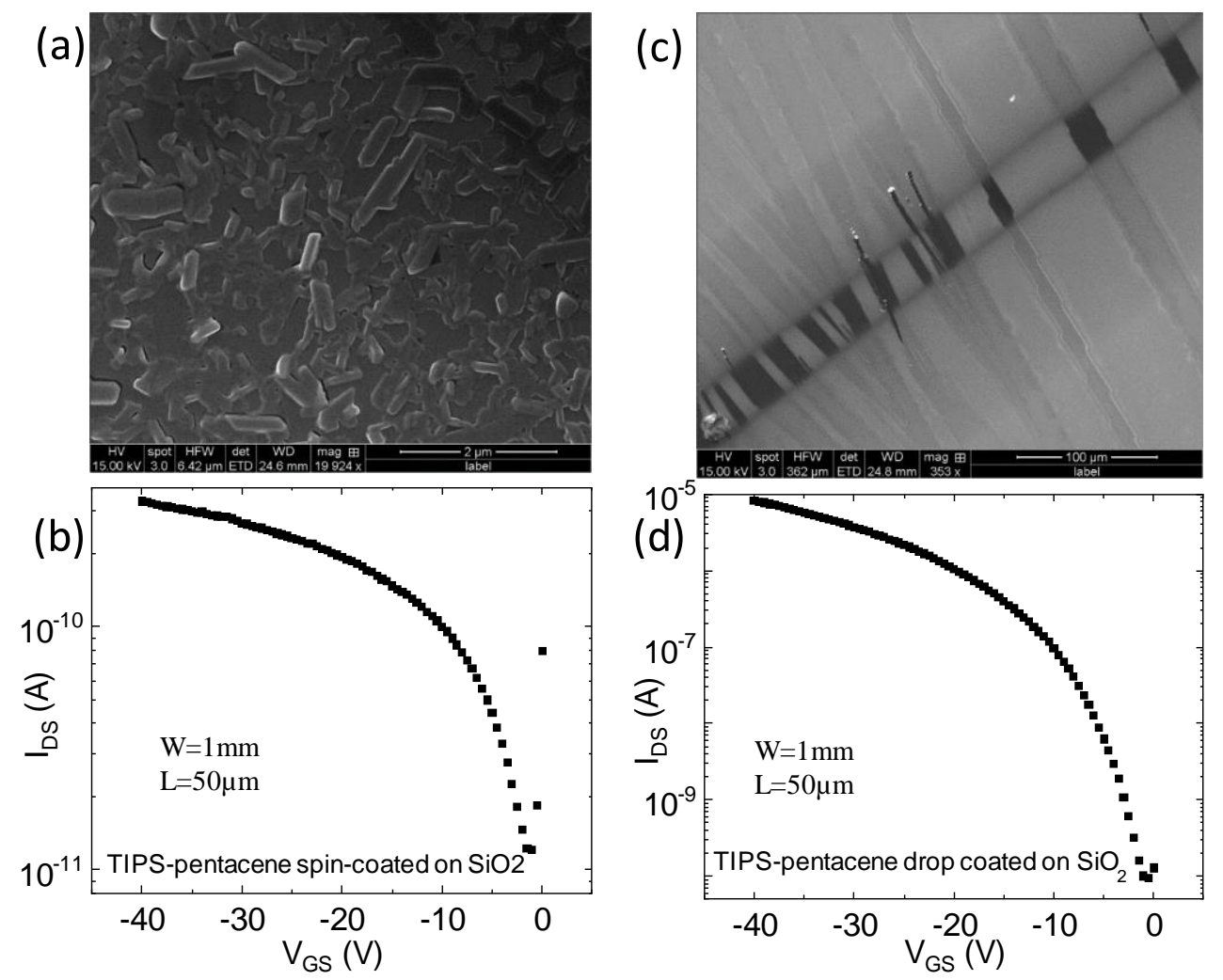

Figure3.2 (a) SEM image of spin coated TIPS-pentacene. (b) Transfer from spin coated TIPS-pentacene FET. (c) SEM image of drop cased TIPS-pentacene. (d) Transfer from drop casted TIPS-pentacene FET. Channel length of both FETs was $50 \mu \mathrm{m}$. 


\subsubsection{Inkjet printing}

Inkjet printing is a technique that creates a designed pattern from a digital image by propelling droplets of ink onto a substrate. A Diamtix materials printer (2831) was used for printing the PVP layer on Al coated glass slides. The procedure can be summarized as: First step is cartridge setting. One the cartridge is filled with cleaning solution and another cartridge is filled with solution to be printed. Then the printhead is attached to the cartridge and installed into the cartridge holder in the printer. Second the ink jetting needs to be checked. One should make sure that all nozzles of the print-head are jetting ink well using the drop watcher. Jetting waveform plays a crucial role for the proper jetting of ink. Figure 3.3 (a) shows an example of a waveform for jetting PVP solution used in this work. Voltage for each nozzle can be adjusted to jet the ink in an equal rate from all nozzles. Figure 3.3 (b) shows the drop watcher image of ejection of PVP droplets. Next is setting the printing pattern. There is an option to select an editable desired printing pattern. If the pattern is complicated, then it should be created using other tools like computer-aided design (AutoCAD). Lastly, the origin position needed to be set. Once everything is ready, the desired pattern is printed setting a proper origin on the substrate. Details of the printing can be found in Diamtix materials printer (2831) in manual.

To print PVP, $5 \mathrm{wt} \%$ solution of PVP in PGMEA along with $2 \mathrm{wt} \%$ of crosslinking agent PMMF was heated at $60^{\circ} \mathrm{C}$ for $1 \mathrm{~h}$ before printing. This solution was filtered through a $0.22 \mu \mathrm{m}$ PTFE filter into $10 \mathrm{pL}$ cartridges (from Dimatix) with $21 \mu \mathrm{m}$ diameter nozzles. Optimal substrate temperature, ink temperature, and drop spacing were determined by trial and error. We found that a substrate temperature of $60{ }^{\circ} \mathrm{C}$ and $50 \mu \mathrm{m}$ drop spacing 
improved the jetting of PVP and minimized the number of pinholes in the film. The voltage of each nozzle was adjusted individually so that the drops hit the substrate at the same time. We used a voltage of $20 \mathrm{~V}$. The PVP layer was printed twice to improve the coverage on the substrate. The printer cartridge moves over the substrate from the origin to the end of the pattern in the horizontal direction to print one band. Then the cartridge moves to a point just below the origin (in the vertical direction) and prints another band in the horizontal direction. This process was continued until the defined pattern was fully formed. There were striations in the PVP film in the direction of printing, which dictates the growth direction of solution processed organic semiconductors such as TIPS-pentacene. After printing, the substrates were moved to a nitrogen environment and annealed at $180{ }^{\circ} \mathrm{C}$ for one hour.

(a)

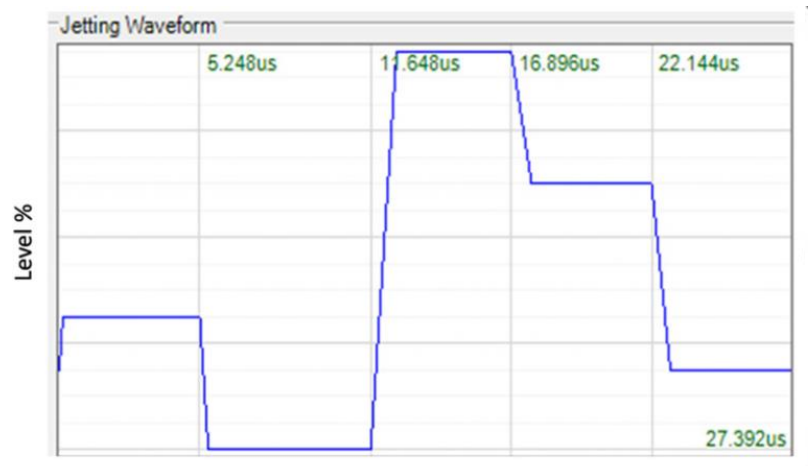

(b)

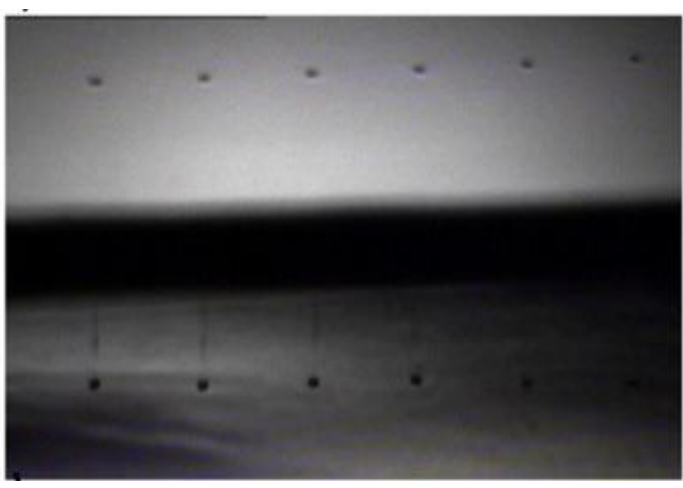

Figure 3.3 (a) The jetting waveform used for printing PVP. (b) Drop watcher image of ejection of PVP solution.

\subsubsection{Thermal evaporation}

Thermal evaporation is a technique where a solid source is heated to the vapor point which then condenses on the target substrate. This process is a form of physical vapor 
deposition and only useful for those materials that have a melting or sublimation temperate much less than their decomposition temperature. The process may be summarized as heating the solid by placing it in a boat made of a high melting point metal (or in a crucible) until individual molecules or atoms have enough thermal energy to escape from the solid, travel from the source to the substrate, and deposit on it, creating a film. These evaporated particles should travel from the source to the substrate without colliding with ambient molecules. So, the evaporation process must occur in vacuum.

In this work we used an MBraun thermal evaporation system where a typical vacuum of $\sim 10^{-5}$ mbar is obtained via Pfeifer vacuum turbomolecular pump backed by a rotary vane pump (Edwards) referred to as the roughing pump. The roughing pump is used to back the turbomolecular pump by maintaining a suitable exhaust pressure $\left(\sim 10^{-1} \mathrm{mbar}\right)$. Evacuation in the MBraun system begins by using the roughing pump to pump the chamber down to 200 mbar, at which point the turbomolecular pump starts and accelerates appreciably after the roughing pump has pumped the chamber down to $10^{-1} \mathrm{mbar}$. The evaporation chamber itself contains resistively heated sources, a substrate holder, and quartz microbalance thickness monitors as shown in figure 3.4 (a). A tungsten boat is used for the evaporation of aluminum and gold, a Molybdenum boat is used for the evaporation of silver and calcium), and a quartz crucible is used for the evaporation of organic semiconductors nestled by a metal bracket. An SQC-222 co-deposition controller is used to control the deposition process. A process may contain multiple layers and a layer can have multiple films of different materials. In our research, gold (Kurt J. Leskar, 99.99\% purity) was evaporated at a rate of $\sim 0.3 \AA / \mathrm{s}$ to a thickness of $50 \mathrm{~nm}$ for source and drain contacts and aluminum at a rate of $\sim 0.5 \AA / \mathrm{s}$ to a thickness of $60 \mathrm{~nm}$ for gate electrodes. 
The average time for evacuation and evaporation for metal deposition was $\sim 40$ minutes. Major parameters used in metal deposition (gold and aluminum) and semiconductor deposition (pentacene) are listed in table 3-II. The recipe for pentacene worked well for DNTT deposition, resulting in a different film thickness.

(a)

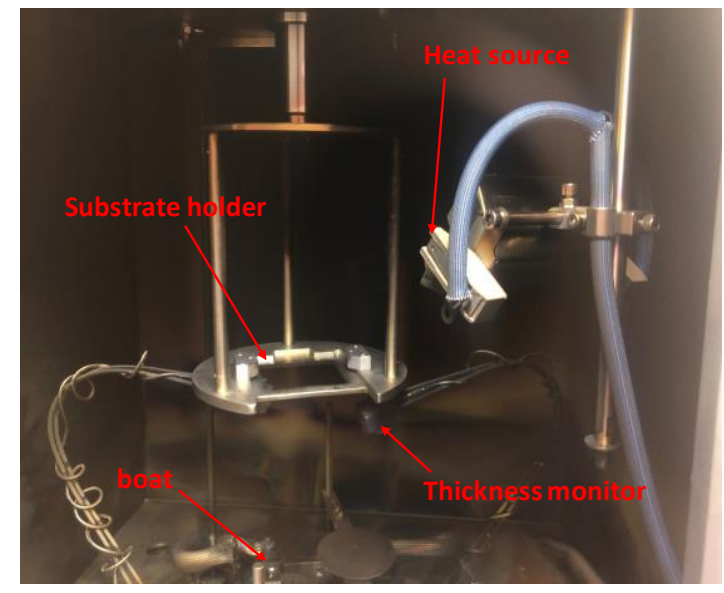

(b)

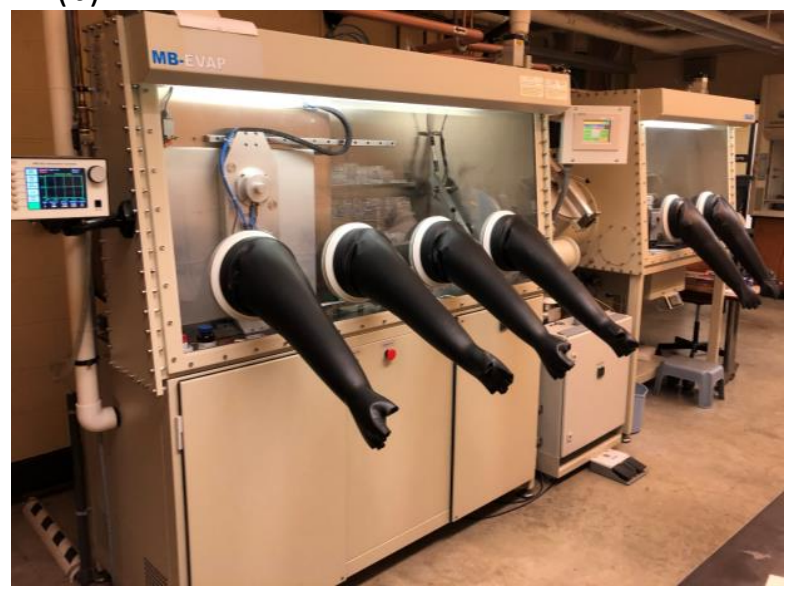

Figure 3.4 Picture of (a) inside thermal evaporation chamber (b) MBraun glove box with thermal evaporator in left box and spin coater in right box. 
Table 3-II Major parameters used in the process to deposit semiconductors and metal electrodes using thermal evaporator.

\begin{tabular}{llll}
\hline Parameters & Pentacene/DNTT & Gold & Aluminium \\
\hline Initial rate & $0.3 \mathrm{~A} / \mathrm{s}$ & $0.3 \mathrm{~A} / \mathrm{s}$ & $0.5 \mathrm{~A} / \mathrm{s}$ \\
Final thickness & $0.5 \mathrm{kA}$ & $0.5 \mathrm{kA}$ & $0.6 \mathrm{kA}$ \\
Max power $(\%)$ & 20 & 30 & 27 \\
P Term & 5 & 5 & 5 \\
I Term(Sec) & 5.0 & 5.0 & 5.0 \\
D Term(Sec) & 0 & 0 & 0 \\
Density(gm/cc) & 2.25 & 19.3 & 2.73 \\
Zfactor & 3.260 & 0.381 & 1.080 \\
Rmp1 power $(\%)$ & 5 & 15 & 15 \\
Ramp 1 Time () & $0: 00: 30$ & $0: 00: 30$ & $0: 00: 30$ \\
Soak 1 Time & $0: 01: 00$ & $0: 01: 00$ & $0: 02: 00$ \\
Rmp2 power $(\%)$ & 7.5 & 20 & 27 \\
Ramp 2 Time & $0: 00: 30$ & $0: 00: 30$ & $0: 00: 30$ \\
Soak 2 Time & $0: 01: 00$ & $0: 01: 30$ & $0: 01: 30$ \\
\hline
\end{tabular}

Isolated FET devices were accomplished by evaporating the metal through a shadow mask that was cut such that it is the negative of the final desired metal pattern. The shadow mask is firmly clamped to the substrate before evaporation to ensure an accurate reproduction of the pattern. Masks made of thin molybdenum, which has the lowest coefficient of thermal expansion of all common metals ${ }^{41}$, were manufactured by Jordan Valley Innovation Center (JVIC, Missouri State University, Springfield, MO) using 
designs made by the author in the AutoCad software package. Another mask purchased from Ossila (Evaporation Stack for Low Density OFETs) was also used for some devices. Some masks were designed and made by using aluminum foil by the author. Sample mask designs are shown in figure 3.5 for (a) gate electrode and (b) source drain electrodes. The mask resulted in devices with channel lengths of $50-200 \mu \mathrm{m}$ and widths of $0.5-2 \mathrm{~mm}$, giving transistor width to length ratios (W/L) of 10 to 40. Small circular structures ranging 250- 1500 micron in diameter are used for capacitor devices. The masks were designed in such a way that the source and drain were connected by a long channel with larger pads to facilitate external connection in a cryostat for temperature dependent measurements. Figure 3.5 (c) shows a complete device structure with FETs and capacitor devices based on drop casted TIPS-pentacene on $\mathrm{Si}^{++} / \mathrm{Al}_{2} \mathrm{O}_{3}$ substrate.

(a)

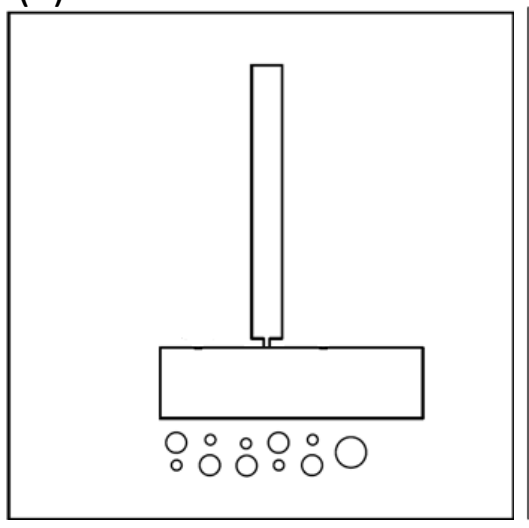

(b)

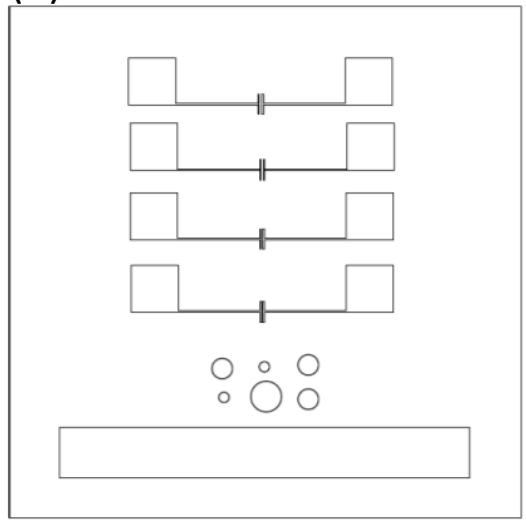

(c)

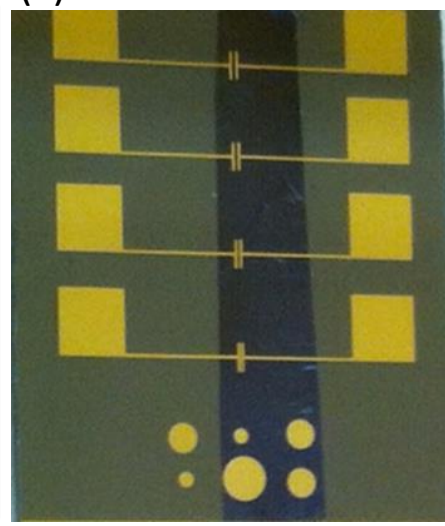

Figure 3.5 Sample mask design used for patterning top contacts for organic FETs and capacitors. (a) Gate electrode. (b) Source drain electrodes. (c) Optical images of FETs and capacitor devices. 


\subsection{Characterization}

\subsubsection{Surface morphology}

Surface morphology of the fabricated thin films was examined using atomic force microscopy, scanning electron microscopy, and contact angle measurements.

\subsubsection{Atomic force microscopy}

In an atomic force microscope (AFM), a cantilever with a very fine tip is used to probe the surface of materials with a resolution on the atomic scale. This technique monitors the change in force between the tip and the surface using an optical probe and by dragging a very sharp tip at the end of a flexible cantilever across the surface of the sample. The tip is moved by a piezoelectric system with high precision while its position is recorded using a laser focused on the back of the cantilever whose reflection is incident on a photodiode detector that transforms the information to a 2D image. AFM can be performed in 3 different operating modes: (1) contact mode where the force on the tip is held constant as it drags across the surface and this force is monitored by the laser positioning system to create the image. It is commonly used for hard surfaces; (2) non-contact where the tip hovers over the surface and interacts using van der Waals and other weak forces; (3) tapping mode; the tip is in contact with the surface only occasionally. In the tapping mode the cantilever is provided a constant driving force where it oscillates near a resonance frequency. The amplitude of oscillations is constantly monitored by the laser/photodiode system. We have employed the tapping mode for all AFM images presented in this work as our samples are soft organic compounds. Typical cantilever specifications we used had a spring constant of $0.1 \mathrm{~N} / \mathrm{m}$, a $28 \mathrm{kHz}$ resonant frequency, a length of $22 \mathrm{~mm}$, a width of 
$42 \mathrm{~mm}$, and a $0.9 \mathrm{~mm}$ thick aluminum reflection coating. The typical specifications of the tips are a conical shape with $15 \mathrm{~mm}$ height and $8 \mathrm{~nm}$ radius. These tips were purchased from Nanoscience Instruments.

Figure 3.6 (a) shows an AFM image from drop casted TIPS-pentacene. This shows uniform surface of TIPS-pentacene crystals orienting in one direction. Unidirectional orientation helps us to position the source and the drain electrodes in such a way that the orientation of TIPS-pentacene ease the carrier flow between the electrodes in an FET. The AFM image is also analyzed to get thickness of the TIPS-pentacene. Figure 3.6 (b) shows AFM images from spin coated PVDF-TrFE where we can see ferroelectric domains like structure varying less than micron to several micron in size are observed. The AFM images are very similar to SEM images as shown in section 3.3.1.2.
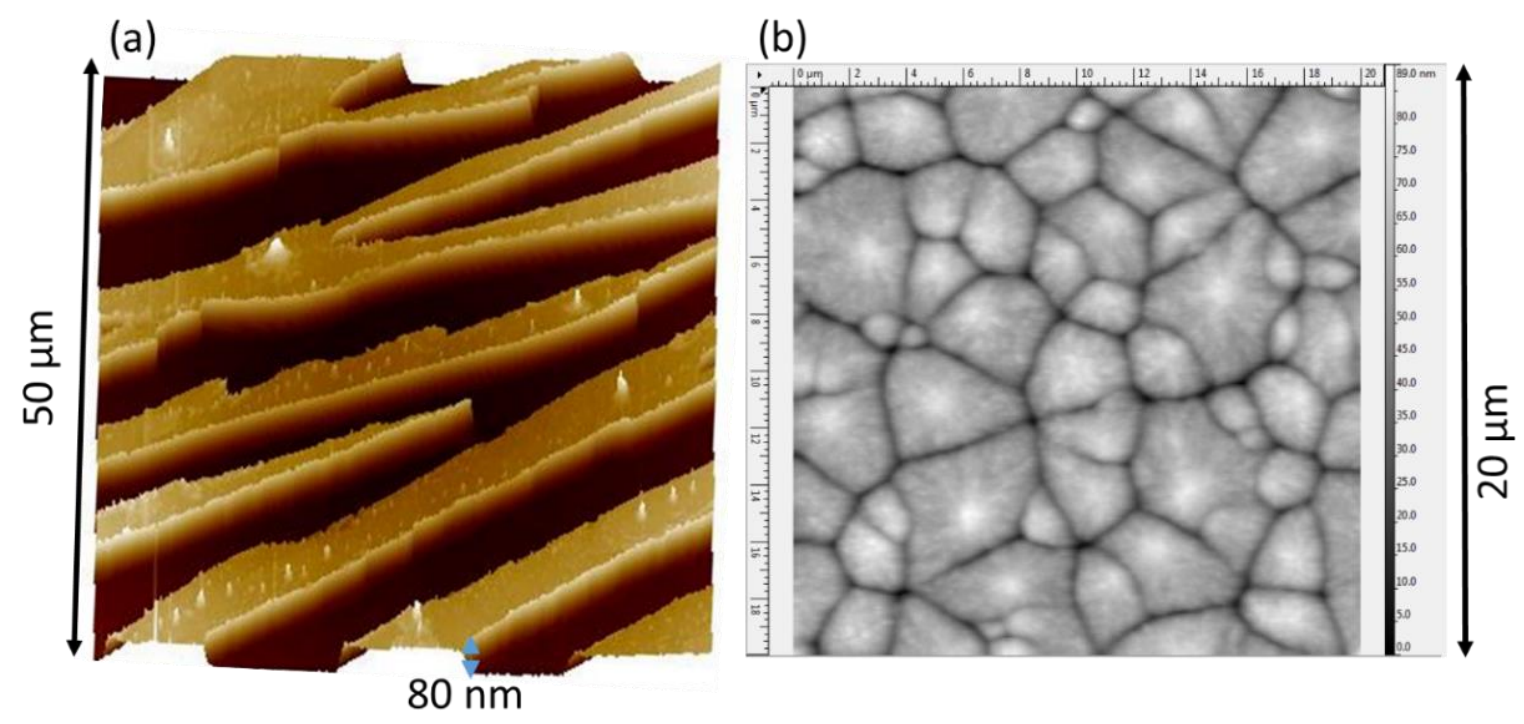

Figure 3.6 (a) AFM images from drop casted TIPS-pentacene. (b) Spin-coated PVDF-TrFE 


\subsubsection{Scanning electron microscopy}

A scanning electron microscope (SEM) uses a non-invasive, nondestructive technique often employed to examine the surface characteristics of materials in the nanometer length scale. Here electrons are focused on the surface of the sample and they interact with the sample. The scattered electrons are finally collected by a detector to form an image. SEM is a sensitive tool to probe topography as well as the composition of the sample surface. It consists of (1) an electron source, most commonly a field emission gun or tungsten filament, that emits electrons in a beam. (2) One or two condenser lenses that govern the intensity of the electron beam. (3) An objective lens that focuses the electron beam on the surface of the sample. (4) Scanning coils that control the placement of the beam. (5) An electron detector connected to the display. To limit the interaction of electrons with ambient gas molecules, SEM is generally carried out under vacuum conditions and the vacuum strength is adjusted according to the nature of the scanning surface. A charging effect (found in insulating or poor conducting samples) tends to trap electrons that do not escape from the sample surface and can cause bright spots in the images. This prevents accurate reproduction of topography and limits the SEM performance.

We used the Electron Microscopy (EM) Core facility at the University of Missouri, Columbia and the SEM facility at the Department of Physics, University of the Western Cape, South Africa. Low-voltage, secondary-electron micrographs of the film surface morphology were collected with a Zeiss Auriga field-emission gun-scanning electron microscope (FEG SEM) at an accelerating voltage of $1 \mathrm{keV}$ as well as a FEI Scios Dual 
Beam focused ion beam SEM (FIBSEM) at an accelerating voltage between $1 \mathrm{kV}$ and 5 $\mathrm{kV}$. The thin films remained uncoated with the surfaces cleaned in the microscope chamber using a nonreactive nitrogen plasma (XEI Scientific Evactron decontaminator) for 10 min. This ensured an effective removal of hydrocarbon contamination on the thin-film surface and minimal radiation damage during analysis. Figure 3.7 shows SEM images from spin coated PVDF-TrFE on aluminum coated glass with different accelerating voltage.

(a)

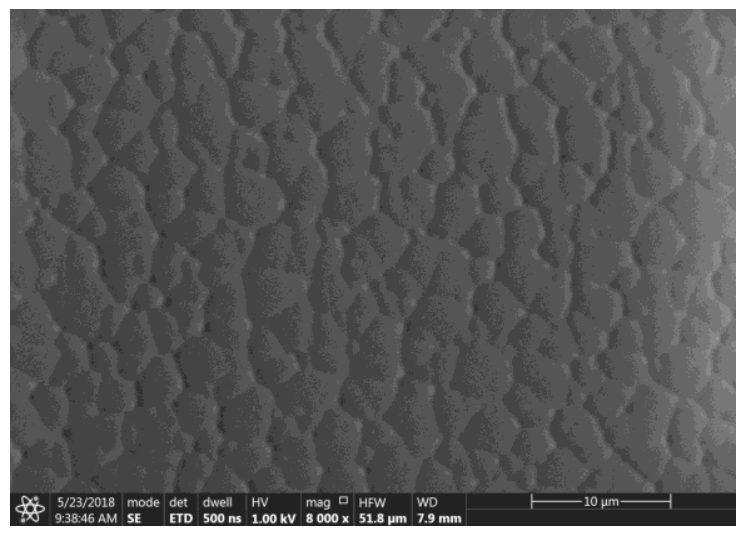

(b)

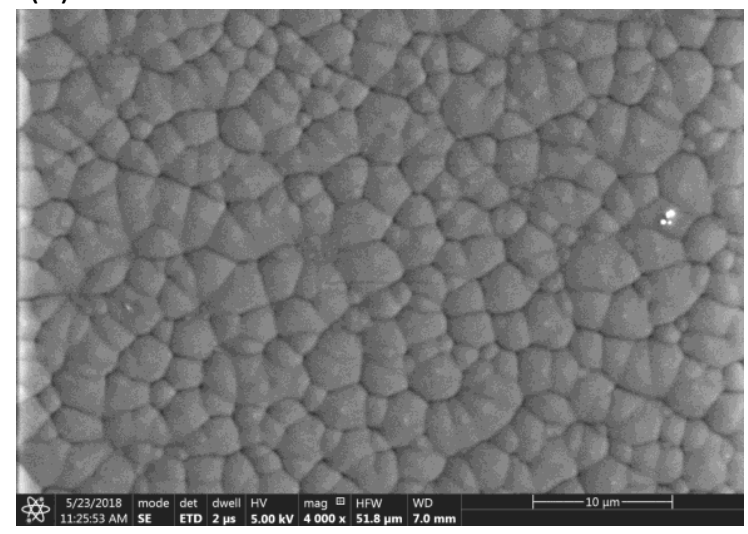

Figure 3.7 SEM images from spin coated PVDF-TrFE on aluminum coated glass with (a) $1 \mathrm{kV}$ (b) $5 \mathrm{kV}$ accelerating voltage.

\subsubsection{Contact angle measurement}

Contact angle measurement is a powerful tool for measuring the surface energy of surfaces. The Ramé-hart Model 200 Standard Contact Angle Goniometer was used for our contact angle measurement. The DROP image Standard software measures contact angles and surface energy tools such as the acid-base tool, the surface energy tool, the work of adhesion tool and the Zisman's plot tool. Zisman's theory simply defines the surface energy as being equal to the surface energy of the highest surface energy liquid that wets the solid completely. i.e., when a droplet spreads out to its maximum on the surface. Three 
liquids: water, formamide, and diiodomethane (with different surface tension), were used as test liquids. Figure 3.8 shows the contact angle measurement from a printed PVP surface. This measurement was carried out to compare the spin coated versus printed PVP surfaces.
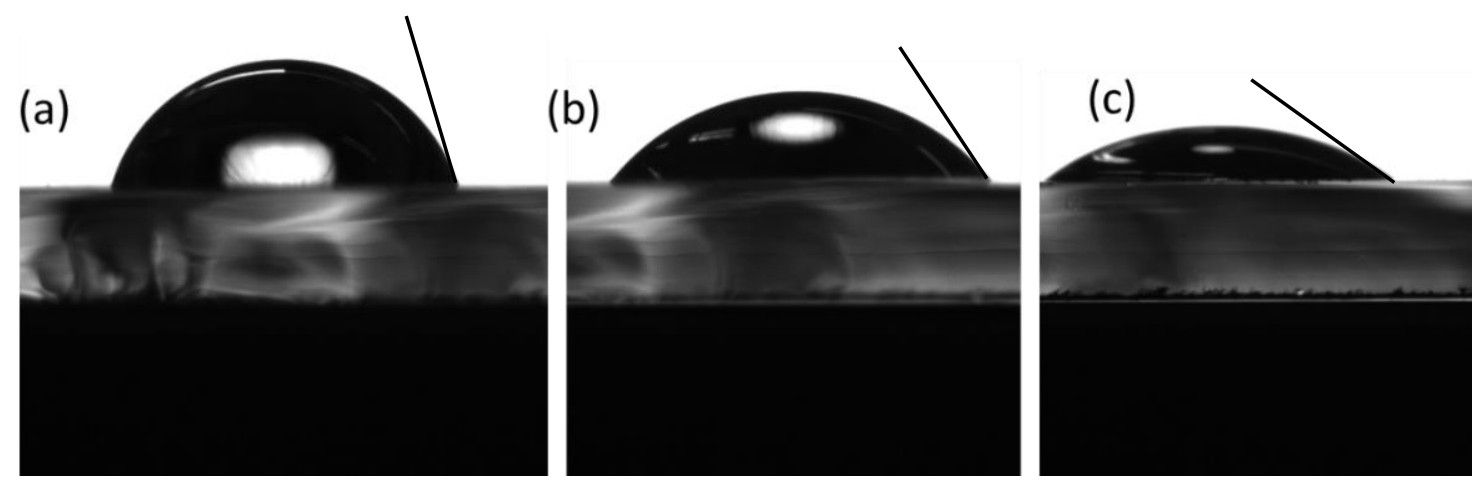

Figure 3.8 Three test liquid drops on the surface of printed PVP (a) water (b) formamide (c) diiodomethane.

\subsubsection{Electrical characterization}

\subsubsection{Capacitance voltage and polarization measurement}

Capacitance versus voltage $(\mathrm{C}-\mathrm{V})$ and capacitance versus frequency (C-f) characteristics were measured from metal-insulator-metal (MIM) and metal-insulator semiconductor-metal (MIS) structures with an HP 4284A LCR meter. Since the capacitance depends upon the thickness and the dielectric of the material, this method was used to verify the thickness of dielectrics using the formula,

$$
t=\frac{\varepsilon_{o} \mathrm{k}}{C o}
$$

where $\mathrm{t}$ is the thickness of film, Co is the capacitance per unit area, $\mathrm{k}$ is the dielectric constant of the materials, and $\varepsilon_{\mathrm{o}}$ is the permittivity of free space.

During the $\mathrm{C}-\mathrm{V}$ and $\mathrm{C}-\mathrm{f}$ measurements, a DC voltage is applied with a small AC voltage signal $(200 \mathrm{mV})$ superimposed while the capacitance is monitored. The application 
of voltage and sensing of capacitance were controlled by a program developed in LabVIEW. The frequency of the signal was usually in the range of $5 \mathrm{kHz}$, at which the dissipating factor was the smallest value for all measurements presented in this work. The DC signal is then swept from positive bias to negative bias with different magnitude depending upon the nature and thickness of dielectric. The number of steps and range of applied bias can be selected in the LabVIEW program. During these sweeps, the capacitance is measured and recorded for each DC voltage applied. During the C-f measurements, a small DC bias $(200 \mathrm{mV})$ is kept constant while the frequency of the AC signal is swept from $10 \mathrm{~Hz}$ to $1 \mathrm{MHz}$ and capacitance is recorded. Figure 3.9 below shows examples of MIM and MIS capacitance characteristics. As shown in figure 3.9 (a) MIS capacitor behavior is characterized by an accumulation of holes at the dielectricsemiconductor interface at negative bias. The interface is between pentacene semiconductor and PVDF-TrFE insulator. A depletion of holes from the dielectricsemiconductor interface is observed at a small negative bias and a deep depletion of holes from the dielectric-semiconductor interfacial region is observed at a positive bias. No inversion is observed due to the long relaxation time of carriers in organic semiconductors. The maximum accumulated capacitance at accumulation regime is used as capacitance per unit area to estimate the thickness of the insulators using Eq. 3.1. 
(a)

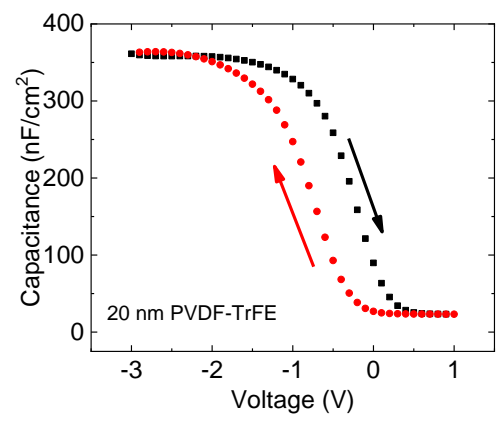

(b)

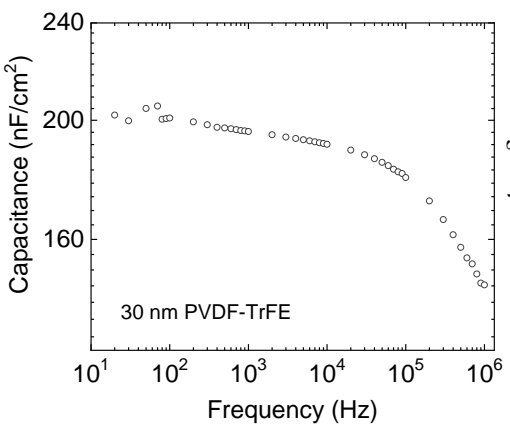

(c)

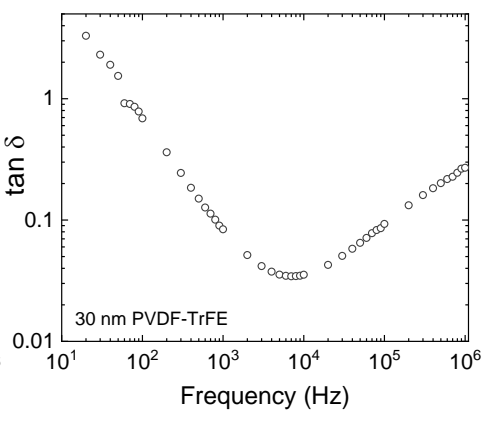

Figure 3.9 (a) MIS Capacitance-Voltage from Al/PVDF-TrFE/pentacene/Au structure. (b) MIM Capacitance-frequency characteristics from Al/PVDF-TrFE/Al structure. (c) Dissipation factor from $\mathrm{Al} / \mathrm{PVDF}-\mathrm{TrFE} / \mathrm{Al}$ structure.

\subsubsection{Current voltage measurement}

The current-voltage (I-V) characteristics of the devices were measured by using Keithley source meters 236, 2400, 2612B, and 4200. Keithley 236 and 2400 are single source meters. For FET characterization we require the sourcing of two voltages (i.e. drain voltage and gate voltage) and the measurement of the respective two currents, so the two units were required to work in tandem. Keithley 2612B is a dual source meter which can be used for sourcing and measurement simultaneously. The 2400 has a resolution of $10 \mathrm{nA}$ while the 236 and 2612B have a resolution of $100 \mathrm{fA}$. Source meter 4200 can measure as low as $1 \mathrm{fA}$.

Room temperature transfer and output characteristics were recorded using a LabVIEW program which set the 2400 to source the desired $V_{G S}$ while using a Keithley 236 to apply the desired $V_{D S}$ as it recorded the current. The program allowed us to choose the desired start and end drain/gate voltage values along with the number of $\mathrm{V}_{\mathrm{DS}} / \mathrm{V}_{\mathrm{GS}}$ points 
to record allowing for a fully automated way to record a full set of output and transfer characteristics.

For temperature-dependent electrical measurements, a dual source meter Keithley 2612B was used. Keithley 2612B has Test Script Processor (TSP) technology embedded inside it and executes complete test programs. All temperature dependent transfer and output characteristics were recorded with TSP. A standardized networking interface RJ-45 LAN crossover cable was used to connect the source meter and computer. As shown in the photograph (figure 3.10) the organic FETs were kept inside a closed-cycle helium cryostat (APD Cryogenics) where the temperature may be varied from $11 \mathrm{~K}$ to $480 \mathrm{~K}$. High vacuum was obtained via TURBOTRONIK NT 10 (Ideal Vacuum) backed by a rotary vane pump (Edwards) before starting the cooler. The temperature was measured using a Lakeshore 330 temperature controller. We allowed enough time to stabilize the temperature (typically 15 minutes from one measurement to another). Most of the devices were measured between $100 \mathrm{~K}$ and $450 \mathrm{~K}$. To test for reversibility, some devices were also measured from high temperature to low temperature. 
(a)

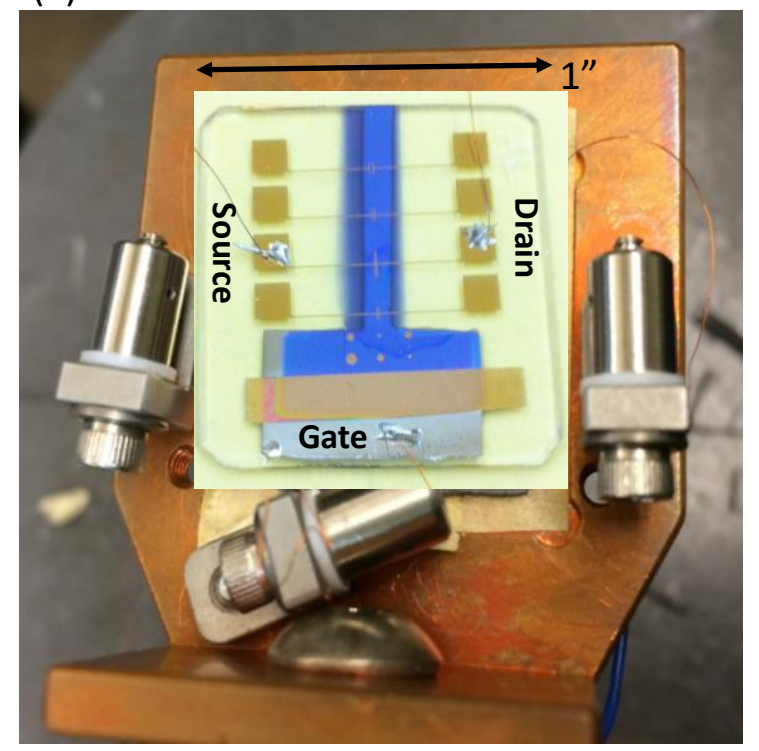

(b)

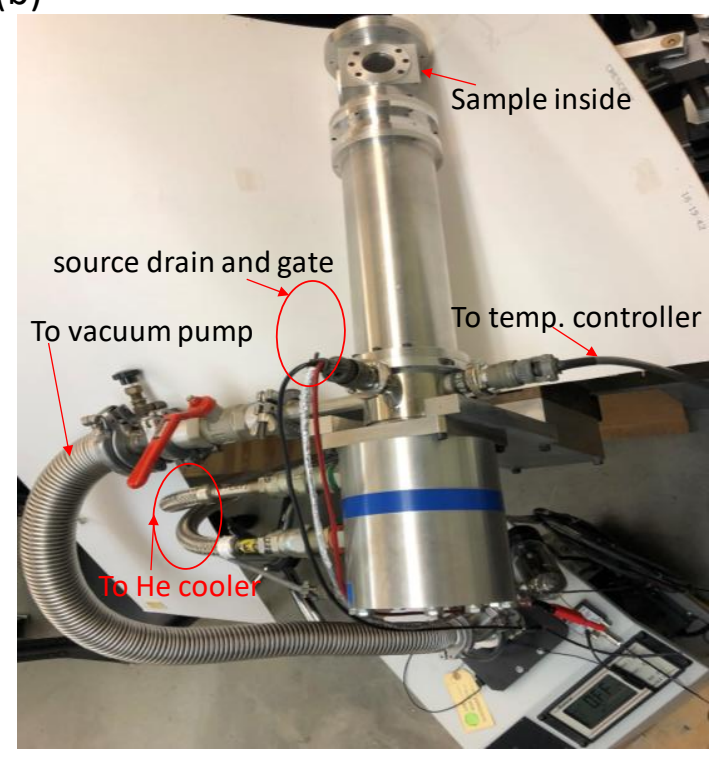

Figure 3.10 Photograph of (a) sample on the stage inside the cryostat (b) Modified closed-cycle helium cryostat (APD Cryogenics). 


\section{The role of Fröhlich polarons in pentacene FETs}

Chapter 2 described the ferroelectric nature of a PVDF-TrFE film which may be used as a gate dielectric layer in organic FETs. In an FET the semiconductor-dielectric interface plays an important role for the transport of charges. Theory reveals the importance of both local and non-local electron-phonon coupling for a comprehensive understanding of the charge transport mechanism. ${ }^{42}$ The dynamic coupling of charge carriers to the electronic polarization at the semiconductor- dielectric interface mainly arises from two effects: the image force due to the polarization discontinuity at the interface and the Coulomb interaction of the charge carriers with the surface phonons of the dielectric, ${ }^{43,44}$ schematically shown in figure 4.1. The Fröhlich polarons reflect a long-range interaction between the charge carriers in the semiconductor and the longitudinal optical (LO) phonons in the dielectric. It is the interactions at the interface that result in a renormalization of the transfer integral for the transport process and manifests itself as reduced charge carrier mobility when the dielectric constant of the gate insulator increases. This chapter explains the role of Fröhlich polarons in pentacene based organic FETs.

\subsection{Introduction}

Considerable efforts have been made in using PVDF and its copolymers with trifluoroethylene (TrFE) as the gate dielectric layer in organic FETs including graphene FETs for memory and other applications. ${ }^{23,45-49}$ Understanding the role of the dielectric layer in interfacial charge transport processes has been equally important. The polaronic nature of the charge carriers is heavily masked by disorder effects. ${ }^{50}$ It has been shown that Fröhlich surface polarons are formed if the gate dielectric is sufficiently polar in molecular 
systems. ${ }^{51}$ A dynamic coupling of the Fröhlich polarons is also observed in ionic liquid gate dielectrics. $^{52}$

Most of the studies describing charge transport in organic materials have been conducted on a variety of organic semiconductors grown on different dielectric insulators where the dielectric constant spans from low dielectric constant to high dielectric constant values. In particular, the dielectric constant changes from 4 to 24 in PVDF-TrFE when the temperature is varied from $200 \mathrm{~K}$ to the ferroelectric-paraelectric phase transition at $390 \mathrm{~K}$ (Tc). ${ }^{53}$ Such a huge increase in dielectric constant of PVDF-TrFE results in stronger interaction between the interfacial carriers and the dielectric polarization. Thus, by using the same organic semiconductor-insulator interface, PVDF-TrFE allows a platform for understanding transport as the polarization strength is tuned from a weak-coupling to strong-coupling regime with changing temperature.

This investigation was motivated by a work where temperature-dependent transport characteristics of P3HT (polythiophene) organic FETs with PVDF and its copolymers as the dielectric layer were investigated. ${ }^{53}$ In this work, we employ pentacene as the organic semiconductor, mainly, so that we can compare our experimental results with theory as well as compare the temperature dependent transport characteristics of pentacene FETs with other non-polar dielectrics. 


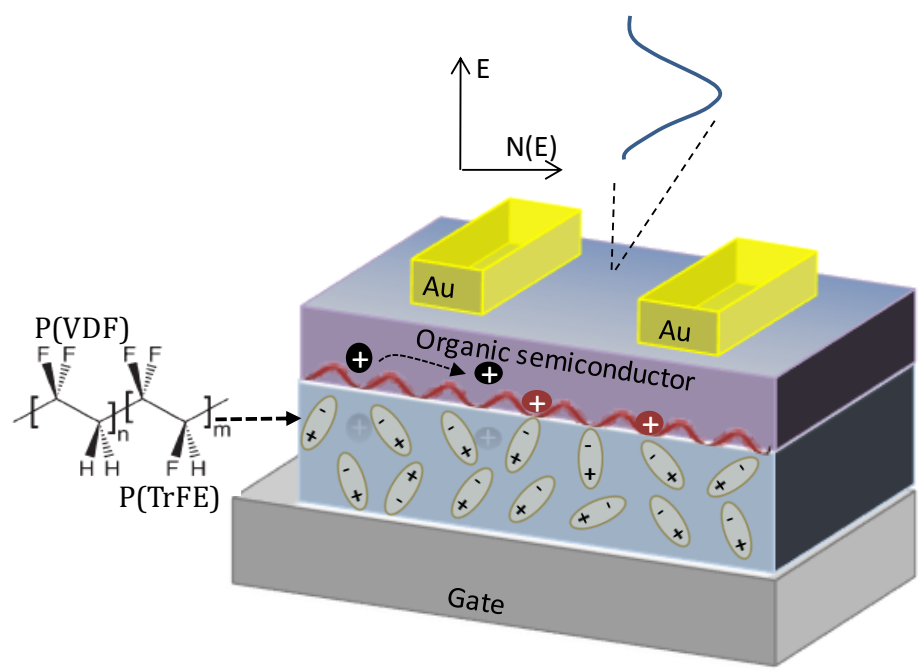

Figure 4.1 Schematic of a ferroelectric-based FET where the dynamic coupling of charge carriers to the electronic polarization at the semiconductor-dielectric interface is illustrated. In addition to the bulk disorder within the semiconductor, there is trapping of charges at the interface.

\subsection{Comparison of polar versus non-polar dielectric}

Thermally activated transport in pentacene organic FETs using non-ferroelectric dielectrics has been observed by many groups. ${ }^{54-56}$ Other acene-based semiconductors also show activated transport with a distinct relationship between the contact-induced film microstructure and charge carrier mobility ${ }^{57}$ Since pentacene has many grain boundaries, rather than a bandlike transport, a thermally activated hopping of charges from site to site within a disorder-induced exponential distribution of states, as proposed by Monroe ${ }^{58}$, is observed. In such a mechanism, the charge carrier mobility, $\mu_{\mathrm{FET}}$, is given by,

$$
\mu_{F E T}=\mu_{o} \exp \left(-\frac{\Delta}{k_{B} T}\right),
$$


where $\Delta$, the activation energy, is the energetic difference between the Fermi energy and the transport level, $\mu_{\mathrm{o}}$ is the mobility in the absence of any trap states, and $k_{B}$ is the Boltzmann constant. Additionally, as the charge carrier density increases, the Fermi energy gets closer to the transport level, easing the transport of charges; thus decreasing $\Delta$. Within the context of transport in organic FETs, the above model is more complex, especially when the polarization field of the dielectric interface needs to be considered. By comparing PVDF-TrFE-based organic FET characteristics to non-polar dielectric organic FETs, the effect of the polarization field can be readily observed.

(a)

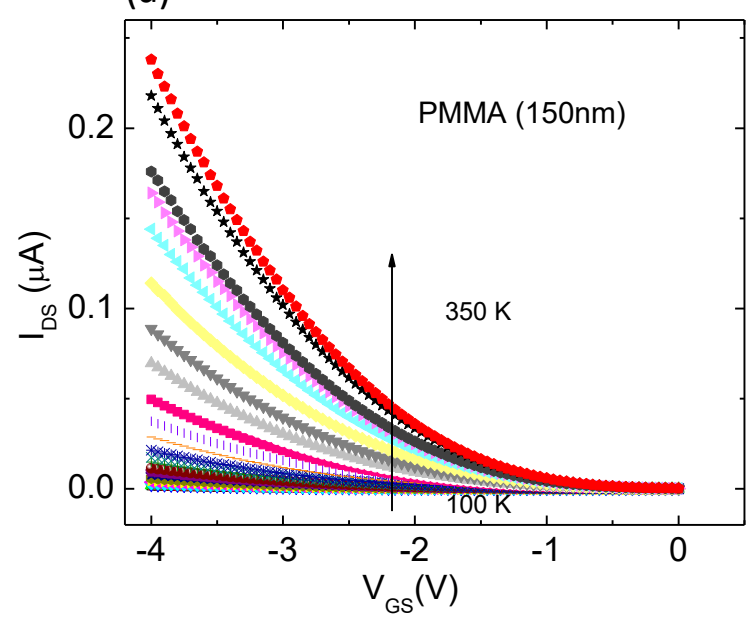

(b)

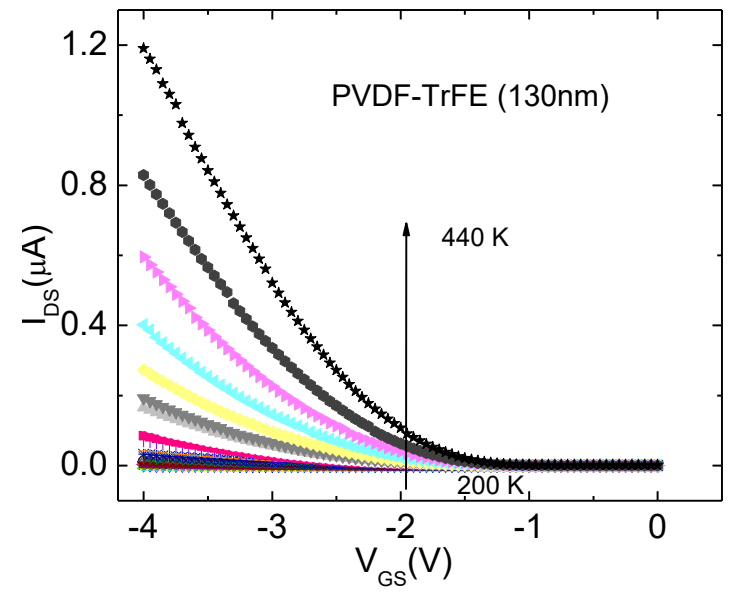

Figure 4.2 (a) Transfer curves at different temperatures from 100K to 350K for a pentacene FET with PMMA as the dielectric layer. (b) Transfer curves at different temperatures from $200 \mathrm{~K}$ to $440 \mathrm{~K}$ for a pentacene FET with PVDF-TrFE as the dielectric layer. In both cases, $\mathrm{V}_{\mathrm{DS}}=-4 \mathrm{~V}$.

Figure 4.2 shows the transconductance curves of PMMA and PVDF-TrFE organic FETs at various temperatures. In both cases, each of transfer curves was measured at a 10 $\mathrm{K}$ interval of temperature in the saturation regime. 
(a)

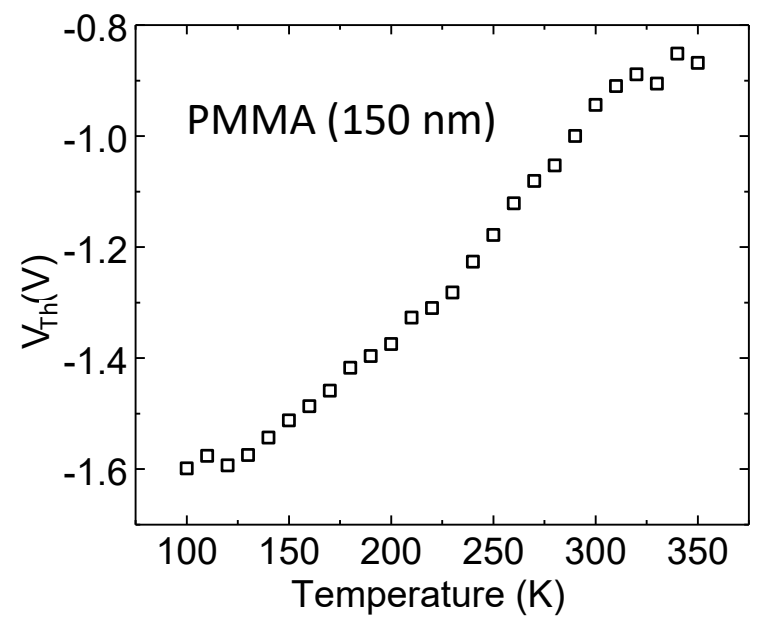

(b)

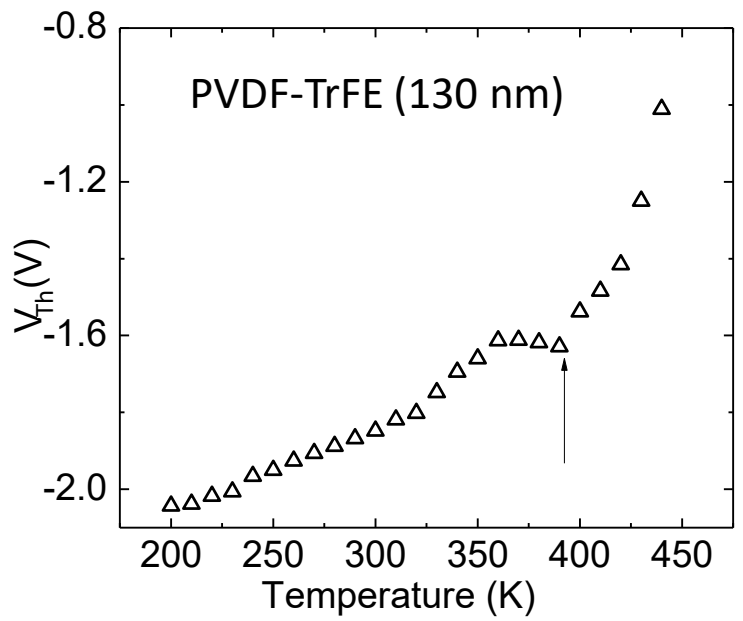

Figure 4.3 Variation of threshold voltage with temperature (a) PMMA FET and (b) PVDF-TrFE TET.

Figure 4.3 show the variation of $\mathrm{V}_{\mathrm{Th}}$ as a function of temperature for PMMA and PVDF-TrFE FETs. These values were derived from the transfer curves shown in figure 4.2. As expected in an activated transport mechanism, the threshold voltage decreases with increasing temperature in both cases; however, the PVDF-TrFE sample shows a discontinuity at the ferroelectric-paraelectric phase transition temperature, beyond which the rate of increase in $V_{T h}$ is much higher compared to in the ferroelectric phase. As we see later, a higher $\mathrm{V}_{\mathrm{Th}}$ can be accounted for by a decrease in the trapped charge density. For the transfer characteristics at each temperature, the gate voltage was swept up to $\mathrm{V}_{\mathrm{DS}}$, which was in the saturation region. 


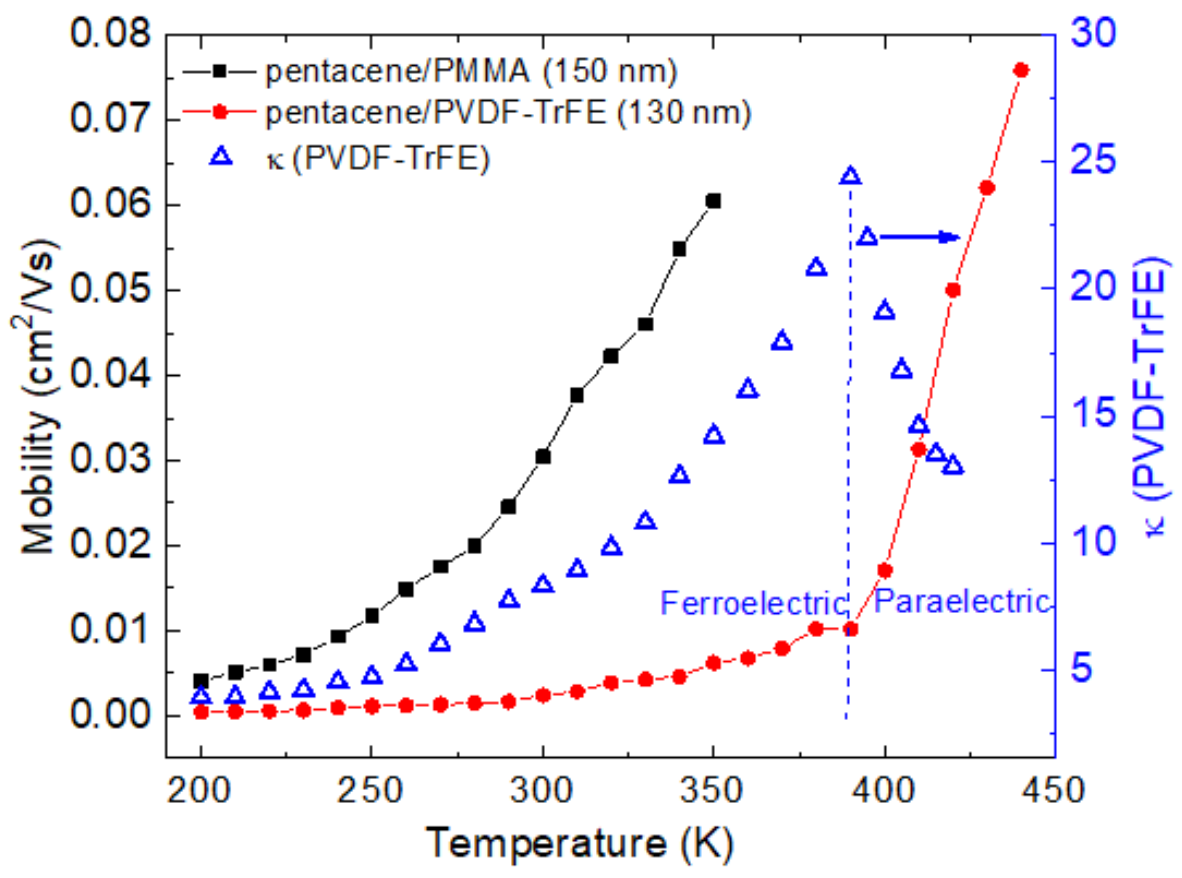

Figure 4.4 Comparison of the charge carrier mobility as a function of temperature in pentacene FETs using PMMA and PVDF-TrFE as dielectric layers. The dielectric constant of PVDF-TrFE as a function of temperature shown in the figure is from Ref. 42.

The dielectric constant of PVDF-TrFE as a function of temperature is plotted in figure 4.4 (Blue curve).$^{53}$ The discontinuity at $390 \mathrm{~K}$ denotes the change from the ferroelectric to the paraelectric phase. Figure 4.4 also shows the charge carrier mobility of pentacene FETs as a function of temperature with PMMA (150 nm thickness) and PVDF$\operatorname{TrFE}(130 \mathrm{~nm}$ thickness) as dielectric layers. All mobilities are extracted in the saturation region. We point out that both PMMA and PVDF-TrFE devices operate below $-4 \mathrm{~V}$. At room temperature, the charge carrier mobility of the PMMA-based FET in the saturation region $\left(0.03 \mathrm{~cm}^{2} / \mathrm{V} \mathrm{s}\right)$ is an order of magnitude lower compared to a prior work (where PMMA was dissolved in a high dipole moment solvent),${ }^{59}$ primarily due to the higher thickness of the dielectric layer in this work. 
Since PMMA degrades at higher temperatures, the pentacene/PMMA FET measurements were carried out only up to $350 \mathrm{~K}$. The PVDF-TrFE-based FETs were tracked all the way from the ferroelectric phase to above $\mathrm{T}_{\mathrm{c}}$. Agreeably, the charge carrier density may differ at the two dielectric interfaces but there are clear differences in the nature of carrier mobility versus temperature in the two devices. The PMMA device shows a sharp increase in its mobility with increasing temperature, whereas the PVDF-TrFE device shows a suppressed mobility in the ferroelectric phase, beyond which there is a large change in its mobility.

The suppression of the charge carrier mobility with increasing temperature in the ferroelectric phase may be attributed to a coupling of the charge carriers to the dielectric polarization of the gate insulator, resulting in a renormalization of the transfer integral, J. The dynamic coupling thus reduces $\mathbf{J}$ to $\mathrm{J}^{*}$, thereby increasing the effective mass $\left(\mathrm{m}^{*}\right)$ of the carriers. Theoretical calculations show that $\mathrm{m}^{*}$ increases by a factor of 1.27 in bulk pentacene due to molecular polarization effects. ${ }^{44}$ In order to understand whether there is a universality in the dynamical coupling due to the polarization effect and to distinguish between surface and bulk effects, we have carried out detailed temperature dependent current-voltage (I-V) measurements from pentacene FETs by varying the thickness of the PVDF-TrFE layer. These results are presented in the next section.

\subsection{Thickness-dependence of the ferroelectric layer}

The thickness of the PVDF-TrFE layer was varied from $20 \mathrm{~nm}$ to $500 \mathrm{~nm}$; the pentacene layer thickness was kept the same at $60 \mathrm{~nm}$ for all FETs. All devices show a similar trend for charge carrier mobilities versus temperature as shown in figure 4.4 , where 
$\mu_{\text {FET }}$ shows a sharp increase beyond the ferroelectric-paraelectric phase transition at 390 K. Figure 4.5 shows the Arrhenius plots of FET mobilities in the ferroelectric phase for varying thicknesses of the PVDF-TrFE layer. The $20 \mathrm{~nm}$ device (not shown here) shows an activation energy of $88 \mathrm{meV}$. We note that the activation energies $(\Delta)$ obtained by fits to Eq. (4.1), are much higher here than typically seen for pentacene FETs with nonferroelectric dielectrics. ${ }^{56}$ Furthermore, the correction due to thermalization of individual dipoles (as a result of the nonlinear contribution) when the system approaches Tc has not been taken into account here. ${ }^{53}$ Taking this correction may slightly reduce the mobility in the ferroelectric phase, thus further enhancing $\Delta$. Since the FETs in this work are low operating voltage devices, the nonlinear contribution may not be that high as evident from a prior work on polarization measurements from PVDF-TrFE capacitors with similar thicknesses. $^{60}$

The trend in Figure 4.4 clearly shows the activation energy to decrease with increasing thickness of the dielectric layer. This observation is consistent with slightly higher values of $\mu_{\mathrm{FET}}$ for thicker layers of PVDF-TrFE. In the ferroelectric region, there are thus two competing effects: activated hopping transport, which tends to increase $\mu_{\mathrm{FET}}$ with increasing temperatures, and the coupling of the carriers to the electric polarization in the form of surface Fröhlich polarons as $\mathrm{k}$ increases with temperature, which tends to decrease $\mu_{\text {FET }}$. The thinner dielectric layers induce stronger polarization fields, suppressing the charge carrier mobility even more compared to the thicker dielectric layers in the ferroelectric region. 


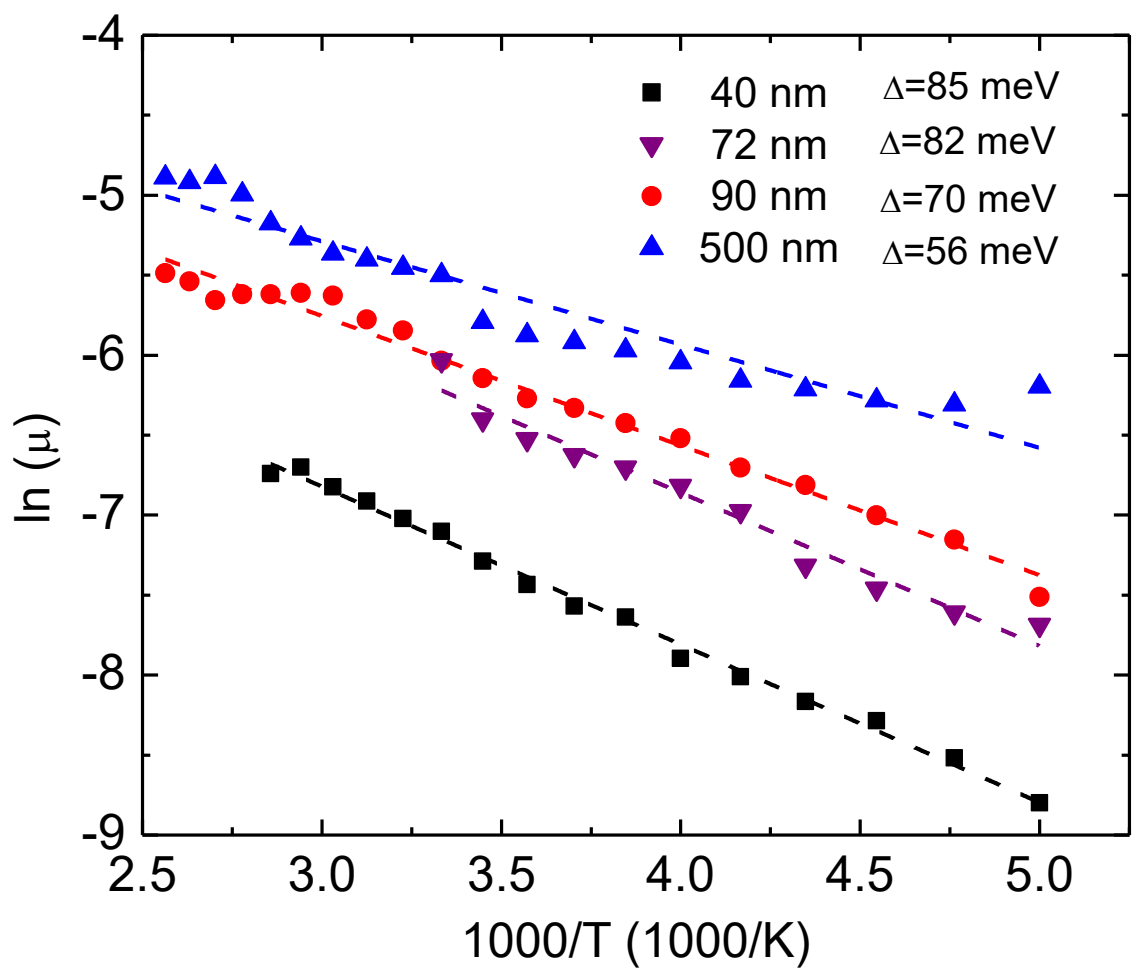

Figure 4.5 Arrhenius plots of FET mobilities in the ferroelectric phase for varying thicknesses of the PVDFTrFE layer.

The trapped charge density $\left(N_{\text {trap }}^{\max }\right)$, which is obtained from the subthreshold swing, as a function of temperature offers insights into the mechanism of transport in ferroelectric FETs. It is given by,

$$
N_{\text {trap }}^{\max } \approx\left[\frac{q \operatorname{SS} \log (e)}{k_{B} T}-1\right] \frac{C_{i}}{q}
$$

where $\mathrm{q}$ is the elementary charge and $\mathrm{C}_{\mathrm{i}}$ is the gate capacitance per unit area. The subthreshold swing, SS, is given by,

$$
S S=\left[\frac{d \log \left(I_{D S}\right)}{d V_{G S}}\right]^{-1}
$$


Equation (4.2) typically yields the maximum trapped charge. Using the change in $\mathrm{V}_{\mathrm{Th}}$ from I-V hysteresis measurements yields a lower limit of the trapped charge density. ${ }^{61}$ At room temperature, SS varies from $0.3 \mathrm{~V} / \mathrm{dec}$ to $2 \mathrm{~V} / \mathrm{dec}$ for the different thicknesses of the PVDF-TrFE layer. Figure 4.6 plots $N_{\text {trap }}^{\max }$ for three PVDF-TrFE devices as a function of temperature. Beyond $\mathrm{T}_{\mathrm{c}}$, there is a sharp decrease in the trapped charge density, which explains the large enhancement of $\mu$ in the paraelectric phase. The overall value of the trapped charge density decreases almost monotonically from $20 \mathrm{~nm}$ to $130 \mathrm{~nm}$, indicating that the surface states dominate in the thinner films.

(a)

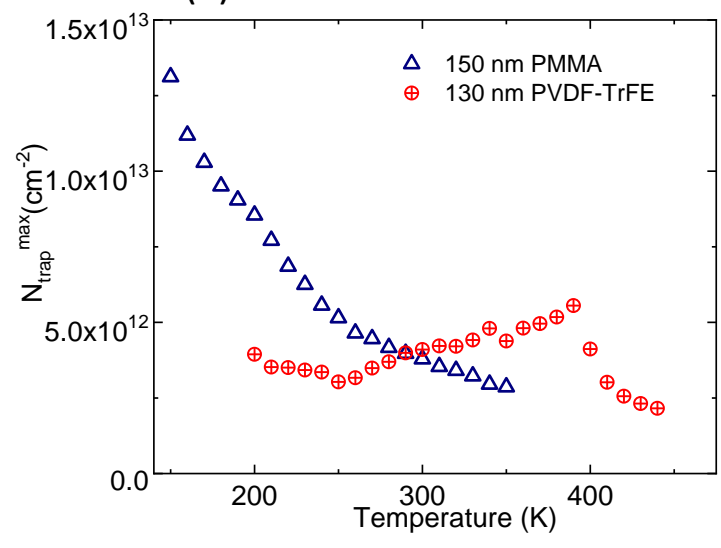

(b)

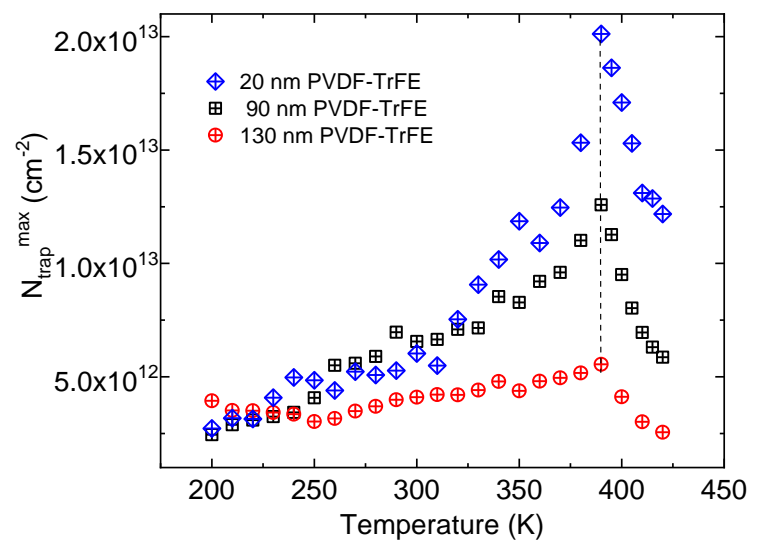

Figure 4.6 (a) Comparison of the trapped charge density of the PMMA-based pentacene OFET with a PVDF-TrFE-based OFET. (b) The trapped charge density as a function of temperature for the $20 \mathrm{~nm}, 90 \mathrm{~nm}$, and 130nm thick PVDF-TrFE devices.

Although the PMMA based device has a higher value of $N_{\text {trap }}^{\max }$ between $200 \mathrm{~K}$ and $300 \mathrm{~K}$, it shows a much higher value of $\mu_{\mathrm{FET}}$ compared to the PVDF-TrFE device in this temperature regime (Figure 4.6). These observations clearly show that the lower and the almost temperature-independent mobility in the ferroelectric phase of PVDF-TrFE FETs 
originate from a polarization fluctuation driven transport that dominates over the energetic disorder processes (observed in non-polar dielectrics).

\subsection{Stacked PVDF-TrFE/inorganic layers}

In order to distinguish the thermal contribution from the polarization contribution to the mobility $\left(\mu_{\mathrm{FET}}\right)$, stacked FETs were prepared where the PVDF-TrFE film was grown on $200 \mathrm{~nm}$ of $\mathrm{SiO}_{2}$. The $\mu_{\text {FET }}$ mobilities for three such pairs of devices are shown in figure 4.7, where the PVDF-TrFE film was identical for the single and stacked layers. In the stacked devices, the organic semiconductor-ferroelectric interface is still maintained, but since the gate electrode is now in contact with a non-ferroelectric dielectric, no effective change in polarization is expected. The stacked device is thus dominated by a "typical" temperature-dependent hopping transport, whereas the single ferroelectric-based FET is dominated by polarization fluctuations in the ferroelectric phase (below $\mathrm{T}_{\mathrm{c}}$ ).
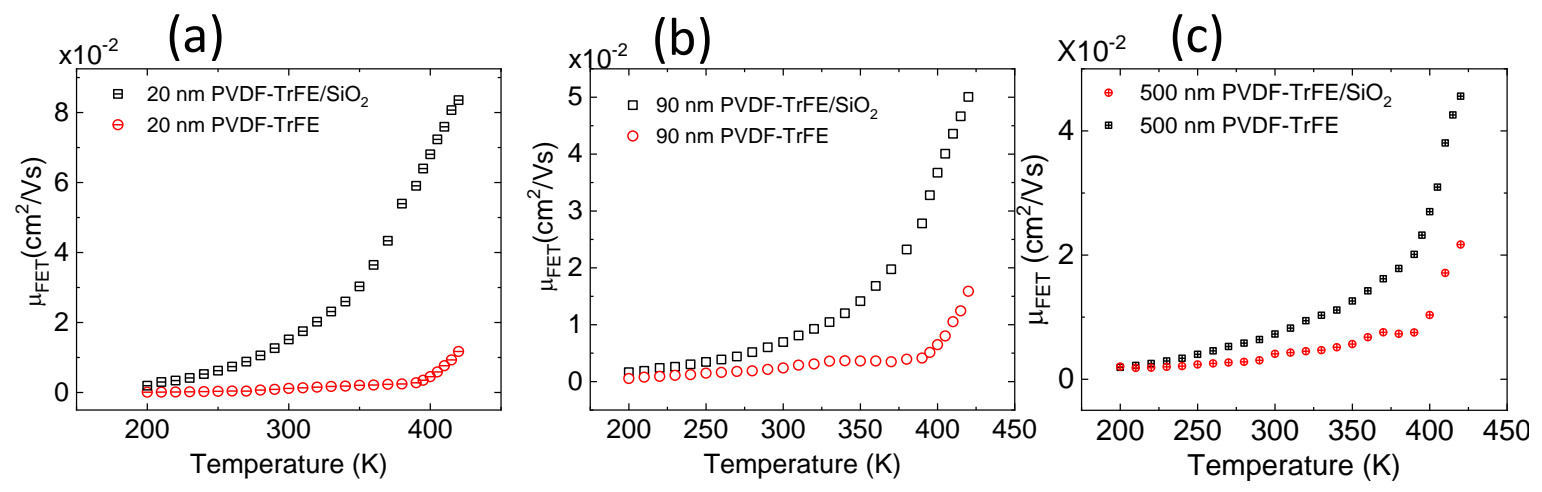

Figure 4.7 Comparison of single layer PVDF-TrFE and stacked PVDF-TrFE/ $\mathrm{SiO}_{2}$ OFETs with (a) $20 \mathrm{~nm}$, (b) $90 \mathrm{~nm}$, and (c) $500 \mathrm{~nm}$, of the PVDF-TrFE thickness. 
Assuming that the ferroelectric component of the stacked device contributes negligibly to transport, the mobility $\left(\mu_{\text {stack }}\right)$ as a function of temperature may be used as a reference to take into account the contribution from all additional factors. By using the Matthiessen's rule,

$$
\mu_{F E T}=\left[\frac{1}{\mu_{P}}+\frac{1}{\mu_{\text {stack }}}\right]^{-1}
$$

the contribution due to the Fröhlich polarons, $\mu_{\mathrm{P}}$, may be extracted from the knowledge of the temperature dependence of the mobility of single layer ferroelectric and stacked devices. The strongly coupled Fröhlich polaron mode ${ }^{27}$ yields,

$$
\mu_{P}(T)=\frac{e a^{2} \omega_{S}}{8 k_{B} T} \exp \left(-\frac{\Delta_{P}}{k_{B} T}\right)
$$

where $\omega_{\mathrm{S}}$ is the frequency of the surface phonons of the dielectric, $a$ is the hopping length, and $\Delta_{\mathrm{P}}$ is the polaron hopping barrier. We note that $\omega_{\mathrm{S}}$ is related to the longitudinal $\left(\omega_{\mathrm{L}}\right)$ and transverse $\left(\omega_{\mathrm{T}}\right)$ optical phonon frequencies by,

$$
\omega_{S}^{2}=\frac{1}{2}\left(\omega_{L}^{2}-\omega_{T}^{2}\right)
$$

The factor of 8 in the denominator of Eq. 4.5 results from considering an upper bound to the mobility in the hopping process. The Einstein's relation for a nondegenerate carrier gas yields $\mu=\frac{q D}{k k_{B} T}$, where $\mathrm{D}$, the diffusion coefficient, is given by $\gamma \mathrm{a}^{2} / 4$ in two-dimensional hopping motion, and $\gamma$ is the hopping rate. The probability of hopping takes on a factor of 1/2 for each attempt to pass the barrier in the forward direction. Figure 4.8 shows the temperature-dependent mobility of Fröhlich polarons for FETs with $500 \mathrm{~nm}, 90 \mathrm{~nm}$, and 
$40 \mathrm{~nm}$ thick PVDF-TrFE layers, which were extracted by comparing the single layer devices with the stacked layers as explained above. The experimental data in the ferroelectric phase were fit with Eq. (4.5), and the parameters are tabulated in Table 4-I. For clarity, we do not include the $20 \mathrm{~nm}$ data in figure 4.8 . We use $\omega_{\mathrm{s}}=500 \mathrm{~cm}^{-1}$ in the fits, which is reasonable since the $\beta$ phase of PVDF is characterized by $\omega_{\mathrm{L}}$ and $\omega_{\mathrm{T}}$ at $\approx 500 \mathrm{~cm}^{-}$ 1. ${ }^{62,63}$ The hopping length increases with the thickness of the PVDF-TrFE layer, consistent with the slightly larger mobilities observed for the thicker dielectric devices. For single crystals such as rubrene, which show at least two orders of magnitude higher FET mobility compared to this work, the hopping length is theoretically found to be greater than $7 \AA .^{44}$ One can further estimate the polaron binding energy from the polaron hopping barrier. The hopping barrier is related to the binding energy, $E_{\mathrm{P}}$, by

$$
\Delta_{P} \approx \frac{1}{2} E_{P}-J *
$$

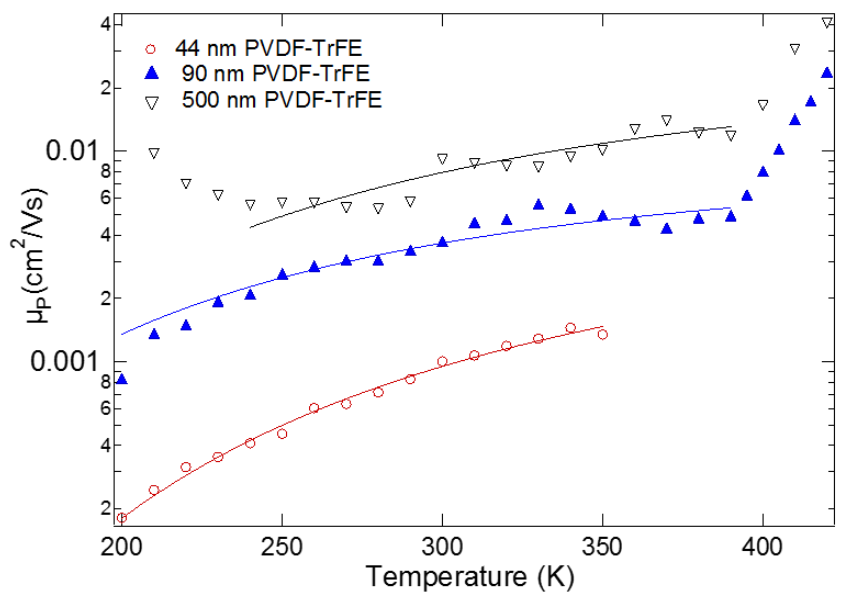

Figure 4.8 Temperature-dependent mobility of Fröhlich polarons for pentacene FETs with three thicknesses of the PVDF-TrFE layer. The bold line is a fit to the model discussed in the text. 
Table 4-I The polaron hopping barrier and hopping lengths obtained by fits to Eq. (4.5).

\begin{tabular}{lll}
\hline PVDF-TrFE thickness $(n m)$ & $\Delta_{\mathrm{P}}(\mathrm{eV})$ & $\mathrm{a}(\AA)$
\end{tabular}

\begin{tabular}{lll}
\hline 500 & $0.085 \pm 0.007$ & $5.55 \pm 0.70$ \\
90 & $0.078 \pm 0.005$ & $2.90 \pm 0.30$ \\
40 & $0.107 \pm 0.004$ & $2.85 \pm 0.20$ \\
20 & $0.096 \pm 0.009$ & $2.37 \pm 0.40$ \\
\hline
\end{tabular}

In equation 4.7 , using the value of $\mathrm{J}^{*}=44.9 \mathrm{meV}$ for a pentacene $/ \mathrm{Ta}_{2} \mathrm{O}_{5}$ interface from Ref. $^{44}$ similar to our device interface, $\mathrm{E}_{\mathrm{P}} \approx 260 \mathrm{meV}$ was obtained for the $500 \mathrm{~nm}$ device, suggesting that the strongly coupled polaron model is appropriate to describe transport for polycrystalline pentacene FETs with a ferroelectric dielectric. Such a moderately high binding energy we observed also explains the low and almost temperature-independent $\mu_{\mathrm{FET}}$ in the ferroelectric phase. For a rubrene/ $\mathrm{Ta}_{2} \mathrm{O}_{5}$ interface, the polaron binding energy was theoretically obtained as $\approx 100 \mathrm{meV}$.

\subsection{Raman scattering from pentacene on PVDF-TrFE}

One of the questions that arise is whether the change in the transport properties of pentacene FETs on PVDF-TrFE is related to an electron-phonon coupling mechanism originating from the semiconductor layer itself. Reports on the phase transition of PVDF and its copolymers have been followed by Raman scattering. ${ }^{64}$ Figure 4.9 shows the Raman spectra of pentacene from 1100 to $1700 \mathrm{~cm}^{-1}$ from room temperature to above Tc of the dielectric layer. The aromatic stretch vibration lies in the $1340-1400 \mathrm{~cm}^{-1}$ range. The two 
peaks at 1160 and $1178 \mathrm{~cm}^{-1}$ are related to a $\mathrm{C}-\mathrm{H}$ bending motion. The $1371 \mathrm{~cm}^{-1}$ peak is seen as the strongest C-C stretch vibration. Details of the Raman spectrum of pentacene are found in Ref. ${ }^{65}$ As such, there are no abrupt changes in the Raman phonon frequencies or line shapes of pentacene as the underlying PVDF-TrFE layer undergoes a phase transition. A softening of the Raman frequencies with increasing temperature is observed, which is typical for any solid. Furthermore, we have also compared the Raman spectrum of pentacene on a non-polar dielectric layer with similar temperature sweeps as on PVDFTrFE; the slight changes in the frequencies and broadening of the Raman peaks with temperature are identical on both interfaces. These results conclusively show that changes in the transport properties arise from a coupling of the carriers with the dielectric interface.

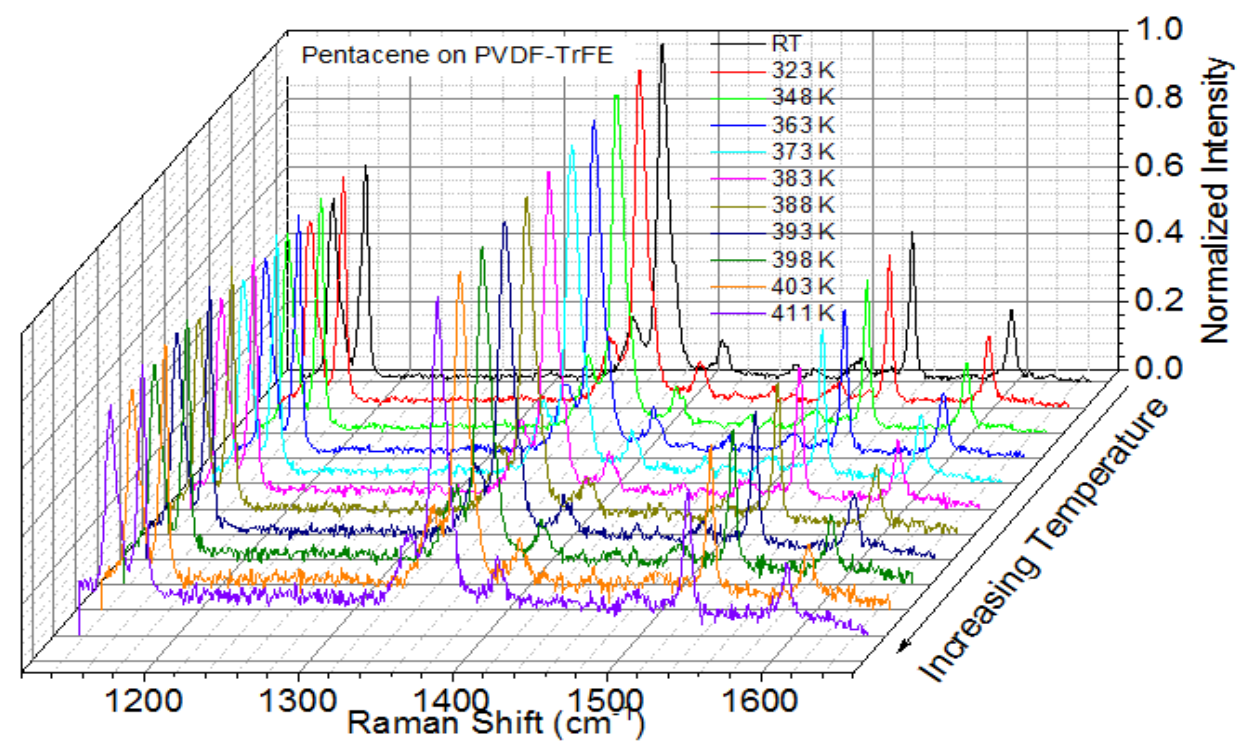

Figure 4.9 Raman spectra of pentacene on PVDF-TrFE as a function of temperature.

As a summary, the charge transport in ferroelectric-based FETs is strongly influenced by a polarization dominant transport rather than an energetic disorder within the 
framework of a hopping transport. The ferroelectric dielectric, PVDF-TrFE, which shows a large change in its dielectric constant as the temperature is swept from $200 \mathrm{~K}$ to the ferroelectric paraelectric phase transition temperature at $390 \mathrm{~K}$, provides a testbed to study interfacial transport as the polarization strength is tuned. When the gate insulator is sufficiently polar, Fröhlich polarons play an important role in interfacial transport. Temperature-dependent charge carrier mobility in pentacene FETs shows almost no change as long as the dielectric layer is in the ferroelectric phase due to the coupling of the charge carriers with the surface phonons of the dielectric layer. This effect is more pronounced for thinner dielectric films. The threshold voltage and the trapped charge density also show a discontinuity at the ferroelectric paraelectric phase transition. To extract the polarization contribution to the temperature dependence of the charge carrier mobility from mechanisms other than Fröhlich polarons, ideally one should look at a pentacene FET where the gate insulator is vacuum. This is clearly not an option for a polycrystalline film such as pentacene. The bilayer $\left(\mathrm{SiO}_{2} / \mathrm{PVDF}-\mathrm{TrFE}\right)$ devices show a typical temperature-dependent mobility as seen with non-polar dielectrics. Temperaturedependent mobility was extracted by comparing the bilayer devices with single layer PVDF-TrFE pentacene FETs. Using a strongly coupled Fröhlich polaron model, we estimate the hopping length and the hopping barrier of the polarons. The hopping lengths vary between $2 \AA$ and $5 \AA$. 


\section{Transport properties in TIPS-pentacene FETs and diodes}

In chapter 4 we saw that the presence of PVDF-TrFE as a gate dielectric influences the charge transport in pentacene FETs via formation of the Fröhlich polarons. One question that arises is: whether polarization fluctuation inherent to PVDF-TrFE influences transport in other organic FETs? This chapter seeks to understand this question. By focusing on TIPS-pentacene as the organic semiconductor we investigate the role of polarization fluctuation in FET transport.

\subsection{Charge transport models in disordered systems}

We touched briefly on charge transport mechanisms in organic semiconductors in chapter 2. Here we describe charge transport models in disordered systems in more detail. Disordered systems are characterized both by extended and localized states; transport typically occurs through the exponential tail states. Figure 5.1 shows a schematic of the density of states (DOS) for a crystalline and an amorphous semiconductor. In the absence of a long-range order, as in amorphous materials, the sharp features in the DOS disappear. The concept of a mobility edge is often invoked in disordered systems to discuss transport; it plays the same role as band edges in a crystal. ${ }^{66}$ In amorphous semiconductors, disorderinduced DOS bandtails are present up to their mobility edge in addition to localized defect states near the Fermi energy $\left(\mathrm{E}_{\mathrm{F}}\right)$. If the Fermi level is located in the region of localized states as depicted in figure 5.1 (a), the conductivity may arise either from thermal excitation of carriers across a mobility gap into extended states or by a hopping process. The former mechanism is the dominant process at high temperatures, and the latter dominates charge transport at lower temperatures. The concept of transport energy (TE) has been developed 
to describe hopping transport in band tails with purely exponential DOS. The DOS may be described by,

$$
g(E)=\frac{N_{0}}{E_{0}} \exp \left(-\frac{E}{E_{0}}\right)
$$

where $N_{0}$ is the concentration of localized tail states, and $E_{0}$ is the tailing parameter such that $E_{F}$ is thought to be deep in the gap. The localization energy, $E$, is measured positive from the mobility edge $(\mathrm{E}=0)$ towards the gap center. By comparing the thermal energy to $E_{0}$, one may have different regimes in conductivity. At high temperatures, conductivity is due to activation of carriers from the Fermi level into extended states. At low temperatures, Mott's law for variable range hopping is applicable and at intermediate temperatures, conductivity may still be determined by variable range hopping but the exponential growth of DOS results in a so-called transport energy.

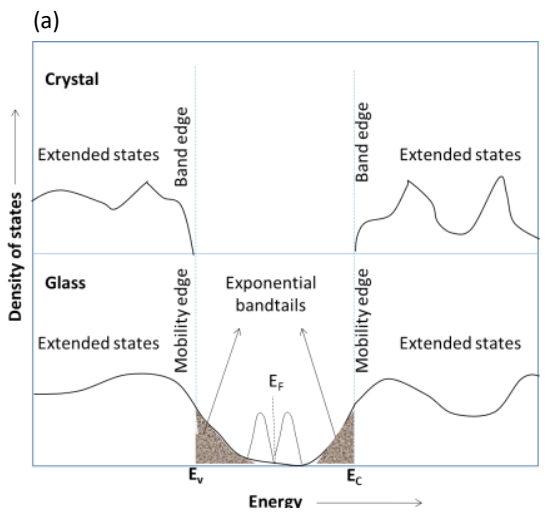

(b)

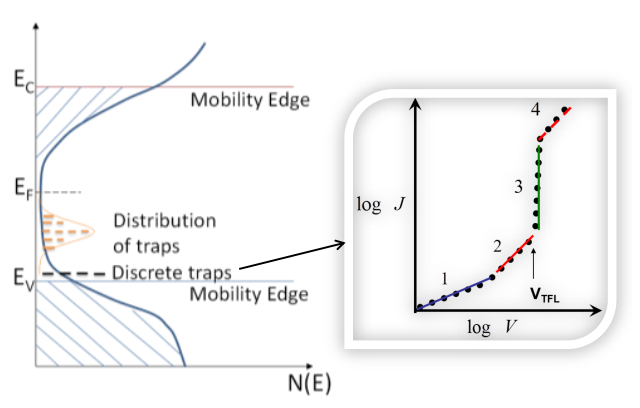

Figure 5.1 (a) Schematic of DOS for a crystalline and an amorphous semiconductor. The mobility edges in an amorphous semiconductor play a similar role as the band edges in a crystal. (b) Schematic of trap states. (c) Schematic of discrete trap space-charge-limited conduction in a log-log plot of current density (J) versus voltage. 
The concept of transport energy was introduced by Monroe in terms of a nonequilibrium energy relaxation problem where the electrons directly hop between localized, bandtail states. ${ }^{58}$ Starting from the mobility edge, the electrons hop downward in energy. This relaxation process changes at some particular energy represented as the transport energy (TE). The hopping process near and below this energy resembles dispersive transport with TE playing the same role as the mobility edge.

The hopping rate of electrons between localized states may be described by Miller Abraham's formalism ${ }^{67}$,

$$
v_{i j}=v_{0} \exp \left(-2 \frac{R_{i j}}{\alpha}-\frac{\left(E_{i}-E_{j}+\left|E_{j}-E_{i}\right|\right)}{k_{B} T}\right)
$$

In Eq. (5.2), $v_{i j}$ is the rate of hopping between an occupied site $i$ (with energy $E_{i}$ ) and an empty state $j$ (with energy $E_{j}$ ), separated by a distance $R_{i j} ; \alpha$ is the decay length of the wave function; $v_{0}$ is the attempt-to-hop or attempt-to-escape frequency. Since the decreasing DOS makes it difficult for a charge carrier to find a neighbor at low energies, carriers in deep states move by thermal excitation to shallower states, whilst carriers in shallow states move by hopping to other shallow states.

Organic and polymeric semiconductors are usually characterized by a Gaussian DOS rather than the exponential bandtail discussed earlier. The rationale is that the distribution of disordered potential leads to electron localization, which is Gaussian. Although one can invoke the concept of TE with Gaussian DOS, an exact analytical form is not possible. Furthermore, the concept of TE in organic semiconductors provides a reasonable description of variable range hopping, where transport is via hopping in the 
band tails. One of the implications of the Gaussian DOS is that carriers have a finite mobility:

$$
\mu=\mu_{0} \exp \left(-\left(\frac{2 \sigma}{3 k_{B} T}\right)^{2}\right)
$$

where $\sigma$ is the width of the Gaussian DOS. Phenomenologically, the temperature and field dependence of carrier mobility in disordered polymers is often modeled as: ${ }^{68}$

$$
\mu(T, E)=\mu_{0} \exp \left[-\left(\frac{2 \sigma}{k_{B} T}\right)^{2}\right] \times \exp \left[\left[C\left(\frac{\sigma}{k_{B} T}\right)^{2}-\Sigma^{2}\right] E^{1 / 2}\right]
$$

where $C$ is an empirical constant, and $\Sigma$ is a parameter that may be related to Miller Abraham's formalism (Eq. 5.4), denoting the local variations of the nearest-neighbor intersite distance.

Inorganic semiconductors, which are described in terms of delocalized electronic bands, show a very different mechanism of carrier transport. Here the carriers move as a highly delocalized plane wave. In ordered organic semiconductors, the temperature dependence of the carrier mobility is more band-like with $\mu=\mu_{0} T^{-n}$, where $n$ lies between 1 and 2. For these systems there is almost no field dependence of $\mu$. Thus, the absence of field-dependent mobility in organic transistors is tell-tale of a band-like transport.

The description of transport in organic thin films is further complicated by the presence of traps. These trap states could arise from chemical impurities, structural defects, or be due to grain boundaries in organic polycrystalline films. They could be shallow or deep traps. In the following section, we briefly discuss trap states, mainly discrete traps, and how they can be identified in bulk transport behavior. 


\subsubsection{Space-charge-limited-conduction (SCLC) and shallow traps}

SCLC based models have been used to reveal the nature of trap states in organic molecules and polymers for over 50 years. Typically, most amorphous polymers are characterized by a Gaussian distribution of traps; however, in crystalline films of naphthalene and anthracene, shallow trapping with a single trap level was observed. ${ }^{69}$ Subsequently, discrete traps have also been observed in a class of semicrystalline polyfluorenes. ${ }^{70} \mathrm{~A}$ schematic of these trap states is shown in figure 5.1 (b) where a contribution of both discrete traps close to the mobility edge and a Gaussian distribution are shown. Current-voltage measurements from two-terminal organic semiconductorbased devices with good Ohmic contacts reveal the nature of bulk traps.

Discrete trap SCLC is typically characterized by four regions in a log-log plot of the current-density $(J)$ versus voltage $(V)$ as schematically shown in figure 5.1 (c). The first is the Ohmic region with $J=\left(q n_{0} \mu V\right) / d$, where $q$ is the charge, $n_{0}$ is the free carrier density, $d$ is the thickness of the film between the electrodes, and $\mu$ is the carrier mobility. The second region is trap-limited SCLC with,

$$
J=\frac{9}{8} \varepsilon \varepsilon_{0} \mu \theta \frac{V^{2}}{d^{3}}
$$

which is similar to Child's law seen in vacuum diodes. In Eq. (5.5), $\theta$ (the trapping fraction) is related to the trapping energy $\left(E_{t}\right)$ by, $\theta \propto \exp \left(-E_{t} / k_{B} T\right), \varepsilon$ is the dielectric constant of the polymer, and $\varepsilon_{0}$ is the permittivity of free space. For an exponential distribution of traps, $J \propto V^{m}$, where $m>2$. Region 3 in figure 5.3 (c) which shows a vertical rise in current, is the trap-filled limit. Here the onset of the trap-filled voltage $\left(V_{T F L}\right)$ is related to the 
density of traps $\left(N_{t}\right)$ by, $N_{t}=\frac{3}{2} \frac{\varepsilon \varepsilon_{0} V_{T F L}}{q d^{2}}$. The fourth region depicts current limited by the space charge and is free from the influence of traps. This region is governed by the same expression as region 2 (Eq. (5.5)), except that $\theta=1$. Therefore, if all four regions are observed as schematically shown in figure 5.1 (c), the carrier mobility and other trap related parameters can be easily determined.

\subsection{Transport in two terminal devices}

Charge transport in three terminal FET devices where the dielectric plays an important role, has been discussed in chapter 4 . The nature of traps plays a significant role in dictating charge transport. Temperature-dependent current density versus voltage $(\mathrm{J}-\mathrm{V})$ measurements from two terminal devices allow an independent measure of transport in organic semiconductors. J-V characteristics were carried out from two-terminal devices of pentacene. Since pentacene is uniformly deposited by evaporation, we used a sandwiched structure (ITO/pentacene/Au) for measuring the $\mathrm{J}-\mathrm{V}$ characteristics. As seen in figure 5.2, the J-V curve in pentacene does not signal discrete trap SCLC as all four regions described in previous section (5.1.1) are not observed. The trap-limited region agrees with $J \propto V^{2}$. We thus use Eq. (5.5) to obtain bulk $\mu$ at all temperatures. Since the trapping fraction is unknown, we obtain the product of $\mu \theta$ as a function of temperature. The inset of figure 5.2 (a) clearly shows that the slope of the J-V curve increases with temperature. In the temperature range used for these measurements, one does not expect $\theta$ to change much. From figure 5.2 (b), we thus conclude that the bulk carrier mobility in pentacene increases with temperature, which is a clear signature of an activated transport. This result lines up with what we saw in FET transport (figure 4.3). 

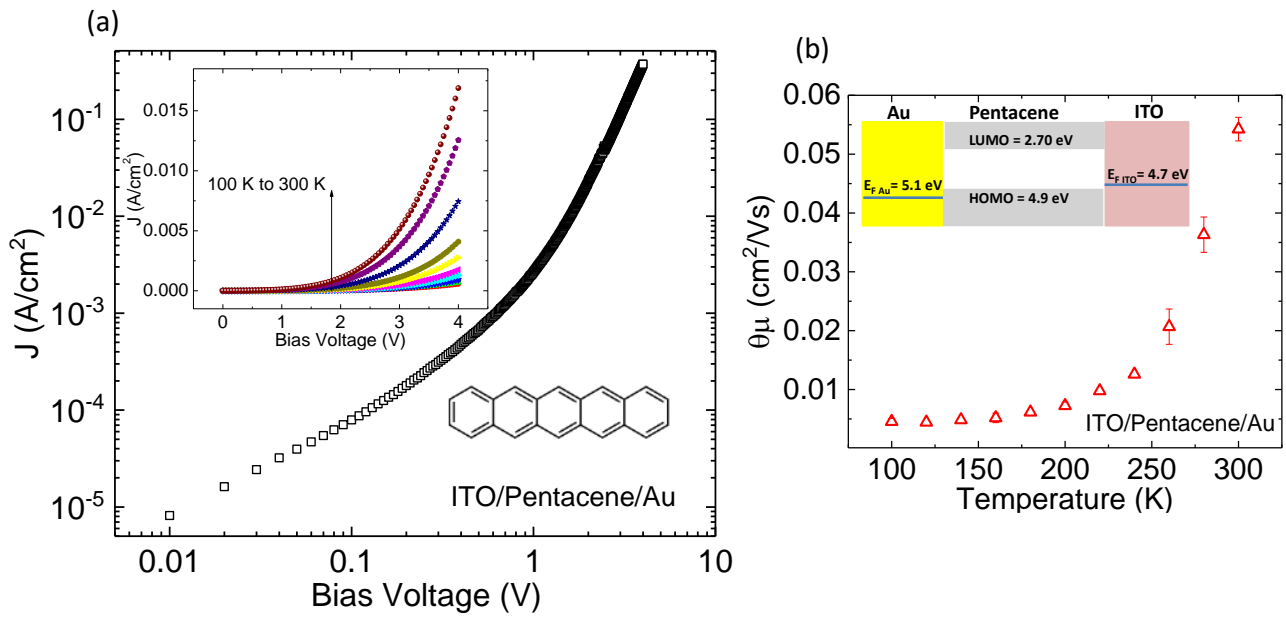

Figure 5.2 (a) J-V characteristics (log-log plot) of pentacene at $300 \mathrm{~K}$. The inset shows the J-V behavior at different temperatures. The chemical structure of a pentacene molecule is shown in the inset. (b) Product of the trapping fraction and carrier mobility as a function of temperature for a pentacene diode. (Inset) Schematic of the energy levels.

We also want to monitor the J-V characteristics in other semiconductors. We use another small molecule solution processable semiconductor TIPS-pentacene. In the case of TIPS-pentacene we use the two Au terminals across the organic semiconductor layer for measuring the $\mathrm{J}-\mathrm{V}$ characteristics as a function of temperature. The orientation of the crystalline growth in TIPS-pentacene is along the two terminals.

Figure 5.3 (d) shows the J-V data of TIPS-pentacene two terminal device at $300 \mathrm{~K}$ in a log-log plot. Transport in TIPS-pentacene is quite different than in pentacene. One clearly sees the signature of discrete traps with all four regions as discussed in section (5.1.1). Since trap-free SCLC is observed, one can use the modified version of Eq. (5.5) where $\theta=1$ to extract $\mu$. The temperature-dependence $\mathrm{J}-\mathrm{V}$ curves are shown in figure 5.3 (b) and (c) in non-log plots. Beyond $180 \mathrm{~K}$, the slope of the curves decreases (red arrow). Interestingly, the J-V characteristics from TIPS-pentacene diodes signal a SCLC for a 
discrete set of trap levels, suggesting that charge injection and transport occur through regions of ordering in the semiconductor. By using the same region for the trap-free SCLC at all temperatures, the bulk carrier mobility ( $\mu_{\text {bulk }}$ ) is extracted, as plotted in the inset of figure 5.3 (d). A clear decrease in $\mu_{\text {bulk }}$ is observed beyond $180 \mathrm{~K}$.

(a)

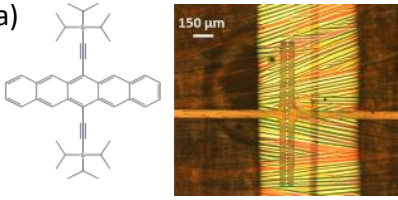

(b)
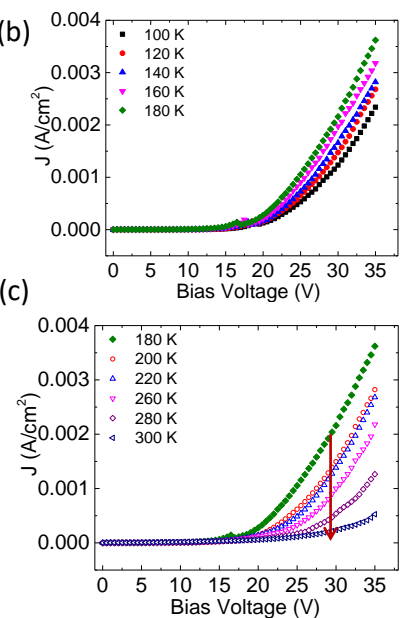

(d)

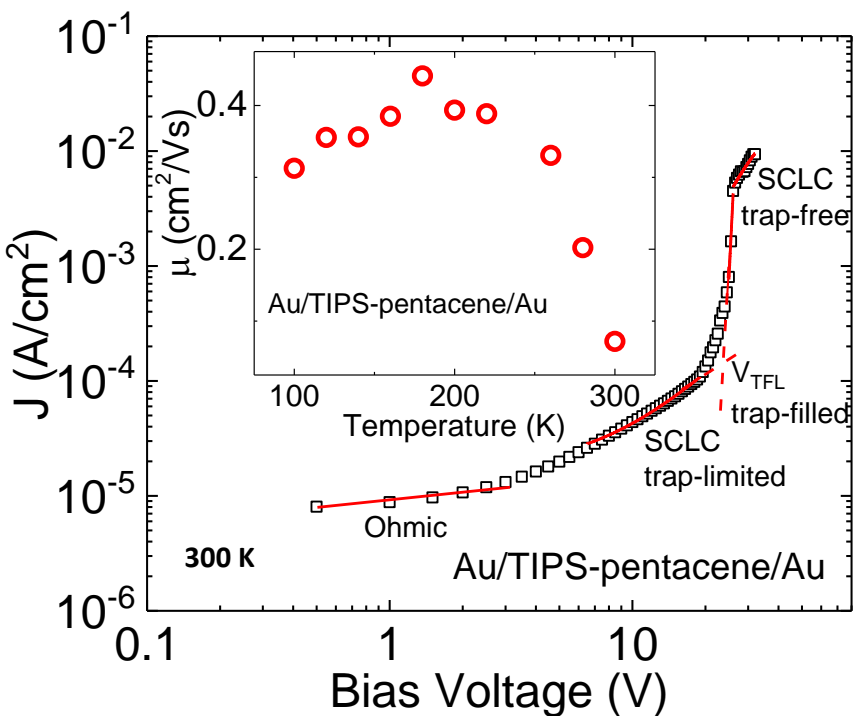

Figure 5.3 (a) Chemical structure of TIPS-pentacene and an optical image from a TIPS-pentacene device.

(b) and (c) J-V curves from a TIPS-pentacene two-terminal device as a function of temperature. (d) J-V curve (log-log plot) at $300 \mathrm{~K}$ showing all four regions, characteristics of discrete trap SCLC. Inset shows the bulk carrier mobilities as a function of temperature.

In comparing pentacene to TIPS-pentacene, we found that the $\mathrm{J}-\mathrm{V}$ diode characteristics are quite different: TIPS-pentacene, which shows discrete trap SCLC, yields a decreasing mobility beyond a certain temperature. The origin of the shallow discrete traps in TIPS-pentacene could be structural or due to other chemical defects and may occur at grain boundaries. Pentacene, on the other hand, which is characterized by a distribution of 
traps, yields an activated transport with increasing bulk $\mu$ as a function of increasing temperature, in agreement with FET transport as discussed in chapter 4.

\subsection{Role of dielectrics in charge transport}

The field of organic FETs has seen an increasing usage in polymer dielectric materials and a progressively lower dependence on traditional oxide dielectrics, such as $\mathrm{SiO}_{2}$ and $\mathrm{Al}_{2} \mathrm{O}_{3}$. Much effort has gone into modification of the dielectric interface; appropriate gate dielectrics that are free of electron-trapping groups, such as hydroxyl or silanol, have allowed the demonstration of n-channel FETs. ${ }^{71}$ In order to achieve stable and low-operating voltage FETs, the dielectric surface should be hydrophobic and must provide a relatively high capacitance, which is often a challenge with polymer dielectrics as they have an inherently low value of $\kappa$. However, using techniques for lowering the thickness of polymer dielectrics, high capacitance may be achieved.

As discussed in chapter 4, Fröhlich polarons are observed in molecular semiconductor based FETs when the gate dielectric is sufficiently polar. ${ }^{51}$ A consequence of such an interaction would be lower carrier mobility. Thus, one would expect that as $\kappa$ of the dielectric medium increases, the FET carrier mobility should decrease. The polarization field of a ferroelectric dielectric should have an additional contribution to the carrier mobility. As an example, we plot the FET carrier mobilities of TIPS-pentacene FETs at $300 \mathrm{~K}$ for various polymer and inorganic dielectrics used in our study (Figure 5.4). Figure 5.4 (a-c) shows the various FET geometries used in our work, where either a top or a bottom gate dielectric geometry has been used. CYTOP $(\kappa=2)$ is extremely hydrophobic; hence it is easier to use it as top gate dielectric rather than a bottom gate dielectric. We will later 
discuss the advantages of the dual gate structure (figure 5.4 (b)). Figure 5.4(d) clearly shows that with increasing $\kappa$ of gate dielectrics, the carrier mobility decreases. Although poly(4-vinylphenol) (PVP) has a similar $\kappa$ value to that of $\mathrm{SiO}_{2}$, we obtain a slightly lower value for carrier mobility with $\mathrm{SiO}_{2}$, which is not surprising since oxide dielectrics are notorious for trapping charges at the interface. PVDF-TrFE and $\mathrm{Al}_{2} \mathrm{O}_{3}$, which have the highest $\kappa$ values at $300 \mathrm{~K}$, show the lowest carrier mobility. Additionally, the polarization field of PVDF-TrFE plays a role in carrier transport, as will be elaborated in coming sections.

(a)

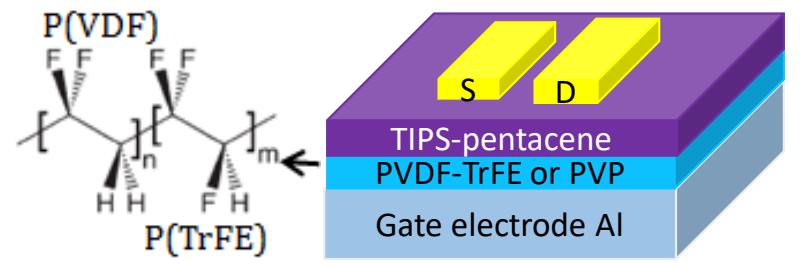

(b)

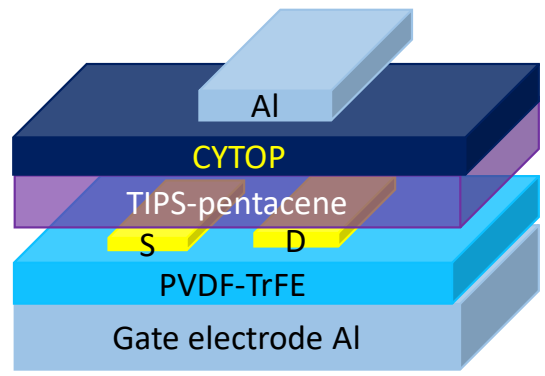

(c)

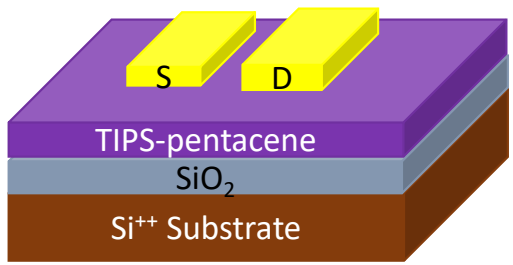

(d)

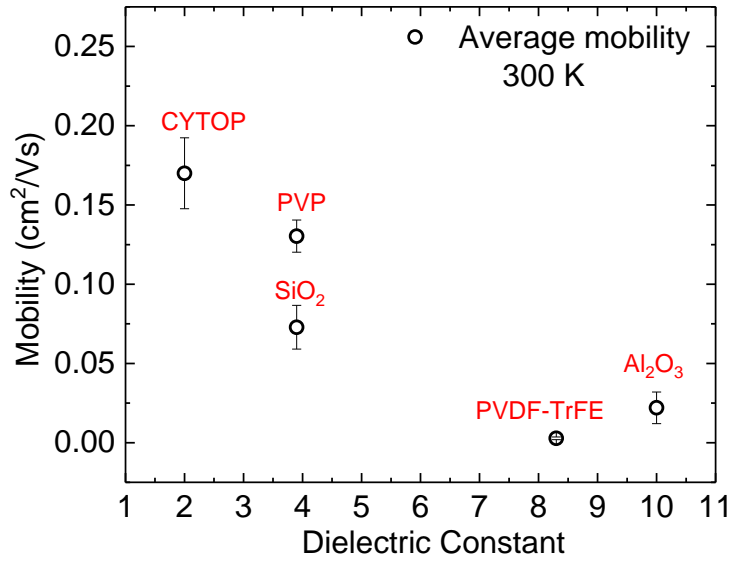

Figure 5.4 Schematic of FET structures. (a) Bottom gate PVDF-TrFE (chemical structure shown) or PVP (b) dual FET with PVDF-TrFE as bottom gate and CYTOP as top gate (c) bottom gate $\mathrm{SiO}_{2}$ (d) Average carrier mobility of TIPS-pentacene for different gate dielectrics. 


\subsection{Carrier transport in TIPS-pentacene FETs}

In section 5.2 we saw that the bulk transport in pentacene and TIPS-pentacene are quite different. Furthermore, TIPS-pentacene shows an unusual property similar to single crystals, where the mobility decreases with increase in temperature, which led us to investigate the temperature dependent transport in TIPS-pentacene.

In single-crystal organic FETs such as rubrene, the disorder is significantly reduced, enabling the realization of intrinsic polaronic transport, including the observation of the Hall effect in the accumulation layer. ${ }^{72}$ The Hall voltage is a sensitive probe that measures the extent of delocalized adiabatic transport. The presence of the normal Hall effect in organic FETs is, therefore, a signature that the surface charge is similar to a twodimensional hole or an electron-gas system rather than a hopping of carriers. ${ }^{73}$

There have been several recent works that show band-like transport, characterized by a negative coefficient of the charge-carrier $\mu$ with respect to temperature $(T)(d \mu / d T<$ 0), in solution-processable small-molecule and polymer-based FETs. In TIPS-pentacene a negative coefficient of the carrier mobility at high electric fields was attributed to localized transport limited by thermal-lattice fluctuation. ${ }^{12}$ In a few acene-based solutionprocessable small molecule FETs, charge delocalization induced by the dynamic disorder as a function of temperature has been probed by charge-modulation spectroscopy, shedding light on the nature of shallow traps. ${ }^{74}$ By incorporating large thermal-lattice fluctuations with weak van der Waals intermolecular bonding, theoretical models have explained the band-like motion observed at high temperatures from highly conducting organic

semiconductors. ${ }^{75}$ In such materials, the carriers diffuse incoherently in the presence of 
the large thermal fluctuations, resulting in a weakly metallic behavior. Band-like transport in TIPS-pentacene FETs has also been observed by time-resolved optical secondharmonic-generation imaging. ${ }^{76} \mathrm{~A}$ common feature for $\mathrm{d} \mu / \mathrm{dT}<0$ to occur beyond a certain temperature, as seen across a variety of organic semiconductors, is that the FET carrier mobility should be larger than $\mu_{\min }=\mathrm{er}^{2} / 2 \hbar$, where $\mathrm{r}$ is the intermolecular distance, e is the electronic charge, and $\hbar$ is the Planck's constant. ${ }^{77}$ Typically, this condition translates to FET mobilities being greater than 1 to $2 \mathrm{~cm}^{2} / \mathrm{Vs}$, ensuring that the energy bandwidth is larger than the energy change involved in the scattering. Recently, ac Hall Effect measurements have shed light on the coexistence of both band-like and hopping charge carriers in the accumulation region of organic FETs. Because of this mixed transport regime, even when FET carrier mobilities are less than $1 \mathrm{~cm}^{2} / \mathrm{Vs}$, a Hall voltage is measurable. ${ }^{78,79}$ It may be prudent to point out that band-like transport in molecular semiconductors, which mainly occurs in narrow bands, is somewhat different from true band transport, where the latter occurs through extended states in perfect crystals.

In pure organic crystals such as naphthalene, band transport for electrons was observed below $150 \mathrm{~K}{ }^{80}$ Subsequently, first-principles calculations involving inter- and intramolecular vibrations and electron-phonon coupling constants (both local and nonlocal) have elucidated band-to-hopping transition in naphthalene as a function of temperature. ${ }^{81}$ This transition can be understood on the basis of localization of charge carriers in the absence of any thermal disorder. Furthermore, at the transition where the charges are more delocalized, raising the temperature destroys the translational symmetry and reduces the charge transport efficiency, and thus its mobility. ${ }^{82}$ The apparent band-like transport observed as $\mathrm{d} \mu / \mathrm{dT}<0$ in organic and polymeric FETs may be mediated by 
extrinsic mechanisms. As we see here, depending on the nature of the dielectric used, a negative or a positive coefficient of $\mu$ with respect to temperature is observed in TIPSpentacene FETs, and this behavior is further correlated to the nature of the shallow traps. We explore the influence of both ferroelectric and nonferroelectric dielectrics on the transport mechanism in TIPS-pentacene FETs using top-gate and bottom-gate architectures. Unlike nonferroelectric dielectrics, the temperature-dependent mobility when using PVDF-TrFE shows a negative coefficient beyond $200 \mathrm{~K}$, similar to the trend observed in diodes without the influence of dielectric.

\subsubsection{Temperature-dependent FET characteristics with non-ferroelectric dielectrics}

We first look at the trends in carrier mobilities and other transistor parameters in TIPS-pentacene FETs using non-ferroelectric dielectrics: $\mathrm{Al}_{2} \mathrm{O}_{3}$, CYTOP, PVP and $\mathrm{SiO}_{2}$. TIPS-pentacene films suffer from cracking above $320 \mathrm{~K}$ temperature. Hence, all transport measurements with TIPS-pentacene FETs were performed up to $300 \mathrm{~K}$. The thicknesses of the dielectrics were not identical. $\mathrm{Al}_{2} \mathrm{O}_{3}$ was only $30 \mathrm{~nm}$ as it was grown using atomic layer deposition. As a result, the operating voltage of the $\mathrm{Al}_{2} \mathrm{O}_{3}$-based FET was the lowest. Figure 5.5 shows the temperature-dependent transfer curves from TIPS-pentacene FETs using CYTOP, $\mathrm{SiO}_{2}$, and $\mathrm{Al}_{2} \mathrm{O}_{3}$ dielectrics. The carrier mobilities of $\mathrm{SiO}_{2}, \mathrm{Al}_{2} \mathrm{O}_{3}, \mathrm{PVP}$ and CYTOP FETs are extracted at all temperatures and shown in figure 5.5 (d). All of them show activated transport.

$N_{\text {trap }}^{\max }$ may be obtained from the SS of the FET characteristics (Eq. 4.2). By plotting

$N_{\text {trap }}^{\max }$ as a function of temperature for the low $\kappa(\mathrm{CYTOP})$ and high $\kappa\left(\mathrm{Al}_{2} \mathrm{O}_{3}\right)$ dielectric 
(figure 5.5 (e)), we clearly see that the trapped charge density for $\mathrm{Al}_{2} \mathrm{O}_{3}$ is an order of magnitude lower than CYTOP; the lower mobility in $\mathrm{Al}_{2} \mathrm{O}_{3}$-based TIPS-pentacene FETs is, therefore, a manifestation of the high dielectric constant of the gate dielectric.
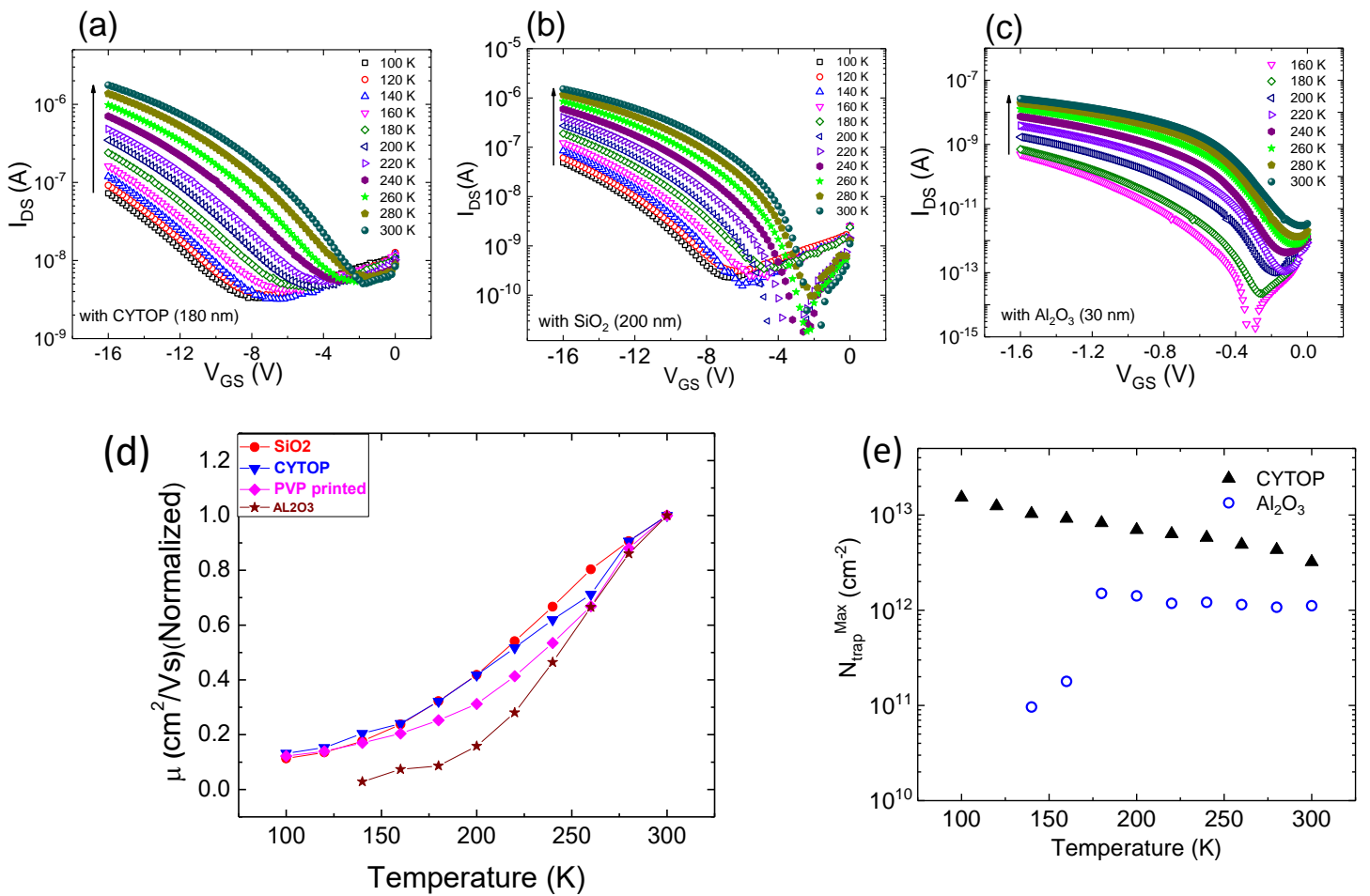

Figure 5.5 (a)-(c) Transconductance curves as a function of temperature from TIPS-pentacene FETs with CYTOP, $\mathrm{SiO}_{2}$ and $\mathrm{Al}_{2} \mathrm{O}_{3}$ dielectrics. (d) $\mu_{\mathrm{FET}}$ mobilities as a function of temperature for $\mathrm{SiO}_{2}, \mathrm{Al}_{2} \mathrm{O}_{3}, \mathrm{PVP}$ and CYTOP dielectrics. (e) The maximum trap density obtained from subthreshold swing values in CYTOP and $\mathrm{Al}_{2} \mathrm{O}_{3}$ based TIPS-pentacene FETs.

\subsubsection{Temperature-dependent FET characteristics with PVDF-TrFE}

TIPS-pentacene FETs using PVDF-TrFE were fabricated both with top contact and bottom contact geometries. Figure 5.6 (a) shows the transconductance curves from a top contact (bottom-gate PVDF-TrFE) TIPS-pentacene FET at various temperatures. The inset shows the high temperature data beyond $160 \mathrm{~K}$. Beyond $240 \mathrm{~K}$, the slopes of the transfer 
curves are clearly seen to decrease. This trend is fully reversible. Whether the measurements are performed from low to high or high to low temperatures, the slopes of the transfer curves are seen to decrease beyond $240 \mathrm{~K}$. These temperature dependent transfer measurements were performed at two different $\mathrm{V}_{\mathrm{DS}}$ values of $-10 \mathrm{~V}$ and $-20 \mathrm{~V}$; correspondingly $\mathrm{V}_{\mathrm{GS}}$ was also swept to $-10 \mathrm{~V}$ or $-20 \mathrm{~V}$. The extracted carrier mobilities are shown in figure 5.6 (b) for the two different $V_{D S}$ values. For both sweeps, the mobilities decrease beyond $240 \mathrm{~K}$. At lower temperatures the mobility is clearly a function of the electric field, as expected from Eq. (5.4). However, beyond $240 \mathrm{~K}$, both low and high field sweeps yield almost the same values of $\mu$ highlighted by the dashed oval, which is a signature of band-like transport. Several PVDF-TrFE single-layer FETs were fabricated, and they all show the same trend i.e., increase in mobility until approximately $220 \mathrm{~K}$ and then a decrease in $\mu$ values.
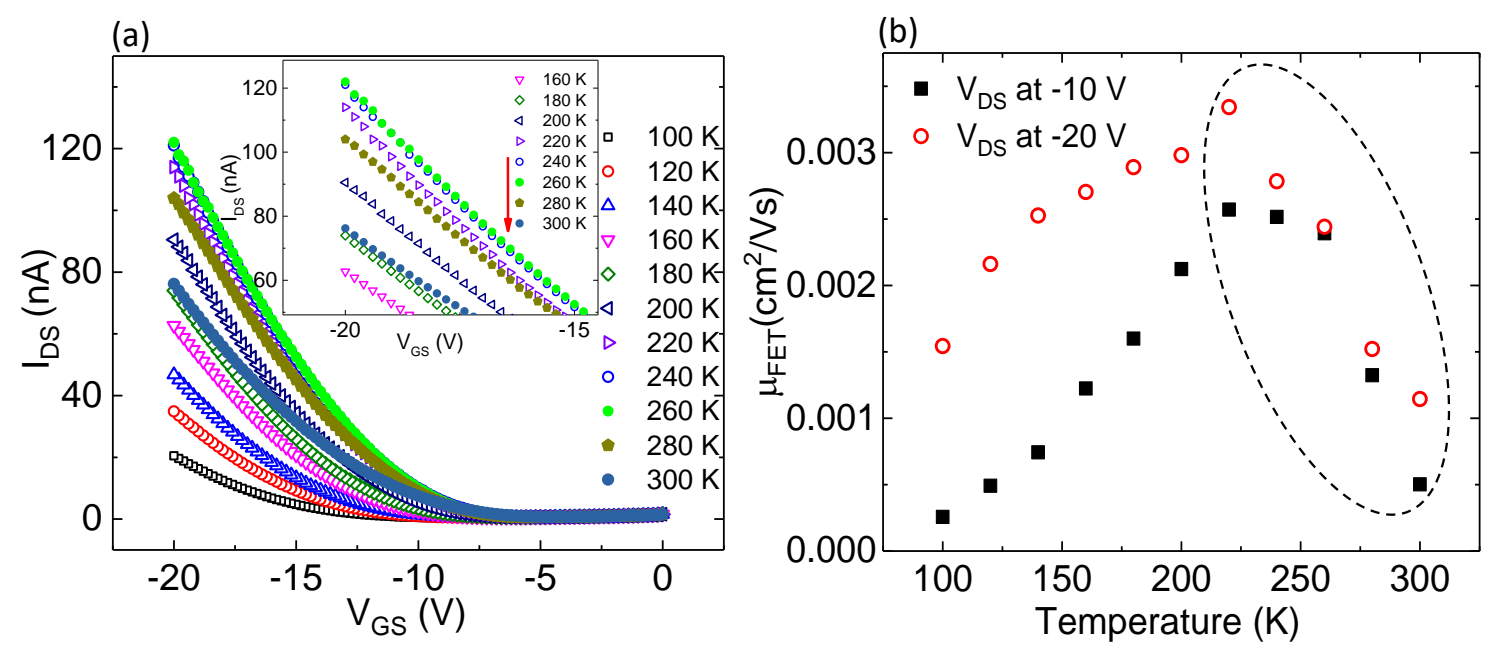

Figure 5.6 (a) Transconductance curves as a function of temperature from TIPS-pentacene FET with PVDFTrFE as the dielectric layer. The inset magnifies the transfer curves upwards of $160 \mathrm{~K}$. (b) The carrier mobilities for PVDF-TrFE based TIPS-pentacene FET extracted from transconductance curves swept at $V_{D S}$ $=-10 \mathrm{~V}$ and $-20 \mathrm{~V}$. The dashed oval highlights the decreasing trend in $\mu$ beyond $240 \mathrm{~K}$. 
Another batch of FETs was fabricated with PVDF-TrFE as the bottom-gate and CYTOP as the top-gate (figure 5.7 (a) inset). The advantage of this geometry in is that it is the same TIPS-pentacene film which is the active layer either with CYTOP or PVDF-TrFE as the dielectric layer. Figure 5.7 (a) plots $\mu$ as a function of temperature both for CYTOP and for PVDF-TrFE. A clear activated transport is seen in CYTOP whereas a decrease in $\mu$ with increasing temperatures beyond $240 \mathrm{~K}$ is observed when the PVDF-TrFE dielectric is gated.

The channel lengths of our FETs are 50 -100 $\mu \mathrm{m}$. The transistor performance is usually contact limited for channel lengths $\leq 10 \mu \mathrm{m}$. For the PVDF-TrFE devices, the contact resistance was roughly two orders of magnitude smaller than the channel lengths. The leakage (gate) current was three orders of magnitude smaller than the drain current for PVDF-TrFE and other dielectrics as well (figure 5.7 (b)), and did not change much with temperature. This ensures that the extracted values of $\mu$ are not affected by the gate current.
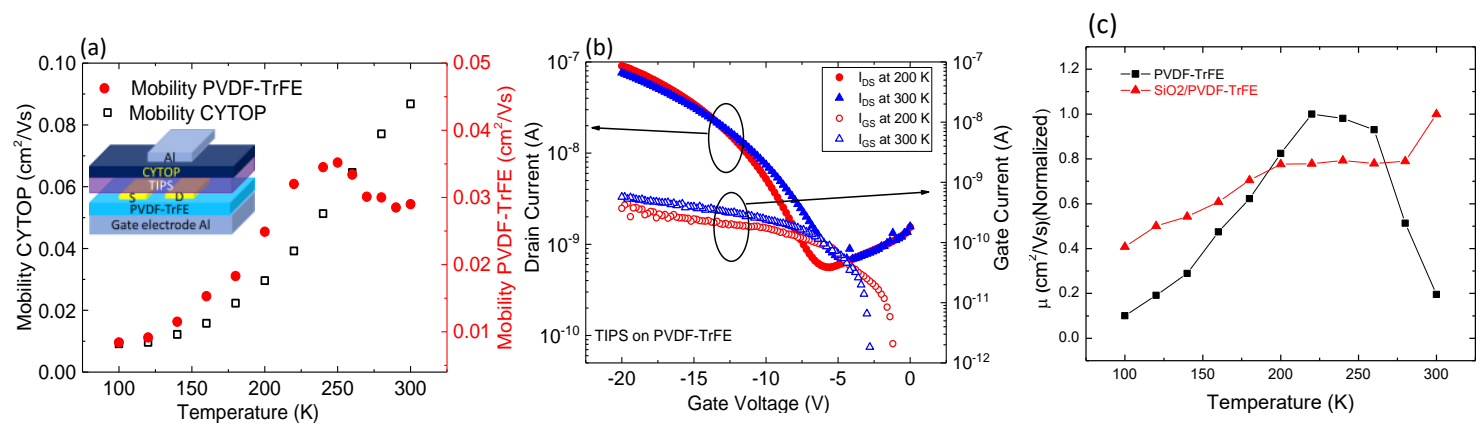

Figure 5.7 (a) The carrier mobility of TIPS-pentacene FET with CYTOP as the dielectric (left axis) and PVDF-TrFE dielectric (right axis) for the dual geometry device shown in the inset. (b) Transfer sweeps at $200 \mathrm{~K}$ and $300 \mathrm{~K}$ for TIPS-pentacene FET (with PVDF-TrFE) showing both drain and the leakage current. (c) The carrier mobility of TIPS-pentacene FET with PVDF-TrFE and SiO2/PVDF-TrFE bilayer as the dielectric. 
We also use bilayer dielectrics $\mathrm{SiO}_{2} / \mathrm{PVDF}-\mathrm{TrFE}$ where the interface property between TIPS-pentacene and the PVDF-TrFE layer is similar to the single layer PVDFTrFE FET, except that, in the bilayer device, the PVDF-TrFE layer does not act as a ferroelectric layer. For a better visualization of FET carrier mobilities as a function of temperature, we plot the normalized values in figure 5.7 (c). A clear distinction is seen between bilayer $\left(\mathrm{SiO}_{2} / \mathrm{PVDF}-\mathrm{TrFE}\right)$ and single-layer PVDF-TrFE FETs. It is important to realize that, in the bilayer device, since the $\mathrm{SiO}_{2}$ layer is gated, PVDF-TrFE is not an active ferroelectric layer; the combined $\mathrm{K}$ is much smaller than for either of the dielectrics. Although in both FETs the interface between TIPS-pentacene and PVDF-TrFE is identical, the bilayer device does not show $\mathrm{d} \mu / \mathrm{dT}<0$, suggesting that the ferroelectric nature of PVDF-TrFE is responsible for the negative coefficient of mobility beyond $220 \mathrm{~K}$.

The nature of transport in TIPS-pentacene FETs is quite different between nonferroelectric and ferroelectric dielectrics. Using the concept of the mobility edge, introduced in section 5.1.1, and the nature of discrete shallow traps in TIPS-pentacene, one can rationalize the observed band-like transport in TIPS-pentacene FETs with PVDFTrFE. We note that bulk transport in TIPS-pentacene shows $\mathrm{d} \mu / \mathrm{dT}<0$. This trend is seen in other high purity crystals of the acene family such as anthracene and tetracene; discrete trap SCLC typically displays diminishing mobility with increasing temperature. ${ }^{69}$ In TIPSpentacene FETs, the polarization field of PVDF-TrFE beyond $200 \mathrm{~K}$ may partially reduce the trap depth due to screening, resulting in a de-trapping mechanism. The density of discrete traps may then approach the mobility edge; the low energetic disorder in TIPSpentacene can be overcome, leading to a diffusive transport. 
We further estimate the energetic disorder $\left(\sigma^{\prime}\right)$ by fitting the temperature-dependent $\mu$ data of unpoled PVDF-TrFE using Eq. 5.3 as shown in figure 5.8. In the temperature range where a true hopping transport is observed (from $100-220 \mathrm{~K}$ ), the sweeps at two different values of $\mathrm{V}_{\mathrm{DS}}$ are well-below $2 k_{B} \mathrm{~T}$, and thus one may justify extracting the carrier mobilities for TIPS-pentacene FETs from the saturation region of the transfer curves. We also fit the high temperature region where a band-like transport is observed with $\mu \propto \mathrm{T}^{-\mathrm{n}}$; $\mathrm{n}$ is found to be 2.7 , similar to values observed in ordered organic semiconductors.

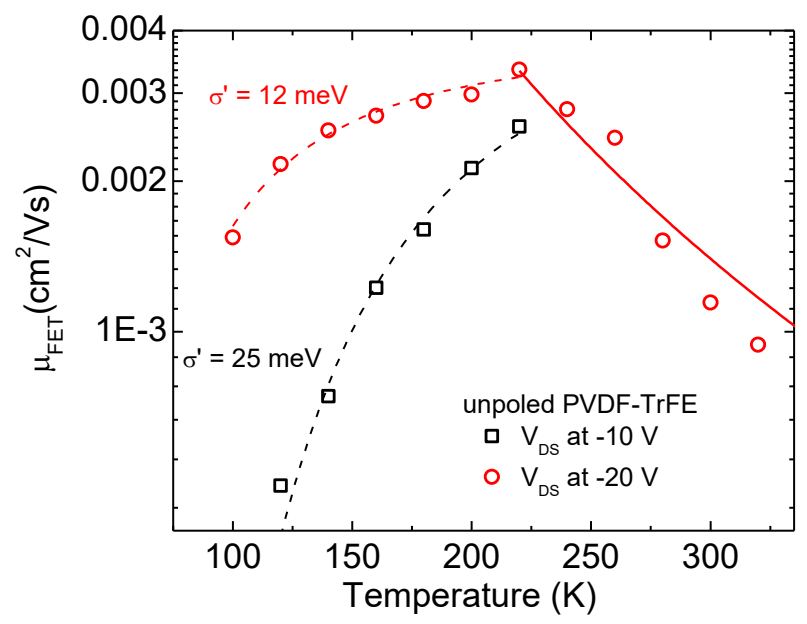

Figure 5.8 Fits to the experimental TIPS-pentance $\mu$ data using unpoled PVDF-TrFE as the dielectric. The temperature range between $100-220 \mathrm{~K}$ is fit with Eq. 5.4. The high temperature range for the sweep at $\mathrm{V}_{\mathrm{DS}}$ at $-20 \mathrm{~V}$ has been fit with $\mu \propto \mathrm{T}^{-\mathrm{n}}$.

Poling of the ferroelectric dielectric layer with an external field is seen to benefit transistor performance. ${ }^{83}$ We next look at the temperature dependence of $\mu$ with vertically poled PVDF-TrFE. PVDF-TrFE was vertically poled using a temporary top electrode as discussed in section 6.1.2. Figure 5.9 (a) shows $\mu$ as a function of temperature from both unpoled and vertically poled PVDF-TrFE. In order to understand the differences between unpoled PVDF-TrFE and its vertically poled counterpart, the trapped charge density was 
estimated from the subthreshold swing of the FET characteristics. Figure 5.9 (b) plots $N_{\text {trap }}^{\max }$ for unpoled (swept at two different values of $V_{D S}$ ) and poled PVDF-TrFE FETs. Above $200 \mathrm{~K}$, the trap density is seen to be an order of magnitude higher for the poled dielectric film. The nature of transport with poled PVDF-TrFE is similar to other nonferroelectric dielectrics. These observations suggest that the energy fluctuations due to the randomly oriented dipoles that are present locally in the unpoled PVDF-TrFE films, and not the trapped charge density, are responsible for the band-like transport as well as lower values of $\mu$ compared to poled PVDF-TrFE, seen above temperatures of $200 \mathrm{~K}$. We want to point out that the contact resistance of FETs used in this work are minimal as the channel lengths are relatively large $(50-100 \mu \mathrm{m})$. The contact resistance was at least two orders of magnitude smaller than the channel resistance, and the difference between the poled and unpoled FETs were minimal. Inset in figure 5.9 (b) shows the variation of threshold voltage in unpoled and vertically poled PVDF-TrFE FETs.

(a)

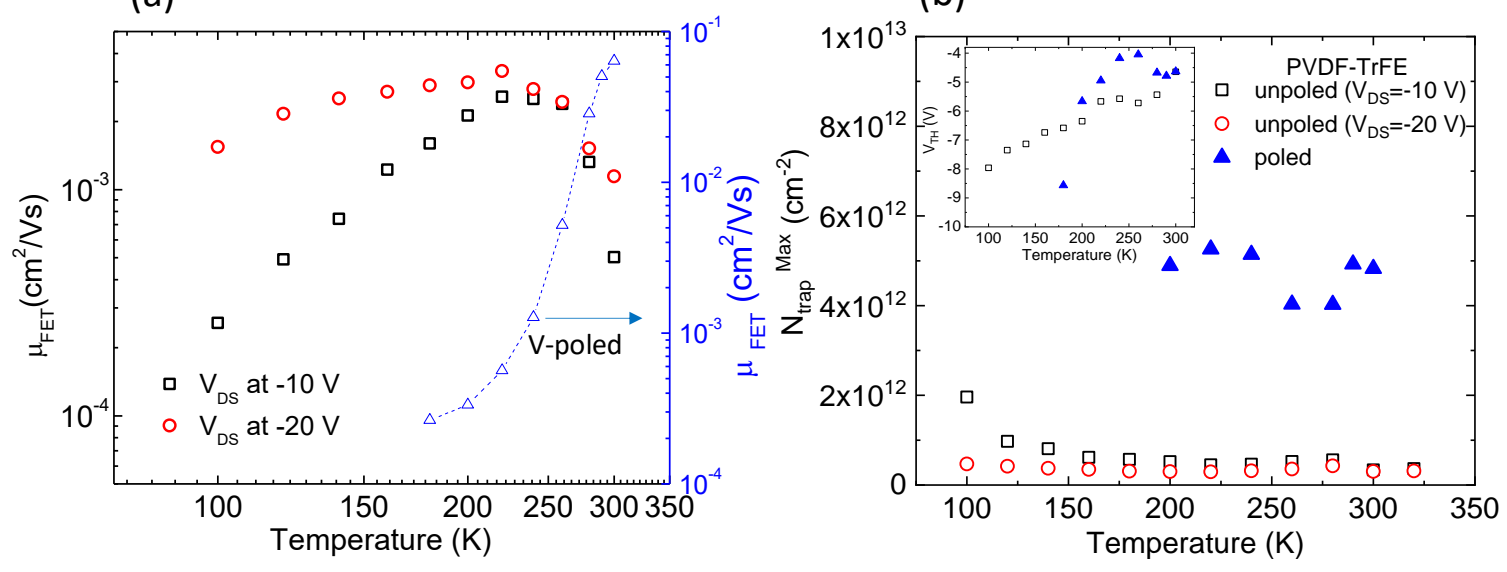

Figure 5.9 (a) TIPS-pentacene FET mobility as a function of temperature at two different $V_{D S}$ values for unpoled PVDF-TrFE. The blue triangles are the FET mobility from a TIPS-pentacene FET where the PVDF- 
TrFE dielectric layer was vertically poled. (b)The trapped charge density as a function of temperature for TIPS-pentacene FETs using unpoled and vertically poled PVDF-TrFE.

In conclusion, polarization fluctuation inherent to PVDF-TrFE influences transport in other organic FETs as well. Drop-casted TIPS-pentacene films exhibit single-trap-level SCLC, similar to high-purity crystalline organic molecules. A bulk-transport measurement from TIPS-pentacene clearly shows a negative coefficient of mobility beyond $180 \mathrm{~K}$. A similar behavior is seen in FET transport when a ferroelectric dielectric, PVDF-TrFE, is used. The presence of a polarization field of the ferroelectric dielectric results in a transport mechanism, exemplifying a band-like transport above a certain temperature. By fitting the high temperature regime of unpoled PVDF-TrFE devices to $\mu=\mathrm{T}^{-\mathrm{n}}$, $\mathrm{n}$ was found to be 2.7, similar to values obtained for ordered organic semiconductors. All other non-ferroelectric dielectrics and poled PVDF-TrFE (where the dipoles are prealigned) show an activated transport similar to $\mathrm{Al}_{2} \mathrm{O}_{3}$, suggesting that the reduced mobility with unpoled PVDF-TrFE is not just due to the long-range polaron coupling but rather due to its inherent polarization fluctuation in conjunction with the nature of discrete trap-states in TIPS-pentacene. 


\section{Dipole engineering of ferroelectric dielectrics- enhancement in FET performance}

In chapters 4 and 5 we gave several examples to show the influence of PVDF-TrFE in organic FETs. This helps us to understand the charge transport mechanism in such devices. The formation of Fröhlich polarons suppresses the FET mobility, reducing the performance of such FETs. PVDF-TrFE, in spite of its applications in vast range of work including as a gate dielectric in organic $\mathrm{FETs}^{48,60,84,85}$ and sensing applications ${ }^{86,87}$, pose some concerns. These concerns are related to its inherent surface roughness issues as well as higher conductivity compared to other polymer non-ferroelectric dielectrics; the DC conductivity of PVDF (of approximately $10^{-12} \mathrm{~S} / \mathrm{m}$ ) is a few orders higher than other polymer dielectrics such as poly-methylmethacrylate (PMMA) ${ }^{88}$ Additionally, the dipolar disorder at the semiconductor-PVDF-TrFE interface in FET architectures is higher compared to other polymer ferroelectrics. As a result PVDF-TrFE as a dielectric layer in organic FETs show low carrier mobilities in comparison to non-ferroelectric polymer dielectrics. ${ }^{85}$ To use PVDF-TrFE as a gate dielectric, we thus need to enhance the FET performance. In this chapter we will discuss the techniques developed by us to enhance the performance of organic FETs performance based on PVDF-TrFE. We use the method of dipole engineering of the PVDF-TrFE film. In particular we have come up with new methods of electrical poling the dielectric layer to enhance FET performance as well as reduce gate leakage issues. This chapter includes two parts: (a) polarization rotation in the dielectric PVDF-TrFE and (b) textured poling of dielectric PVDF-TrFE. 


\subsection{Polarization rotation in the dielectric PVDF-TrFE}

\subsubsection{Introduction}

A juxtaposition of ferroelectric polymers and ferroelectric oxides shows MOSFETs have greatly benefited from the intrinsic spontaneous electric polarization of ferroelectric oxides. Polarization modulation in ferroelectric oxides has enabled fast switching and lowpower operation in MOSFETs. ${ }^{89,90}$ The spontaneous electric polarization of ferroelectric oxides not only controls the channel conductance but may be further reoriented by the application of an external electric field. ${ }^{91,92}$ Although the polarization distribution effect of the ferroelectric oxide on the channel current-gate voltage has been considered in MOSFETs. ${ }^{93}, 94$ There are no such parallel studies on polymer ferroelectric FETs, especially where the effect of rotating the polarization direction by $90^{\circ}$ on FET performance has been examined.

Theoretical work shows the effect of polarization rotation on the performance of MOSFETs using $\mathrm{BaTiO}_{3}$ as the gate insulator. ${ }^{35}$ Polarization rotation changes the surface potential of the silicon substrate, resulting in a subthreshold swing (SS) lower than 60 $\mathrm{mV} /$ decade by a judicious choice of the thickness of the gate dielectric layer. It is seen that polarization rotation (from out-of-plane to in-plane) offers significant advantages over polarization inversion in the $\mathrm{BaTiO}_{3}$ layer. Spontaneous polarization during the reorientation increases the screening charge accumulation in the MOSFET channel, resulting in low SS. Although the SS of FETs based on polycrystalline organic films have now paralleled those of MOSFETs with values close to $90 \mathrm{mV} / \mathrm{decade},{ }^{36,37}$ they usually involve a self-assembled monolayer on high-quality oxide dielectrics such as $\mathrm{AlO}_{\mathrm{x}}$. In 
single crystal rubrene FETs, the disorder is significantly reduced with interface trap densities similar to that in silicon transistors, and SS values of $65 \mathrm{mV} / \mathrm{dec}$ ade have been achieved. ${ }^{38}$ A question that arises is whether polarization reorientation in a polymer ferroelectric dielectric is an effective mechanism for improving SS and other transistor parameters in organic FETs. Specifically, we are interested in changes in SS and other FET parameters by rotating the polarization direction of the dielectric by $90^{\circ}$. Polarization rotation is different from polarization inversion, which in principle may be achieved by sweeping the gate from high negative to positive voltages in ferroelectric organic FETs. Such inversion effects have benefits in changing the on/off ratio for memory application.

By using two different organic molecules: DNTT and pentacene, and solution processable semiconductor TIPS-pentacene along with unpoled and poled PVDF-TrFE as the gate dielectric, in top-contact bottom-gate FET architectures, carrier transport is investigated. Prior to depositing the organic semiconductor, the ferroelectric film is either vertically or laterally poled by applying an external electric field while heating the polymer just above its ferroelectric-paraelectric transition temperature at $135^{\circ} \mathrm{C}$, which is known to increase the degree of crystallinity. ${ }^{95}$ The presence of additional lateral electrodes with vertically poled FETs facilitates the application of a lateral electric field, which helps to monitor transistor properties as a function of reorientation of the polarization direction by $90^{\circ}$ in the dielectric medium. Since we investigate $p$-type transport, the poling direction is appropriately chosen so that the operating field does not work against the poling field. The FETs with vertically poled PVDF-TrFE film perform the best with the lowest value of SS. As a lateral electric field on the top surface is applied to the vertically poled FETs, the transistor characteristics are seen to degrade. The phenomenon is reversible; by applying a 
lateral field in the opposite direction, the transistor properties recover. The FETs operate at lower voltages compared to the coercive voltage of the PVDF-TrFE layer, ensuring that the changes observed in the FET properties arise from external poling conditions. The poling field controls the microstructure of PVDF-TrFE, which is inferred from $\mathrm{x}$-ray diffraction (XRD) and electron microscopy images. Density-functional-theory (DFT) studies from a monomer unit embedded in a dielectric medium under the application of both vertical and horizontal electric fields shed light on the changes in the net dipole moment and structure (deviation from the all-trans phase) of the molecule. This study highlights new design principles for organic FETs in lowering SS and enhancing transport properties by orienting the polarization direction by $90^{\circ}$ in the ferroelectric dielectric layer.

\subsubsection{Poling of PVDF-TrFE films}

PVDF-TrFE is dissolved in the solvent $\mathrm{N}, \mathrm{N}$-dimethylformamide $(50 \mathrm{mg} / \mathrm{ml})$ (DMF). This dielectric solution is then spin casted on top of the Al-coated glass at a spin speed of $1600 \mathrm{rpm}$ for $60 \mathrm{sec}$ and heated at $70^{\circ} \mathrm{C}$ for 10 minutes to remove the solvent residue. The PVDF-TrFE films are then annealed at $135^{\circ} \mathrm{C}$ for half an hour for further enhancement of the $\beta$ phase of the copolymer. A temporary electrode is prepared by depositing $300 \mathrm{~nm}$ of $\mathrm{Al}$ on a glass slide. Electrical contacts are made, and the slide is placed (with the Al strip facing down) on top of the PVDF-TrFE film, as shown in figure 6.1(a). A 50-g weight is added on top to ensure a uniform contact of the top electrode with the ferroelectric surface. PVDF-TrFE films are vertically poled by applying an electric field of $\sim 100 \mathrm{MV} / \mathrm{m}$ during the crystallization process. The field is on while the film is allowed to slow cool for half an hour. Two aluminum strips, $2.0 \mathrm{~mm}$ apart, are deposited 
on a few films by the shadow mask method as lateral poling electrodes. We note that the lateral electrodes are deposited on top of the PVDF-TrFE film whereas the gate electrode is placed under the dielectric layer. Additionally, there is no overlap between the top lateral electrodes and the gate electrode (as shown in Fig. 6.1(b)). During lateral poling, the voltage is only applied to the lateral electrodes. Hence, the electric field should be uniform during lateral poling. An electric field of about $0.1 \mathrm{MV} / \mathrm{m}$ is applied laterally to these films for 15 minutes at $135^{\circ} \mathrm{C}$. Lateral poling electrodes are also deposited on vertically poled films to access lateral poling during the device characterization. For MIM capacitors, unpoled, vertically poled (V-poled), and laterally poled (L-poled) PVDF-TrFE films on Al-coated glass are followed by $60 \mathrm{~nm}$ aluminum or gold through a shadow mask to complete the MIM structure.

(a)

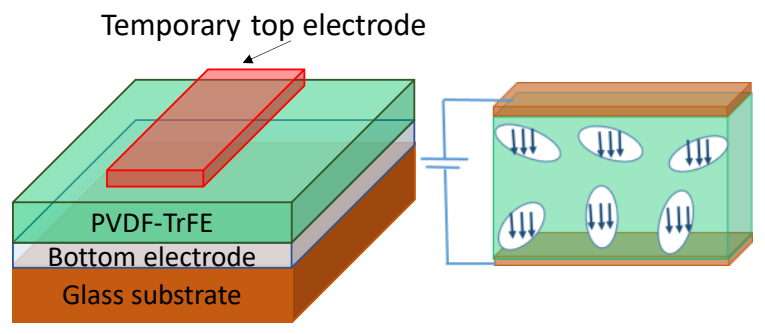

(b)

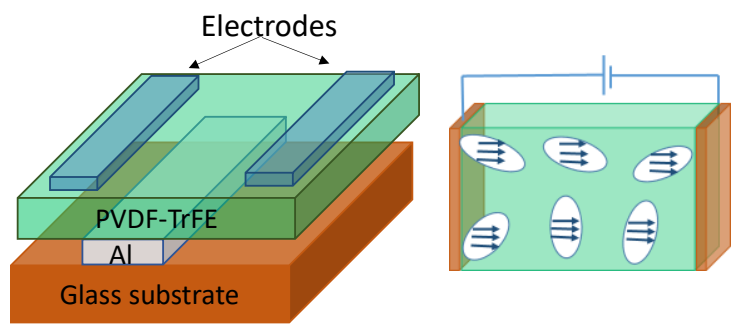

Figure 6.1 Poling of PVDF-TrFE films. (a) Schematic of vertical and (b) Lateral poling of PVDF-TrFE, respectively. Arrows denote the polarization direction in the crystalline phases of the ferroelectric slab after poling. 


\subsubsection{Small-molecule FETs with poled and unpoled PVDF-TrFE}

DNTT FETs are prepared on three different substrates with unpoled, V-poled, and L-poled PVDF-TrFE films. The film thickness of the dielectric and the semiconducting layer are identical for all three substrates.

(a)

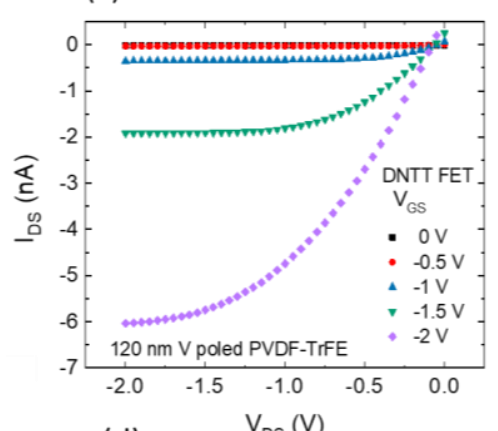

(d)

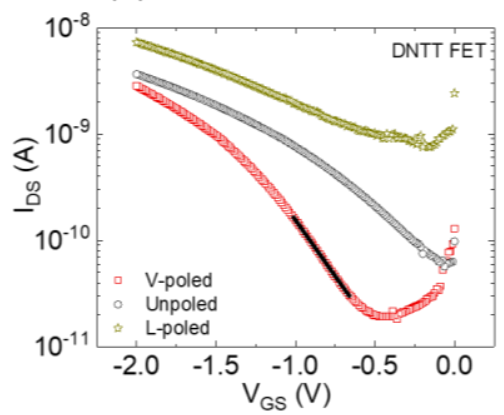

(b)

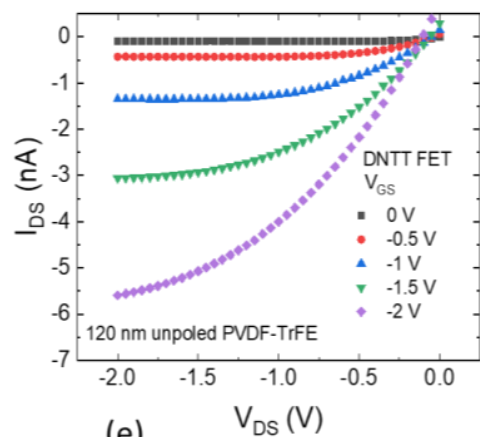

(e) $\quad V_{D S}(V)$

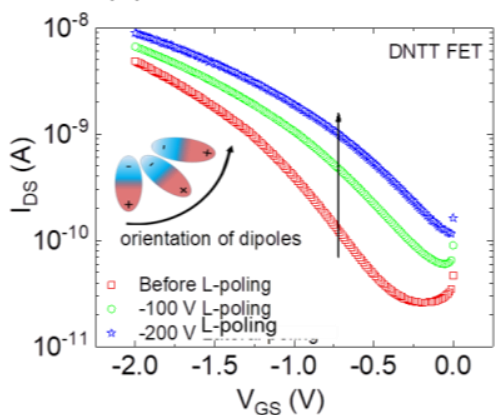

(c)
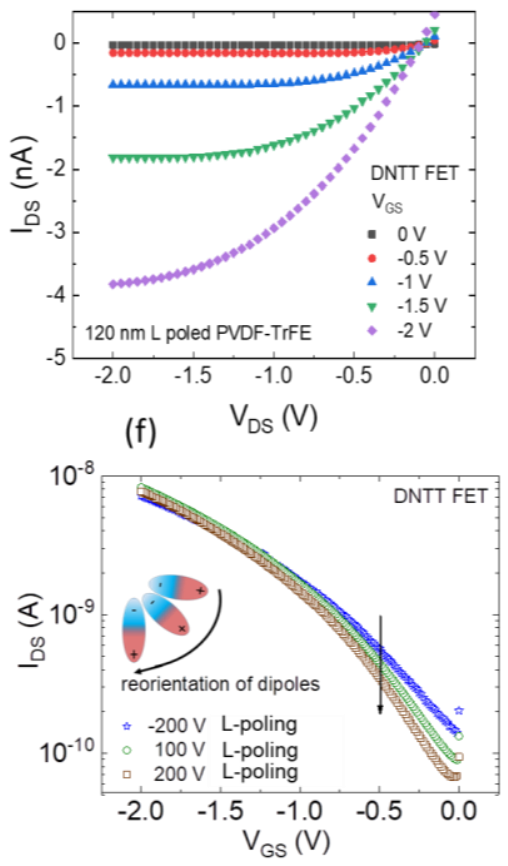

Figure 6.2 Output and Transfer characteristics of DNTT-based FETs. (a-c) Output characteristics of DNTT FET with unpoled, V-poled, and L-poled PVDF-TrFE (d) Transfer characteristics of DNTT FETs with Vpoled, unpoled, and L-poled PVDF-TrFE. The black line depicts a linear fit to the subthreshold region. (e) Transfer characteristics of a vertically poled FET by applying various magnitudes of a lateral voltage. Green and blue curves depict devices where the lateral field is applied such that the polarization direction of the dielectric is oriented parallel to the external source-drain electric field. (f) Transfer curves of the same FET device shown in (e) after reversing the polarity of the lateral field, such that the polarization direction is oriented antiparallel to the source-drain electric field. 
Figure 6.2 (a-c) show the output characteristics of DNTT FET with unpoled, Vpoled, and L-poled PVDF-TrFE. The device size was identical for all of them $(\mathrm{W}=1 \mathrm{~mm}$ and $\mathrm{L}=100 \mu \mathrm{m})$. Corresponding transfer characteristics of these DNTT FETs with $\mathrm{V}$ poled, unpoled, and L-poled PVDF-TrFE are shown in figure 6.2 (d). These are all swept in the saturation region where the drain-source voltage $\left(\mathrm{V}_{\mathrm{DS}}\right)$ is maintained at $-2 \mathrm{~V}$, and the gate voltage $\left(\mathrm{V}_{\mathrm{GS}}\right)$ is varied. Device parameters such as carrier mobility, on-off current ratio, and threshold $\left(\mathrm{V}_{\mathrm{Th}}\right)$ are estimated using the standard saturation regime currentvoltage characteristics. Channel length varied between 50 and $125 \mu \mathrm{m}$ for the FETs. More than the changes in $\mu$, the subthreshold swing and other FET parameters, as discussed below, vary with the poling condition; hence, we quote only the saturation mobilities. Work by Choi et al. defines the reliability factor $r$ for extracting carrier mobilities both in the saturation and linear regions of the transfer characteristics. The $r$ factor is defined based on the ideal Shockley FET equations with $\mathrm{V}_{\mathrm{Th}}=0$ and can be expressed as ${ }^{96}$,

$$
r=\left\{\frac{\sqrt{\left|I_{D S}^{M a x}\right|}-\sqrt{I_{D S}^{o}}}{\left|V_{G S}^{M a x}\right|}\right\}^{2} /\left(\frac{\partial \sqrt{I_{D S}}}{\partial V_{G S}}\right)^{2}
$$

Based on this definition, the $r$ factor for $\mu_{\text {sat }}(\mathrm{V}$-poled FET) is found to be $\sim 47 \%$. We point out that for ferroelectric dielectrics the value of $\mathrm{V}_{\mathrm{Th}}$ can greatly vary, from being positive to negative, as discussed later. Hence, one cannot expect an ideal $r$ factor of $\sim 100 \%$.

The SS varies for the unpoled, V-poled, and L-poled PVDF-TrFE FETs. A representative linear fit (black line) for the V-poled device is shown in figure 6.2(d). We 
note that this fit just represents the subthreshold region used for obtaining SS; the carrier mobilities are obtained as outlined above. It may be more appropriate to compare the normalized equivalent of SS by taking into account the capacitance of the insulator ( $\mathrm{SS} i=$ $\mathrm{SS} \times C O$ ), as defined for single-crystal FETs. ${ }^{97}$ The FET parameters including $\mathrm{SS}_{\mathrm{i}}$ are tabulated in Table 6-I. Several devices are tested from where the error bar are estimated.

Table 6-I The FET parameters extracted from unpoled V-poled, and L-poled PVDF-TrFE from DNTT and pentacene FETs.

\begin{tabular}{ccccc}
\hline Semiconductor & $\begin{array}{c}\text { Dielectric } \\
\text { poling condition }\end{array}$ & $\begin{array}{c}\text { Subthreshold } \\
\text { swing (SS) } \\
(\mathrm{mV} / \mathrm{dec})\end{array}$ & $\begin{array}{c}\text { Normalized SS } \\
(\mathrm{SSi}) \\
\left(\mathrm{nFV} / \mathrm{dec} \mathrm{cm}^{2}\right)\end{array}$ & $\begin{array}{c}\text { Mobility } \\
\left(\times 10^{-3} \mathrm{~cm}^{2} / \mathrm{Vs}\right)\end{array}$ \\
\hline \multirow{3}{*}{ DNTT } & V-poled & $403 \pm 5$ & 37 & $11.3 \pm 0.2$ \\
& Unpoled & $1000 \pm 8$ & 62 & $6.0 \pm 0.1$ \\
& L-poled & $1700 \pm 11$ & 105 & $5.0 \pm 0.1$ \\
\cline { 2 - 5 } Pentacene & V-poled & $600 \pm 4$ & 55 & $2.9 \pm 0.1$ \\
& Unpoled & $1100 \pm 7$ & 68 & $1.6 \pm 0.2$ \\
& L-poled & $1140 \pm 9$ & 71 & $1.5 \pm 0.1$ \\
\hline
\end{tabular}

The FET with the V-poled PVDF-TrFE films shows the lowest value of SS and the highest value of $\mu$. The L-poled FET performs worse than the unpoled device, which is an indication that there is some orientation of the dipoles in the vertical direction in unpoled PVDF-TrFE films.

Pentacene FETs with V-poled, unpoled, and L-poled PVDF-TrFE are also fabricated. The transfer curves (in the saturation region) are shown in figure 6.3 (a), and their SS and $\mu$ values are tabulated in Table 6-I. The device performance trends are identical to DNTT FETs; the V-poled pentacene FET performs the best with the lowest value of SS and the highest value of $\mu$. TIPS-pentacene FETs are also fabricated with V-poled and 
unpoled PVDF-TrFE; one of the lowest values of SS ( $<200 \mathrm{mV} / \mathrm{dec})$ is observed for Vpoled PVDF-TrFE TIPS-pentacene FET (figure 6.3 (b)).

(a)

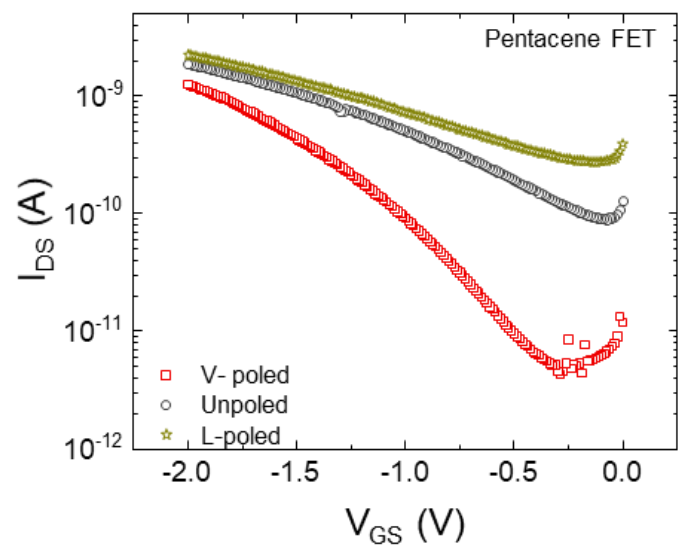

(b)

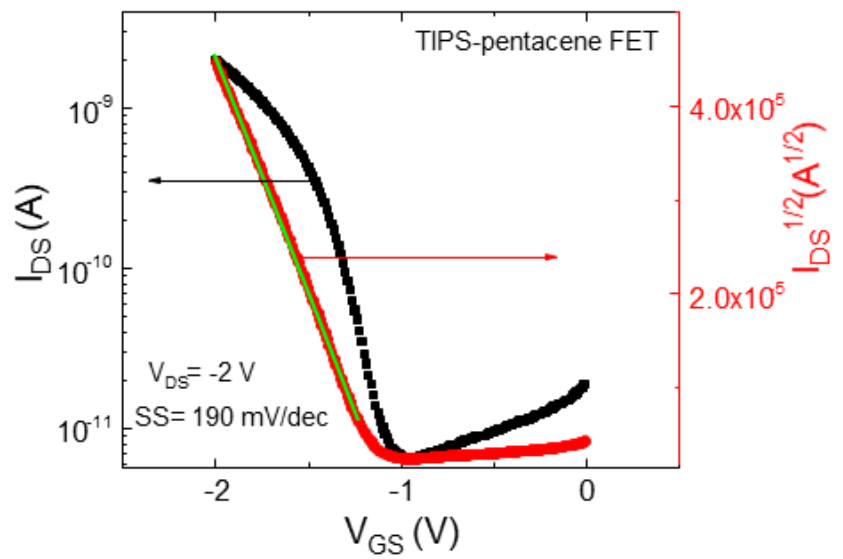

Figure 6.3 (a) Transfer characteristics of pentacene FETs with V-poled, unpoled, and L-poled PVDF-TrFE

(b) Transfer curve from V-poled TIPS-pentacene based FET. All curves were swept in the saturation region regime where the drain-source voltage $\left(\mathrm{V}_{\mathrm{DS}}\right)$ was maintained at $-2 \mathrm{~V}$, and the gate voltage $\left(\mathrm{V}_{\mathrm{GS}}\right)$ was varied from 0 to $-2 \mathrm{~V}$.

The general trend is that V-poled PVDF-TrFE FETs show the lowest off current with improved SS. An improvement in carrier mobility is also observed for all V-poled devices. Since the direction of polarization (P) in V-poled PVDF-TrFE film is in the same direction as the gate electric field, it enhances the screening charge accumulation in the channel region improving transport, resulting in a lower value of SS. Concomitantly, the accumulation capacitance in a MIS diode is seen to increase for the V-poled film. Capacitance-voltage $(C-V)$ sweeps from DNTT and pentacene MIS structures (with unpoled and V-poled PVDF-TrFE films) are shown in figure 6.4. In both cases, the capacitance in the accumulation region is higher when the PVDF-TrFE film is V-poled 
compared to the unpoled case. The change in capacitance is also seen in MIM structures with different poling conditions of the insulator, as discussed later.
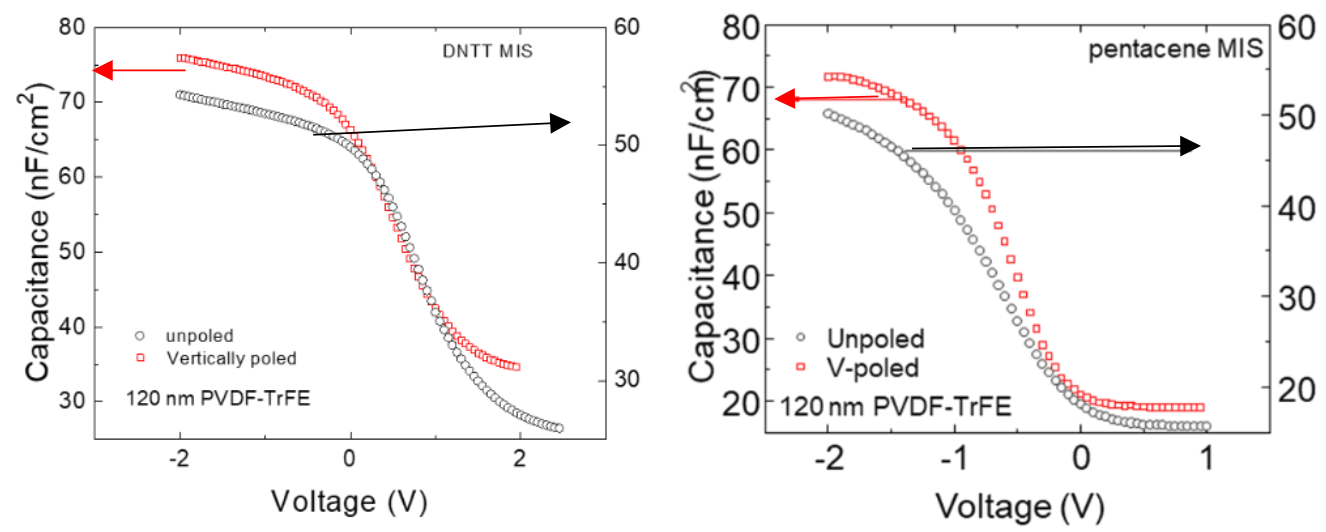

Figure 6.4 Typical capacitance versus voltage characteristics of (a) DNTT MIS (b) pentacene MIS devices.

A question that arises is whether the FET properties are tunable by changing the polarization direction of the dielectric medium. The V-poled FETs are incorporated with lateral electrodes (at a separation of $\sim 2 \mathrm{~mm}$ ). The lateral electric field is applied for 15 minutes and its magnitude is in the range of $10^{4}-10^{5} \mathrm{~V} / \mathrm{m}$. Figure $6.2(\mathrm{c})$ shows the transfer curves measured after the application of a lateral electric field (with varying magnitude) to the V-poled sample. The direction of $\mathbf{P}$ (with the lateral field) is in the same direction of the source-drain electric field $\left(\mathbf{E}_{D S}\right)$. As the vertical polarization is reoriented in the horizontal direction, it results in degradation of the FET properties, which is seen in the transfer curves. The tabulated values of SS and $\mu$ are shown in Table 6-II. We note that the DNTT V-poled FETs in Table 6.I are a different batch compared to the V-poled DNTT FET shown in Table 6-II. The initial L-poled FET has a larger SS compared to L-poling a 
V-poled FET device. Since the original V-poled film is already crystallized, applying only a lateral field may not have the same effect as applying a lateral field during crystallization.

Table 6-II FET parameters for a vertically poled PVDF-TrFE film using DNTT as the organic semiconductor. Lateral electric field is applied in the direction of $\mathbf{E}_{\mathrm{DS}}$, and then the polarity is reversed.

\begin{tabular}{lccc}
\hline $\begin{array}{c}\text { Dielectric poling } \\
\text { condition }\end{array}$ & $\begin{array}{c}\text { Subthreshold } \\
\text { swing } \\
(\mathrm{mV} / \mathrm{dec})\end{array}$ & $\begin{array}{c}\text { Threshold Voltage } \\
(\mathrm{V})\end{array}$ & Mobility $\left(\times 10^{-3} \mathrm{~cm}^{2} / \mathrm{Vs}\right)$ \\
\hline Vertical & $530 \pm 2$ & -0.78 & 11.00 \\
Lateral $(-50 \mathrm{~V})$ & $580 \pm 3$ & -0.47 & 10.00 \\
Lateral $(-100 \mathrm{~V})$ & $650 \pm 3$ & -0.40 & 9.40 \\
Lateral $(-150 \mathrm{~V})$ & $660 \pm 5$ & -0.27 & 9.20 \\
Lateral $(-200 \mathrm{~V})$ & $700 \pm 7$ & -0.22 & 9.00 \\
& & Polarity reversed & 8.30 \\
Lateral $(100 \mathrm{~V})$ & $650 \pm 4$ & -0.30 & 9.40 \\
Lateral $(200 \mathrm{~V})$ & $580 \pm 3$ & -0.33 & \\
\hline
\end{tabular}

After the FET is L-poled to $\sim 10^{5} \mathrm{~V} / \mathrm{m}(-200 \mathrm{~V})$, the direction of L-poling is reversed [figure 6.2(d)]. The FET properties are seen to recover (also shown in Table 6.II). The L-poling of a V-poled sample, thus, serves as a tuning knob to reorient the polarization by $90^{\circ}$. In order to gain insight into the interface potential for different poling conditions, Grünewald's method ${ }^{98}$, which has been extensively used for estimating the trap density of states, ${ }^{99,100}$ is used. By determining the dependence of $\mathrm{I}_{\mathrm{DS}}$ on the electric field due to $\mathrm{V}_{\mathrm{GS}}$, the gate-dependent dielectric-semiconductor interface potential $V_{0}=V_{0}(U g)$ is determined from,

$$
\left.\exp \left(\frac{e V_{o}}{k T}\right)-\frac{e V_{o}}{k T}-1=\frac{e}{k T} \frac{\epsilon_{i} d}{\epsilon_{s} l \sigma_{0}}\left[U_{g} \sigma\left(U_{g}\right)-\int_{0}^{U_{g}} \sigma\left(\tilde{\mathrm{U}}_{g}\right) d \tilde{\mathrm{U}}_{g}\right)\right]
$$


where e, $\mathrm{k}$, and $\mathrm{T}$ are the elementary charge, the Boltzmann constant, and the absolute temperature, respectively; $\mathrm{Ug}=\left|\mathrm{V}_{\mathrm{GS}}-\mathrm{V}_{\mathrm{FB}}\right|$ and $\mathrm{V}_{\mathrm{FB}}$ is the flat-band voltage; $\sigma(U g)=$ $(L / W)\left(I_{D S} / V_{D S}\right)$ is the field-dependent conductivity, and $\sigma_{0}$ is the conductivity at $U g=0 ; \boldsymbol{\epsilon}_{i}$ and $\boldsymbol{\epsilon}_{s}$ are the dielectric constants of the insulator and the semiconductor. For each gate voltage, Eq. (6.1) is numerically evaluated using the measured field-effect conductivity. $\mathrm{V}_{\mathrm{FB}}$ is assumed to be the onset gate voltage of the device. By rewriting $\mathrm{SS}$ as $\left[\partial \mathrm{V}_{\mathrm{GS}} / \partial \mathrm{V}_{\mathrm{o}}\right]\left[\partial \mathrm{V}_{\mathrm{o}} / \partial \log \left(\mathrm{I}_{\mathrm{DS}}\right)\right]$, the changes in SS may be attributed to an increase in the first term. Further, the total hole density, $\mathrm{p}\left(\mathrm{V}_{\mathrm{o}}\right)$, is dependent on the interface potential $\left[\mathrm{p}\left(\mathrm{V}_{\mathrm{o}}\right)\right.$ $\left.\propto\left(\mathrm{dV}_{\mathrm{o}} / \mathrm{dUg}\right)-1\right]$. Figure 6.5 (a) shows the interfacial potential as a function of Ug. Upon taking the derivative of the curves (and inverting), we see that $\partial \mathrm{Ug} / \partial \mathrm{V}_{\mathrm{o}}$ increases after $\mathrm{L}$ poling (at $-50 \mathrm{~V}$ and $-200 \mathrm{~V}$ ), corroborating the increase in SS seen in the FETs (after Lpoling). As shown in figure 6.5 (b), it is observed that upon L-poling the V-poled film, the $\mathrm{p}\left(\mathrm{V}_{\mathrm{o}}\right)$ decreases. ${ }^{101}$ This decrease suggests more trapping upon L-poling and a reduction in the carrier mobility.
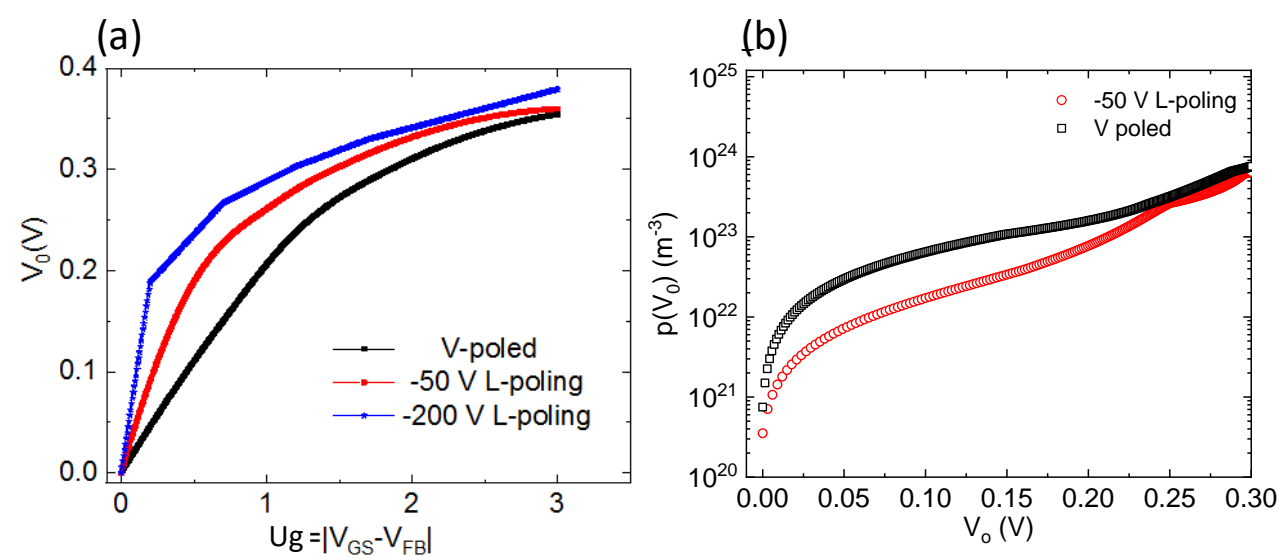

Figure 6.5 Interface potential and total hole density. (a) The interface potential versus $U_{g}$, the gate voltage above the flat band voltage. The black curve is for a V-poled PVDF-TrFE DNTT FET. The red and blue 
curves are after L-poling the dielectric layer by applying a $-50 \mathrm{~V}$ and $-100 \mathrm{~V}$ to the dielectric as explained in the text. (b) The hole density as a function of the interface potential for a V-poled and L-poling (by applying $-50 \mathrm{~V})$ of the V-poled DNTT FET.

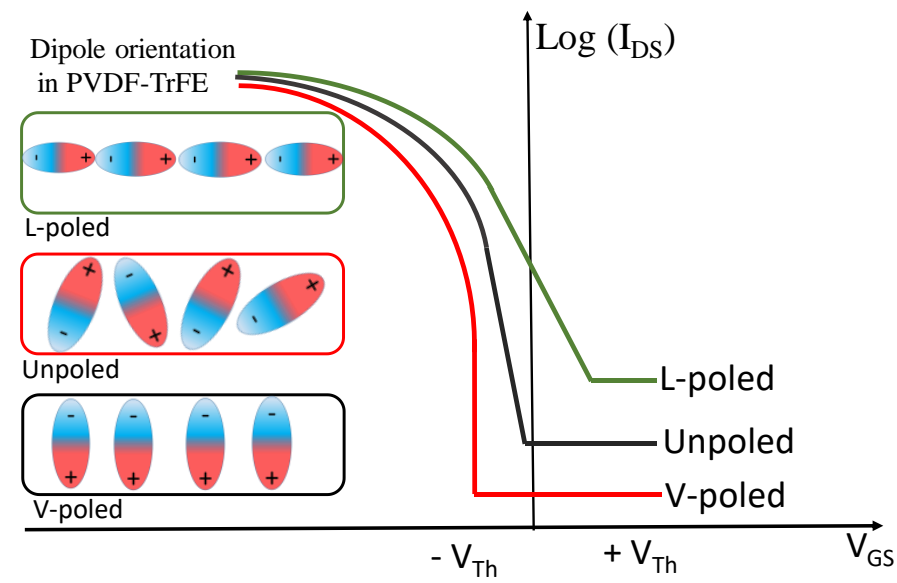

Figure 6.6 Schematic transfer curves of PVDF-TrFE-based FETs for different poling conditions. Vth may be positive or negative. A slightly positive Vth for the L-poled FET results in a higher current at zero gate bias condition depicted by the circle). The dipole orientation in PVDF-TrFE with poling conditions are also shown.

We note that the operating voltages of the FETs are well below the coercive fields; thus, no spontaneous polarization inversion is expected. The differences in the V-poled, unpoled, and L-poled FETs may be further understood in terms of $V_{\mathrm{Th}}$, as shown schematically in figure 6.6. The L-poled FET has a slightly positive $\mathrm{V}_{\text {Th }}$ (figure $6.2(\mathrm{~d})$ ). Hence, at zero gate voltage there is some current flowing between the source and drain electrodes, reducing both the on-off ratio and SS compared to the V-poled or unpoled devices. Although the effect of L-poling the V-poled device is not as dramatic as the initial L-poled sample, the trends are similar; $\mathrm{V}_{\mathrm{Th}}$ becomes less negative with L-poling (Table 6.II). It is worth pointing out that charge modulation reflectance measurements from 
pentacene ferroelectric FETs suggest a minority carrier injection near the source and drain electrodes, when the polarization direction is inverted (positively polarized at the interface). ${ }^{102}$ In addition to the changes in the interface potential with different poling conditions, one may ask the following questions in order to understand the dependence of the polarization rotation on the FET properties: does the microstructure of the PVDF-TrFE film change when it is V- or L-poled? Does the thickness of the dielectric medium play a role in the polarization reorientation process? Before looking at the structural changes in PVDF-TrFE upon poling, we comment on the $C$ - $V$ measurements from MIM structures. These measurements serve as an independent tool to understand how the charge accumulation at the interface may be enhanced by orienting the polarization.

\subsubsection{Capacitance-voltage characteristics of PVDF-TrFE metal-insulator- metal capacitors}

Figure 6.7 (a) shows the $C-V$ characteristics from Al-PVDF-TrFE-Au MIM structures for unpoled, V-poled, and L-poled films, measured at $5 \mathrm{kHz}$. All films had similar dielectric layer thicknesses $\sim 120 \mathrm{~nm}$. As expected from ferroelectric capacitors, PVDF-TrFE-based MIM capacitors exhibit a hysteresis with butterfly loops due to polarization reversal. ${ }^{103,104}$ The $\mathrm{C}-\mathrm{V}$ hysteresis indicates that the capacitance changes from an accumulation state to an inversion state. Clear differences are observed between the Vpoled, and the unpoled and L-poled films. The V-poled film has the highest value of capacitance; the L-poled film shows the highest asymmetry in the loop. The polarization switching occurs at the coercive voltage $(\mathrm{Vc})$; for the unpoled film $\mathrm{Vc} \sim 7 \mathrm{~V}$, similar to what is seen in the polarization versus voltage loop (figure 2.9). The dissipation factor, 
$\tan \delta$, is reasonably low $(\sim 0.03)$ between $\pm 15 \mathrm{~V}$ [figure. 6.7 (b)]. The $\mathrm{C}-\mathrm{V}$ measurements are further carried out from the V-poled MIM capacitor by applying a lateral voltage, shown in figure 6.7 (c). With an increase in the L-poling voltage, the overall accumulation capacitance decreases. These observations corroborate the FET characteristics; the SS value decreases with an increase in L-poling of the V-poled device as seen in figure 6.2 (e).
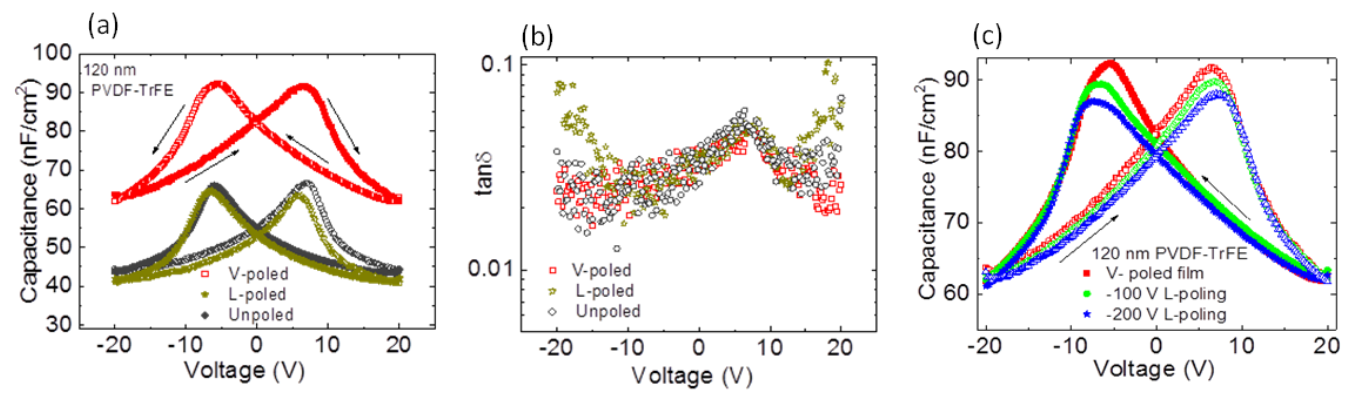

Figure 6.7 Capacitance - voltage characteristics of PVDF-TrFE MIM capacitors. (a) Capacitance versus voltage curves for V-poled, L-poled, and unpoled PVDF-TrFE films. The arrows show the direction of the applied voltage. (b) Corresponding tan $\delta$ values from the three films. (c) Capacitance versus voltage curves after L-poling of a V-poled film.

Polarization-rotation studies are further conducted on thinner PVDF-TrFE $(30 \mathrm{~nm})$ DNTT FETs. Results from the thinner PVDFTrFE films, as shown in figure 6.8 confirm two aspects. First, the operating voltages can be further reduced, well below $-2 \mathrm{~V}$, improving the overall transport properties in DNTT FETs. Second, it confirms that $\sim 120$ $\mathrm{nm}$ thickness is within the critical regime where polarization rotation is achievable, similar to the $30 \mathrm{~nm}$ devices. 
(a)

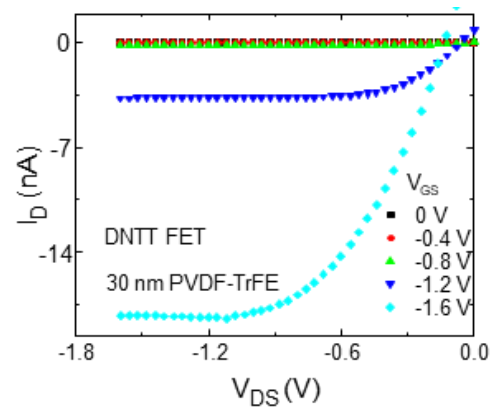

(b)

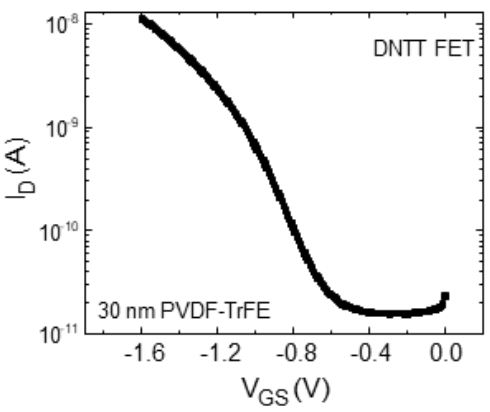

(c)

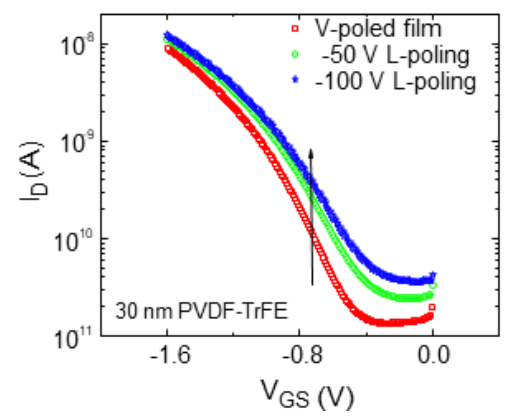

Figure 6.8 Typical FET characteristics of DNTT based devices with $30 \mathrm{~nm}$ PVDF-TrFE layer. (a) The voltage between source and drain was swept from 0 to $-1.6 \mathrm{~V}$ to obtain the output characteristics at various gate voltages varying from 0 to $-1.6 \mathrm{~V}$. (b) The transfer curve was swept in the saturation region where the drain-source voltage $\left(\mathrm{V}_{\mathrm{DS}}\right)$ was maintained at $-1.6 \mathrm{~V}$, and the gate voltage $\left(\mathrm{V}_{\mathrm{GS}}\right)$ was varied from 0 to $-1.6 \mathrm{~V}$. Both output and transfer curves were from a V-poled PVDF-TrFE based FET. (c) Transfer characteristics of a V-poled FET after applying two different lateral electric fields. The lateral field was applied such that the polarization direction of the dielectric is oriented parallel to the external source-drain electric field.

Irrespective of the organic semiconductor layer, our results show that the polarization orientation in the polymer ferroelectric layer directly impacts FET performance. In particular, SS of the FET transfer characteristics may be lowered by orienting $\mathbf{P}$ in a direction parallel to the applied gate electric field. This agrees well with some of the highest switching times that have been observed for polymer ferroelectric FETs when the interface layer is pre-biased in the perpendicular direction. ${ }^{83}$ In order to understand how the microstructure affects the overall polarization in the ferroelectric layer, we obtain SEM images and conduct XRD studies from poled and unpoled PVDF-TrFE films. To obtain a better signal-to-noise ratio, the sample thickness for the XRD measurements is higher than what is used in FETs. The PVDF-TrFE films are deposited on $\mathrm{Si}^{++}$with thickness $\sim 800 \mathrm{~nm}$. 


\subsubsection{Structure and morphology comparison at different poling conditions}

Figures 6.9 (a)-(c) show the SEM images from unpoled, V-poled, and L-poled PVDF-TrFE films. The L-poled sample is the same one as the V-poled film; a lateral field of $\sim 10^{5} \mathrm{~V} / \mathrm{m}$ is applied after obtaining the electron micrographs from the V-poled film. We note that the images are obtained with a low accelerating voltage of the electron beam and in situ nitrogen plasma cleaning of the thin film surface in order to avoid any radiation damage or surface contamination due to residual hydrocarbons. Upon V-poling, the microstructure is seen to change compared to the unpoled sample; clear domains $>1 \mu \mathrm{m}$ are seen to form. Upon L-poling the same (V-poled) sample, the large domains are seen to diminish, and the sample morphology becomes similar to the unpoled sample. These results indicate that a much smaller electric field in the lateral direction compared to the electric field in the vertical direction affects the microstructure, which most likely changes the ferroelectric domains.

Figure 6.9 (d) shows the XRD data from unpoled, V-poled, and L-poled PVDFTrFE films. In each case, the Si background is subtracted. The absolute intensities in figure 6.9 (d) for the three samples are comparable as the thicknesses are similar. The poling condition for the L-poled film is similar to that used in Fig. 6.2 (b), where the film is heated along with the application of a lateral field of $\sim 10^{5} \mathrm{~V} / \mathrm{m}$.

The V-poled film is further L-poled using the same condition as in FETs. The XRD data from the same film for these two poling conditions are shown in the inset. The peak at $2 \theta$ of $\sim 20^{\circ}$ is from the (200) or (110) reflections, revealing the $\beta$ phase structure. ${ }^{105}$ The (hkl) indexing of (001) and (400/220) of the XRD Bragg peaks in figure 6.9 (d) is based 
on the pseudohexagonal nature of the ferroelectric $(\mathrm{FE})$ phase. ${ }^{106}$ The V-poled sample shows the strongest intensity for the (200) or (110) reflection, suggesting an enhancement of the overall $\beta$ phase. The L-poled and unpoled samples are almost identical except for a shoulder peak at $\sim 19^{\circ}$, which is more pronounced for the L-poled sample. This shoulder is most likely a signature of the $\alpha$ and $\gamma$ phases of the paraelectric (PE) phase $^{107}$; the deconvolution of the spectrum as two Gaussian peaks is shown in figure 6.9 (e).

The enhanced intensity of the $\beta$ phase diffraction in PVDF-TrFE upon V-poling may be understood from previous XRD measurements in PVDF. The increase in polarization of a PVDF film (upon poling) originates from reorientation of the dipoles in the crystalline phase by an electric field rather than orientation of the dipoles of the amorphous (PE) phase. ${ }^{108-110}$ It is pointed out that although the $\beta$ phase unit cell is orthorhombic, it is very close to being hexagonal. ${ }^{111}$ A small distortion of the primitive hexagonal unit cell is required to yield the $\beta$ phase unit cell. A slight change in the peak position of the (200) or (110) reflection (of the $\beta$ phase) upon V-poling compared to the unpoled sample in figure 6.9 (d) may reflect a unit cell closer to the $\beta$ phase unit cell. Due to the overlapping reflections, it is difficult to predict the exact lattice constants from our experiments. 
(a)
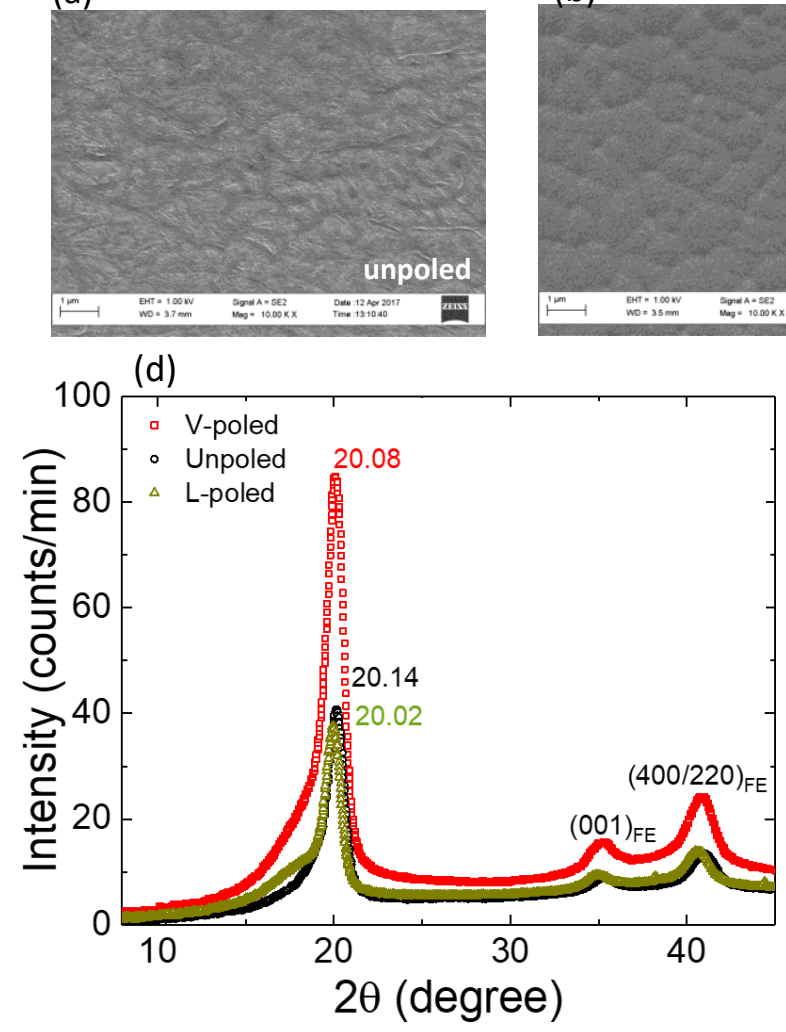

(b)

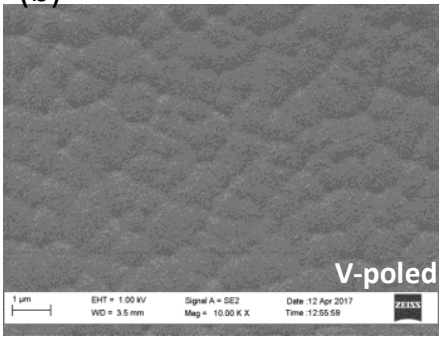

(c)

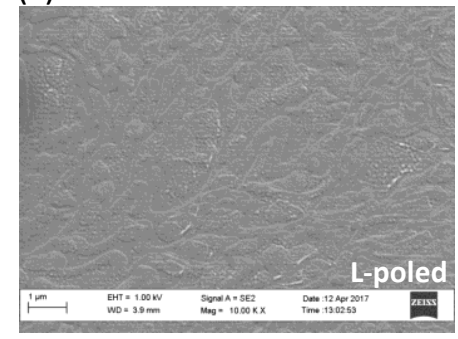

(e)

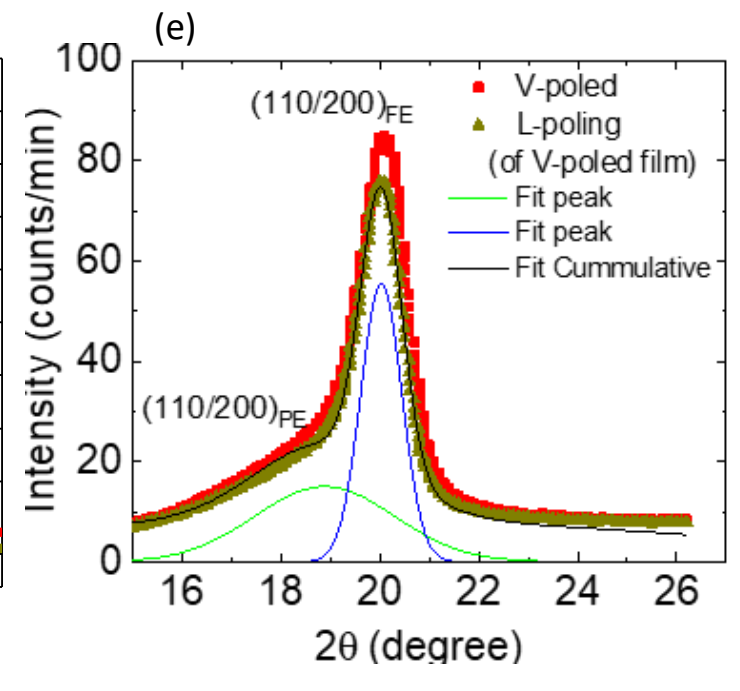

Figure 6.9 Structure and morphology of PVDF-TrFE as a function of poling. (a)-(c) Scanning electron micrographs from unpoled, V-poled, and laterally L-poled films. (d) Grazing-incidence XRD from unpoled, V-poled, and Lpoled PVDF-TrFE films on Si substrate. The (hkl) indexing is based on the ferroelectric phase as discussed in the text. (e) The XRD from the V-poled film and after L-poling the same V-poled film. Deconvolution of two peaks at $2 \theta(\mathrm{deg})\left(\beta\right.$ phase) and at $18^{\circ}$ for the paraelectric phase (PE) are shown for the laterally poled film.

Since the sample thickness of PVDF-TrFE for XRD measurements is about eight times more than those used in FETs, the polarization changes for the L-poled sample may even be higher in the FET devices compared to what is observed in Fig. 6.9(d). A small change in the XRD intensity after L-poling the V-poled film (in the inset) reflects a reorientation of the dipole moment of the unit cell that affects the $\beta$ phase. However, this change is much smaller compared to the L-poled film, which is poled during 
recrystallization. Similar to the XRD results, the FET results show a larger difference in SS for the PVDF-TrFE film, which is L-poled during recrystallization compared to Lpoling a V-poled FET (Tables 6-I and 6-II).

A simple molecular model is used to understand the role of the electric field on the dipole moment of a PVDF-TrFE molecule. Although in the crystalline environment, the mechanism of polarization rotation may be quite different, application of external fields to the molecule reveals changes in the all-trans configuration. DFT optimization of a PVDFTrFE molecule in a solvent atmosphere is shown in figure 6.10.

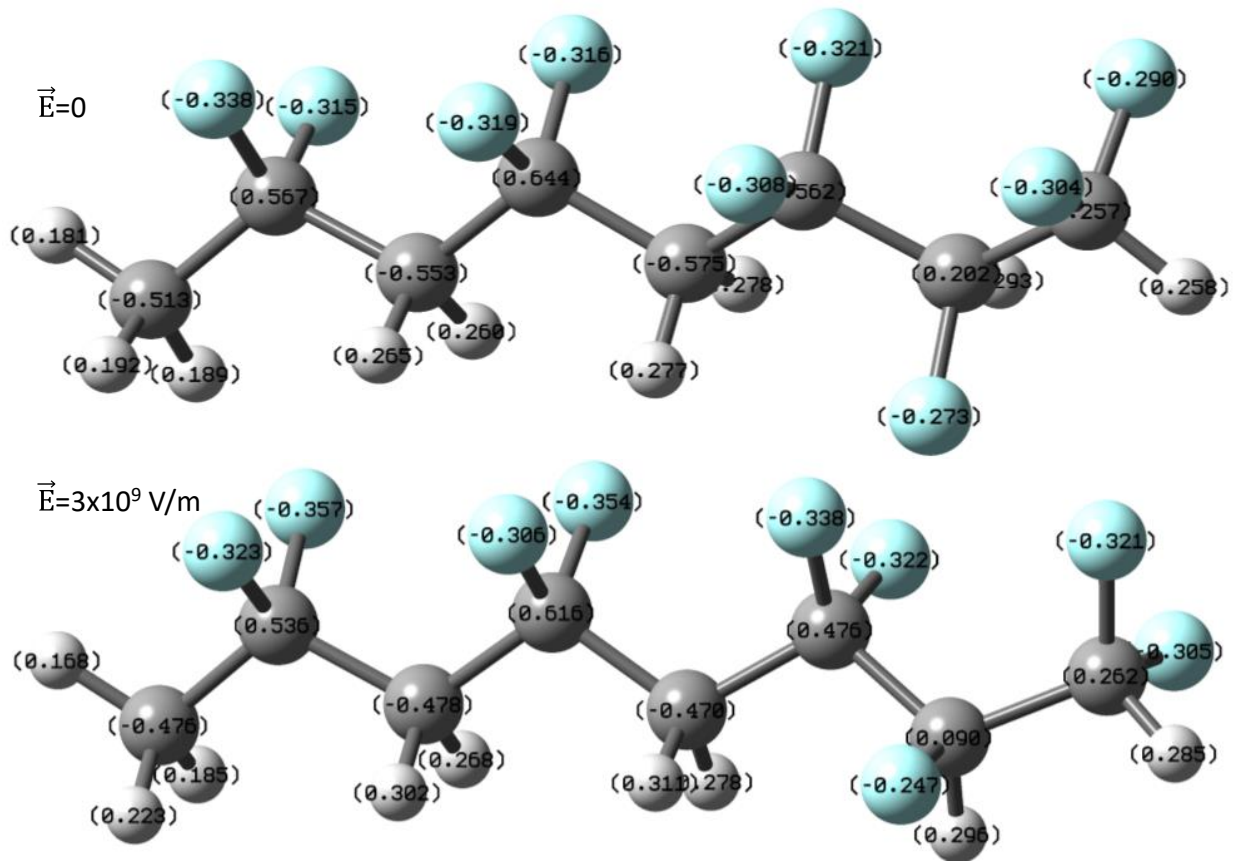

Figure 6.10 DFT optimization of a PVDF-TrFE molecule in a solvent atmosphere. The charge distribution with no external field (top) and in the presence of an external field applied perpendicular to the chain axis (bottom). One unit of PVDF-TrFE (3:1) molecule was optimized in the presence of DMF solvent with no external field. The optimized molecule was again optimized with the applied electric field. 
The model was performed in collaboration with Dr. Soma Khanra. As shown in Fig. 6.11(a), a single unit of the molecule is first optimized in a solvent bath. Since fluorine is more electronegative than hydrogen and carbon, the dipole moment points away from fluorine towards the hydrogen atoms (blue arrow). Electric fields of different magnitudes are applied in a perpendicular direction [figure 6.11 (a-b]. A lateral field in the opposite direction makes the molecule more linear but decreases the overall dipole moment (blue arrow) compared to the starting condition. The Table 6-III shows a decrease in the dipole moment upon the application of a lateral field (of $5 \times 10^{8} \mathrm{~V} / \mathrm{m}$ ). The higher fields (above $5 \times 10^{9} \mathrm{~V} / \mathrm{m}$ ) increase the dipole moment but in the parallel direction.

Table 6-III Dipole moment of a PVDF-TrFE (in a solvent bath) upon application of an external electric field in the lateral direction.

\begin{tabular}{cc}
\hline Electric Field $(\mathrm{V} / \mathrm{m})$ & Dipole Moment (Debye) \\
\hline Vertically poled & 12.963 \\
$0.514 \times 10^{9}$ & 10.669 \\
$2.06 \times 10^{9}$ & 10.665 \\
$3.09 \times 10^{9}$ & 10.749 \\
$4.14 \times 10^{9}$ & 11.082 \\
\hline
\end{tabular}

When the field is applied in perpendicular (vertical) direction, the dipole moment decreases as shown in the Table 6-IV suggesting the vertical poling is enhancing the dipole moment of the dielectric. 
Table 6-IV Dipole moment of a PVDF-TrFE (in a solvent bath) upon application of an external electric field in the perpendicular direction.

\begin{tabular}{cc}
\hline Electric Field $(\mathrm{V} / \mathrm{m})$ & Dipole Moment (Debye) \\
\hline 0 & 10.008 \\
$1.03 \times 10^{9}$ & 11.265 \\
$2.06 \times 10^{9}$ & 12.269 \\
$3.09 \times 10^{9}$ & 12.963 \\
\hline
\end{tabular}

Since the molecule is embedded in a solvent, the magnitude of the field in the DFT simulations is a few orders of magnitude higher compared to experiment. The dipole moment increases from $10 \mathrm{D}$ (at zero external field) to almost $13 \mathrm{D}$ after applying a maximum field of $3 \times 10^{9} \mathrm{~V} / \mathrm{m}^{101}$

Applying a lateral field reorients the dipole moment in the horizontal direction and results in an overall buckling of the molecule [figure 6.11 (d)] with a deviation from the all-trans phase. To mimic the reversal of the field in FET measurements, we reverse the direction of the applied lateral field [figure $6.11(\mathrm{e})$ ]. The molecule becomes linear but it does not recover to its initial all-trans phase (for the magnitude of the field shown). These model calculations serve as a guide to correlate the changes in FET properties upon $\mathrm{V}$ - and L-poling for different magnitudes of applied fields. Application of a lateral electric field to the PVDF-TrFE film reorients the polarization in the horizontal direction such that it diminishes the screening charge accumulation, increasing SS and degrading FET properties. The simulations show that electric fields smaller by an order of magnitude in the lateral direction compared to the vertical direction can dramatically change the dipole moment. This result is similar to our experimental observation where a lateral field, which 
is two or three orders of magnitude smaller compared to the vertical field, can change SS by almost $40 \%$.

(a)

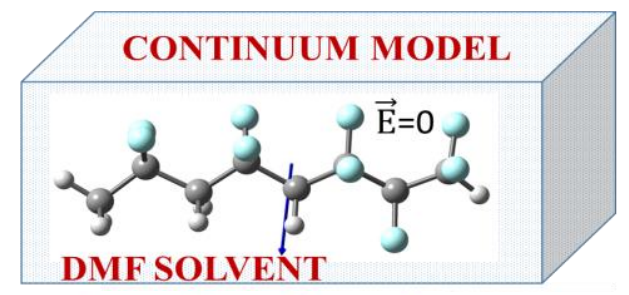

(b)

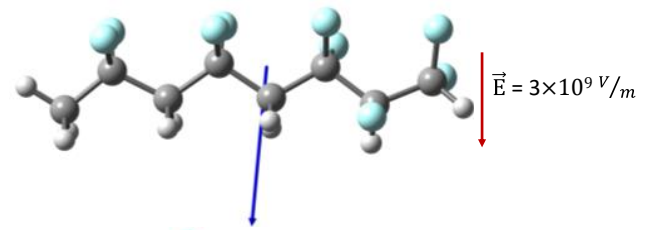

(c)

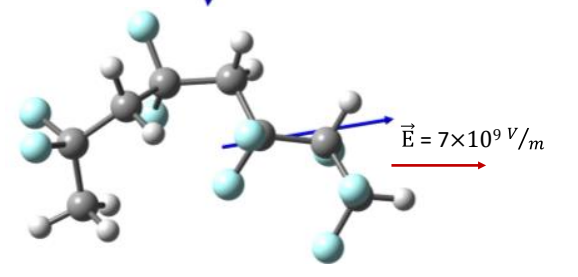

(d)

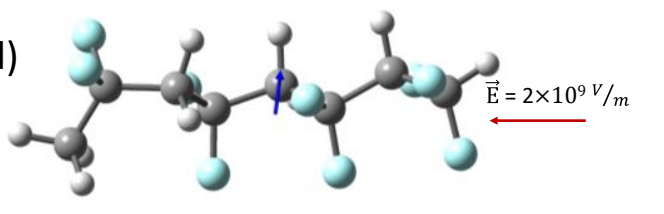

Figure 6.11 (a) DFT calculations of the structural changes on a PVDF-TrFE oligomer enclosed in a dielectric medium upon the application of an external electric field. Blue arrows indicate the direction of the dipole moment. (b-d) Structural changes of PVDF-TrFE after the application of a lateral electric field with various magnitude and directions. The application of a lateral field buckles the molecule (representative of a combination of the $\alpha$ and $\delta$ phase), shown in the middle.

In summary, polarization rotation in a relaxor ferroelectric dielectric, PVDF-TrFE, has a profound effect on the transport properties of organic FETs. The overall FET properties are enhanced when the ferroelectric layer is vertically poled such as SS decreases and on/off ratio increases. This result is independent of semiconductors. Such an external 
tuning knob not only benefits an understanding of charge-transport mechanisms in a wide range of polymeric and molecular semiconductor-based devices but also provides new design architectures for improving FET performance. By employing low-operating voltage FETs, we independently exploit the impact of polarization rotation on the FET characteristics. A caveat for high-operating voltage FETs is that L-poled FETs may behave like V-poled devices due to polarization inversion in the dielectric layer by the gate voltage during normal FET operation. A strong effect of polarization modulation may be envisioned if the transistor architecture is scaled down by reducing channel lengths, decreasing the distance between the lateral electrodes, and by using thin ferroelectric dielectric films. Channel lengths in the tens to hundreds of nm range and a thin dielectric layer (tens of $\mathrm{nm}$ ) lowers the operating voltage of FETs. Such dimensions may allow poling of the dielectric layer during the normal operation of the FET, resulting in SS values close to that of MOSFETs.

A small channel length, which may be achieved by nanolithography, allows the placement of the lateral electrodes closer than the $\mathrm{mm}$ distances used in this work. The energy needed for polarization rotation will thus be reduced, and one may expect larger differences in SS and other FET transport properties between V-poled and L-poled devices. Orienting and reorienting the polarization direction serves as a tuning parameter for manipulating SS and carrier mobilities in organic FETs. L-poling of a V-poled PVDF-TrFE film shows changes in carrier transport in FETs as well as reduces the overall capacitance in MIM structures. SEM images and XRD from poled and unpoled PVDF-TrFE reveal subtle changes in the microstructure and overall polarization of the PVDF-TrFE film. DFT calculations of the dipole moment from a molecular unit embedded in a solvent bath show 
anisotropy in the poling direction; a smaller electric field in the lateral direction (along the backbone of the molecule) compared to the perpendicular direction results in a large change in the dipole moment. This study highlights new design principles for organic FETs in lowering SS and enhancing transport properties by orienting the polarization direction in the ferroelectric dielectric layer.

\subsection{Textured poling of dielectric PVDF-TrFE}

Section 6.1 highlighted the impact of rotating the polarization direction of PVDF-TrFE by $90^{\circ}$ on the FET properties of small molecule semiconductors. ${ }^{101}$ Lateral poling of the dielectric layer from the top surface in bottom-gate, top-contact FET geometries degrades the FET characteristics. The overall transistor properties are enhanced when the ferroelectric layer is vertically poled, and are easily and reversibly controlled by switching the polarization direction. Other studies revealed that electrical poling of the dielectric layer is an effective means of tuning transport properties in organic FETs. ${ }^{91,92}$ The question we ask here is whether there is a judicious way of external poling the ferroelectric layer by incorporating both vertical and lateral polarization of the dielectric layer to reduce the gate leakage current, increase on/off ratios, and improve the mobility of charge carriers in organic FETs using > $100 \mathrm{~nm}$ thick dielectric films, and yet have them operate at low voltages.

\subsubsection{Technique used for textured poling of PVDF-TrFE and FET performance}

By using solution processed TIPS-pentacene as the organic semiconductor, we show that textured poling of the PVDF-TrFE layer dramatically improves FET properties 
compared to unpoled or uniformly poled ferroelectric films. Prior to the deposition of the organic semiconductor layer, the poling of the gate ferroelectric dielectric (PVDF-TrFE) is achieved by applying an external electric field while crystallization of the film occurs just past its ferroelectric-paraelectric transition temperature. Vertical poling is achieved as we discussed in section 6.1.1. Details of work can be found in Ref. ${ }^{112}$
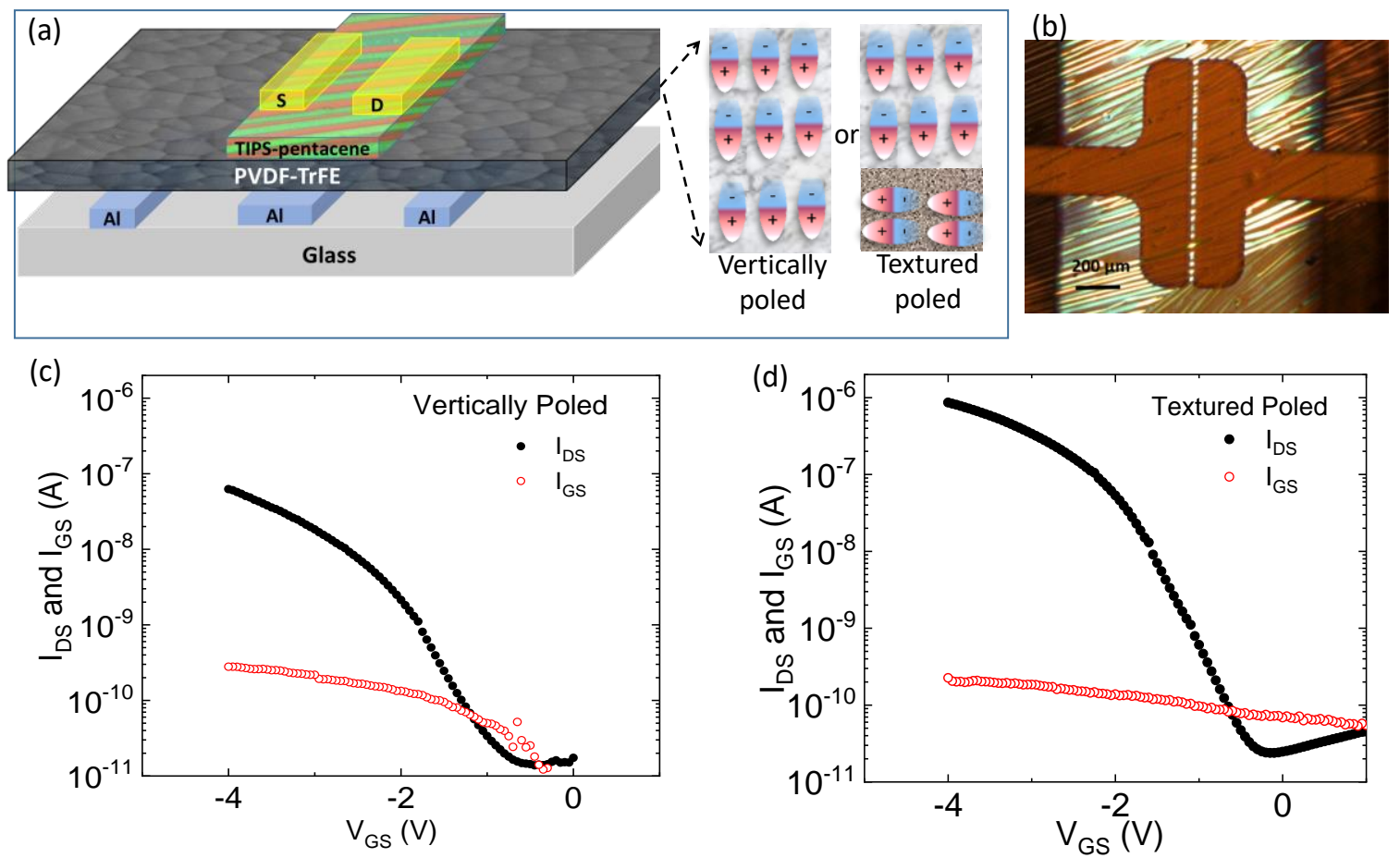

Figure 6.12 Textured poling in PVDF-TrFE films for application in TIPS-pentacene FETs. (a) Schematic representation of the FET geometry with three electrodes at the bottom. The two lateral Al strips serve as electrodes for applying the lateral voltage after the PVDF-TrFE film was vertically poled. Schematics of vertical and textured poling are represented. (b) Optical image of a bottom-gate top-contact TIPS pentacene FET. (c) Transfer characteristics from a vertically poled PVDF-TrFE/TIPS-pentacene device. The gate leakage current is shown in red. (d) Transfer characteristics from a textured poled PVDF-TrFE/TIPSpentacene device. The gate leakage current is shown in red. 
The PVDF-TrFE films were grown on substrates that were equipped with two lateral electrodes flanking the gate electrode, as schematically shown in figure 6.12 (a). After vertical poling, a lateral field could be applied from the underside of the PVDF-TrFE films. This ensures that the lateral field only penetrates a part of the dielectric film from the bottom. We refer to textured poling in this work as a combination of first vertically poling the PVDF-TrFE layer and then applying a lateral field for a short time from the bottom of the dielectric layer The textured poling yields high on/off current ratios by reducing the gate leakage current and, in general, improves the overall FET properties. This study opens the prospect of achieving low-operating, high-performance, low-cost organic FETs by a textured polarization of the ferroelectric dielectric without any expensive patterning techniques.

Figure 6.12 (c) and (d) show transfer sweeps from vertically poled and textured poled PVDF-TrFE based TIPS-pentacene FETs, respectively. The lateral field (for textured poling) was $10^{4} \mathrm{~V} / \mathrm{m}$. One clearly sees an improvement in the on current and a relative reduction in the vertical gate-source current upon textured poling. Figure 6.13 shows the transfer sweeps with $\sqrt{I_{D S}}$ to extract the mobilities for unpoled and V-poled PVDF-TrFE FETs. 

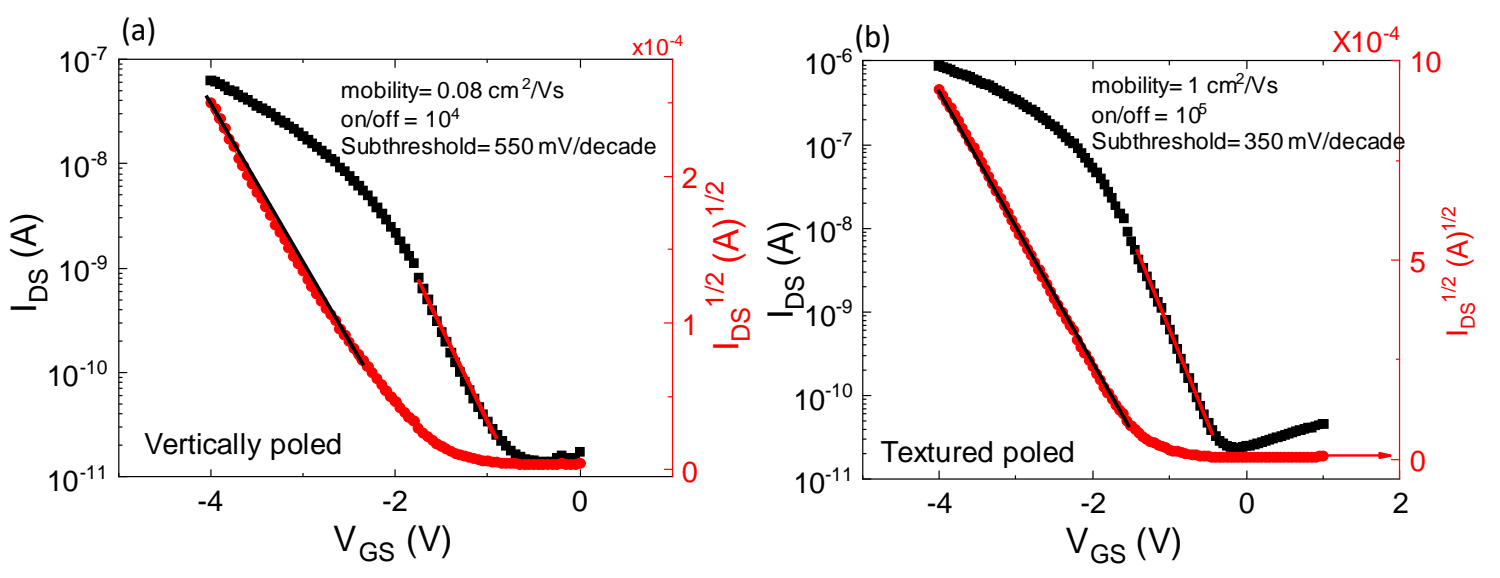

Figure 6.13 Typical transfer and square root of current plots to show how the saturation mobility and subthreshold swing (SS) parameters were extracted. (a) Transfer characteristics and square root of $\mathrm{I}_{\mathrm{DS}}$ of a vertically poled PVDF-TrFE/TIPS-pentacene FET. (b) Transfer characteristics and square root of IDS of a textured poled PVDF-TrFE/TIPS-pentacene FET.

The best performing textured poled FETs yield mobility of $1 \mathrm{~cm}^{2} / \mathrm{Vs}$ or higher, on/off ratio of $10^{5}$ and a SS of $200 \mathrm{mV} /$ decade whereas the vertically poled FETs yield mobility $0.08 \mathrm{~cm}^{2} / \mathrm{Vs}$, on/off ratio of $10^{4}$ and a $\mathrm{SS}$ of $350 \mathrm{mV} /$ decade. On average, the carrier mobilities are seen to improve by an order of a magnitude for the textured poled devices. 

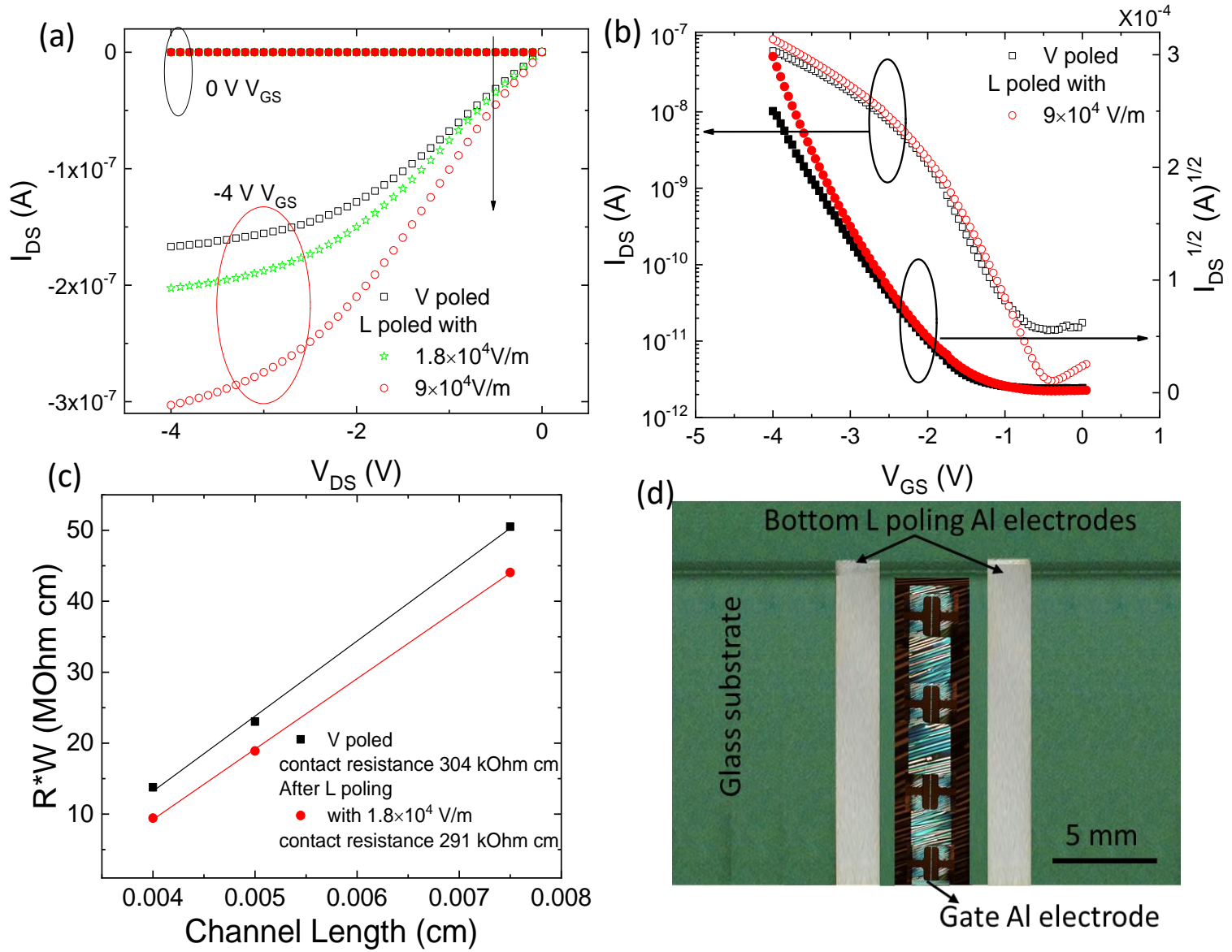

Figure 6.14 TIPS-pentacene FET characteristics for V poled and textured poled PVDF-TrFE films. (a) Output characteristics of a TIPS-pentacene FET with V poled PVDF-TrFE (black squares). The same device was then L poled from the bottom for various magnitudes of electric field; output characteristics of the same FET for two such L poling conditions. (b) Transfer characteristics from the V poled PVDF-TrFE/TIPSpentacene device and the same device after $\mathrm{L}$ poling from the bottom (with a field of $9 \times 10^{4} \mathrm{~V} / \mathrm{m}$ ). Both $\mathrm{I}_{\mathrm{DS}}$ and the square root of $I_{D S}$ as a function of the gate voltage are shown. The drain-source voltage was held at $-4 \mathrm{~V}$. (c) The product of the total resistance and the channel width (W) versus channel length (L) for three different values of W/L for V poled (black square) and textured poled (red circle) TIPS-pentacene FETs. (d) Optical image of a substrate containing the TIPS-pentacene FETs along with the lateral electrodes that are placed below the PVDF-TrFE layer. 
Figure 6.14 (a) shows the output characteristics of a V-poled FET at $V_{G S}$ of $0 \mathrm{~V}$ and 4V (black square). The same device was then L-poled for different magnitudes of electric fields for $15 \mathrm{~min}$, and then the FET characteristics were measured. The results after applying field strengths of $1.8 \times 10^{4} \mathrm{~V} / \mathrm{m}$ and $9 \times 10^{4} \mathrm{~V} / \mathrm{m}$ are shown. An improvement in the FET modulation in the output characteristics is seen after L poling. The transfer curves from the same V poled FET (as in figure 6.14 (a)) and after L poling the device is shown in Figure 6.14 (b). The off current $\left(\mathrm{I}_{\mathrm{DS}}\right)$ goes down after L poling along and shows a higher slope for $\sqrt{I_{D S}}$ compared to the only $\mathrm{V}$ poled FET.

One may ask whether the improvement in FET characteristics after L poling the Vpoled device (textured poling) is related to a change in the contact resistance. Each substrate supported several FETs with varying W/L ratio as shown in figure 6.14 (d). Contact resistance normalized with width of the channel (W) is obtained by using the transmission line method (TLM), commonly used for the calculation of contact resistance in FETs. ${ }^{113,114}$

$$
\mathrm{RW}=\mathrm{R}_{\mathrm{C}} \mathrm{W}+\frac{\mathrm{L}}{\mu \mathrm{C}_{\mathrm{i}}\left(\mathrm{V}_{\mathrm{GS}}-\mathrm{V}_{\mathrm{Th}}\right)}
$$

Where $\mathrm{R}$ is total resistance, $\mathrm{R}_{\mathrm{C}}$ is contact resistance, $\mathrm{C}_{\mathrm{i}}$ is the dielectric capacitance, $\mu$ is the carrier mobility, $\mathrm{L}$ is the channel length, $\mathrm{V}_{\mathrm{GS}}$ and $\mathrm{V}_{\mathrm{Th}}$ are the gate-source and threshold voltages, respectively. The product of the total resistance and the channel width versus channel length for three different values of W/L for V-poled (black square) and L poled with $1.8 \times 10^{4} \mathrm{~V} / \mathrm{m}$ (red circle) are shown in figure 6.14 (c). The contact resistance $(\sim 300$ $\mathrm{k} \Omega \mathrm{cm}$ ) is approximately the same for both conditions of poling, and is at least two orders of magnitude smaller than the channel resistance. Thus, the differences in the $\mathrm{V}$ poled and 
the textured poled devices arise from an improvement in the dielectric layer itself. It is not surprising that the contact resistance of TIPS-pentacene FETs here is higher with PVDFTrFE compared with other dielectrics found in the literature; it is mainly due to the effect of polarization fluctuation at the semiconductor-dielectric interface.

\subsubsection{Poling field optimization, microscopy and simulation}

Next, we look at optimum lateral electric fields required for improving the FET characteristics. As was shown in Ref. ${ }^{101}$, lateral poling from the top of the dielectric layer induces a spontaneous parallel electric field, which greatly increases the off current and degrades SS and $\mu$. One may expect a similar scenario by L poling from the bottom; if the electric field is high enough, it may penetrate the bulk of the dielectric layer. Figure 6.15 (a) shows the transfer characteristics for a $\mathrm{V}$ poled and after $\mathrm{L}$ poling from bottom by applying an electric field of $10^{5} \mathrm{~V} / \mathrm{m}$. Here we clearly see a degradation of transfer characteristics where the off current is greatly enhanced and $\mathrm{V}_{\mathrm{Th}}$ is seen to become positive. What may seem a bit odd at the first glance is the difference between figure 6.15 (a) and figure 6.15 (b) as the magnitude of the lateral fields are not that different. We point out that the field distribution here is not uniform. As there is the central gate electrode, the penetrated field can be quite different at different locations. One expects from Gauss's law that right in the middle of the central gate electrode, the electric field is exactly zero. Hence, if the FETs are slightly displaced from the center of the gate electrode (bright central region shown in figure $6.15(\mathrm{~d})$ ), the field distribution will be higher. We point out that the FET in figure 6.15 (a) was more off-centered compared to the device shown in figure 6.14. As a comparison, we show the transfer characteristics of another V poled FET where it was 
poled from the top (with an order of magnitude smaller field compared to L poling from the bottom) in figure 6.15 (b). The off current goes up and the overall FET properties degrade upon lateral poling from the top.

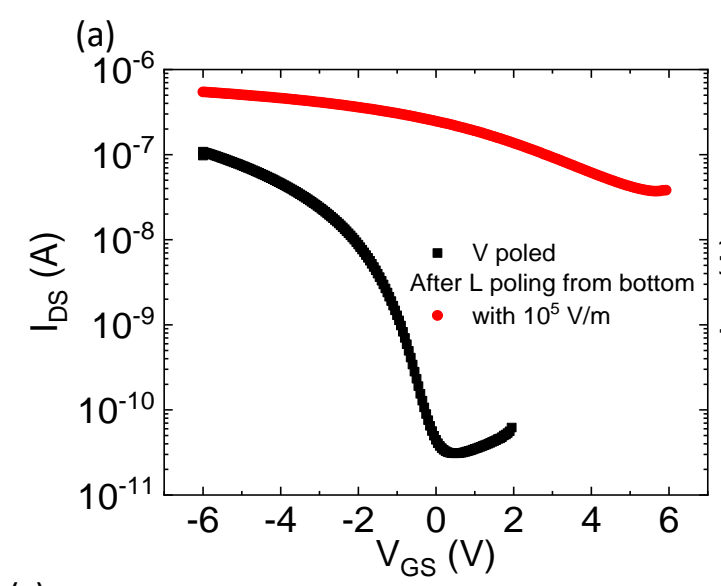

(c)

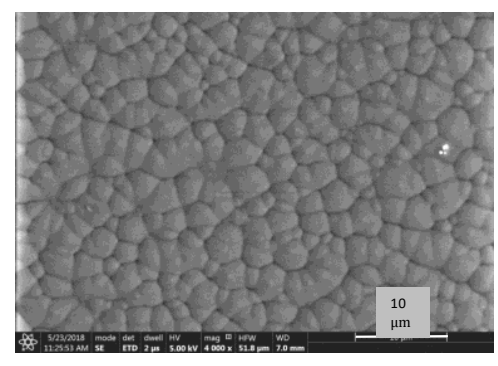

(b)

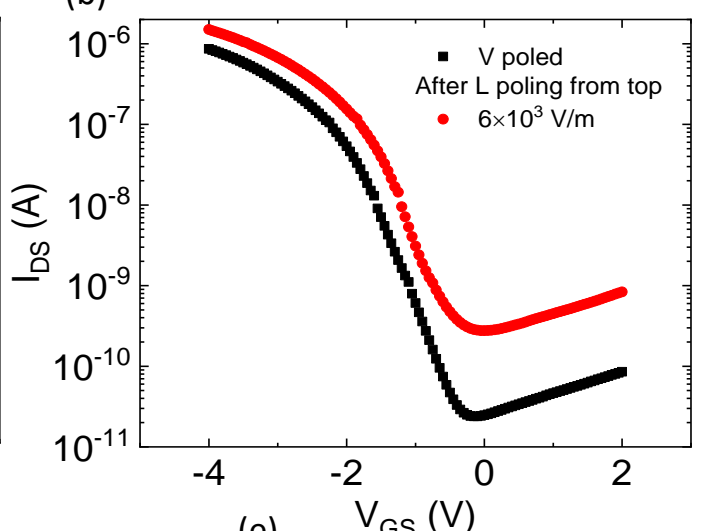

(e) $V_{G S}(V)$

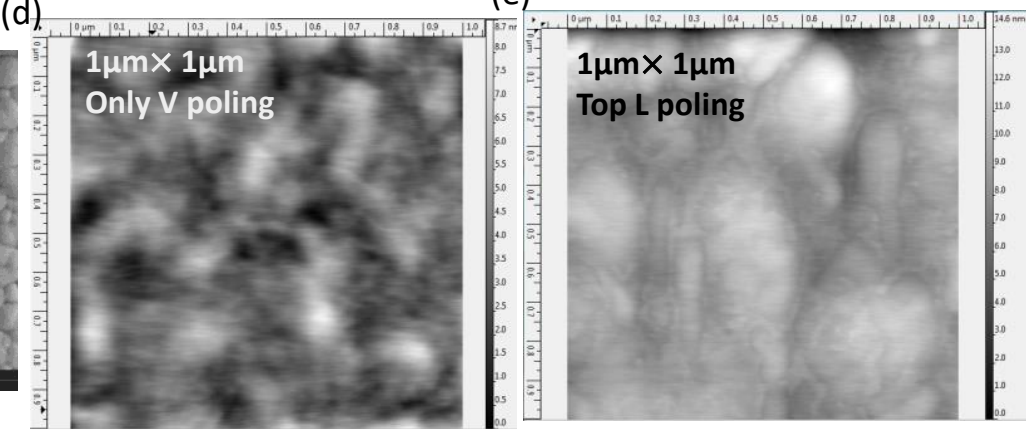

Figure 6.15 Transfer characteristics of TIPS-pentacene FETs with different poling conditions of the PVDFTrFE layer and surface morphology. (a) Transfer characteristics of a V-poled PVDF-TrFE layer (black) and the transfer curve for the same device after L-poling (from the bottom) using an electric field of $10^{5} \mathrm{~V} / \mathrm{m}$ (red). (b) Transfer curve of a V-poled FET (black) and after L-poling the same device from the top with an electric field of $6 \times 10^{3} \mathrm{~V} / \mathrm{m}$ (red). (c) SEM image of a V-poled PVDF-TrFE film. (d) AFM image of a V poled PVDF-TrFE film. (e) AFM image of a V-poled PVDF-TrFE film, which was L-poled from the top with an electric field of $6 \times 10^{3} \mathrm{~V} / \mathrm{m}$.

SEM images ( figure 6.15 (c)) show that V-poled PVDF-TrFE films show large domains $>1 \mu \mathrm{m}$ as shown in the. L poling from the bottom does not change the top microstructure, and the morphology at the TIPS-pentacene-dielectric interface remains 123 
unchanged. These features are discernable even with AFM images at small length scales. Figure 6.15 (d) shows $1 \mu \mathrm{m} \times 1 \mu \mathrm{m}$ AFM image of a film that was V-poled which is similar to films that are L-poled from the bottom. Upon L poling from the top, the morphology of the film is seen to change along with the increase in the off currents. In this work the processing conditions for depositing TIPS-pentacene was not changed; only the poling conditions of the dielectric were altered.

We obtained cross-sectional TEM images from V-poled, textured poled and unpoled PVDF-TrFE films grown on Al coated glass. A focused ion beam (FIB) SEM dual beam instrument was used for creating a cross-section from the sample, which was subsequently lifted out of the sample. TEM cross-sections were prepared in the Scios DualBeam via the $\mathrm{Ga}$ ion beam. First, between $0.5-1 \mu \mathrm{m}$ of Pt was deposited using the electron beam, followed by $2 \mu \mathrm{m}$ of Pt deposited using the ion beam. The sample was then trenched to form a cross-section and cut away from the substrate using the ion beam at $30 \mathrm{kV}$ and various beam currents. The cross-sections were subsequently lifted out when attached to a needle within the Scios, and polished from $2 \mu \mathrm{m}$ to $100 \mathrm{~nm}$ using smaller ion beam currents and voltages as the cross-sections became thinner. Finally, a Ga ion beam with low $\mathrm{kV}$ and low current $(2 \mathrm{kV}, 15 \mathrm{pA})$ was employed to further clean off the surface amorphorization layer. The cross-sections were attached to a copper omniprobe through Pt deposition, which were then able to be directly loaded onto the TEM single-tilt holder. HRTEM imaging was conducted in a FEI Tecnai F30 G2 at $100 \mathrm{kV}$. Beam alignments were conducted on the glass portion of the cross-sections to minimize beam damage and any interactions with the PVDF-TrFE film. 
Figure 6.16 (b)-(e) show the cross-sectional TEM images from textured and unpoled films grown on Al coated glass, identical to the FETs. The textured poling was achieved as described earlier. Overall, the bulk of the PVDF-TrFE morphology looks quite similar for poled and unpoled films. Since the Al layer is also lifted out along with the PVDF-TrFE, there is an issue of additional poling by the e-beam while imaging near the Al interface over time. Even then, we find subtle differences between the textured poled and unpoled films. For the textured poled sample, there is a difference in the morphology of the PVDF-TrFE film near the Al electrode compared to the bulk as shown in figure 6.16 (b). The initial TEM images show a layer of approximately $30-40 \mathrm{~nm}$ region near the electrode with a different morphology, which is lighter in color (highlighted by the blue box). Upon subsequent measurements, this layer expands from $40 \mathrm{~nm}$ to $55 \mathrm{~nm}$ due to additional polarization by the e-beam in the proximity of the $\mathrm{Al}$ interface (figure 6.16 (c)). It is important to note that this narrow layer near the Al electrode is immediately visible in textured poled TEM images. In contrast, the unpoled sample does not show any difference in morphology throughout the PVDF-TrFE film in the first few TEM images (figure 6.16 (d)) but over time there is a layer near the $\mathrm{Al}$ electrode that looks quite similar to the textured poled films (figure 6.16 (e)). The TEM measurements yield some insight into the penetration of the field into the dielectric layer upon textured poling; however, it is difficult to systematically quantify the extent of lateral poling in the dielectric layer as a function of the electric field due to additional poling problems. Since textured poling is achieved by lateral poling after vertical poling, the bottom Al electrode is necessary. 
(a)

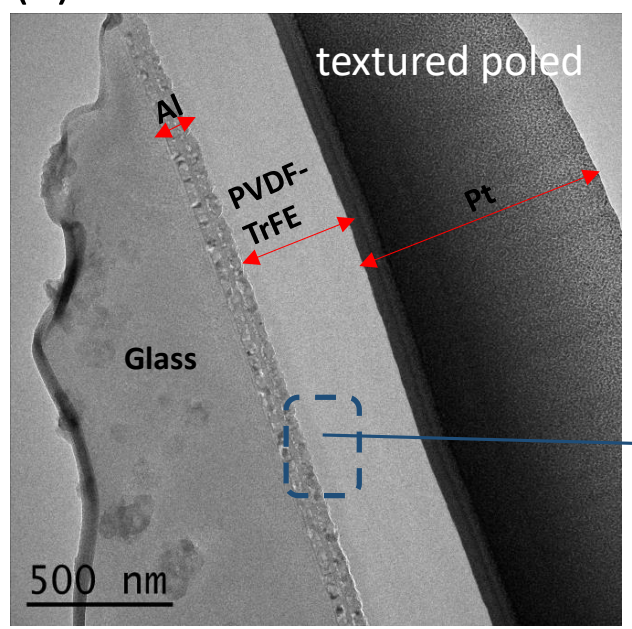

(c)

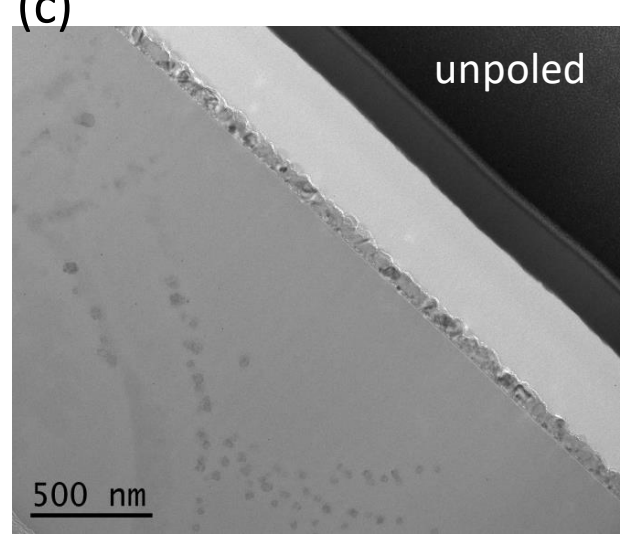

(b)

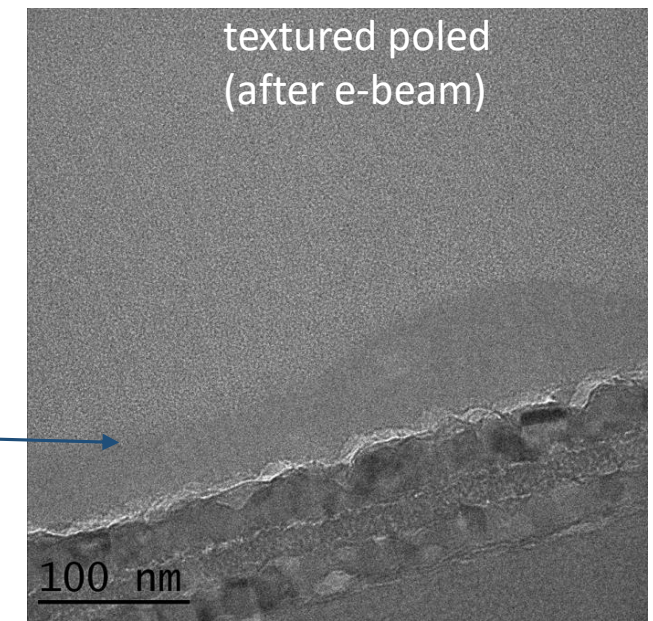

(d)

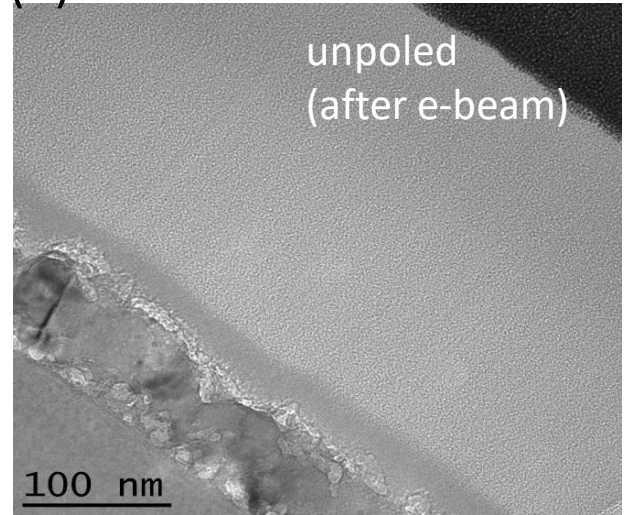

Figure 6.16 Cross-sectional TEM images of poled and unpoled PVDF-TrFE layer. (a) and (b) Cross-sectional TEM images from a textured poled PVDF-TrFE film. The PVDF-TrFE films of thickness 300 nm was deposited on Al coated glass. The image in (a) shows a slightly different shading near Al due to lateral poling. In (b), this region near the electrode is seen to grow due to additional polarization during the measurements. (c) and (d) Cross-sectional TEM images from an unpoled PVDF-TrFE film. The PVDF-TrFE films looks smooth and no different shading is seen near the Al electrode at the onset in (c). Due to additional polarization, a different shading occurs for $\sim 20 \mathrm{~nm}$ of the film near the Al electrode after a few measurements.

Synopsys Sentaurus (physics-based technology computer aided design (TCAD) Simulations were performed to model the electric field formation in the ferroelectric 
dielectric layer. Sentaurus Device has an extensive set of device physics models and is capable of modeling drift-diffusion, thermodynamic, and hydrodynamic effects in semiconductor devices. It enables the numeric simulation of electrical behavior at user defined mesh points using coupled Poisson equation. The meshing scheme for the simulated device stack, using TCAD, is shown in figure 6.17 (a-b).

The 2-D structure model consisted of $500 \mu \mathrm{m}$ thick glass substrate with $400 \mathrm{~nm}$ thick dielectric layer. Aluminum source and drain electrodes ( $25 \mu \mathrm{m}$ wide, $60 \mathrm{~nm}$ thick) were separated by $550 \mu \mathrm{m}$ gap. In the center of the device was an unbiased gate contact of the same dimensions as the source-drain electrode. The dielectric material parameters were modeled using the generic Insulator2 material file in the Sentaurus suite. The dielectric constant of this material was changed to 8.3 to better simulate real device. The device was biased from source to drain using a quasistationary voltage ramp to $20 \mathrm{~V}$ with minimum step size of $10^{-5} \mathrm{~V}$ and maximum step size $0.1 \mathrm{~V}$, solving the coupled Poisson electron-hole formula at each step. The mesh was refined to $100 \mathrm{~nm}$ x $100 \mathrm{~nm}$ around the length of the dielectric/Al contacts (refer to Fig. S4). A finer meshing was placed around the central electrode for high resolution view of absolute electric field $(10 \mathrm{~nm} \times 10 \mathrm{~nm})$. The resulting horizontal electric field was plotted as a function of length at two vertical points in the dielectric layer. 


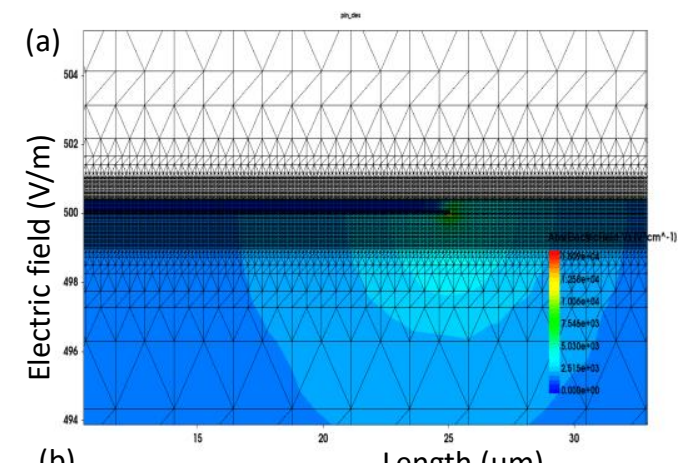

(c)
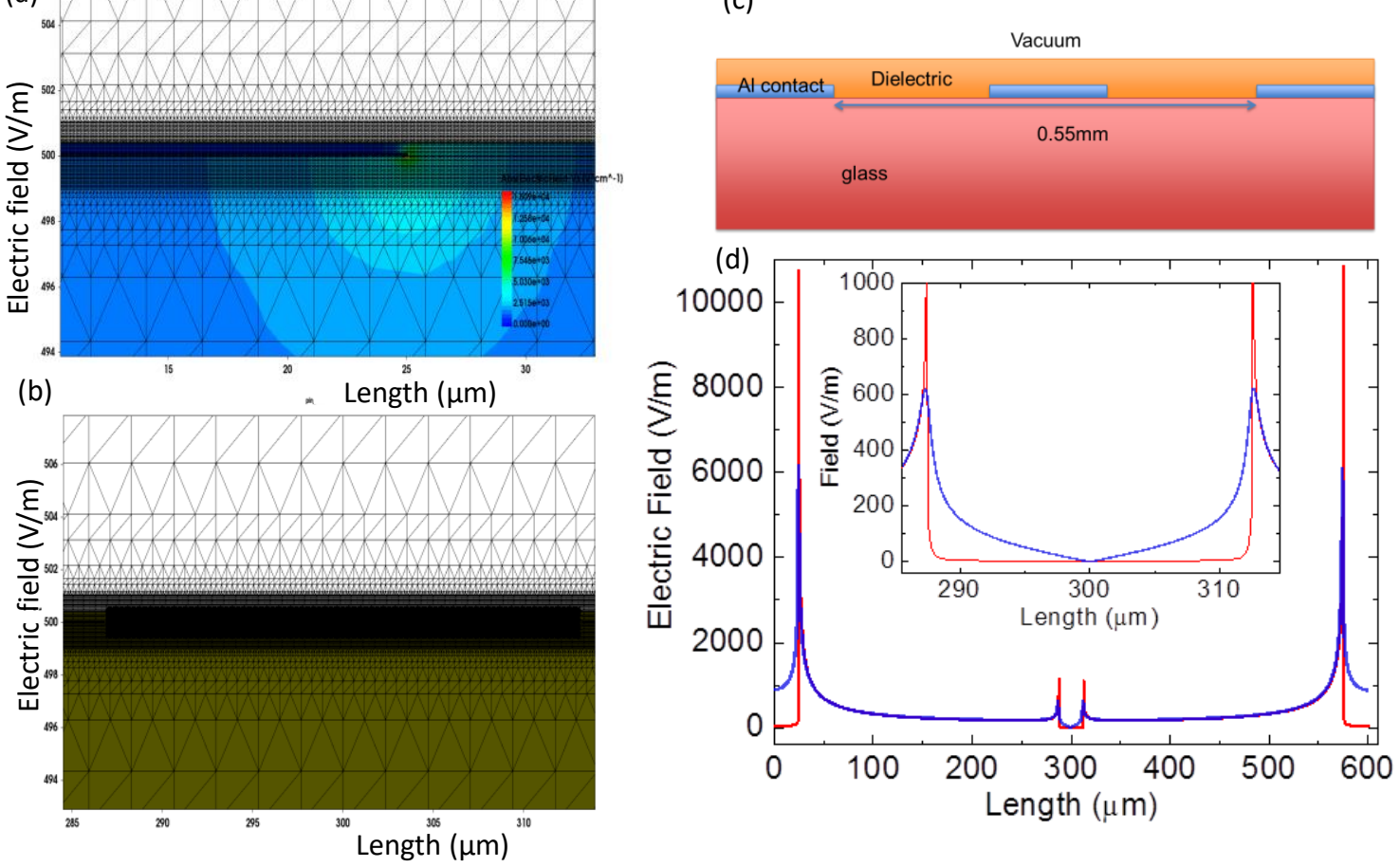

Figure 6.17 Meshing scheme for the simulated device stack and field distribution in insulating slab. (a) A refinement of $100 \mathrm{~nm}$ x $100 \mathrm{~nm}$ was made surrounding the dielectric and source/drain $\mathrm{Al}$ contacts. (b)The central Al electrode had meshing of $10 \mathrm{~nm}$ x $10 \mathrm{~nm}$. The global mesh (glass substrate below, and air above) was set to $10 \mu \mathrm{m}$ by $10 \mu \mathrm{m}$. (c) The schematics of the device setup for the simulations. (d)The plot shows the simulated electric field distribution within the dielectric layer. The blue curve shows the field distribution as seen within the dielectric slab. The inset shows the region of interest, namely, the field distribution in the dielectric layer just above the middle (gate) electrode.

Although the device dimensions were scaled down in the simulation, the magnitude of the field was changed to mimic experimental conditions. Figure 6.17 (d) shows the electric field distribution between the two electrodes, and the inset shows the region of interest on top of the gate electrode. As expected, the magnitude of the field is the maximum at the side electrodes with a small penetration in the dielectric layer. A further redistribution arises due to the central electrode where the field is zero at the center as 
shown in the inset of figure 6.17 (d). Viewing from the top of the dielectric layer the field penetration within the dielectric layer is seen to be roughly $1 / 3$ the value near the edge, which falls off closer to the center of the electrode [inset of figure 6.17 (d)]. Since the channel length of the FETs were $>50 \mu \mathrm{m}$, it is expected that there will be some influence of the field roughly at $1 / 3$ of the dielectric layer from the bottom while lateral poling. Additionally, the simulations only considered a slab of a dielectric with the same $\kappa$ as PVDF-TrFE but not a real ferroelectric with spontaneous polarization. The simulations highlight that if the FETs are away from the center of the gate electrode, the field penetration is high. This was observed for the FET shown in figure 6.15 (a), where most likely the entire dielectric layer is laterally polarized with the applied field strength, destroying the vertical polarization. These observations show that the optimum condition of textured poling for enhancing the FET characteristics is when the top of the dielectric (at the semiconductor-insulator interface) is still vertically polarized but only the bottom 1/3-1/4 portion of the dielectric is laterally poled. We further note that the applied electric fields quoted here are based on the applied voltages to the lateral electrodes; the simulations show that the actual field felt at the central electrode would be only around $2 \%$ of the applied field or even lower.

In summary, the strategy of textured poling of the polymer ferroelectric layer dramatically reduces the gate leakage current and enhances transport in organic FETs. By applying a lateral electric field near the gate electrode to an already vertically poled PVDFTrFE layer, the on/off ratios are found to be upwards of $10^{5}$ in TIPS-pentacene FETs. Carrier mobilities measured under ambient conditions are an order of magnitude higher for textured poled TIPS-pentacene FETs compared to vertically poled FETs. Without any 
specific processing of the TIPS-pentacene films and using the strategy of only textured poling the ferroelectric dielectric, ambient condition $\mu_{\mathrm{FET}}$ (holes) easily reaches $1 \mathrm{~cm}^{2} / \mathrm{Vs}$. 


\section{Summary and suggested future works}

\subsection{Summary}

In this thesis we showed that the ferroelectric dielectric PVDF-TrFE, where the polarization strength can be tuned with temperature, provides a mechanism for understanding charge transport in the rapidly growing field of small molecule and polymerbased thin film transistors. The charge transport in ferroelectric-based organic FETs is strongly influenced by a polarization dominant transport rather than an energetic disorder within the framework of a hopping transport. When the gate insulator is sufficiently polar, Fröhlich polarons play an important role in interfacial transport. This effect is more pronounced for thinner dielectric films. By fabricating stacked $\mathrm{PVDF}-\mathrm{TrFE} / \mathrm{SiO}_{2}$ dielectrics, the surface between pentacene and PVDF-TrFE remains unchanged but since the gate electrode is in contact with the $\mathrm{SiO}_{2}$ layer, the polarization field is absent. By comparing the stacked devices with single layer PVDF-TrFE pentacene OFETs, temperature-dependent mobility due to the polarization fluctuation due to the dielectric layer was extracted.

Drop-casted TIPS-pentacene films exhibit single-trap-level SCLC, similar to highpurity crystalline organic molecules. The origin of these traps could be structural or other chemical defects, which typically give rise to discrete trap states and may occur at the grain boundaries. A bulk-transport measurement from TIPS-pentacene clearly shows a negative coefficient of mobility beyond $180 \mathrm{~K}$. A similar behavior is seen in FET transport when a ferroelectric dielectric, PVDF-TrFE, is used. As such, the FET mobilities are lower than the minimum condition mobility required for observing disorder-free transport, yet the 
presence of a polarization field of the ferroelectric dielectric results in a transport mechanism, mimicking band-like transport beyond $200 \mathrm{~K}$. This is attributed to an ease of a de-trapping mechanism owing to the nature of discrete traps. This paves the way for exploring band-like transport $(\mathrm{d} \mu / \mathrm{dT}<0)$ in other solution-processable $\pi$-conjugated molecules and polymers with discrete trap levels by utilizing ferroelectric dielectrics in FET geometries.

We demonstrated that the dipole engineering of the ferroelectric dielectric PVDFTrFE enhances FET properties in particular the subthreshold swing can change by an order of magnitude, and the carrier mobility is enhanced by two order of magnitude. Polarization rotation in PVDF-TrFE has a profound effect on the transport properties of organic FETs. The subthreshold swing decreases, and the overall FET properties are enhanced when the ferroelectric layer is vertically poled with an external field. The direction of polarization for the V-poled PVDF-TrFE film is chosen such that it is in the same direction as the gate electric field, boosting the screening charge accumulation in the channel region. Irrespective of the organic semiconductor layer in the FET, results show that polarization orientation in the polymer ferroelectric layer directly controls FET performance. Orienting and reorienting the polarization direction serves as a tuning parameter for manipulating SS and carrier mobilities in organic FETs. L-poling of a V-poled PVDF-TrFE film shows changes in carrier transport in FETs as well as reduces the overall capacitance in MIM structures. This study highlights new design principles for organic FETs in lowering SS and enhancing transport properties by orienting the polarization direction in the ferroelectric dielectric layer. It has also been demonstrated that the strategy of textured poling of the polymer ferroelectric layer dramatically reduces the gate leakage current and 
enhances transport in organic FETs. The texturing achieved by first vertically poling the PVDF-TrFE film and then laterally poling close to the gate electrode results in improved mobility, on/off ratio, and subthreshold swing. TIPS-pentacene FETs, for example, show an improvement of more than an order of magnitude in carrier mobility and on/off ratio under ambient condition and operating voltages below $-4 \mathrm{~V}$. This study opens the prospect of achieving low-operating, high-performance, low-cost organic FETs by a textured polarization of the ferroelectric dielectric without any expensive patterning techniques.

\subsection{Suggested future works}

\subsubsection{Fast switching organic FETs by dipole engineering of PVDF-TrFE}

In chapter 6 we saw that dipole engineering of PVDF-TrFE with external field results in improved FET properties. We wish to expand the study on dipole engineering of ferroelectric polymers such as PVDF-TrFE to enhance the switching time in organic FETs. Low-cost, large area, solution processed, thin film polymer transistors are emerging as a next-generation technology, allowing electronic components to be integrated into flexible substrates. However, polymer thin film transistors suffer from low switching times, rendering their use in analytical and digital logic circuits problematic. Fast switching times in polymer transistors may be achieved by miniaturizing the device size, but this requires nanolithography, which is impractical for polymers and low-cost fabrication methods like printing. It would be worthwhile to utilize low-cost strategies for materials engineering to improve switching times by orders of magnitude compared to the present state-of-the-art thin film polymer transistors, which will open up the polymer circuit technology for many new applications. An experimental method based on a comparative study on unpoled, 
vertically poled and textured poled PVDF-TrFE FETs may be fabricated and characterized to get the crucial parameters that controls switching time. The transistor switching speed and the frequency bandwidth measurement can be obtained as described in previous works. ${ }^{115}$

As a further extension of this work, the switching times in FETs could be enhanced by optimized dipole engineering of PVDF-TrFE. Dipole engineering of the dielectric layer, using low-cost solvent processing and poling polymer ferroelectric dielectrics with external field, is likely to have a transformative impact on technology where fast switching times may be realized in long-channel printable thin film organic field-effect transistors and logic circuits.

\subsubsection{Carrier transport mechanism and FET mobility using non-linear optics}

In our research, we use current-voltage measurements and analyze data to extract charge mobility in an organic FET. This method involves the contribution of contact resistance, the size and geometry of device, and the property of dielectric. Alternative methods could be developed which does not involve contact resistance, or the influence of dielectrics. Nonlinear optical methods such as Time-resolved microscopic optical second harmonic generation (TRM-SHG) and Transient electric field-induced second-harmonic generation (EFISHG) are well known to probe the carrier mobility and dynamical carrier transport in organic semiconductors. ${ }^{116-120}$

The TRM-SHG approach, based on the second order susceptibility, gives information about the charge transport motion without considering the effect of the electrode injection process. It is free from the issues of contact resistance. This technique 
allows selective and direct probing of dynamic carrier motion in organic semiconductor materials. This technique reveals the dynamic changes in SHG intensity profiles. The TRM-SHG images can be directly visualized using a charge-coupled device (CCD) camera. Carrier mobility in semiconductors is estimated by using ${ }^{76}$,

$$
\mu=\left(\frac{1}{2} \frac{x^{2}}{(V-V d r o p) \tau}\right)
$$

where $x$ is the distance from the edge of the source electrode to the front edge of the SHG emission called transported carrier distance. $\mathrm{V}$ is the external voltage applied to the source electrode and $V_{\text {drop }}$ is the potential drop. The time delay, $\tau$, is the delay between the laser pulse and the voltage pulse. The current setup has some limitations and we hope to build the imaging part of the setup using a CCD and a microscope attachment.

EFISHG, based on the third-order susceptibility, allows direct and selective probing of dynamic carrier motion in field-effect transistors. Under the application of some DC field and irradiation of a laser light on the organic materials, the nonlinear polarization, $P$, will induce an interaction between electromagnetic fields and electrons in the organic materials. This is a basic principle of EFISHG measurement. As the electron distribution of molecules is distorted by the presence of the dc field $E(0)$, this type of nonlinear polarization is induced even in a centrosymmetric molecular system and $P(2 \omega)$ can, be presented by equation,

$$
P(2 \omega)=\chi^{3}(2 \omega ; 0, \omega, \omega) E(0) E i(\omega) E j(\omega)
$$

where $\chi^{3}$ is the third-order-nonlinear susceptibility and $E(0)$ is a local electrostatic field. $E_{\mathrm{i}}$ and $E_{\mathrm{j}}$ represent the electric field of incident light. EFISHG enables the probing of the electric field distribution in the FET channel and generates the signals that follow above 
equation. Hence, one can obtain carrier distribution along the channel from EFISHG measurement.

\subsubsection{Low operating organic FETs for sensing applications}

An FET is a charge sensor. Any charge variation on the gate will induce a current variation in the channel. Low operating FETs would sense a small change in current variation in the channel. This ability of organic FETs to detect charge variations could be exploited for realizing chemo- and bio-sensors. The organic FET sensor represents an innovation as the sensing area is physically separated from the channel area and the device response does not depend on the specific characteristics of the device materials. This feature makes it possible to reuse the device as one can simply replace sensing area without destroying the FET. Several researches have shown the sensing applications of organic FETs. ${ }^{121-123}$

(a)

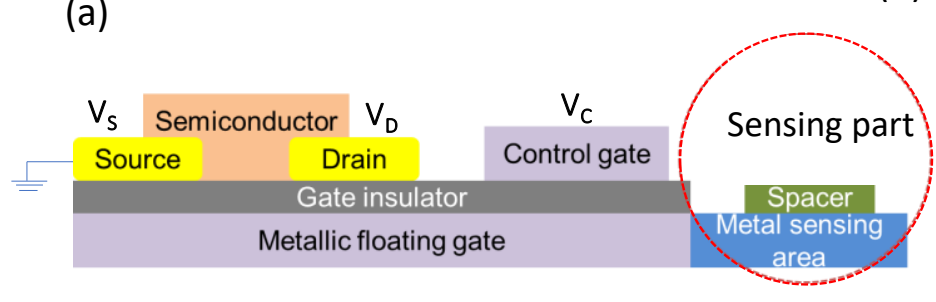

(b)

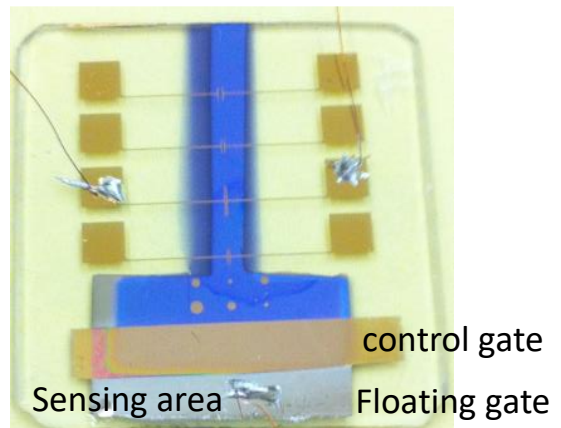

Figure 7.1 (a) FET design as a sensor and (b) a photograph of such device based on pentacene FET

Figure 7.1 (a) shows an example of FET design for a sensor and figure 7.1 (b) shows a photograph of such an FET based on pentacene. The active area of FET device is mounted for protection and sensing area is exposed to the material to be sensed and can be removed 
afterwards to be replaced by a new sensing part. Any modification of the charge will change voltage difference between the floating gate and source $\left(\Delta \mathrm{V}_{\mathrm{F}}\right)$ which is given by ${ }^{124}$,

$$
\Delta V_{T h}=\Delta V_{F}=-\frac{Q_{s e n s}}{C_{T o t}}
$$

where $\mathrm{Q}_{\text {sens }}$ is the total surface charge and $\mathrm{C}_{\text {Tot }}$ is total capacitance. PVDF-TrFE has a high dielectric constant which offers low operating FETs, meaning more sensitive detection of charge accumulation. 


\section{References}

1. Chiang, C. K.; Fincher, C. R.; Park, Y. W.; Heeger, A. J.; Shirakawa, H.; Louis, E. J.; Gau, S. C.; MacDiarmid, A. G., Electrical Conductivity in Doped Polyacetylene. Phys. Rev. Lett. 1977, 39 (17), 1098-1101.

2. Tsumura, A., applied physic letters 1986, 49, 2.

3. Khan, S. U. M., Organic Semiconductors. Salem Press: 2013.

4. Kim, J. H.; Yun, S. W.; An, B.-K.; Han, Y. D.; Yoon, S.-J.; Joo, J.; Park, S. Y., Remarkable Mobility Increase and Threshold Voltage Reduction in Organic Field-Effect Transistors by Overlaying Discontinuous Nano-Patches of Charge-Transfer Doping Layer on Top of Semiconducting Film. 2013, 25 (5), 719-724.

5. $\mathrm{Xu}, \mathrm{W} . ;$ Rhee, S.-W., Low-operating voltage organic field-effect transistors with high-k cross-linked cyanoethylated pullulan polymer gate dielectrics. Journal of Materials Chemistry 2009, 19 (29), 5250-5257.

6. Gundlach, D. J.; Lin, Y. Y.; Jackson, T. N.; Nelson, S. F.; Schlom, D. G., Pentacene organic thin-film transistors-molecular ordering and mobility. IEEE Electron Device Letters 1997, 18 (3), 87-89.

7. Dinca, L. E.; De Marchi, F.; MacLeod, J. M.; Lipton-Duffin, J.; Gatti, R.; Ma, D.; Perepichka, D. F.; Rosei, F., Pentacene on Ni(111): room-temperature molecular packing and temperature-activated conversion to graphene. Nanoscale 2015, 7 (7), 32633269. 
8. Yamamoto, T.; Takimiya, K., Facile Synthesis of Highly $\pi$-Extended Heteroarenes, Dinaphtho[2,3-b:2 ${ }^{\mathfrak{c}}, 3^{6}$-f $]$ chalcogenopheno[3,2-b]chalcogenophenes, and Their Application to Field-Effect Transistors. J. Am. Chem. Soc. 2007, 129 (8), 2224-2225.

9. Zschieschang, U.; Ante, F.; Kälblein, D.; Yamamoto, T.; Takimiya, K.; Kuwabara, H.; Ikeda, M.; Sekitani, T.; Someya, T.; Nimoth, J. B.; Klauk, H., Dinaphtho[2,3-b:2',3'-f]thieno[3,2-b]thiophene (DNTT) thin-film transistors with improved performance and stability. Organic Electronics 2011, 12 (8), 1370-1375.

10. Park, S. K.; Jackson, T. N.; Anthony, J. E.; Mourey, D. A., High mobility solution processed 6,13-bis(triisopropyl-silylethynyl) pentacene organic thin film transistors. Applied Physics Letters 2007, 91 (6), 063514.

11. Park, J. G.; Vasic, R.; Brooks, J. S.; Anthony, J. E., Characterization of functionalized pentacene field-effect transistors and its logic gate application. J. Appl. Phys. 2006, 100 (4), 044511.

12. Sakanoue, T.; Sirringhaus, H., Band-like temperature dependence of mobility in a solution-processed organic semiconductor. Nat Mater 2010, 9 (9), 736-740.

13. Davi, N. F. M. a. E. A., Electronic Processes in Non-crystalline Materials. second ed.; 1979.

14. Klauk, H., Organic thin-film transistors. Chemical Society Reviews 2010, 39 (7), 2643-2666.

15. Zaumseil, J.; Sirringhaus, H., Electron and Ambipolar Transport in Organic FieldEffect Transistors. Chem. Rev. 2007, 107 (4), 1296-1323.

16. Vissenberg, M. C. J. M.; Matters, M., Theory of the field-effect mobility in amorphous organic transistors. Physical Review B 1998, 57 (20), 12964-12967. 
17. Koh, S. E.; Delley, B.; Medvedeva, J. E.; Facchetti, A.; Freeman, A. J.; Marks, T. J.; Ratner, M. A., Quantum Chemical Analysis of Electronic Structure and n- and pType Charge Transport in Perfluoroarene-Modified Oligothiophene Semiconductors. The Journal of Physical Chemistry B 2006, 110 (48), 24361-24370.

18. Chesterfield, R. J.; McKeen, J. C.; Newman, C. R.; Frisbie, C. D.; Ewbank, P. C.; Mann, K. R.; Miller, L. L., Variable temperature film and contact resistance measurements on operating n-channel organic thin film transistors. J. Appl. Phys. 2004, 95 (11), 6396-6405.

19. Waragai, K.; Akimichi, H.; Hotta, S.; Kano, H.; Sakaki, H., Charge transport in thin films of semiconducting oligothiophenes. Physical Review B 1995, 52 (3), 1786-1792. 20. Horowitz, G., Organic thin film transistors: From theory to real devices. J. Mater. Res. 2004, 19 (7), 1946-1962.

21. Jurchescu, O. D.; Baas, J.; Palstra, T. T. M., Effect of impurities on the mobility of single crystal pentacene. 2004, 84 (16), 3061-3063.

22. Kittel, C., Introduction to Solid State Physics. Book 2004, 8th editioh, 704.

23. Lovinger, A. J., Ferroelectric Polymers. Science 1983, 220 (4602), 1115-1121.

24. Oliveira, F.; Leterrier, Y.; Månson, J.-A.; Sereda, O.; Neels, A.; Dommann, A.; Damjanovic, D., Process influences on the structure, piezoelectric, and gas-barrier properties of PVDF-TrFE copolymer. Journal of Polymer Science Part B: Polymer Physics 2014, 52 (7), 496-506.

25. Furukawa, T., Piezoelectricity and pyroelectricity in polymers. IEEE Transactions on Electrical Insulation 1989, 24 (3), 375-394. 
26. Li, M.; Wondergem, H. J.; Spijkman, M.-J.; Asadi, K.; Katsouras, I.; Blom, P. W. M.; de Leeuw, D. M., Revisiting the $\delta$-phase of poly(vinylidene fluoride) for solutionprocessed ferroelectric thin films. Nature Materials 2013, 12, 433.

27. G., A. I.; F., M. N., 1969, 18, 41.

28. Laudari, A.; Guha, S., Polarization-induced transport in ferroelectric organic fieldeffect transistors. Journal of Applied Physics 2015, 117 (10), 105501.

29. Nan, C.-W.; Weng, G. J., Influence of polarization orientation on the effective properties of piezoelectric composites. J. Appl. Phys. 2000, 88 (1), 416-423.

30. Khanra, S.; Ghosh, K.; Ferreira, F. F.; Alves, W. A.; Punzo, F.; Yu, P.; Guha, S., Probing nonlinear optical coefficients in self-assembled peptide nanotubes. Physical Chemistry Chemical Physics 2017, 19 (4), 3084-3093.

31. Berge, B.; Wicker, A.; Lajzerowicz, J.; Legrand, J. F., Second-Harmonic Generation of Light and Evidence of Phase Matching in Thin Films of P(VDF-TrFE) Copolymers. EPL (Europhysics Letters) 1989, 9 (7), 657.

32. Dong-Jin, P.; Jaehyun, M.; Byung-Ok, P., An Analysis on the Electrical Short in a Large Area Organic Thin Film Transistor Array with the Poly(4-vinylphenol) Gate Dielectrics. Jpn. J. Appl. Phys. 2009, 48 (8R), 081504.

33. P. Parisse, S. P., M. Passacantando, L. Ottaviano, Thin Sol. Films 2007, 515, 8316. 34. Simon M. Sze, K. K. N., Physics of Semiconductor Devices. 2006, 832.

35. Qi, Y.; Rappe, A. M., Designing Ferroelectric Field-Effect Transistors Based on the Polarization-Rotation Effect for Low Operating Voltage and Fast Switching. Physical Review Applied 2015, 4 (4), 044014. 
36. Zschieschang, U.; Bader, V. P.; Klauk, H., Below-one-volt organic thin-film transistors with large on/off current ratios. Organic Electronics 2017, 49, 179-186.

37. Zschieschang, U.; Klauk, H., Low-voltage organic transistors with steep subthreshold slope fabricated on commercially available paper. Organic Electronics 2015, $25,340-344$.

38. Blülle, B.; Häusermann, R.; Batlogg, B., Approaching the Trap-Free Limit in Organic Single-Crystal Field-Effect Transistors. Physical Review Applied 2014, 1 (3), 034006.

39. Ben Streetman, S. B., Solid State Electronic Devices book 2006.

40. Adil, D. Light scattering studies of organic field effect transistors. University of Missouri, 2013.

41. CRC Handbook of Chemistry and Physics, 84th Edition Edited by David R. Lide (National Institute of Standards and Technology). CRC Press LLC: Boca Raton. 2003. 2616 pp. \$139.95. ISBN 0-8493-0484-9. J. Am. Chem. Soc. 2004, 126 (5), 1586-1586.

42. Coropceanu, V.; Cornil, J.; da Silva Filho, D. A.; Olivier, Y.; Silbey, R.; Brédas, J.-L., Charge Transport in Organic Semiconductors. Chem. Rev. 2007, 107 (4), 926-952.

43. Houili, H.; Picon, J. D.; Zuppiroli, L.; Bussac, M. N., Polarization effects in the channel of an organic field-effect transistor. J. Appl. Phys. 2006, 100 (2), 023702.

44. Konezny, S. J.; Bussac, M. N.; Zuppiroli, L., Hopping and trapping mechanisms in organic field-effect transistors. Physical Review B 2010, 81 (4), 045313.

45. Naber, R. C. G.; Blom, P. W. M.; Marsman, A. W.; de Leeuw, D. M., Low voltage switching of a spin cast ferroelectric polymer. Appl. Phys. Lett. 2004, 85 (11), 2032-2034. 
46. Naber, R. C. G.; Tanase, C.; Blom, P. W. M.; Gelinck, G. H.; Marsman, A. W.; Touwslager, F. J.; Setayesh, S.; de Leeuw, D. M., High-performance solution-processed polymer ferroelectric field-effect transistors. Nat Mater 2005, 4 (3), 243-248.

47. Fujisaki, S.; Ishiwara, H.; Fujisaki, Y., Low-voltage operation of ferroelectric poly(vinylidene fluoride-trifluoroethylene) copolymer capacitors and metal-ferroelectricinsulator-semiconductor diodes. Applied Physics Letters 2007, 90 (16), 162902.

48. Nguyen, C. A.; Mhaisalkar, S. G.; Ma, J.; Lee, P. S., Enhanced organic ferroelectric field effect transistor characteristics with strained poly(vinylidene fluoridetrifluoroethylene) dielectric. Organic Electronics 2008, 9 (6), 1087-1092.

49. Zheng, Y.; Ni, G.-X.; Toh, C.-T.; Tan, C.-Y.; Yao, K.; Özyilmaz, B., Graphene Field-Effect Transistors with Ferroelectric Gating. Phys. Rev. Lett. 2010, 105 (16), 166602. 50. Bässler, H., Charge Transport in Disordered Organic Photoconductors a Monte Carlo Simulation Study. physica status solidi (b) 1993, 175 (1), 15-56.

51. Hulea, I. N.; Fratini, S.; Xie, H.; Mulder, C. L.; Iossad, N. N.; Rastelli, G.; Ciuchi, S.; Morpurgo, A. F., Tunable Frohlich polarons in organic single-crystal transistors. Nat Mater 2006, 5 (12), 982-986.

52. Xia, Y.; Cho, J. H.; Lee, J.; Ruden, P. P.; Frisbie, C. D., Comparison of the Mobility-Carrier Density Relation in Polymer and Single-Crystal Organic Transistors Employing Vacuum and Liquid Gate Dielectrics. Advanced Materials 2009, 21 (21), 21742179.

53. Senanayak, S. P.; Guha, S.; Narayan, K. S., Polarization fluctuation dominated electrical transport processes of polymer-based ferroelectric field effect transistors. Physical Review B 2012, 85 (11), 115311. 
54. Knipp, D.; Street, R. A.; Völkel, A.; Ho, J., Pentacene thin film transistors on inorganic dielectrics: Morphology, structural properties, and electronic transport. J. Appl. Phys. 2003, 93 (1), 347-355.

55. Guo, D.; Miyadera, T.; Ikeda, S.; Shimada, T.; Saiki, K., Analysis of charge transport in a polycrystalline pentacene thin film transistor by temperature and gate bias dependent mobility and conductance. Journal of Applied Physics 2007, 102 (2), 023706.

56. Dunn, L.; Dodabalapur, A., Temperature dependent transient velocity and mobility studies in an organic field effect transistor. J. Appl. Phys. 2010, 107 (11), 113714.

57. Gundlach, D. J.; Royer, J. E.; Park, S. K.; Subramanian, S.; Jurchescu, O. D.; Hamadani, B. H.; Moad, A. J.; Kline, R. J.; Teague, L. C.; Kirillov, O.; Richter, C. A.; Kushmerick, J. G.; Richter, L. J.; Parkin, S. R.; Jackson, T. N.; Anthony, J. E., Contactinduced crystallinity for high-performance soluble acene-based transistors and circuits. Nature Materials 2008, 7, 216.

58. Monroe, D., Hopping in Exponential Band Tails. Phys. Rev. Lett. 1985, 54 (2), 146149.

59. Ukah, N. B.; Granstrom, J.; Sanganna Gari, R. R.; King, G. M.; Guha, S., Lowoperating voltage and stable organic field-effect transistors with poly (methyl methacrylate) gate dielectric solution deposited from a high dipole moment solvent. Applied Physics Letters 2011, 99 (24), 243302.

60. Knotts, G.; Bhaumik, A.; Ghosh, K.; Guha, S., Enhanced performance of ferroelectric-based all organic capacitors and transistors through choice of solvent. Appl. Phys. Lett. 2014, 104 (23), 233301.

61. W., X.; S.-W., R., 2009, 19, 5250. 
62. Tashiro, K.; Itoh, Y.; Kobayashi, M.; Tadokoro, H., Polarized Raman spectra and LO-TO splitting of poly(vinylidene fluoride) crystal form I. Macromolecules 1985, 18 (12), 2600-2606.

63. Nakhmanson, S. M.; Korlacki, R.; Johnston, J. T.; Ducharme, S.; Ge, Z.; Takacs, J. M., Vibrational properties of ferroelectric \$lensuremath\{ \beta\}\$-vinylidene fluoride polymers and oligomers. Physical Review B 2010, 81 (17), 174120.

64. Tashiro, K.; Kobayashi, M., Vibrational spectroscopic study of the ferroelectric phase transition in vinylidene fluoride-trifluoroethylene copolymers: 1. Temperature dependence of the Raman spectra. Polymer 1988, 29 (3), 426-436.

65. Adil, D.; Guha, S., Surface-enhanced Raman spectroscopic studies of the Aupentacene interface: A combined experimental and theoretical investigation. The Journal of Chemical Physics 2013, 139 (4), 044715.

66. Mott, N. F.; Davis, E. A., Electronic processes in non-crystalline materials. Clarendon Press ; Oxford University Press: Oxford; New York, 1979.

67. Miller, A.; Abrahams, E., Impurity Conduction at Low Concentrations. Phys. Rev. 1960, $120(3), 745-755$.

68. Borsenberger, P. M.; Pautmeier, L.; Bässler, H., Charge transport in disordered molecular solids. The Journal of Chemical Physics 1991, 94 (8), 5447-5454.

69. Pope, M.; Swenberg, C. E., Electronic Processes in Organic Crystals and Polymer. Second ed.; Oxford University Press: Oxford, 1999.

70. Arif, M.; Yun, M.; Gangopadhyay, S.; Ghosh, K.; Fadiga, L.; Galbrecht, F.; Scherf, U.; Guha, S., Polyfluorene as a model system for space-charge-limited conduction. Physical Review B 2007, 75 (19), 195202. 
71. Chua, L.-L.; Zaumseil, J.; Chang, J.-F.; Ou, E. C. W.; Ho, P. K. H.; Sirringhaus, H.; Friend, R. H., General observation of n-type field-effect behaviour in organic semiconductors. Nature 2005, 434 (7030), 194-199.

72. Podzorov, V.; Menard, E.; Rogers, J. A.; Gershenson, M. E., Hall Effect in the Accumulation Layers on the Surface of Organic Semiconductors. Phys. Rev. Lett. 2005, 95 (22), 226601.

73. Jun, T.; Kazuhito, T.; Yoshinobu, A.; Taishi, T.; Yoshihiro, I., Hall Effect of Quasi-Hole Gas in Organic Single-Crystal Transistors. Japanese Journal of Applied Physics 2005, 44 (11L), L1393.

74. Meneau, A. Y. B.; Olivier, Y.; Backlund, T.; James, M.; Breiby, D. W.; Andreasen, J. W.; Sirringhaus, H., Temperature Dependence of Charge Localization in High-Mobility, Solution-Crystallized Small Molecule Semiconductors Studied by Charge Modulation Spectroscopy. Advanced Functional Materials 2015, doi: 10.1002/adfm.201502502.

75. Fratini, S.; Ciuchi, S., Bandlike Motion and Mobility Saturation in Organic Molecular Semiconductors. Phys. Rev. Lett. 2009, 103 (26), 266601.

76. Kohei, M.; Takaaki, M.; Mitsumasa, I., Band-like transport observed in TIPSpentacene thin film by time-resolved microscopic optical second-harmonic generation imaging. Applied Physics Express 2015, 8 (4), 041601.

77. Stafstrom, S., Electron localization and the transition from adiabatic to nonadiabatic charge transport in organic conductors. Chemical Society Reviews 2010, 39 (7), 2484-2499. 
78. Chen, Y.; Yi, H. T.; Podzorov, V., High-Resolution ac Measurements of the Hall Effect in Organic Field-Effect Transistors. Physical Review Applied 2016, 5 (3), 034008.

79. Yi, H. T.; Gartstein, Y. N.; Podzorov, V., Charge carrier coherence and Hall effect in organic semiconductors. Scientific Reports 2016, 6, 23650.

80. Schein, L. B.; Duke, C. B.; McGhie, A. R., Observation of the Band-Hopping Transition for Electrons in Naphthalene. Phys. Rev. Lett. 1978, 40 (3), 197-200.

81. Wang, L. J.; Peng, Q.; Li, Q. K.; Shuai, Z., Roles of inter- and intramolecular vibrations and band-hopping crossover in the charge transport in naphthalene crystal. The Journal of Chemical Physics 2007, 127 (4), 044506.

82. Wang, L.; Prezhdo, O. V.; Beljonne, D., Mixed quantum-classical dynamics for charge transport in organics. Physical Chemistry Chemical Physics 2015, 17 (19), 1239512406.

83. Senanayak, S. P.; Narayan, K. S., Strategies for Fast-Switching in All-Polymer Field Effect Transistors. Advanced Functional Materials 2014, 24 (22), 3324-3331.

84. Naber, R. C. G.; Mulder, M.; de Boer, B.; Blom, P. W. M.; de Leeuw, D. M., High charge density and mobility in poly(3-hexylthiophene) using a polarizable gate dielectric. Organic Electronics 2006, 7 (3), 132-136.

85. Brondijk, J. J.; Asadi, K.; Blom, P. W. M.; de Leeuw, D. M., Physics of organic ferroelectric field-effect transistors. Journal of Polymer Science Part B: Polymer Physics 2012, $50(1), 47-54$.

86. Zirkl, M.; Sawatdee, A.; Helbig, U.; Krause, M.; Scheipl, G.; Kraker, E.; Ersman, P. A.; Nilsson, D.; Platt, D.; Bodö, P.; Bauer, S.; Domann, G.; Stadlober, B., An All-Printed Ferroelectric Active Matrix Sensor Network Based on Only Five Functional 
Materials Forming a Touchless Control Interface. Advanced Materials 2011, 23 (18), 20692074.

87. Lee, J.-H.; Yoon, H.-J.; Kim, T. Y.; Gupta, M. K.; Lee, J. H.; Seung, W.; Ryu, H.; Kim, S.-W., Micropatterned P(VDF-TrFE) Film-Based Piezoelectric Nanogenerators for Highly Sensitive Self-Powered Pressure Sensors. Advanced Functional Materials 2015, 25 (21), 3203-3209.

88. Li, Y. C.; Tjong, S. C.; Li, R. K. Y., Electrical conductivity and dielectric response of poly(vinylidene fluoride)-graphite nanoplatelet composites. Synthetic Metals 2010, 160 (17), 1912-1919.

89. Kimura, H.; Hanyu, T.; Kameyama, M.; Fujimori, Y.; Nakamura, T.; Takasu, H., Complementary ferroelectric-capacitor logic for low-power logic-in-memory VLSI. IEEE Journal of Solid-State Circuits 2004, 39 (6), 919-926.

90. Sakai, S.; Takahashi, M., Recent Advances in Ferroelectric-Gate Field-EffectTransistor Technology. Integrated Ferroelectrics 2011, 124 (1), 140-146.

91. Mathews, S.; Ramesh, R.; Venkatesan, T.; Benedetto, J., Ferroelectric Field Effect Transistor Based on Epitaxial Perovskite Heterostructures. Science 1997, 276 (5310), 238240.

92. Fu, H.; Cohen, R. E., Polarization rotation mechanism for ultrahigh electromechanical response in single-crystal piezoelectrics. Nature 2000, 403 (6767), 281283.

93. Nagarajan, V.; Roytburd, A.; Stanishevsky, A.; Prasertchoung, S.; Zhao, T.; Chen, L.; Melngailis, J.; Auciello, O.; Ramesh, R., Dynamics of ferroelastic domains in ferroelectric thin films. Nat Mater 2003, 2 (1), 43-47. 
94. Hlinka, J.; Márton, P., Phenomenological model of a $90^{\circ}$ domain wall in $\mathrm{BaTiO}_{3}-$ type ferroelectrics. Physical Review B 2006, 74 (10), 104104.

95. Bourgaux-Leonard, C.; Legrand, J. F.; Renault, A.; Delzenne, P., Annealing effects in ferroelectric poly(vinylidene fluoride-trifluoroethylene) copolymers: real-time studies using synchrotron radiation. Polymer 1991, 32 (4), 597-604.

96. Choi, H. H.; Cho, K.; Frisbie, C. D.; Sirringhaus, H.; Podzorov, V., Critical assessment of charge mobility extraction in FETs. Nature Materials 2017, 17, 2.

97. Podzorov, V.; Sysoev, S. E.; Loginova, E.; Pudalov, V. M.; Gershenson, M. E., Single-crystal organic field effect transistors with the hole mobility $\sim 8 \mathrm{~cm} 2 / \mathrm{Vs}$. Applied Physics Letters 2003, 83 (17), 3504-3506.

98. Grünewald, M.; Thomas, P.; Würtz, D., A Simple Scheme for Evaluating Field Effect Data. physica status solidi (b) 1980, 100 (2), K139-K143.

99. Kalb, W. L.; Batlogg, B., Calculating the trap density of states in organic fieldeffect transistors from experiment: A comparison of different methods. Physical Review B 2010, 81 (3), 035327.

100. Diemer, P. J.; Lamport, Z. A.; Mei, Y.; Ward, J. W.; Goetz, K. P.; Li, W.; Payne, M. M.; Guthold, M.; Anthony, J. E.; Jurchescu, O. D., Quantitative analysis of the density of trap states at the semiconductor-dielectric interface in organic field-effect transistors. Applied Physics Letters 2015, 107 (10), 103303.

101. Laudari, A.; Mazza, A. R.; Daykin, A.; Khanra, S.; Ghosh, K.; Cummings, F.; Muller, T.; Miceli, P. F.; Guha, S., Polarization Modulation in Ferroelectric Organic FieldEffect Transistors. Physical Review Applied 2018, 10 (1), 014011. 
102. Otsuka, T.; Taguchi, D.; Manaka, T.; Iwamoto, M., Direct visualization of polarization reversal of organic ferroelectric memory transistor by using charge modulated reflectance imaging. Journal of Applied Physics 2017, 122 (18), 185501.

103. Jung, S.-W.; Baeg, K.-J.; Yoon, S.-M.; You, I.-K.; Lee, J.-K.; Kim, Y.-S.; Noh, Y.-Y., Low-voltage-operated top-gate polymer thin-film transistors with high capacitance poly(vinylidene fluoride-trifluoroethylene)/poly(methyl methacrylate) dielectrics. J. Appl. Phys. 2010, 108 (10), 102810.

104. Sun, H.; Wang, Q.; Li, Y.; Lin, Y.-F.; Wang, Y.; Yin, Y.; Xu, Y.; Liu, C.; Tsukagoshi, K.; Pan, L.; Wang, X.; Hu, Z.; Shi, Y., Boost Up Carrier Mobility for Ferroelectric Organic Transistor Memory via Buffering Interfacial Polarization Fluctuation. Scientific Reports 2014, 4, 7227.

105. Guo, D.; Setter, N., Impact of Confinement-Induced Cooperative Molecular Orientation Change on the Ferroelectric Size Effect in Ultrathin P(VDF-TrFE) Films. Macromolecules 2013, 46 (5), 1883-1889.

106. Garcia-Gutierrez, M.-C.; Linares, A.; Martin-Fabiani, I.; Hernandez, J. J.; Soccio, M.; Rueda, D. R.; Ezquerra, T. A.; Reynolds, M., Understanding crystallization features of $\mathrm{P}(\mathrm{VDF}-\mathrm{TrFE})$ copolymers under confinement to optimize ferroelectricity in nanostructures. Nanoscale 2013, 5 (13), 6006-6012.

107. Martins, P.; Lopes, A. C.; Lanceros-Mendez, S., Electroactive phases of poly(vinylidene fluoride): Determination, processing and applications. Progress in Polymer Science 2014, 39 (4), 683-706. 
108. Dvey-Aharon, H.; Sluckin, T. J.; Taylor, P. L.; Hopfinger, A. J., Kink propagation as a model for poling in poly(vinylidene fluoride). Physical Review B 1980, 21 (8), 37003707.

109. Kepler, R. G.; Anderson, R. A., Ferroelectric polymers. Adv. Phys. 1992, 41 (1), 157.

110. Samara, G. A., Ferroelectricity revisited-Advances in materials and physics. In Solid State Phys., Ehrenreich, H.; Spaepen, F., Eds. Academic Press: 2001; Vol. 56, pp $239-458$.

111. Kepler, R. G.; Anderson, R. A., Ferroelectricity in polyvinylidene fluoride. J. Appl. Phys. 1978, 49 (3), 1232-1235.

112. Laudari, A.; Pickett, A.; Shahedipour-Sandvik, F.; Hogan, K.; Anthony, J. E.; He, X.; Guha, S., Textured Poling of the Ferroelectric Dielectric Layer for Improved Organic Field-Effect Transistors. Advanced Materials Interfaces 2019, 0 (0), 1801787.

113. Gundlach, D. J.; Zhou, L.; Nichols, J. A.; Jackson, T. N.; Necliudov, P. V.; Shur, M. S., An experimental study of contact effects in organic thin film transistors. J. Appl. Phys. 2006, 100 (2), 024509.

114. Lamport, Z. A.; Haneef, H. F.; Anand, S.; Waldrip, M.; Jurchescu, O. D., Tutorial: Organic field-effect transistors: Materials, structure and operation. J. Appl. Phys. 2018, 124 (7), 071101.

115. Ukah, N. B.; Senanayak, S. P.; Adil, D.; Knotts, G.; Granstrom, J.; Narayan, K. S.; Guha, S., Enhanced mobility and environmental stability in all organic field-effect transistors: The role of high dipole moment solvent. Journal of Polymer Science Part B: Polymer Physics 2013, 51 (21), 1533-1542. 
116. Manaka, T.; Lim, E.; Tamura, R.; Yamada, D.; Iwamoto, M., Probing of the electric field distribution in organic field effect transistor channel by microscopic secondharmonic generation. Applied Physics Letters 2006, 89 (7), 072113.

117. Manaka, T.; Nakao, M.; Yamada, D.; Lim, E.; Iwamoto, M., Optical second harmonic generation imaging for visualizing in-plane electric field distribution. Opt. Express 2007, 15 (24), 15964-15971.

118. Yamada, D.; Manaka, T.; Lim, E.; Tamura, R.; Weis, M.; Iwamoto, M., Injected carrier distribution in a pentacene field effect transistor probed using optical second harmonic generation. Journal of Applied Physics 2008, 104 (7), 074502.

119. Zubair, A.; Shahino Mah, A.; Dai, T.; Khaulah, S.; Takaaki, M.; Mitsumasa, I., Investigation of carrier transit motion in PCDTBT by optical SHG technique. Laser Physics 2014, 24 (10), 105701.

120. Katsuno, T.; Manaka, T.; Soejima, N.; Ishikawa, T.; Iwamoto, M., Degradation analysis of $\mathrm{AlGaN} / \mathrm{GaN}$ high electron mobility transistor by electroluminescence, electric field-induced optical second-harmonic generation, and photoluminescence imaging. Applied Physics Letters 2018, 113 (1), 012106.

121. Goetz, S. M.; Erlen, C. M.; Grothe, H.; Wolf, B.; Lugli, P.; Scarpa, G., Organic field-effect transistors for biosensing applications. Organic Electronics 2009, 10 (4), 573580.

122. Caroline, K.; Patrik, A.; Hadayat Ullah, K.; Ciril, R.-R.; Josef, B.; Sabine, S.; Jang-Joo, K.; Zhenan, B.; Christoph, K.; Paolo, P.; Wolfgang, K., Electronic biosensing with flexible organic transistor devices. Flexible and Printed Electronics 2018, 3 (3), 034003. 
123. Berto, M.; Vecchi, E.; Baiamonte, L.; Condò, C.; Sensi, M.; Di Lauro, M.; Sola, M.; De Stradis, A.; Biscarini, F.; Minafra, A.; Bortolotti, C. A., Label free detection of plant viruses with organic transistor biosensors. Sensors and Actuators B: Chemical 2019, 281, 150-156.

124. Demelas, M.; Lai, S.; Spanu, A.; Martinoia, S.; Cosseddu, P.; Barbaro, M.; Bonfiglio, A., Charge sensing by organic charge-modulated field effect transistors: application to the detection of bio-related effects. Journal of Materials Chemistry B 2013, $1(31), 3811-3819$. 


\section{Publications}

1. A. Laudari, A. Pickett, F. Shahedipour-Sandvik, K. Hogan, J. E. Anthony, X. He, and S. Guha "Textured Poling of the Ferroelectric Dielectric Layer for Improved Organic Field-Effect Transistors” Advanced Materials Interfaces, 1801787 (2019)

2. A. Laudari and S. Guha, "Temperature dependent carrier mobility in organic field-effect transistors: role of dielectrics” J. Appl. Phys. 125, 035501 (2019)

3. A. Laudari, A.R. Mazza, A. Daykin, S., K. Ghosh, F. Cummings, T. Muller, P. F. Miceli, and S. Guha "Polarization Modulation in Ferroelectric Organic Field-Effect Transistors” Phys. Rev. Applied 10, 014011 (2018)

4. A. Pickett, M. Torkkeli, T. Mukhopadhyay, B. Puttaraju, A. Laudari, A. Lauritzen, O. Bikondoa, J. K. Hansen, M. Knaapila, S. Patil, and S. Guha "Correlating charge transport to structure in deconstructed diketopyrrolopyrrole oligomers: A case study of a monomer in field-effect transistors” ACS Appl. Mater. Interfaces 10 (23), 19844-19852 (2018)

5. S.Goswami, J. L. Hernandez, M. K. Gish, J. Wang, B. Kim, A. Laudari, S. Guha, J.M. Papanikolas, J. R. Reynolds, and K. S. Schanze "Cyclometalated PlatinumContaining Diketopyrrolopyrrole Complexes and Polymers: Photophysics and Photovoltaic Applications" Chemistry of Materials, 29 (19), 8449-8461 (2017)

6. S. Guha and A. Laudari, "Polarization-induced transport in organic field-effect transistors: the role of ferroelectric dielectrics," Proc. SPIE 10365, Organic FieldEffect Transistors XVI, 103650I (2017) 
7. A. Pickett, A. Mohapatra, A. Laudari, S. Khanra, T. Ram, S. Patil, S. Guha, "Hybrid $\mathrm{ZnO}$-organic semiconductor interfaces in photodetectors: A comparison of two near-infrared donor-acceptor copolymers” Org. Electron. 45, 115-123(2017)

8. A. Laudari, S. Gangopadhyay, \& S. Guha, "Polarization-induced transport: A comparative study of ferroelectric and non-ferroelectric dielectric-gated organic field-effect transistors" MRS Advances, 1-6 (2017)

9. A. Laudari and S. Guha, "Bandlike transport in ferroelectric-based organic fieldeffect transistors" Phys. Rev. Applied 6, 044007(2016)

10. K. Gooden, A. Laudari, G. Knotts, and S. Guha, "Printed dielectric-based organic diodes and transistors" Flex. Print. Electron. 1, 015004 (2016)

11. A. Laudari and S. Guha, "Polarization-induced transport in ferroelectric-based organic field-effect transistors" J. Appl. Phys. 117, 105501 (2015)

12. T. Cipriano, G. Knotts, A. Laudari, R. Bianchi, W. A. Alves, and S. Guha, "Bioinspired peptide nanostructures for organic field-effect transistors" ACS Appl. Mater. Interfaces 6 (23) 21408 -21415 (2014) 


\section{Vita}

Amrit Prasad Laudari was born in a small village in Tanahun, Nepal. He received his BS and MS in Physics from the Tribhuvan University, Nepal in 2004 and 2007 respectively. After completion of his MS degree he was appointed as a lecturer in the Department of Physics at Kathmandu Model College, Nepal and served until Summer 2011. In the Fall of 2011, Amrit joined Missouri State University, Springfield as a graduate research assistant and got his MS degree in Materials Science under the supervision of Prof. Kartik Ghosh. In the Summer 2013, Amrit joined the University of Missouri, Columbia as a $\mathrm{PhD}$ research assistant in Physics under the supervision of Prof. Suchi Guha. His interests are focused on the thin film-based device fabrication and characterization.

In April of 2013, Amrit was married to Dipa Khaniya and they have a daughter born in May 19, 2017. 